\title{
EFFECT OF ATRIUM COMPARE TO COURTYARD ON ENERGY DEMAND AND DAYLIGHT IN ROW HOUSES IN TORONTO
}

\author{
by \\ Tamanna Kabir \\ Bachelor of Architecture \\ Bangladesh University of Engineering and Technology, 2008 \\ Bangladesh \\ MSc in Urban Management and Development, Specialization - Housing \\ Development Strategy \\ Erasmus University Rotterdam, 2011 \\ The Netherlands
}

\author{
A Major Research Project \\ presented to Ryerson University \\ in partial fulfillment of the \\ requirements for the degree of \\ Master of Building Science \\ in the Program of \\ Building Science
}

Toronto, Ontario, Canada, 2018

(C)Tamanna Kabir, 2018 


\section{Author's declaration}

I hereby declare that I am the sole author of this MRP. This is a true copy of the MRP, including any required final revisions.

I authorize Ryerson University to lend this MRP to other institutions or individuals for the purpose of scholarly research.

I further authorize Ryerson University to reproduce this MRP by photocopying or by other means, in total or in part, at the request of other institutions or individuals for the purpose of scholarly research.

I understand that my MRP may be made electronically available to the public. 


\section{Abstract}

Row houses in cold climates with increased shared wall for energy efficiency, suffer from darker interior spaces. Addition of courtyards can solve this problem to some extent, but courtyards are responsible for higher energy consumption during winter. This study investigates an alternative option, i.e. converting courtyard into atrium during winter for assessing its energy and daylight performance in row houses in Toronto. Results are determined by using Design Builder software. Research shows, during winter atrium options in row houses can reduce energy consumption compared to courtyard, but at the same time daylit floor area above target illuminance is also reduced. However, bigger courtyard having large window to wall ratio, clear glazing for courtyard windows and low e coated glazing for skylight can create a balance between increased energy consumption and decreased daylighting inside the house to maximize the benefits from converting courtyard into atrium during winter in Toronto row houses. 


\section{Acknowledgements}

First, I would like to thank Almighty Allah for His all the blessings in my life. I am really blessed to have the opportunity to study at Ryerson University in this unique course. Then my acknowledgement goes to my MRP supervisor Dr. Miljana Horvat for supporting me in my very crucial time. I am really grateful to her for her constant support, guidance, advice and encouragement. I am also very thankful to my second reader Dr. Umberto Berardi for his time to guide me to the right direction. I am also indebted to Dr. Russell Richman, Dr. Ramani Ramakrishnan for their support in this journey. It was not possible for me to finish this without unconditional support from my colleagues, Tonima Ferdous, Md Mostafizur Rahman and Jelena Madzarevic. Lastly, I want to thank my wonderful family for their care, endless support all the way. I am really proud and grateful to my 3 years old son, who sacrificed his mom's care greatly for my achievement. 


\section{Table of Contents}

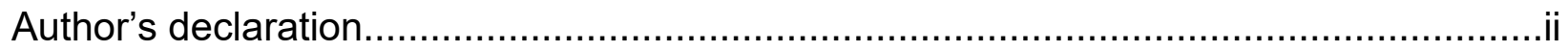

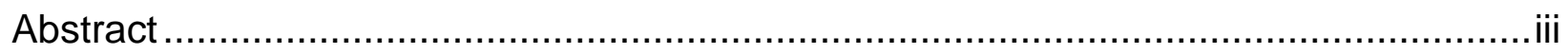

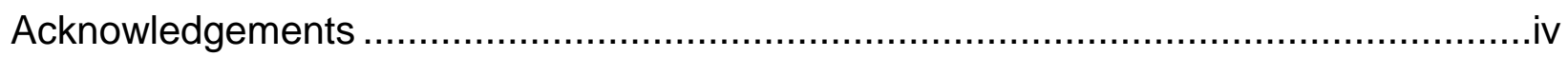

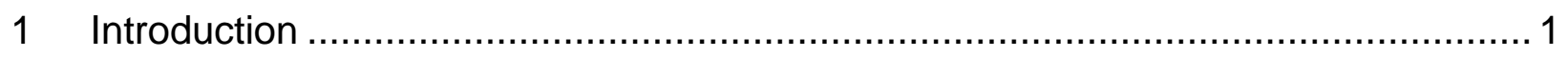

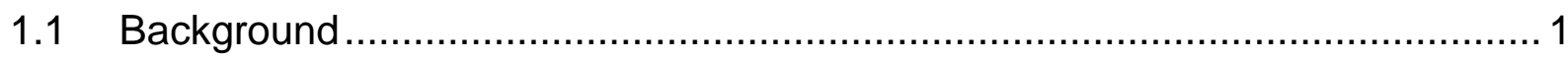

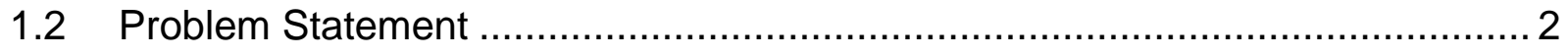

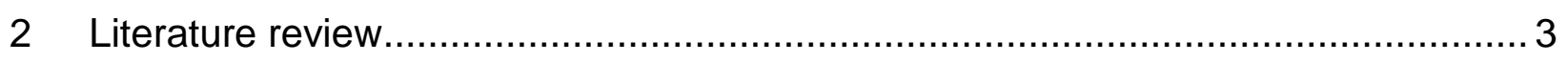

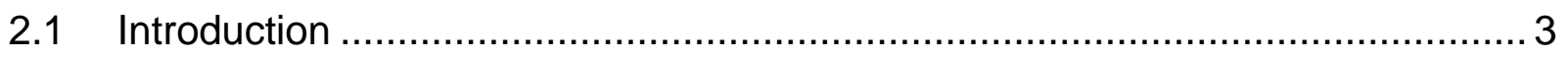

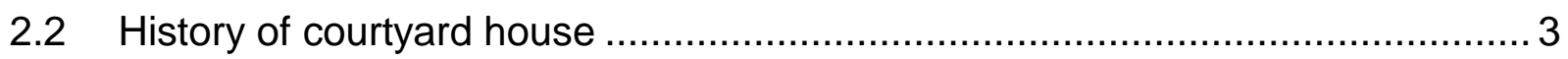

2.3 Use of courtyards in different climates ................................................... 5

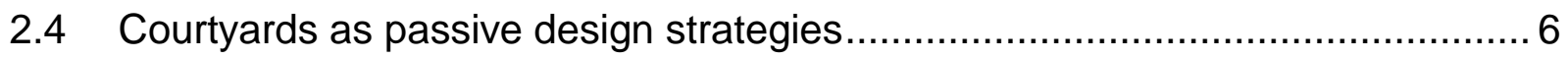

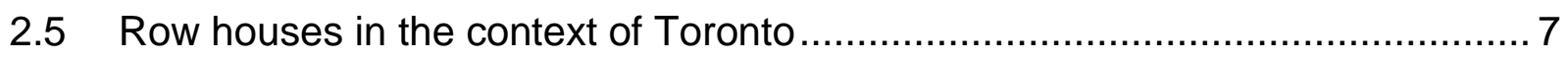

2.6 Factors influence the energy performance of row houses with courtyards......... 8

2.7 Studies on courtyard house in different climates for assessing their

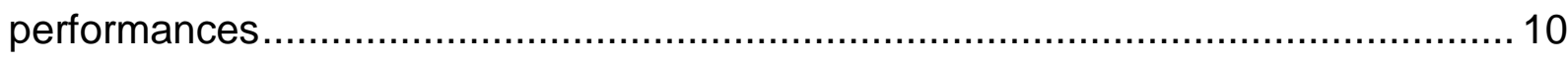

2.8 Energy and daylight performance of hybrid system i.e. open courtyard and

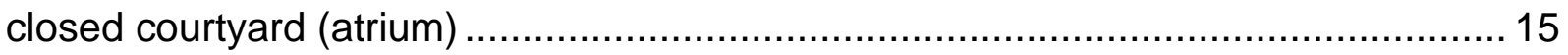

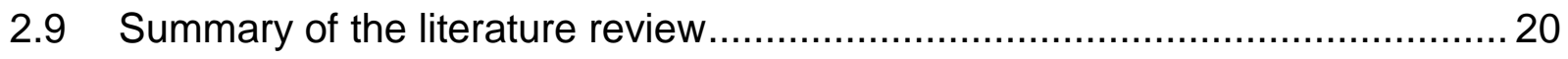

2.10 Objective and Research question ..................................................... 21

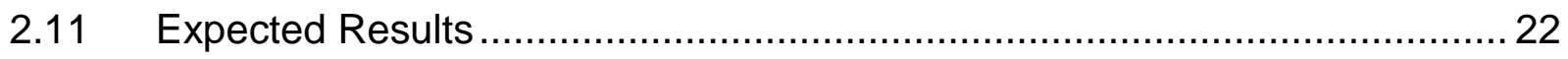


3 Methodology

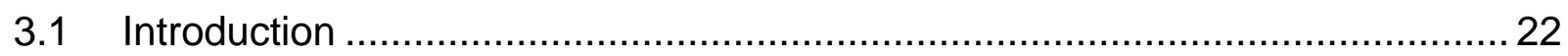

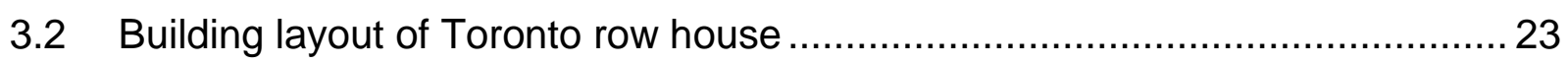

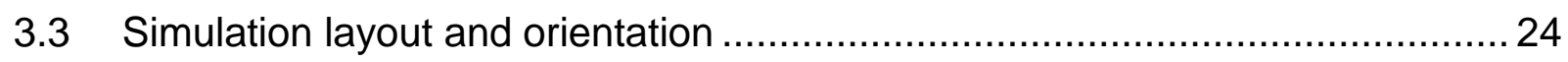

3.4 Courtyard size, orientation and glazing percentage …................................. 28

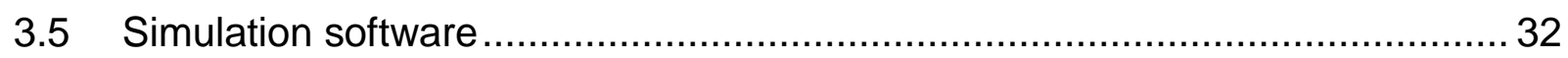

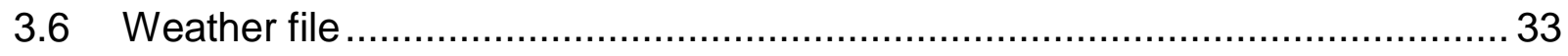

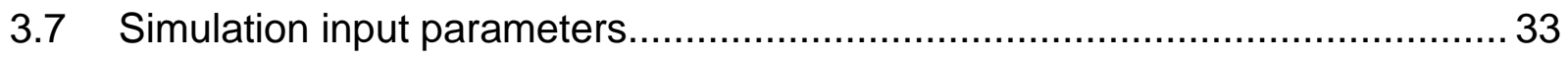

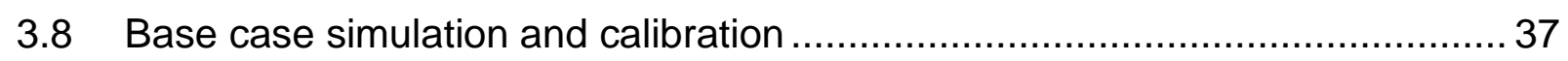

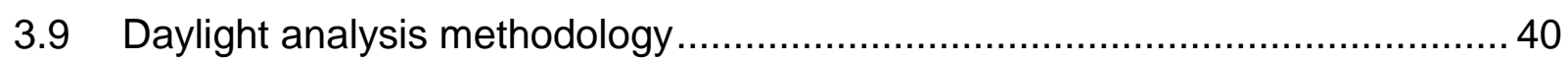

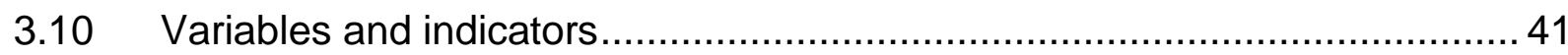

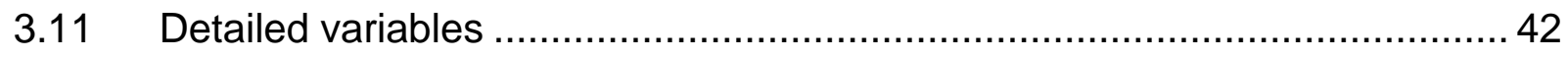

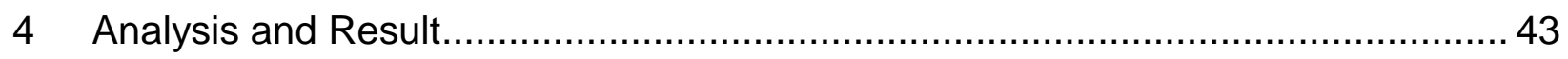

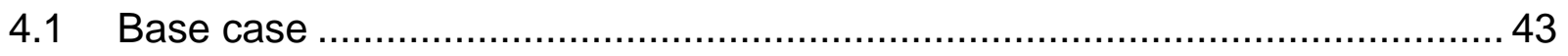

4.2 Energy performance of atrium house compared to courtyard house during winter

4.2.1 Reduction of energy consumption by atrium house during winter ............. 44

4.2.2 Energy performance of atrium house with different WWR …................... 47

4.2.3 Energy performance of atrium house with different orientation................. 48

4.2.4 Energy performance of atrium house with different glazing types ............ 49 
4.3 Daylight performance of atrium house

4.4 Combined calculation of energy consumption and illumination for optimization 55

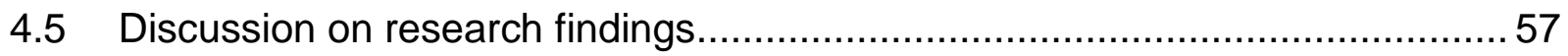

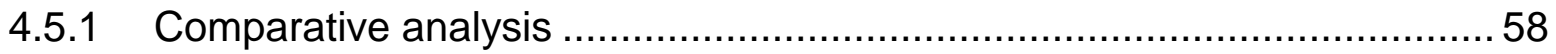

4.5.2 Connection between research result and existing knowledge ..................60

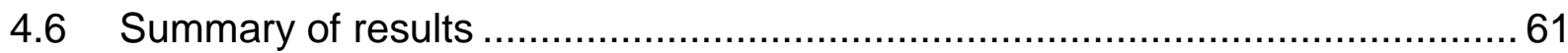

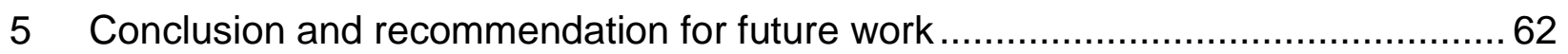

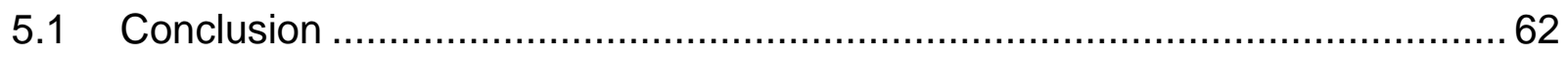

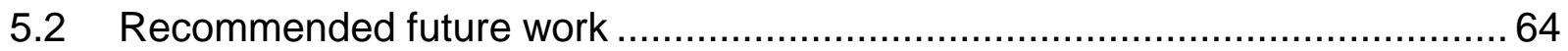

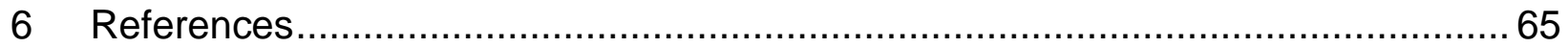

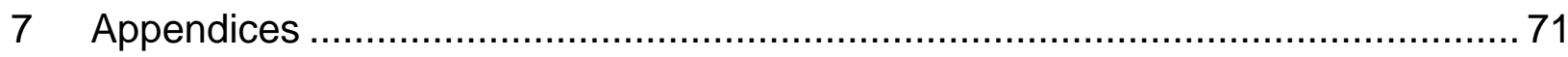

\section{List of Figures}

Figure 1 Courtyard and atrium in row house unit

Figure 2 Residence Andalous in Sousse, Tunisia. Serge Sautelli (Özkan, 2005) ..................................................

Figure 3 Middle age row house in Chester, UK. (Friedman, 2012) ...................................................................

Figure 4 Courtyards used to separate the kitchen from the living area and the room. Weekend house. Tokyo, 1998.

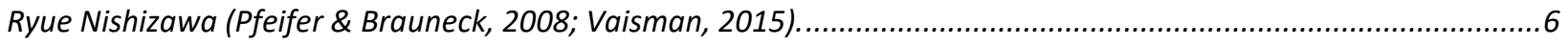

Figure 5 Row house buildings coverage averages $60 \%$ of the available land (De la Riva et al., 2000).......................7

Figure 6 Generic urban forms, based on Martin and March. From left to right: pavilions, slabs, terraces, terrace-

courts, pavilion-courts and courts (Martin \& March, 1972). 
Figure 7 Courtyard footprint configurations (Vaisman, 2015).

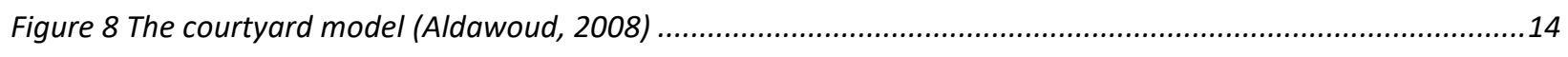

Figure 9 The research scenario (Taleghani et al., 2014) ..................................................................................

Figure 10 Two stage simulation process (Tabesh \& Sertyesilisik, 2016) .............................................................18

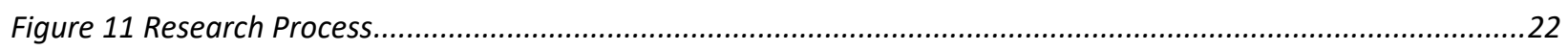

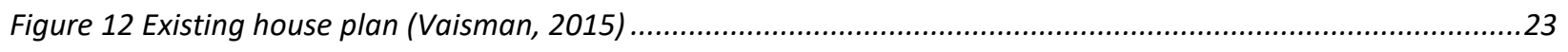

Figure 13 Interior and exterior of case study row house (Vaisman, 2015) .....................................................24

Figure 14 Elevations and section of the existing house. Units are in meters (Vaisman, 2015)...............................25

Figure 15 Simplified plans and elevations of the existing house. Units are in meters (Vaisman, 2015) ...................26

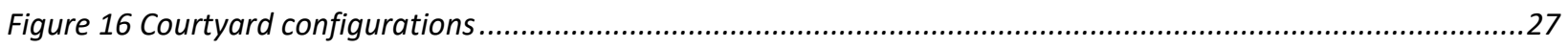

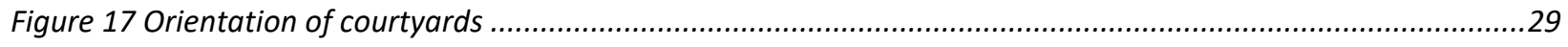

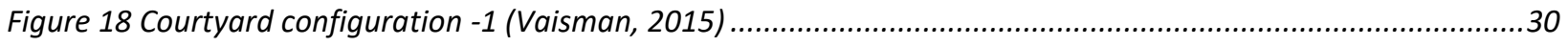

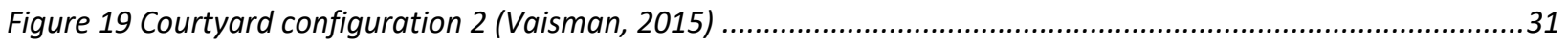

\section{List of Tables}

Table 1 Factors affecting the performance of row houses with courtyards ........................................................ 9

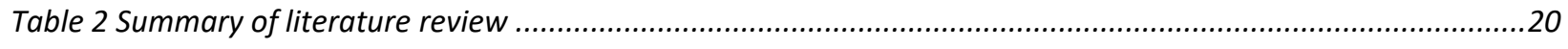

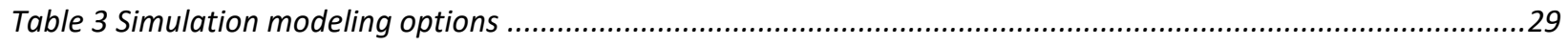

Table 4 SB-12 Package A requirements and specified material for the wall assembly........................................35

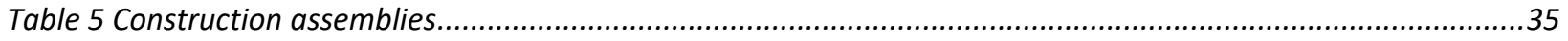

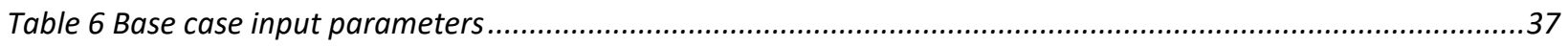

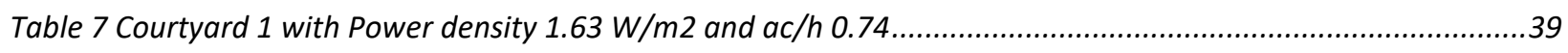

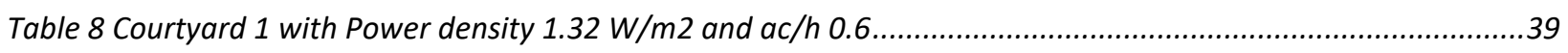

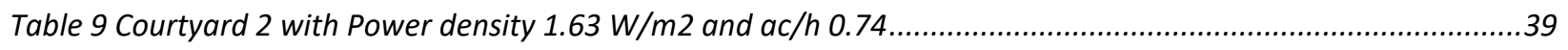

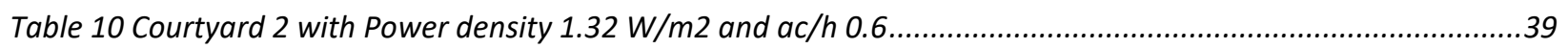

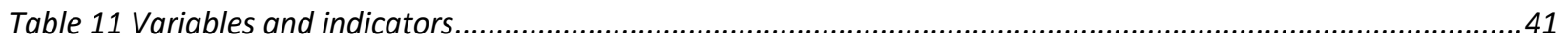


Table 12 Energy consumption of courtyard house for configuration -1.....

Table 13 Daylighting condition of courtyard house for configuration -1...

Table 14 Energy consumption of courtyard house for configuration -2. .43

Table 15 Daylighting condition of courtyard house for configuration -2.

Table 16 Reduction in energy consumption by atrium house compared to courtyard house for configuration -1 ......44

Table 17 Reduction in energy consumption by atrium house compared to courtyard house for configuration -2 ......44

Table 18 Improvement in energy consumption by atrium house compared to courtyard house .45

Table 19 Energy consumption of atrium house with different Window to Wall Ratio (WWR) 47

Table 20 Energy consumption of atrium house with different orientation 48

Table 21 Atrium's energy consumption for different glazing types 50

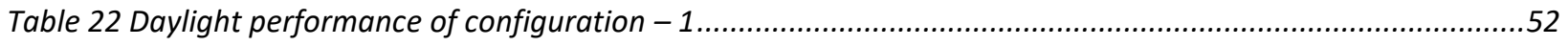

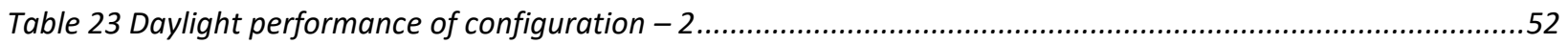

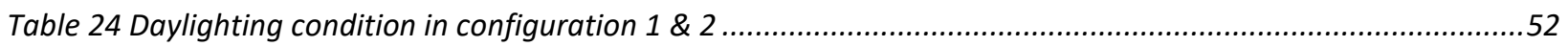

Table 25 Daylighting condition plotted for courtyard glazing Double clear, 6mm/13mm air and sky light Double clear, $6 \mathrm{~mm} / 13 \mathrm{~mm}$ air in configuration -1

Table 26 Total combination from different variables

Table 27 Areas of result's outcome. 56

Table 28 Table showing ten best optimized combinations......

Table 29 Table showing example comparison between best individual option and best optimum combination 59

\section{List of Appendices}

Appendix I Energy consumption by configuration -1, Option - 1 ..................................................................... 71

Appendix II Energy consumption by configuration -1 , Option -2 .................................................................

Appendix III Energy consumption by configuration -1, Option - 3 ................................................................

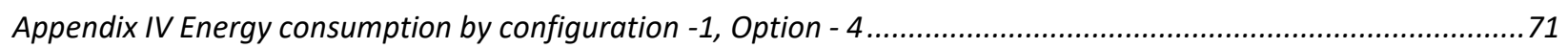

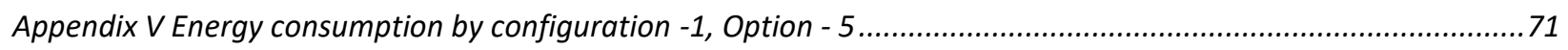


Appendix VI Energy consumption by configuration -1, Option - 6...............................................................

Appendix VII Energy consumption by configuration -1, Option - 7.............................................................. 72

Appendix VIII Energy consumption by configuration -1, Option - 8................................................................ 72

Appendix IX Energy consumption by configuration -2, Option - 1 ...............................................................

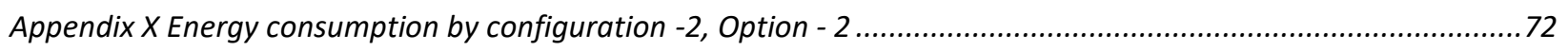

Appendix XI Energy consumption by configuration -2, Option - 3 ….............................................................73

Appendix XII Energy consumption by configuration -2, Option - 4 ....................................................................73

Appendix XIII Energy consumption by configuration -2, Option - 5 ................................................................

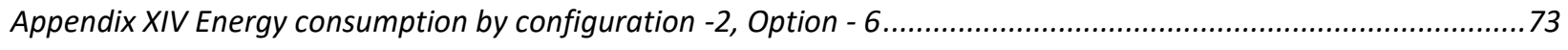

Appendix XV Energy consumption by configuration -2, Option - 7 ................................................................73

Appendix XVI Energy consumption by configuration -2, Option - 8............................................................... 74

Appendix XVII Daylighting condition of configuration -1, Option - 1................................................................. 74

Appendix XVIII Daylighting condition of configuration -1, Option - 2................................................................74

Appendix XIX Daylighting condition of configuration -1, Option - 3.............................................................. 74

Appendix XX Daylighting condition of configuration -1 , Option - 4 ................................................................ 74

Appendix XXI Daylighting condition of configuration -1, Option - 5................................................................. 75

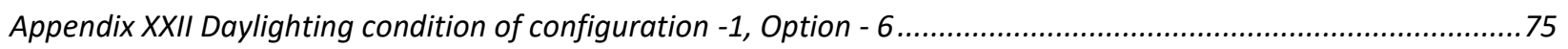

Appendix XXIII Daylighting condition of configuration -1, Option - 7 .............................................................. 75

Appendix XXIV Daylighting condition of configuration -1, Option - 8................................................................ 75

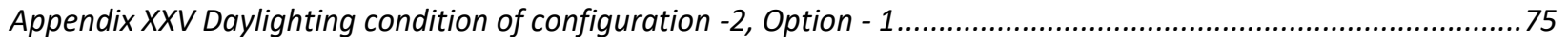

Appendix XXVI Daylighting condition of configuration -2, Option - 2................................................................... 76

Appendix XXVII Daylighting condition of configuration -2, Option - 3..............................................................76

Appendix XXVIII Daylighting condition of configuration -2, Option - 4...........................................................

Appendix XXIX Daylighting condition of configuration -2, Option - 5................................................................ 76

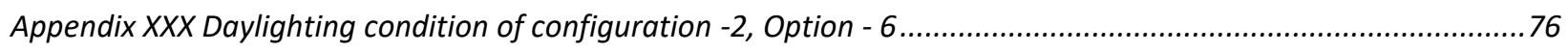

Appendix XXXI Daylighting condition of configuration -2, Option - 7.............................................................77

Appendix XXXII Daylighting condition of configuration -2, Option - 8...................................................................77 
Appendix XXXIII Improvement in energy consumption by atrium house compare to courtyard house ......................77 Appendix XXXIV Energy consumption of atrium house with different WWR ......................................................79 Appendix XXXV Energy consumption of atrium house with different orientation ..................................................8 82 Appendix XXXVI Energy consumption of atrium house for different glazing types ...............................................8 Appendix XXXVII Comparison of daylighting condition between courtyard house and atrium house........................94 Appendix XXXVIII Daylighting condition plotted for courtyard glazing Double LoE (e2=.1) Clr 6mm/13mm Air and sky

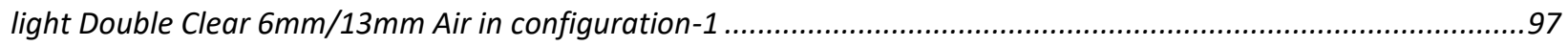

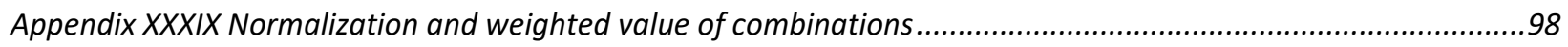

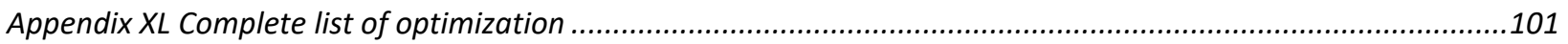




\section{Introduction}

\subsection{Background}

Row house refers to the higher construction density houses in the realm of architecture and urban planning (De la Riva, Gagnon, \& Affleck, 2000). However, in building science this archetype manifests the lower energy consumption by less exposed surfaces and shared wall with adjacent units. This type of housing can reduce up to $68 \%$ of energy depending on its locations and compared to detached houses (Friedman, 2012). Row houses are originated in Europe in $16^{\text {th }}$ century, brought to North America by British migration and became common typology of dwelling in some North American cities including Toronto (Ward, 1999). There are about 179,100 row houses in Toronto and it comprises $9 \%$ of total housing stock of the city (CMHC, 2014). Although this percentage is not very high, with the increasing property price and lack of available land for housing construction, row house can be a suitable option to own a house with achieving energy efficiency and urban density (Vaisman, 2015). Nevertheless, one of the major problems of row house is associated with less exposed exterior wall for windows. So, the performance of row house for natural lighting and ventilation is not convincing. Center of the row house always gets lower level of natural lights and less hours of illuminance. By detaching the back end of the side wall from the adjacent houses some older row houses were able to generate a bay of light and air within the middle of the row houses. But this configuration is almost absent in new and retrofitted constructions (Vaisman, 2015). To address this issue, different transitional spaces have been identified to get natural light and air. These are varied ranges of spaces including courtyard, atrium, 
pavilion, slab etc. (Taleghani, Tenpierik, \& Dobbelsteen, 2014). In world architecture this type of solutions has been using for 5000 years (Fathy, 1986; Oliver, 2003).

Though history of the row houses is not new in Toronto, but the courtyard or atrium in row house is not a very common practice. The centers of the most of the newly built or retrofitted row houses are dark and they only get good natural light close to front and back windows (Vaisman, 2015).

\subsection{Problem Statement}

German Vaisman (2015) conducted a research at Ryerson University in Toronto to show the impact of courtyard in row houses in Toronto in order to improve natural light and hours of illumination. He also investigated the energy demand in that type of settings. His research result showed that incorporation of courtyard in row houses can improve natural lighting condition in the middle of the row houses. Also, hours of illuminance can be increased by $9 \%$ to $20 \%$. Internal courtyard provides better response when the light is more needed. In winter months there is an increase of over two hours of illuminance compared to a row house without courtyard. But the row houses with the internal courtyard can use $13 \%$ to $36 \%$ more energy in winter than a row house without courtyard (Vaisman, 2015).

Vaisman's research revealed that while internal courtyard offers a unique opportunity to maximize the time with natural light inside the row house in a colder climatic location like Toronto, but at the same time this type of internal courtyard has a significant influence on energy load with increased exterior wall and glazing. 

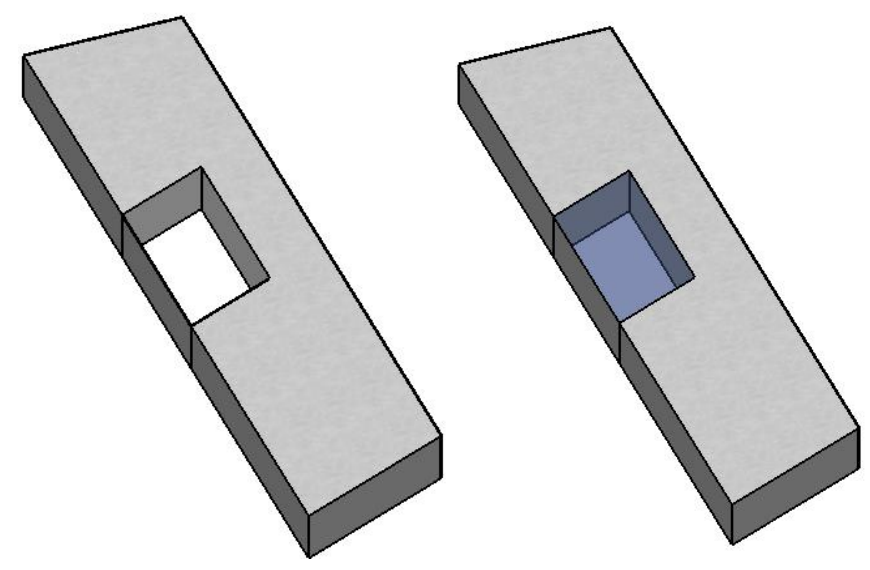

Figure 1 Courtyard and atrium in row house unit

So, it is important to investigate that how this increased energy load can be reduced in row houses with courtyard during colder months in semi continental climate of Toronto.

\section{Literature review}

\subsection{Introduction}

Existing knowledge in energy and daylight performance of buildings with open courtyard will help to develop the base case of this research. Study of hybrid system (i.e. open courtyard in summer and closed courtyard in winter) in row houses and other functional type of buildings in different climate will help to understand which variables are critical for hybrid system and how-to setup model for simulation for this research - while the researcher intends to identify the impact of hybrid system in row houses in Toronto in terms of energy consumption and daylighting performance.

\subsection{History of courtyard house}

The history of the courtyard house was started at the beginning of the human settlement when protection from animals and from human invasions were of main concern. In 
Neolithic age courtyards were used as a protective configuration (Özkan, 2005). In different cultures courtyards were adopted in houses for different purposes and later they were flourished accordingly (Petruccioli, 2006). Petruccioli, A. (2006) also showed in his analysis that how courtyard was evolved from an enclosed wall to additional cell around it (Petruccioli, 2006). In Islamic architecture, it is a cultural addition to housing for gender separation (Özkan, 2005).

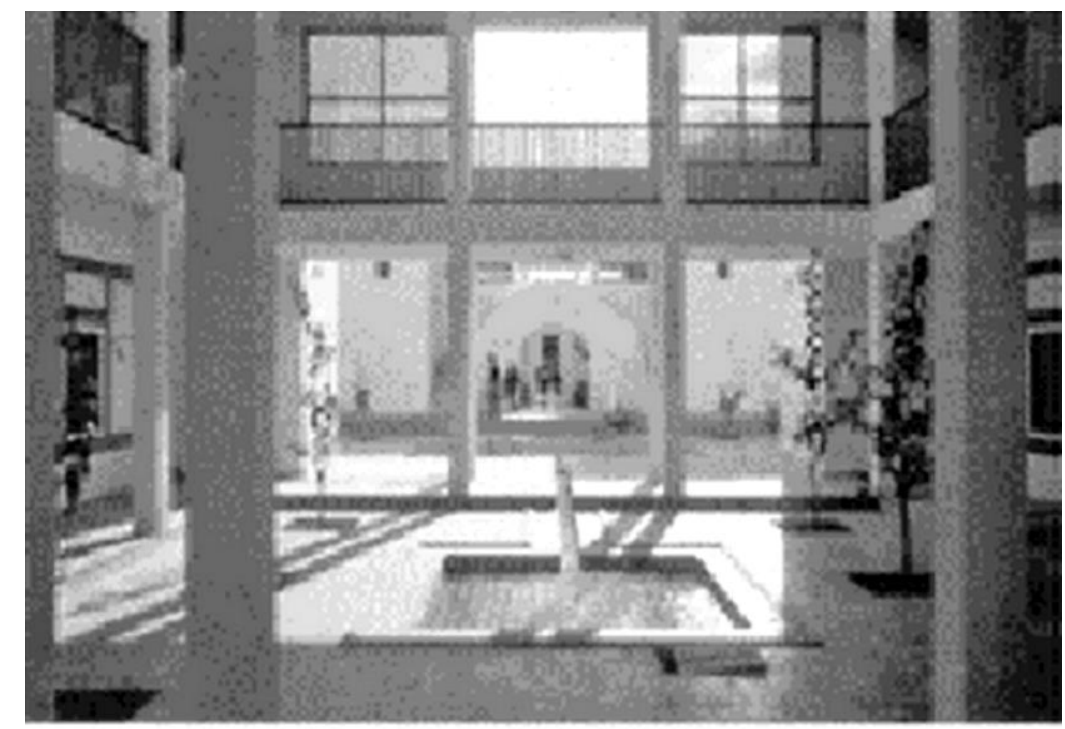

Figure 2 Residence Andalous in Sousse, Tunisia. Serge Sautelli (Özkan, 2005)

In some cultures, courtyards were adopted as service separators in houses. In Roman row houses courtyards were used for bringing light and fresh air in the buildings (Friedman, 2012). 


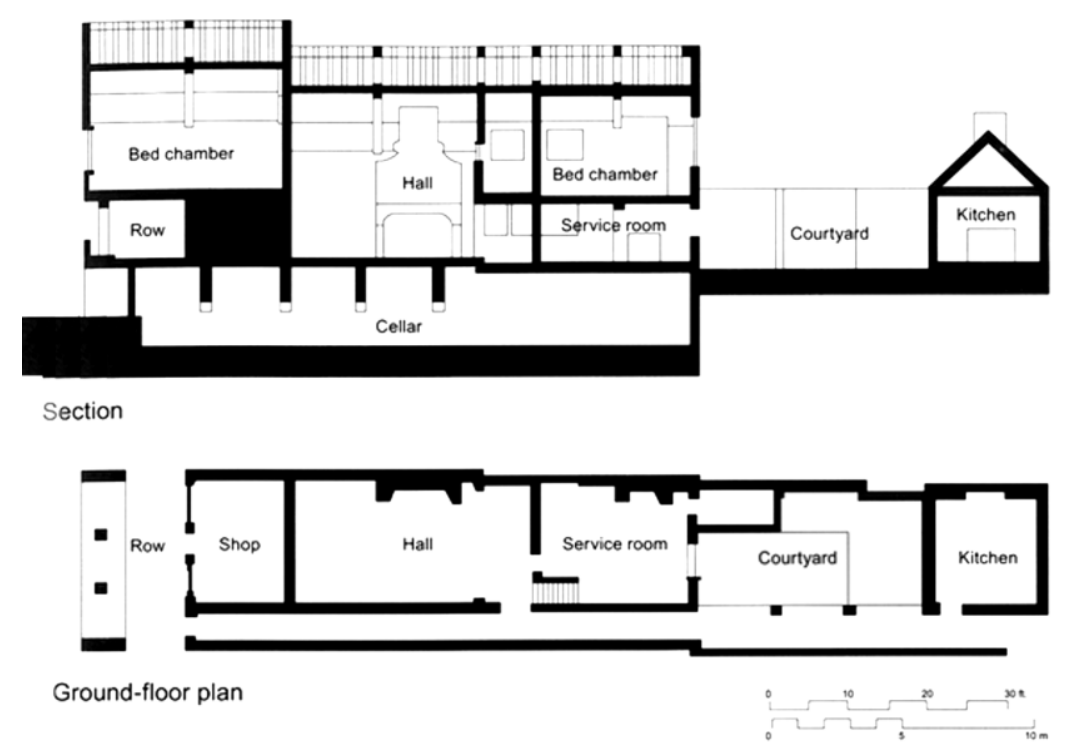

Figure 3 Middle age row house in Chester, UK. (Friedman, 2012)

\subsection{Use of courtyards in different climates}

Use of courtyard for climatic reason has been in place for a long time in hot climates in order to create a cooler microclimate. There are significant number of studies are found for courtyard type housing in hot climates. Some examples of courtyard houses are also found in cold climates' architecture. This typology was occasionally adopted in continental Europe and Scandinavian architecture. But not so many literatures are found on this topic (Culjat, 1988; Vaisman, 2015). In cold climates, the functions of courtyards are mainly of creating microclimate with increased air temperature, protection from cold wind and for extending the 'outdoor season' by accommodating outdoor activities (Liu, 1988; Raydan, Ratti, \& Steemers, 2003; Vaisman, 2015). Research showed that in Norway people usually have 64 to 133 comfortable days a year in outdoor temperatures and up to 42 more days can be added to that including courtyard into the house (Vaisman, 2015). 


\subsection{Courtyards as passive design strategies}

In current practices, courtyard houses are mainly used for passive design strategies.

Studies are found on courtyards' lot sizes, sun angles, code restrictions etc. to get maximum benefit from courtyard houses ensuring comfortable indoor and usable outdoor spaces with privacy (Pfeifer \& Brauneck, 2008; Vaisman, 2015).
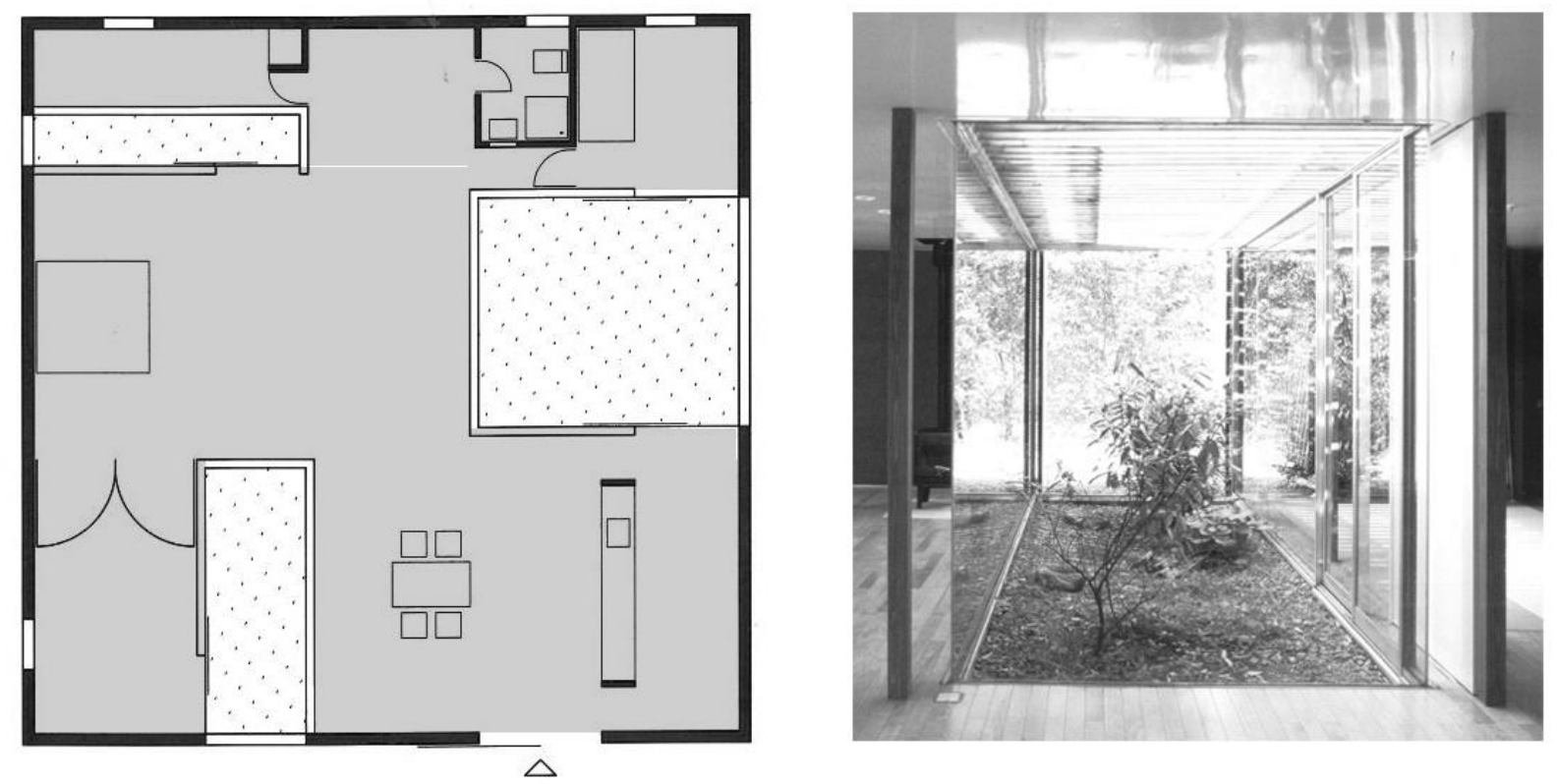

Figure 4 Courtyards used to separate the kitchen from the living area and the room. Weekend house. Tokyo, 1998. Ryue Nishizawa (Pfeifer \& Brauneck, 2008; Vaisman, 2015).

Daylight control, ventilation, orientation, optimal shape, size, energy efficiency, ecological design etc. became more important while designing courtyard houses (Friedman, 2012; Guzowski, 2010; Snøhetta (Architectural firm), 2018). Courtyards are found in different types of functional buildings ranging from commercial to residential. In the row houses where density policies are trying to achieve in residential scale, internal courtyards offer access to exterior spaces with bringing the daylight in the middle of the units (Vaisman, 2015). 


\subsection{Row houses in the context of Toronto}

Toronto row houses with courtyards it is not very well studied topic till now (Vaisman, 2015). The potential of row houses with courtyards is needed to be explored for the context of Toronto to get more benefits from this housing typology. In legislative documents definition of row house is clearly outlined, as in one form Canada Mortgage and Housing Corporation (CMHC, 2012), which defines "one of three or more dwellings joined side by side (or occasionally side to back), such as a townhouse or garden home, but not having any other dwellings either above or below. Townhouses attached to a high-rise building are also classified as row houses." (CMHC, 2014). This housing typology was brought in North America by British migration (Ward, 1999). In a highly dense urban fabric row houses offer a high construction density with an average of $60 \%$ available land coverage (De la Riva et al., 2000).

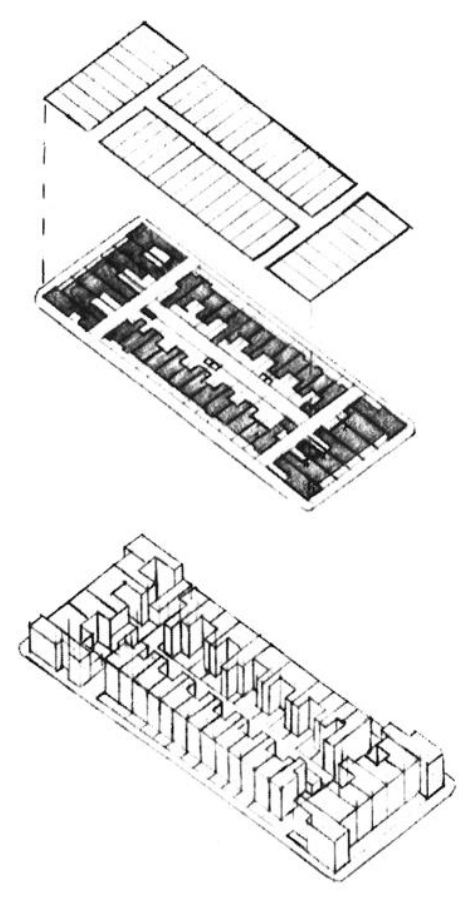

Figure 5 Row house buildings coverage averages 60\% of the available land (De la Riva et al., 2000) 


\subsection{Factors influence the energy performance of row houses with courtyards}

Row house with courtyard is addressed by two issues in terms of energy consumption.

Energy consumption in row house is lower because of less exposed exterior surfaces.

But inclusion of courtyard in row house increases the exposed surfaces, thus energy consumption is also increased in cold climate (Vaisman, 2015). From literature, it is evident that the following factors are directly related to the performance of the row houses with courtyards: 
Table 1 Factors affecting the performance of row houses with courtyards

\begin{tabular}{|c|c|c|}
\hline $\begin{array}{l}\text { Factors of } \\
\text { courtyards }\end{array}$ & $\begin{array}{l}\text { Optimum forms/configuration for preferable energy } \\
\text { performance }\end{array}$ & Reason \\
\hline Shape & Rectangular shape than others shapes with many corners. & $\begin{array}{l}\text { Floor to surface ratios: } \\
\text { the higher the ratio } \\
\text { the lower the energy } \\
\text { consumption. } \\
\text { (Friedman, 2012) }\end{array}$ \\
\hline Length & $\begin{array}{l}9 \mathrm{~m}(30 \mathrm{ft}) \text { maximum length of house oriented to North-South } \\
\text { and between } 12 \mathrm{~m}-14 \mathrm{~m}(40-46 \mathrm{ft}) \text { oriented to East West } \\
\text { orientation for entering natural light inside the core of the } \\
\text { house. }\end{array}$ & $\begin{array}{l}\text { Daylight entering the } \\
\text { house according to the } \\
\text { orientation (Friedman, } \\
\text { 2012) }\end{array}$ \\
\hline $\begin{array}{l}\text { Height to } \\
\text { Width ratio }\end{array}$ & $\begin{array}{l}\text { Average height to width ratio } 0.6 \text { for concentrating sun in } \\
\text { Scandinavian country and } \\
1.3 \text { in hot climate. }\end{array}$ & $\begin{array}{l}\text { The proportion } \\
\text { between height and } \\
\text { width are critical for } \\
\text { designing the } \\
\text { courtyard that collect } \\
\text { sun or courtyard to } \\
\text { protect from direct } \\
\text { sun (Muhaisen, 2006 } \\
\text { in Vaisman, 2015) }\end{array}$ \\
\hline Story & $\begin{array}{l}\text { Good performance can be obtained from one story for } \\
\text { summer and winter in cold climate. }\end{array}$ & $\begin{array}{l}\text { Increase the surface } \\
\text { area ratio reached by } \\
\text { sun (Muhaisen, 2006) }\end{array}$ \\
\hline Orientation & $\begin{array}{l}\text { In most of the locations rectangular courtyard around the } \\
\text { North South axis exposed more to sun light than East-West } \\
\text { axis. }\end{array}$ & $\begin{array}{l}\text { Orientation and } \\
\text { location together } \\
\text { determine how much } \\
\text { internal courtyard's } \\
\text { walls will be exposed } \\
\text { to sun light }\end{array}$ \\
\hline
\end{tabular}




\subsection{Studies on courtyard house in different climates for assessing their performances}

In hot climate, to reduce energy consumption and to bring natural light and air inside the house, traditional spaces like courtyard have been used for long time (Fathy, 1986; Oliver, 2003). In this type of transitional space without mechanical control system, indoor and outdoor climate is moderated, where the occupants may experience dynamic effects of outdoor climate changes to a certain extent (Taleghani et al., 2014). Martin and March (1972) examined six simplified archetypal forms to analyse and compare the archetypes in terms of built potential and daylighting criteria. These examined forms are: pavilions, slabs, terraces, terrace-courts, pavilion-courts and courts. In courtyard house, open areas are 'dispersed' instead of 'clot' together. When the courtyard house was compared with pavilion type house, it was found that courtyard form can place same amount of floor space with same site and condition of building depth, but it requires one third height than requires by pavilion form. That means in exactly same setting and with same building properties, courtyard house can provide same functional space with less height compared to pavilion house (Martin \& March, 1972).
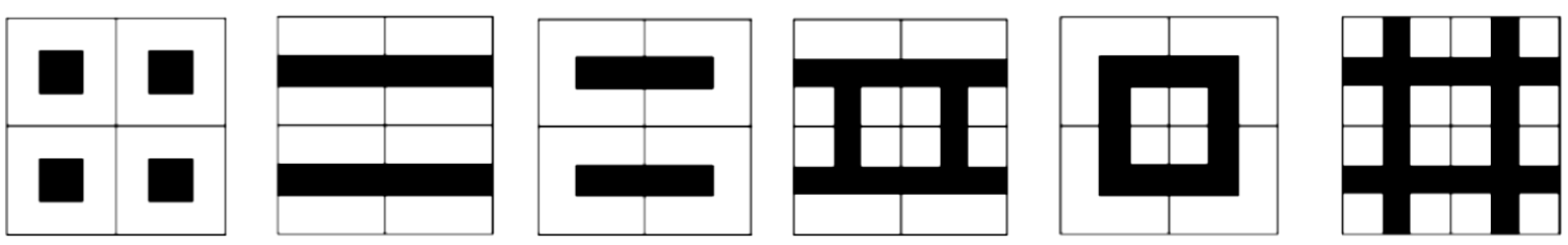

Figure 6 Generic urban forms, based on Martin and March. From left to right: pavilions, slabs, terraces, terracecourts, pavilion-courts and courts (Martin \& March, 1972). 
Steemers at al (1997) employed Martin and March (1972)'s generic urban forms to address the relationship between urban microclimate and form identifying the key environmental characteristics for the location of London $\left(51^{0} \mathrm{~N}\right)$. Comparing daylighting, solar radiation and built potential they also found that, the performance of courtyard form is better than other five generic urban forms in terms of their built potential and daylighting criteria (Martin \& March, 1972; Steemers et al., 1997). Later Ratti et al (2003) reassessed the Martin and March (1972)'s findings using innovative computer analysis technique for hot arid climate. They found that, if the forms are interpreted in environmental terms without sacrificing floor space for a given plot of land, courtyard form is best. Courtyard can create a microclimatic context, where low energy strategies through the limitations of air conditioning loads are possible. Changes in courtyard proportions can also change its energy and daylighting performance. In cold climates, under certain geometrical conditions (surface to volume ratio 0.58 ), large courtyards are environmentally adequate. They can act as sun concentrators and against wind they can retain their sheltering effect (Ratti, Raydan, \& Steemers, 2003).

German Vaisman (2015) investigated energy and daylight performance of row houses with open courtyard in Toronto. The study also gives indication for optimal configuration of courtyard in row houses. He used an existing typical row house for the study which is located in Toronto. In that study he designed four (4) courtyard footprints and each courtyard had four (4) different window to wall ratios. Shapes of the courtyards are,

Courtyard - 1, 1:1.5 - Rectangular,

Courtyard -2, 1:1 - Square, 
Courtyard - 3, 1:2-Rectangular,

Courtyard - 4, 2:1 - Rectangular

Window to wall ratios can be grouped into following rages,

A - $73 \%$ to $100 \%$,

B - $55 \%$ to $65 \%$,

C - $36 \%$ to $42 \%$,

D - $20 \%$ to $34 \%$

Courtyard \#1

Rectangular

$1: 1.5$

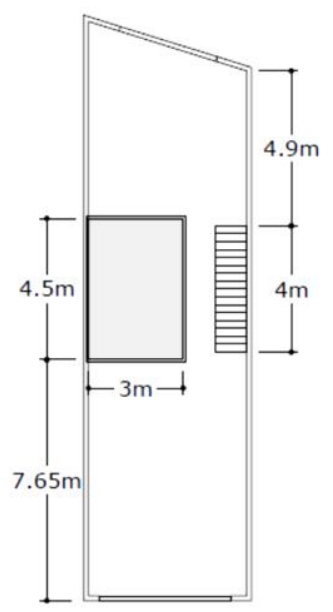

Courtyard \#2

Squared

$1: 1$

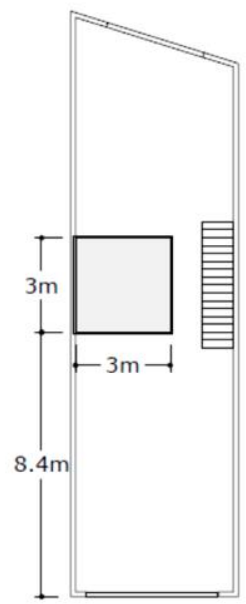

Courtyard \#3

Rectangular

Vertical

$1: 2$

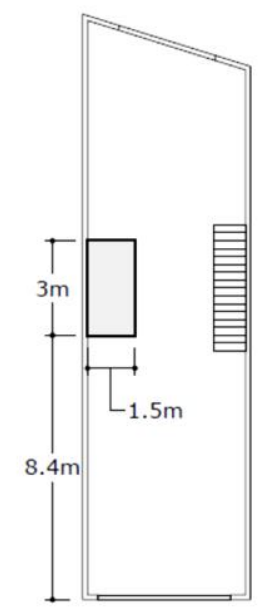

Courtyard \#4

Rectangular

Horizontal

2:1

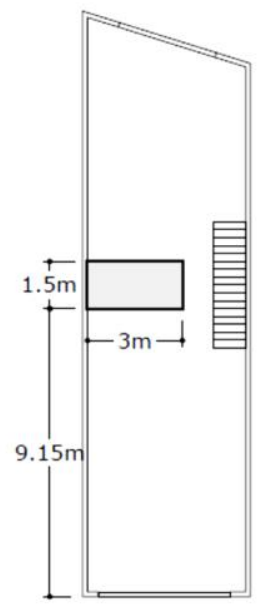

Figure 7 Courtyard footprint configurations (Vaisman, 2015)

All those configurations were also tested for 4 orientation, i.e. North, East, South, West (Vaisman, 2015). 
His study revealed that introduction of courtyard in the row house improves daylighting condition inside the house. He simulated 64 options and among them 56 options were showed improvement in hours of illuminance. This improvement is found in the middle of the house, specially in winter months when days are shorter. This increase ranges from $9 \%$ (for courtyard -3 with $55 \%$ glazing) to $20 \%$ (for courtyard- 1 with $57 \%$ glazing). If the energy saving lights like CFLs or LEDs are used in the house, with the increase of daylight inside the house, small energy saving is found from the use of artificial lights. This saving ranges from $1.14 \%$ to $1.42 \%$. The result of the study shows that, window to wall ratio does not have major influence on hours of illuminance, but larger courtyards influence the hours of illumination. The study also shows that while courtyard Improves daylighting condition inside the house, at the same time courtyards are responsible for $13 \%$ to $36 \%$ more energy use with high window to wall ratio (WWR) and with increased exterior wall. Finally, the study suggested that larger courtyards with $20 \%$ to $40 \%$ WWR is an optimal solution for getting more hours of illuminance with less increase in energy demand (Vaisman, 2015).

Aldawoud, A. (2008) compared thermal performance of courtyard in four (4) climatic regions. He studied four climatic regions of United States: Phoenix, Arizona for hot-dry climate, Miami, Florida for hot-humid climate, Chicago, Illinois for temperate climate, Minneapolis, Minnesota for cold climate. He used square courtyard for the study. Other than four climatic regions, the variables he used were height, glazing type and glazing percentage. For height of the courtyard this study considered 1 to10 story height, 4 glazing types - single clear glass, double clear glass, triple glass and double low e glass were used. Two glazing percentages: $30 \%$ and $67 \%$ were used (Aldawoud, 2008). 

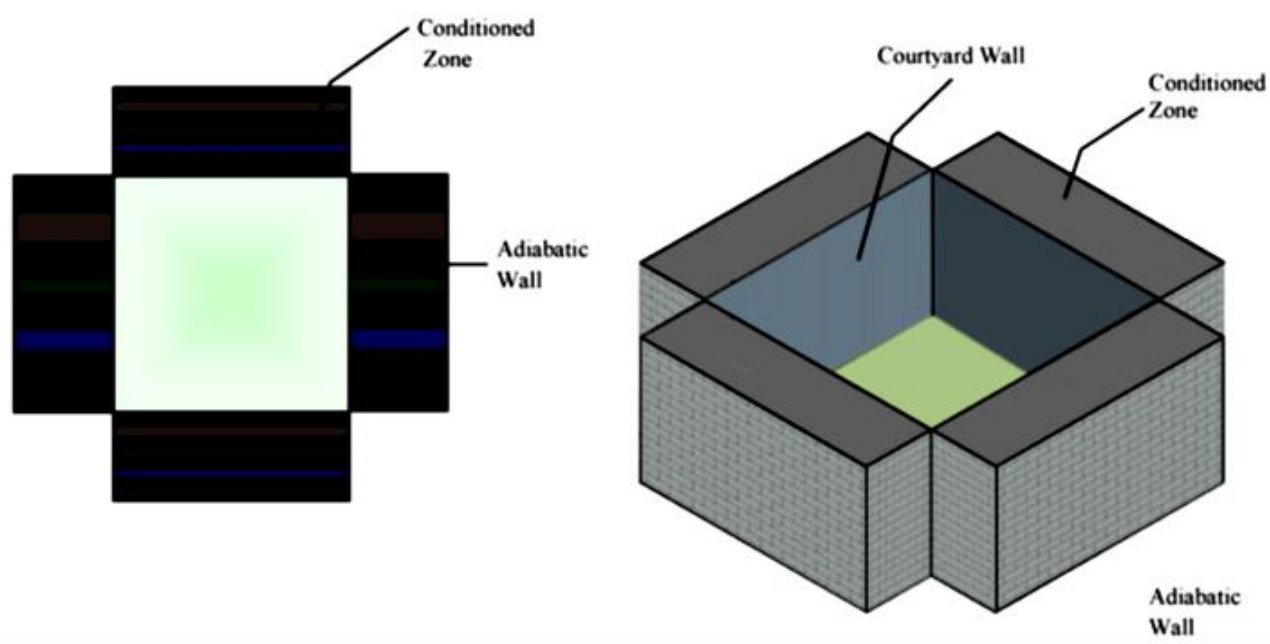

Figure 8 The courtyard model (Aldawoud, 2008)

The study confirms that the buildings are in hot humid climate can get maximum benefit in terms of energy savings from this type of open courtyard. In this case maximum benefits can be achieved from triple clear glazing and minimum benefit can be achieved from single clear glazing (energy consumption is $12 \%$ less with triple clear glazing than with single clear glazing). After that hot-dry climate can save more energy with open courtyard. Temperate and cold climate hold 3rd and 4th position in terms of energy use reduction. That means among these 4 climates, thermal performance of courtyard house in cold climate is worse. To understand this statement one findings from the study can be explained, a courtyard having triple clear glass can reduce energy consumption by $45 \%$ in hot humid climate, by $29 \%$ in hot-dry climate, by $20 \%$ in temperate climate compare to cold climate. From the different variable heights, it was found that for all climate single story courtyard works better. With the increase of the height of the courtyard, thermal performance of the courtyard house deteriorates significantly. Among the all glazing types performance of triple clear glass was best for all climatic condition, but double low-e glass performs better than other 2 options in all 
climatic conditions. Percentage of glazing (WWR) is another important parameter that significantly influence thermal performance of the open courtyard house. This study finds that for cold climate, courtyard with $30 \%$ glazing percentage and triple clear glass can be optimum solution (Aldawoud, 2008).

Another study developed by Al-Hemiddi and Megren Al-Saud (2001) shows that, cross ventilation in courtyard significantly improve interior thermal comfort and reduce cooling load (Al-Hemiddi \& Megren Al-Saud, 2001). Orientation also influences the courtyard's performance, like in hot arid climate East-West oriented courtyard creates more discomfort than North-South oriented courtyard (Meir, Pearlmutter, \& Etzion, 1995). Another benefits of courtyard in snow climate is that, it protects buildings from cold winds (Mänty \& Pressman, 1988; Vaisman, 2015).

\subsection{Energy and daylight performance of hybrid system i.e. open courtyard and closed courtyard (atrium)}

Hybrid system of courtyard refers to that option of a courtyard building that can be kept open to sky and can be covered with glazing (atrium) when it is necessary. But there is a need to have a flexible open space inside the building to accommodate these two options (Taleghani et al., 2014). However, the use of both open courtyard and atrium in a same building is not a common practice. Usually in buildings, either open courtyards or atriums are used. Traditionally in hot-dry and hot-humid climates, courtyards are used to create cooler microclimate and to reduce humidity by shading effect of courtyard (AI-Hemiddi \& Megren Al-Saud, 2001; Haw, Saadatian, Sulaiman, Mat, \& Sopian, 2012; Rajapaksha, Nagai, \& Okumiya, 2003). In colder climate atriums are 
intentionally used to reduce heat loss and capture solar energy (Ayoob \& Izard, 1994; Edwards, Sibley, Hakmi, \& Land, 2006; Laouadi, Atif, \& Galasiu, 2003). There are similarities in the use of courtyard and atrium in buildings, for example, both are used for bringing daylight, providing ventilation and creating buffer zone between indoor and outdoor environment. But most of the commercial and institutional courtyard or atrium buildings are fully depend on mechanical systems and do not take the advantage of these spaces as a tool to minimize energy consumptions (Tabesh \& Sertyesilisik, 2016). Shapes, no of story, glazing percentage, glazing type of courtyards and atriums are needed to be considered in design phase for proper functioning of these two transitional spaces (Aldawoud, 2013). These types of spaces in building enhance daylight condition in interior and consequently minimize space conditioning and lighting loads (Aldawoud \& Clark, 2008). The design of atrium which fails to fit in a specific context can lead to unintended result and increase in energy consumption (Aldawoud, 2013). Design of atrium with feasible glazing area, glazing type and height for particular location can create a comfortable, visually pleasant environment along with capture of solar energy to reduce heating load (Mills, 1991) in (Tabesh \& Sertyesilisik, 2016).

Taleghani et al (2014) investigated energy performance and thermal comfort of hybrid system in terraced houses (row houses) in the temperate climate of the Netherlands. Their simulation also predicted the energy consumption scenario in 2050 in the Netherlands. They considered typical Dutch mid-terraced dwelling having small courtyard. Window to wall ratio was taken around $30 \%$ which is very common in the Netherlands. Due to high latitude $\left(52^{\circ} \mathrm{N}\right)$ and low sun angle during the winter, usually most Dutch houses uses larger window to achieve maximum daylight access (Taleghani 
et al., 2014). For simulation researcher also considered double glazing with an air cavity of $13 \mathrm{~mm}$ in between 2 layers.

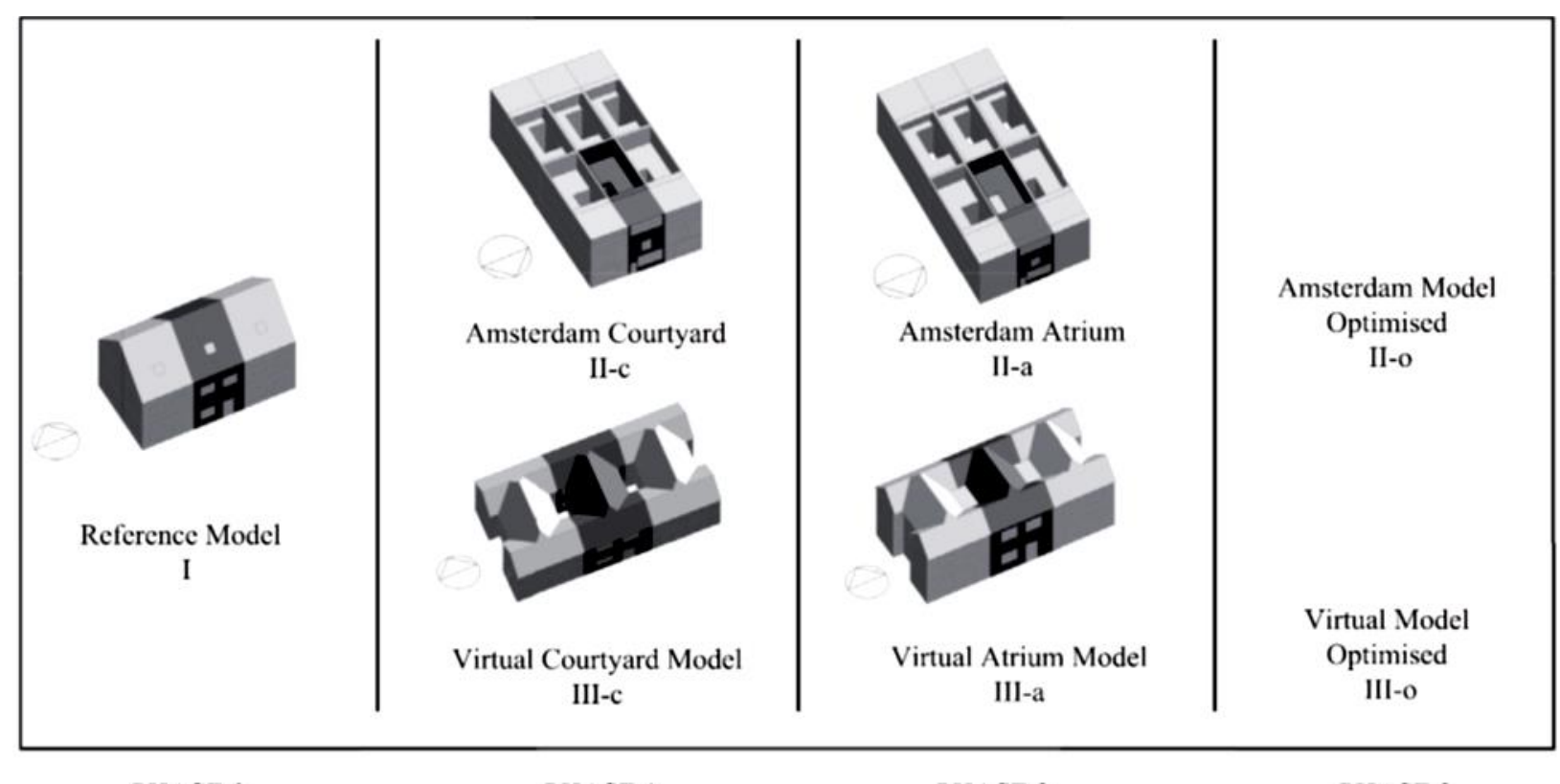

PHASE 0

PHASE 1

PHASE 2

PHASE 3

Figure 9 The research scenario (Taleghani et al., 2014)

The result of that research showed that courtyard reduces indoor operative temperature in winter (which reduces energy consumption), increases comfort hours. Courtyards do not relate to energy consumption in summer since the Dutch houses are in free running mode in summer, that means they do not use any additional mechanical equipment for cooling in summer. Courtyard houses also showed increased energy consumption during winter due to higher exposure to outdoor environment. However, when the dwelling tested with glazed roof over courtyard instead of open courtyard, the result showed that in winter days atrium reduces heat loss, thus deceases the heating load, but in summer time it increases thermal discomfort hours. The average winter monthly energy consumption difference between courtyard house and atrium house is found 2.3 $\mathrm{kWh} / \mathrm{m}^{2}$. Their optimization showed that courtyard need to be open at least for 4 months, 
i.e. May to August. In the period of November to April courtyard should be covered with glass. In September to October, courtyards or atrium modes perform equally well due to moderate climatic condition (Taleghani et al., 2014).

Tabesh et al (2016) researched on integrated usage of a courtyard and atrium in 3 different climatic conditions of Turkey. They used offices and retails for their study. They chose Istanbul for mild/humid climate, Erzurum for cold climate and Diyarbakir for hotdry climate (Tabesh \& Sertyesilisik, 2016). From their literature search they revealed that square courtyard is optimal in terms of energy demand while rectangular courtyard can affect shading and exposure condition (Muhaisen \& Gadi, 2006). So, they used square courtyard for all climates and in the same way they selected three story courtyards in hot-humid climate, 2 story in hot-dry and temperate climate and one story for cold climate (Muhaisen, 2006). The office buildings had depth of $10 \mathrm{~m}$. The variables were glazing systems and window to wall ratio. For glazing they studied the effect of single, double and triple glazing for courtyard walls as well as for atrium skylights. That study also investigated the window to wall ratio $40 \%, 60 \%$ and $80 \%$.

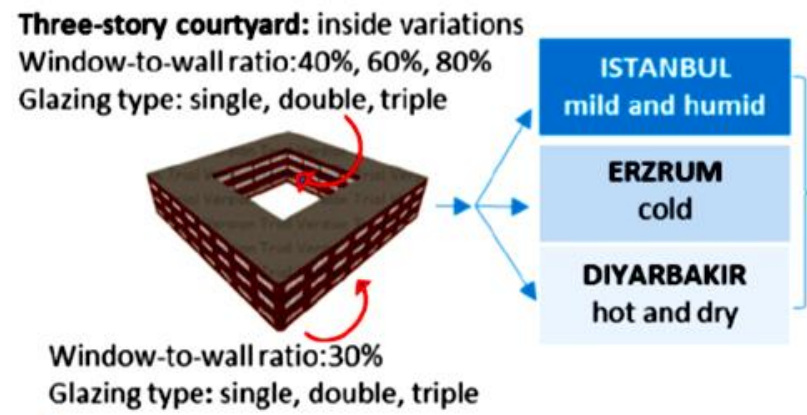

First stage of simulation

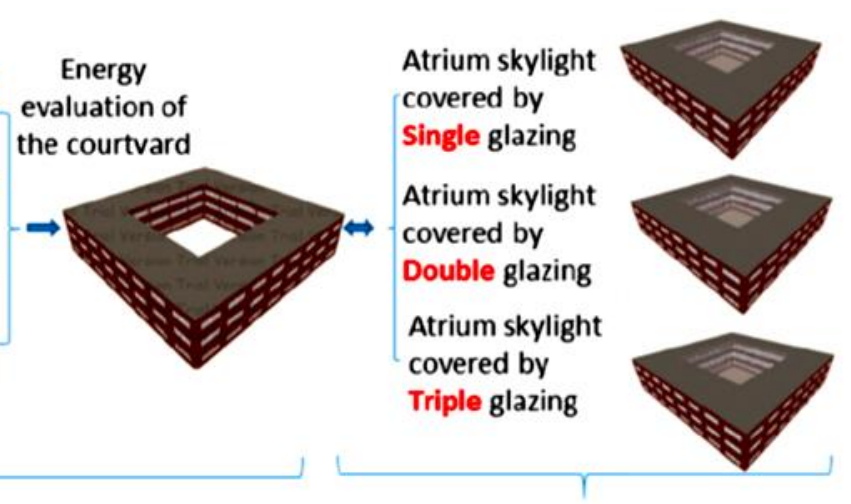

Second stage of simulation

Figure 10 Two stage simulation process (Tabesh \& Sertyesilisik, 2016) 
They concluded that $40 \%$ WWR and triple glazing show best energy performance in all climates. They also found that, integrated usage of courtyard and atrium can save energy consumption of buildings if they are used as courtyard type buildings in summer in all climates and as atrium type buildings in cold months (Tabesh \& Sertyesilisik, 2016).

The study of Aldawoud and Clark (2007) compared the energy performance between courtyard and atrium building in four (4) different climatic regions of USA, like cold, temperate, hot-humid and hot-dry climate. Square plan was used for both courtyard and atrium buildings. Their study used different glazing types and WWR as variables. They concluded that open courtyard houses consume lowest energy when the building is not very tall and with the increase of building height, at certain point atrium shows the better performance. This 'break-even' points for number of stories depend on different factors, like glazing type, climate etc. For example, if the courtyards having $30 \%$ glazing with single clear glass is compared with atrium having $80 \%$ glazing with single clear glass, then courtyard is found more energy efficient option for cooling load until 10 floors in hot dry climate. After this height atrium option is more energy efficient. But in cold climates with the same settings these two building options perform differently. In this case courtyard building is energy efficient for heating load until $5^{\text {th }}$ floor, after that height atrium building is more energy efficient option (Aldawoud \& Clark, 2008).

There are individual studies found on atrium type of buildings. In 2002, Laouadi, A. et al revealed that compared to flat skylight, in cold climate pyramidal/pitched skylight is responsible for $25 \%$ more solar heat gain in linear atria and 3-sided atrium for $10 \%$ more solar heat gain (Laouadi et al., 2002). Study shows that glass height and atrium 


\subsection{Summary of the literature review}

\section{Table 2 Summary of literature review}

\begin{tabular}{|c|c|c|c|}
\hline Topics & Main findings & Methodology & Reference \\
\hline $\begin{array}{l}\text { Courtyard as indoor and } \\
\text { outdoor climate } \\
\text { moderator }\end{array}$ & $\begin{array}{l}\text { Courtyards bring natural light and air inside } \\
\text { the houses and reduce energy consumptions. } \\
\text { It modifies indoor and outdoor climate. }\end{array}$ & $\begin{array}{l}\text { Design builder } \\
\text { software package } \\
\text { for simulation }\end{array}$ & $\begin{array}{l}\text { Fathy, 1986; Oliver, 2003; } \\
\text { Taleghani, Tenpierik, \& } \\
\text { Dobbelsteen, } 2014\end{array}$ \\
\hline $\begin{array}{l}\text { Built potential and } \\
\text { daylighting criteria of } \\
\text { urban generic forms }\end{array}$ & $\begin{array}{l}\text { Among six generic urban forms (pavilions, } \\
\text { slabs, terrace-courts, pavilion-courts and } \\
\text { courts), performance of courtyard form is } \\
\text { best in terms of built potential and daylighting } \\
\text { criteria. }\end{array}$ & $\begin{array}{l}\text { Digital Elevation } \\
\text { Model (DEM) } \\
\text { analyzed with } \\
\text { Matlab image } \\
\text { processing toolbox. }\end{array}$ & $\begin{array}{l}\text { Martin \& March, 1972); } \\
\text { Steemers et al., 1997; Ratti, } \\
\text { Raydan, \& Steemers, } 2003\end{array}$ \\
\hline $\begin{array}{l}\text { Optimum configuration of } \\
\text { courtyard in row houses } \\
\text { in Toronto }\end{array}$ & $\begin{array}{l}\text { Courtyard with larger footprint having } 20 \% \text { to } \\
40 \% \text { window to wall ratio is an optimal } \\
\text { solution for Toronto row houses for getting } \\
\text { more hours of illuminance with less increase } \\
\text { in energy demand. }\end{array}$ & $\begin{array}{l}\text { Energyplus } \\
\text { software for } \\
\text { simulation. }\end{array}$ & Vaisman, 2015 \\
\hline $\begin{array}{l}\text { Comparative study of } \\
\text { courtyards in different } \\
\text { climatic condition }\end{array}$ & $\begin{array}{l}\text { Courtyards in different climate like hot-dry, } \\
\text { hot-humid, temperate and cold climatic } \\
\text { regions of United States were studied. It was } \\
\text { found that, courtyard's thermal performance } \\
\text { is best in hot humid climate and worse in cold } \\
\text { climate. The study found that, for cold } \\
\text { climate, courtyard with } 30 \% \text { glazing and triple } \\
\text { clear glass can be optimum solution. }\end{array}$ & $\begin{array}{l}\text { Models were } \\
\text { created in } \\
\text { DOE2.1E. }\end{array}$ & Aldawoud, 2008 \\
\hline $\begin{array}{l}\text { Cross ventilation in } \\
\text { courtyard }\end{array}$ & $\begin{array}{l}\text { Cross ventilation in courtyard significantly } \\
\text { improves thermal comfort and reduces } \\
\text { cooling load. }\end{array}$ & & $\begin{array}{l}\text { Al-Hemiddi \& Megren Al-Saud, } \\
2001\end{array}$ \\
\hline Courtyard in snow climate & $\begin{array}{l}\text { Courtyards in cold climates protect buildings } \\
\text { from cold winds. }\end{array}$ & $\begin{array}{l}\text { Energyplus } \\
\text { software for }\end{array}$ & $\begin{array}{l}\text { Mänty \& Pressman, 1988; } \\
\text { Vaisman, } 2015\end{array}$ \\
\hline Atrium in cold climate & $\begin{array}{l}\text { In cold climates atriums are intentionally used } \\
\text { to reduce heat loss, capture solar energy, } \\
\text { bringing daylight inside the houses. }\end{array}$ & $\begin{array}{l}\text { Design builder } \\
\text { software package } \\
\text { for simulation }\end{array}$ & $\begin{array}{l}\text { Ayoob \& Izard, 1994; Edwards } \\
\text { et al., 2006; Laouadi, Atif, \& } \\
\text { Galasiu, 2003; Tabesh \& } \\
\text { Sertyesilisik, } 2016\end{array}$ \\
\hline $\begin{array}{l}\text { Performance of hybrid } \\
\text { system in temperate } \\
\text { climate }\end{array}$ & $\begin{array}{l}\text { Hybrid system (courtyard and atrium } \\
\text { together) reduces energy consumption and } \\
\text { increases comfort hours in temperate climate } \\
\text { like The Netherlands. }\end{array}$ & $\begin{array}{l}\text { Design builder } \\
\text { software package } \\
\text { for simulation }\end{array}$ & Taleghani et al., 2014 \\
\hline $\begin{array}{l}\text { Timing for use of hybrid } \\
\text { system }\end{array}$ & $\begin{array}{l}\text { Integrated use of hybrid system (courtyard } \\
\text { and atrium) can save energy consumption of } \\
\text { buildings if courtyard system is used in } \\
\text { summer in all climates and atrium is used in } \\
\text { colder months. }\end{array}$ & $\begin{array}{l}\text { Design builder } \\
\text { software package } \\
\text { for simulation }\end{array}$ & Tabesh \& Sertyesilisik, 2016 \\
\hline $\begin{array}{l}\text { Building height vs } \\
\text { courtyard and atrium's } \\
\text { performance }\end{array}$ & $\begin{array}{l}\text { open courtyard thermally performs better in } \\
\text { shorter building, but with the increase of } \\
\text { building height at certain point atrium } \\
\text { performs better. }\end{array}$ & $\begin{array}{l}\text { Models were } \\
\text { created in } \\
\text { DOE2.1E. }\end{array}$ & Aldawoud \& Clark, 2008 \\
\hline
\end{tabular}




\subsection{Objective and Research question}

Toronto is characterized by semi continental climate which experiences significant warm humid summer along with prolonged cold winter. In Vaisman study, it is found that in Toronto row houses, introduction of court can bring daylight inside the house with the increased energy load during winter. Literatures show that to reduce yearly energy consumption for heating and cooling inside the house hybrid system can be beneficial (courtyard option in the house during summer, conversion of courtyard into atrium during winter). But these studies were done for temperate and cold climates. However, what would be the impact if we convert this court into atrium during winter in semi continental climate like Toronto is not studied yet.

So, my research intends to extend German Vaisman (2015) research with hybrid system i.e. use of atrium in winter and open courtyard in summer in row houses of Toronto to understand:

- How the energy performance and daylighting conditions are impacted in a row house with courtyard in Toronto if the courtyard is converted into atrium during winter?

- How to maximize the benefits through design decisions in row house with atrium by minimizing energy consumption and increasing daylighting during winter in Toronto? 


\subsection{Expected Results}

The outcome of this study will support to:

- Develop context specific design guidelines - for new constructions and retrofits of row houses

- Contribute to the possible passive design strategies - for improved energy performance

- Enhance indoor comfort environment (thermal comfort and daylighting condition) and create opportunity for extended outdoor hours for recreational activities - as health benefit

\section{Methodology}

\subsection{Introduction}

To answer the research question of this research, i.e. the implication of hybrid system (courtyard and atrium option) in the typical row houses of Toronto this research follows the steps shown in figure below.

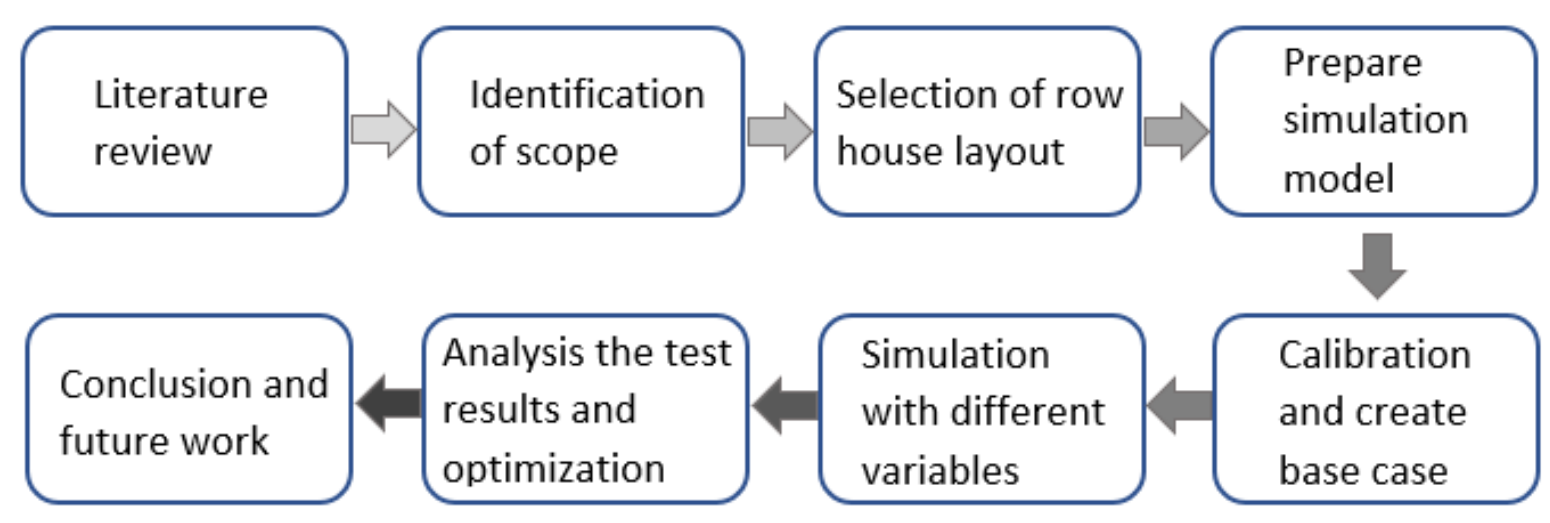

Figure 11 Research Process 


\subsection{Building layout of Toronto row house}

For this research, an existing row house of Toronto has been used. This retrofitted Toronto row house is adopted from German Vaisman (2015). This house represents the typical row houses in Toronto in size and massing (Vaisman, 2015). Moreover, as Vaisman's simulation were calibrated against the actual energy bills / usage of the family, it will be possible to indirectly verify our findings against them, too.

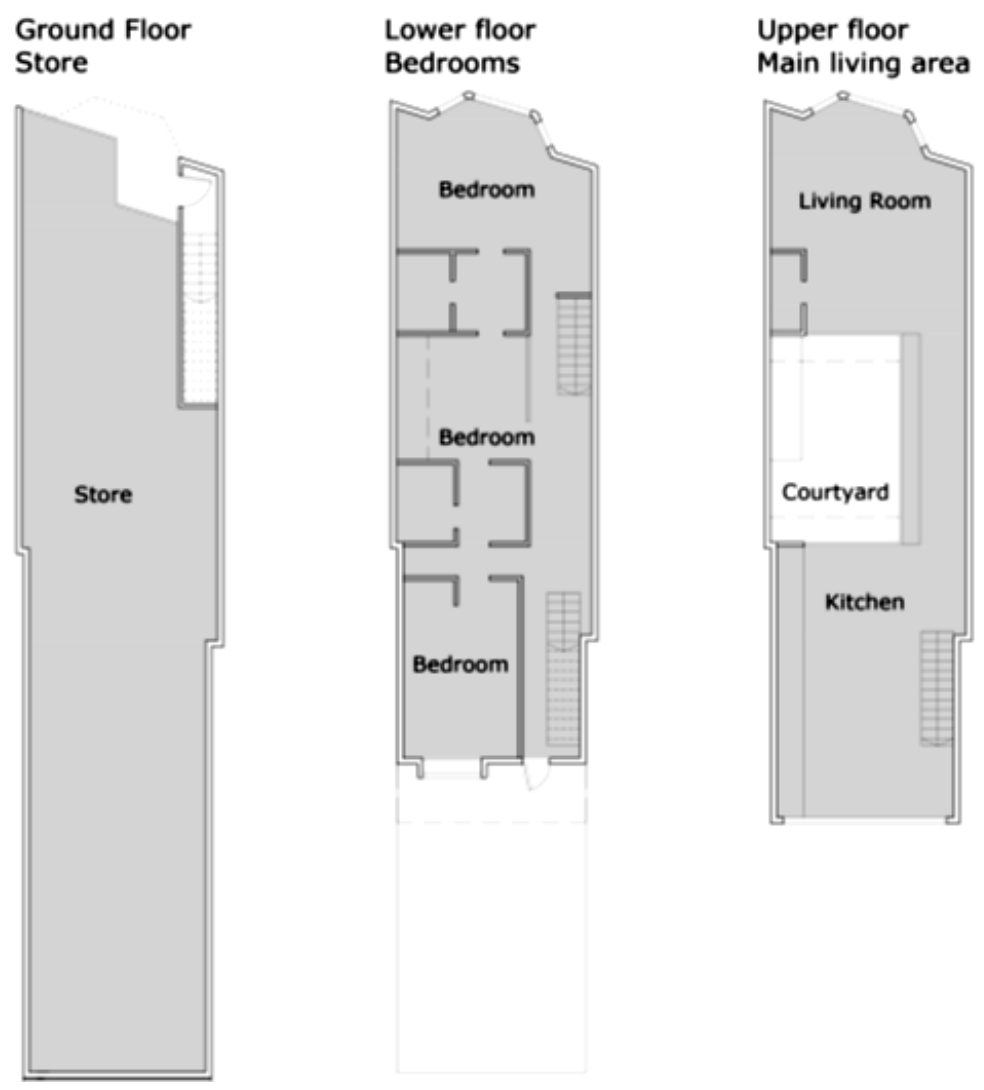

Figure 12 Existing house plan (Vaisman, 2015) 

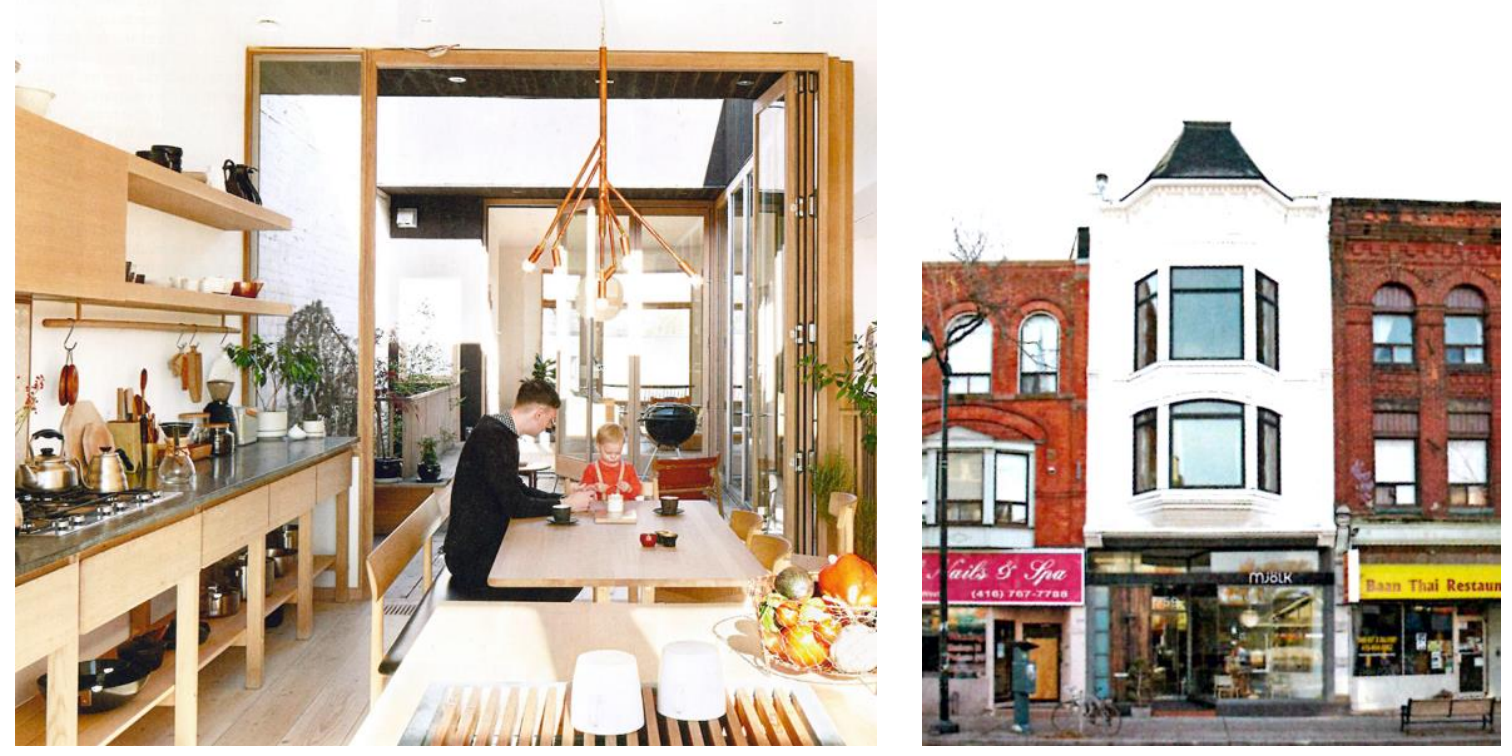

Figure 13 Interior and exterior of case study row house (Vaisman, 2015)

This 3-story house was originally built in 1889. On the $1 \mathrm{st}$ floor there is a retail shop and the dwelling unit is on 2nd and 3rd floor. The floor areas of 2 nd and 3rd floors are $81 \mathrm{~m} 2$ (872 sft) and $87 \mathrm{~m} 2$ (937 sft) respectively. The $1 \mathrm{st}$ floor is $118 \mathrm{~m} 2$ (1270 sft) and not included in simulation (Vaisman, 2015).

\subsection{Simulation layout and orientation}

In simulation process minor geometric simplification can be made for reducing the number of surfaces and ease the simulation without reducing the building surface area (Zirnhelt, 2013). In this research a simplified drawing of the original layout adopted from Vaisman (2015). This simplification mainly done on North façade by straitening the floor. 


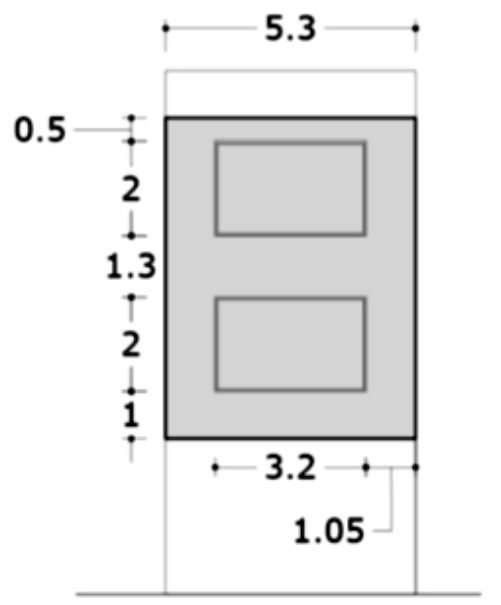

Front Elevation
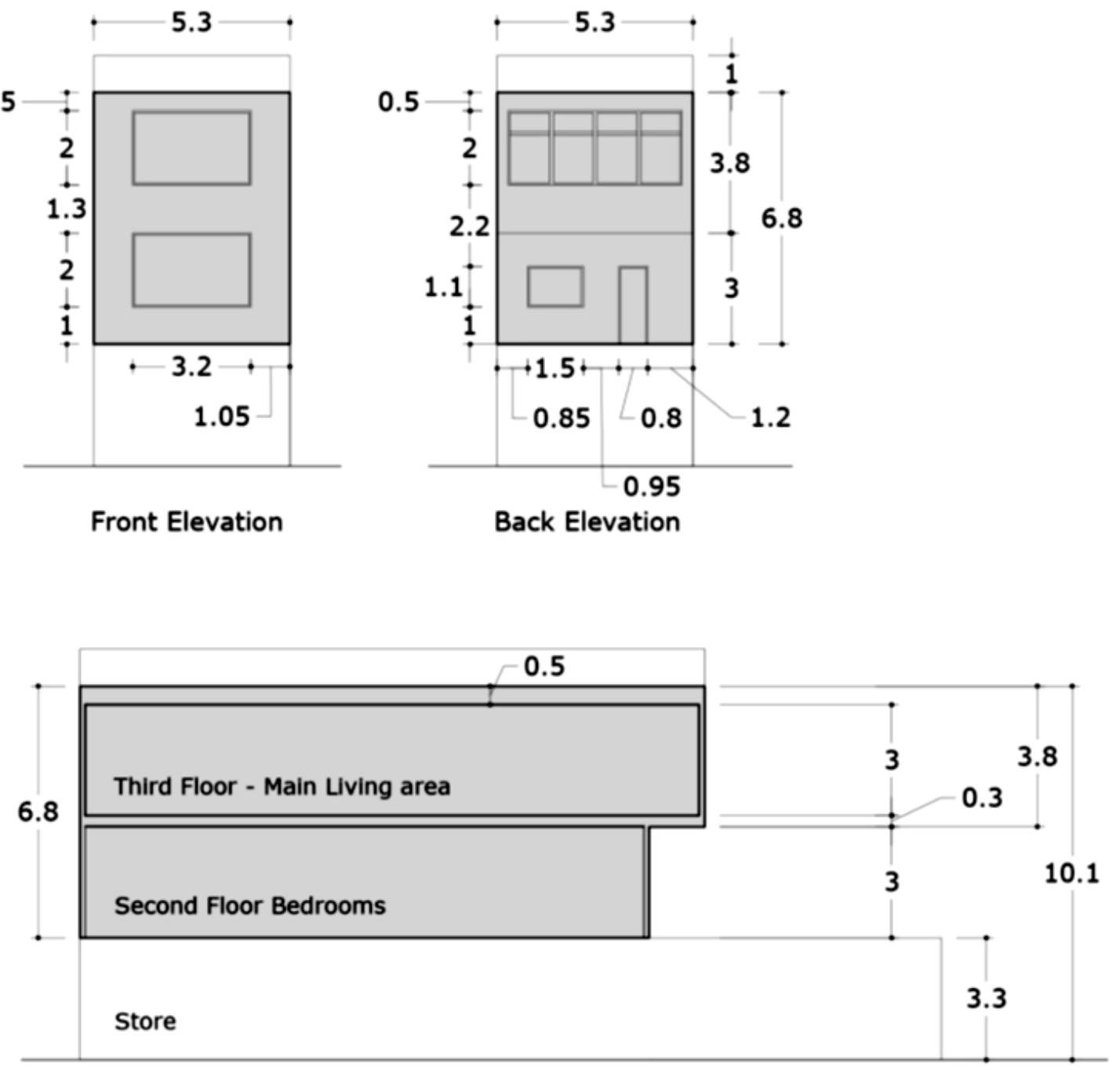

\section{Section}

Figure 14 Elevations and section of the existing house. Units are in meters (Vaisman, 2015) 
Lower Floor Rooms

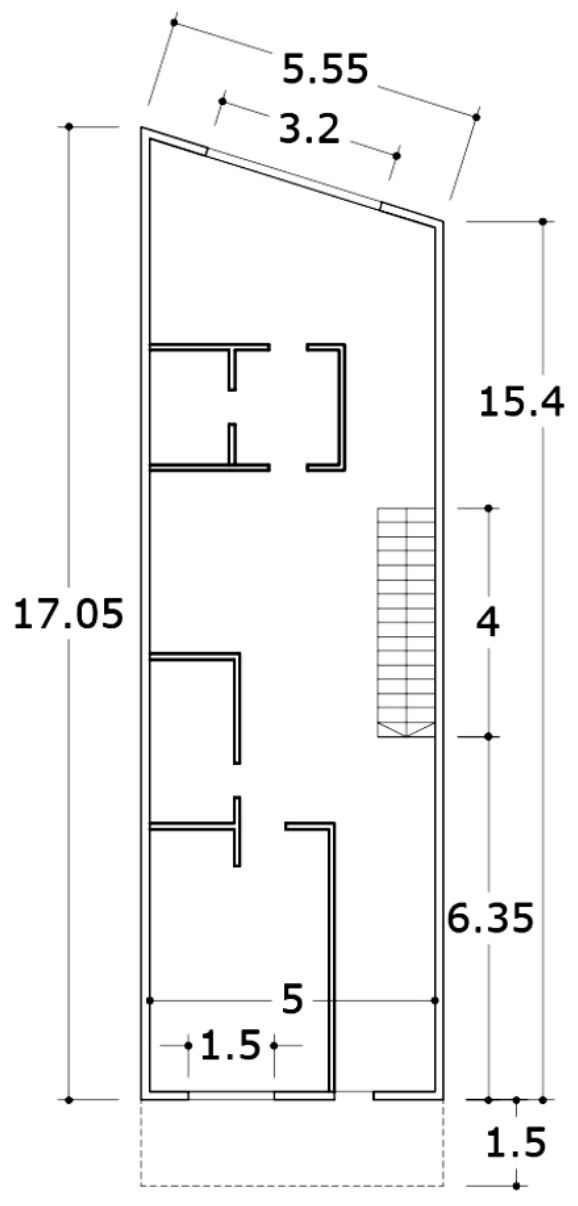

Upper Floor Living Areas

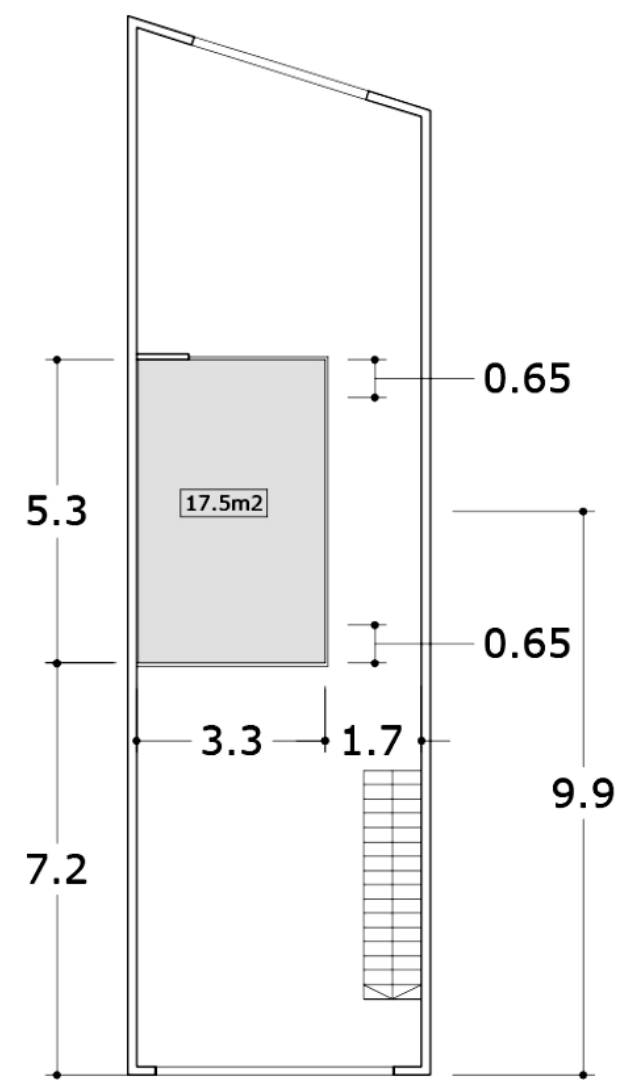

0.5

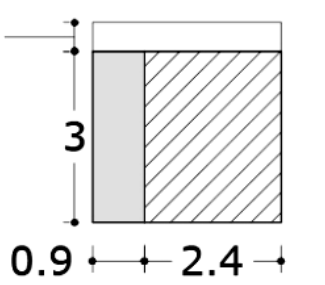

0.65

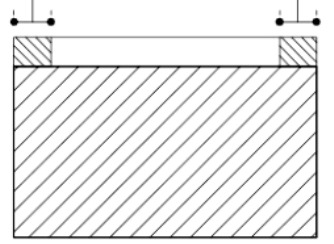

5.3
0.65

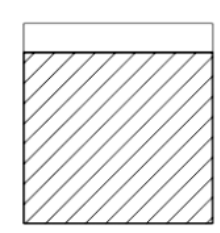

$3.3 \longrightarrow$

\section{ELEVATION REFERENCES}

VIIA Glass

बMU Overhang section

Figure 15 Simplified plans and elevations of the existing house. Units are in meters (Vaisman, 2015) 
In the simulation model, a single zone is created on each floor and they are openly connected through stairwell. In some research simulations, stairwells are modeled as glass surfaces with high transmittance, high longwave transmittance, high emissivity and very low thermal resistance since omitting a portion of a wall or floor surface also omits heat transfer associated with that surfaces (Jermyn, 2014; Vaisman, 2015; Zirnhelt, 2013).

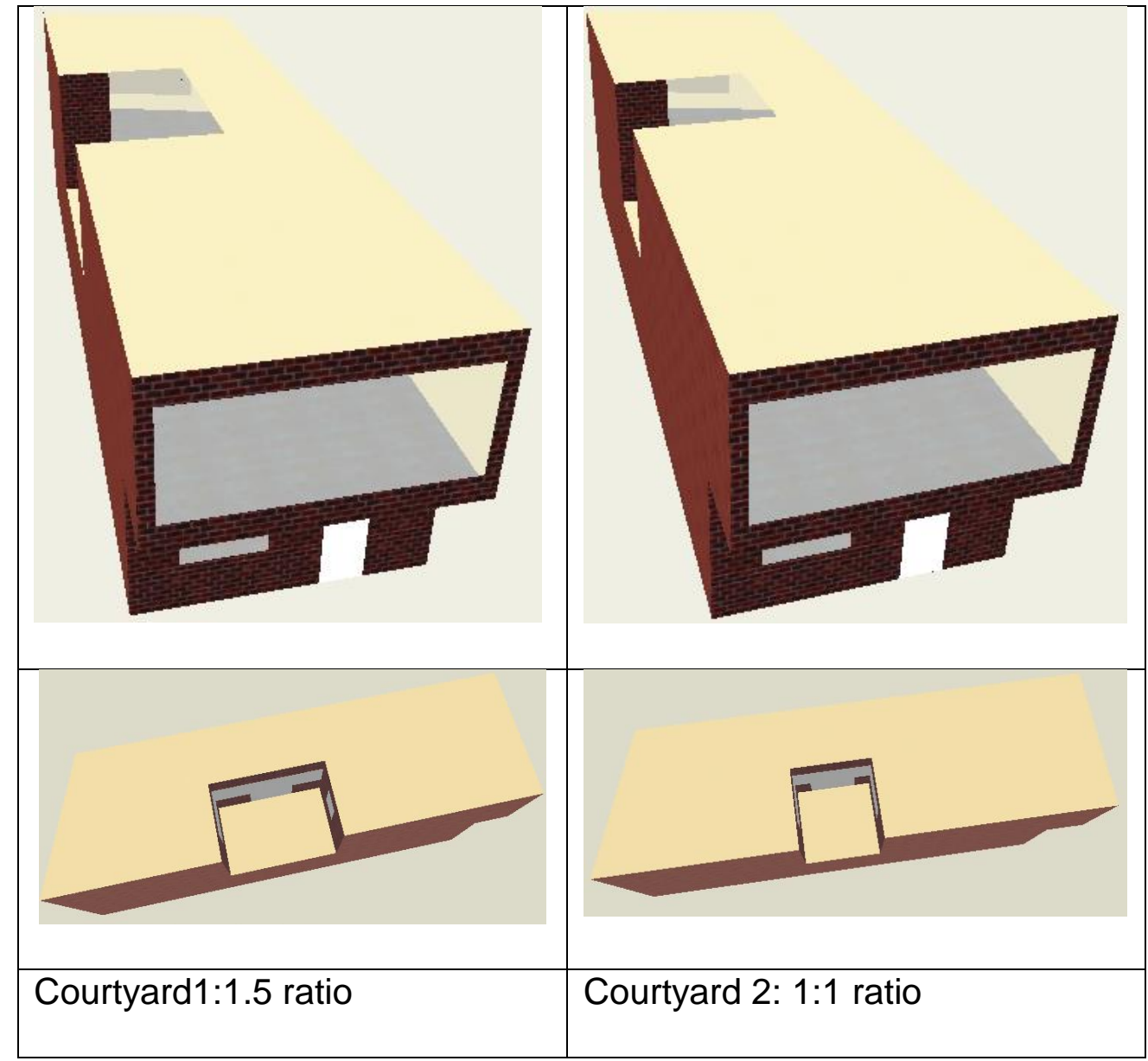

Figure 16 Courtyard configurations

A single-story courtyard is created in the row house as stated by Vaisman (2015). It is assumed that the case study row house is one of the middle units, that means there are other row house units on the both sides. So, it is logical to show that this courtyard is 
surrounded by four walls. If wall is omitted from one side of the courtyard which is attached to other row house unit, that may affect energy consumption and daylighting differently than actual situation. But in that case simulation software considers that courtyard as interior space. So, in this research to create courtyard as open space for simulation, one side of the courtyard is kept open. After simulation, it was reassured that software considers this courtyard as open space as temperature profile of courtyard is similar to outdoor temperatures.

On north and south façade windows and doors are placed with actual dimensions and on actual locations. North and south façades are considered as external façades. But facades attached to the other units are considered as adiabatic. The floor between $1 \mathrm{st}$ floor retail store and 2 nd floor dwelling is also considered as adiabatic floor. To simulate atrium, skylight is considered over courtyard.

\subsection{Courtyard size, orientation and glazing percentage}

Vaisman (2015) showed that small courtyards in row houses were not optimal solutions for bringing daylight inside the house. Bigger size courtyards $(4.5 \mathrm{~m} \times 3 \mathrm{~m}$ and $3 \mathrm{~m} \times 3 \mathrm{~m})$ with $30 \%$ to $40 \%$ glazing can be optimum solution for bringing daylight inside the house with moderate increase in energy consumption (Vaisman, 2015). Therefore, this research will use two large courtyard configurations with 4 different glazing percentages, exactly as they were designed by Vaisman (2015). All these models used for north, east, south and west orientations, resulting in total of 32 analyzed models. North, east, south and west direction means orientation of courtyards towards that directions as shown in figure below. 


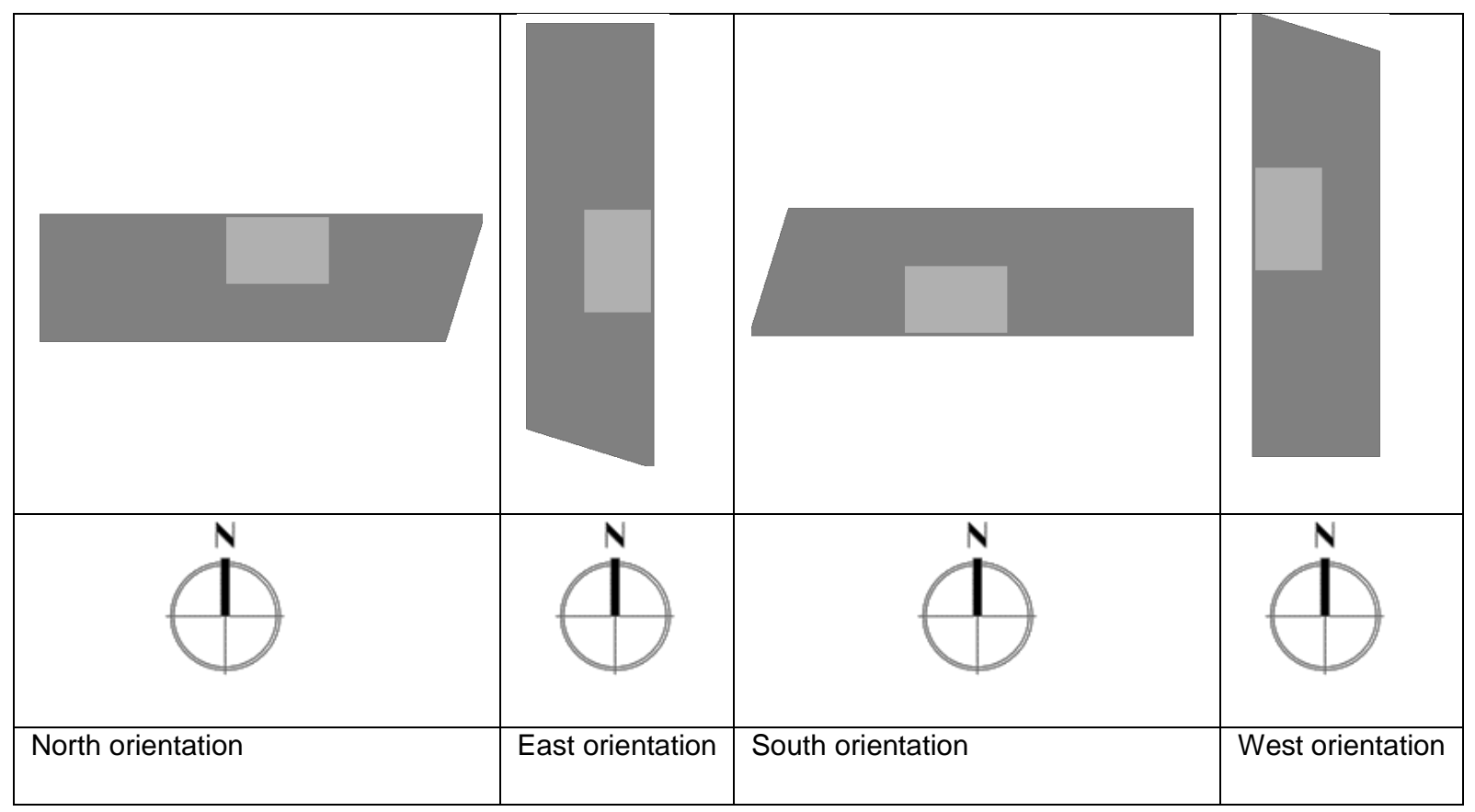

Figure 17 Orientation of courtyards

So total simulation modelling options are like this:

Table 3 Simulation modeling options

\begin{tabular}{|c|c|c|c|c|}
\hline \multicolumn{5}{|c|}{ Courtyard type -1 (1:1.5 - 4.5m X 3m) } \\
\hline Glazing & \multicolumn{4}{|c|}{ Orientation } \\
\hline $77 \%$ & \multirow{4}{*}{ North } & \multirow{4}{*}{ East } & \multirow{4}{*}{ South } & \multirow{4}{*}{ West } \\
\hline $57 \%$ & & & & \\
\hline $36 \%$ & & & & \\
\hline $20 \%$ & & & & \\
\hline \multicolumn{5}{|c|}{ Courtyard type $-2(1: 1-3 m \times 3 m)$} \\
\hline Glazing & \multicolumn{4}{|c|}{ Orientation } \\
\hline $73 \%$ & \multirow{4}{*}{ North } & \multirow{4}{*}{ East } & \multirow{4}{*}{ South } & \multirow{4}{*}{ West } \\
\hline $55 \%$ & & & & \\
\hline $36 \%$ & & & & \\
\hline $23 \%$ & & & & \\
\hline
\end{tabular}


Courtyard \#1 Rectangular $1: 1.5$
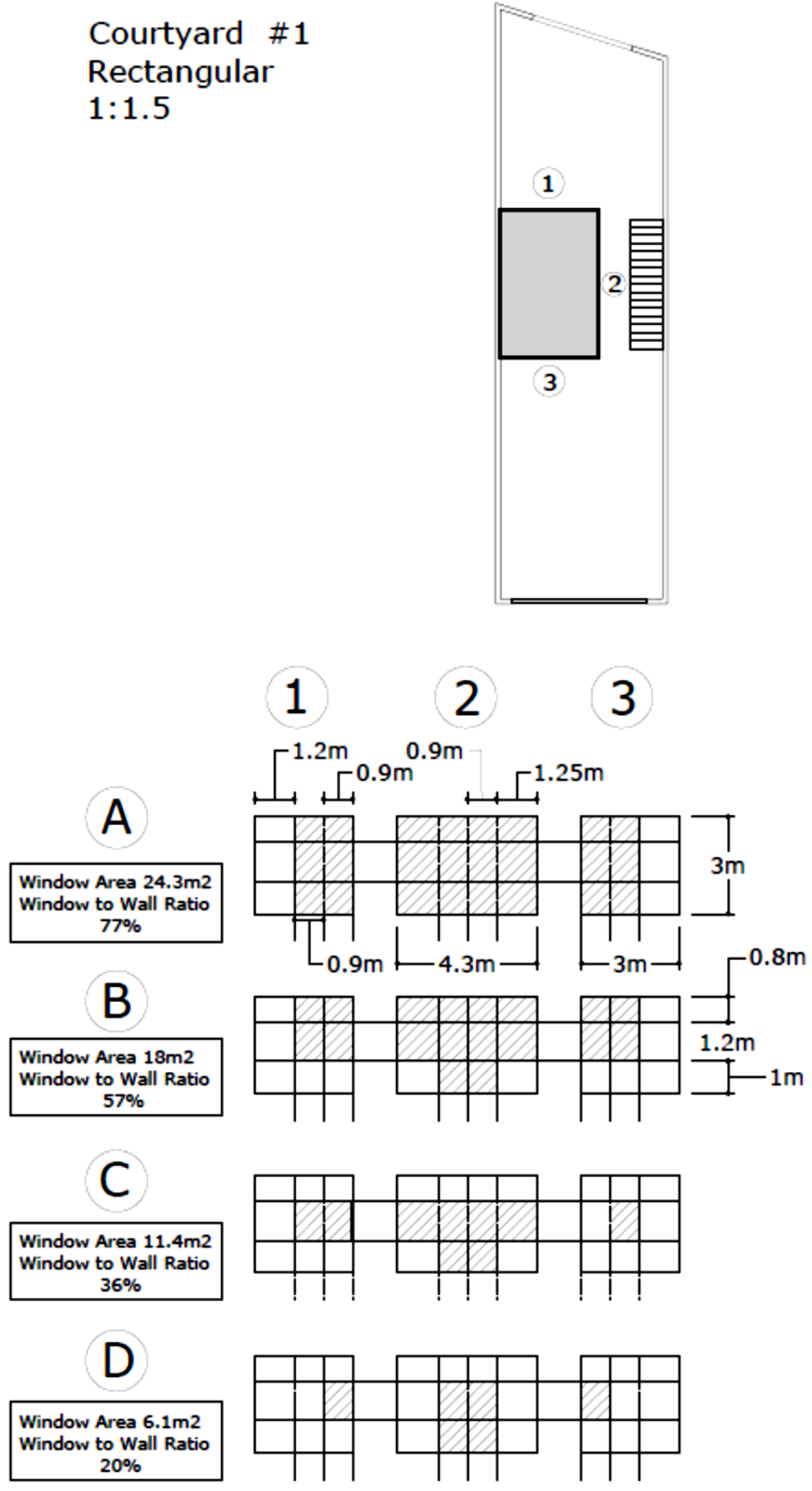

Figure 18 Courtyard configuration -1 (Vaisman, 2015) 


\section{Courtyard \#2 Squared \\ $1: 1$}
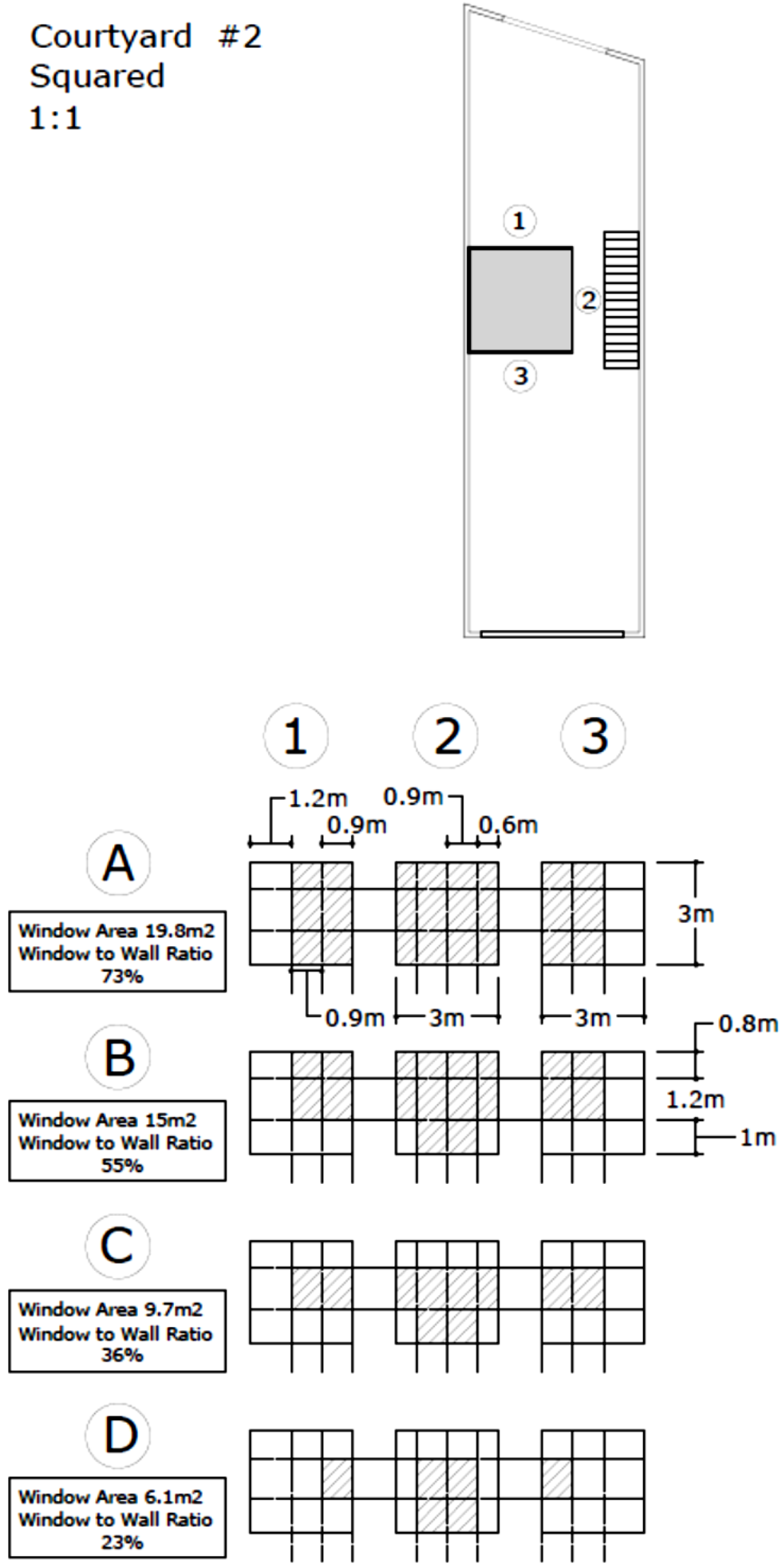

Figure 19 Courtyard configuration 2 (Vaisman, 2015) 


\subsection{Simulation software}

Vaisman (2015) modeled the row house in Sketchup using Open Studio plug-in. It was analyzed in Energy Plus software for flexibility of detail input and accuracy (Crawley, D., \& et al., 2004; Vaisman, 2015).

For this research Design Builder Software package (Version 4.2.0.054) has been used. It is one of the most comprehensive simulation tools available today. A simplified CAD interface, templates, wizards and other configurations of energy plus included in Design Builder as it employs the state-of-the-art building performance simulation engine of Energy Plus (Tabesh \& Sertyesilisik, 2016; Taleghani et al., 2014). This comprehensive simulation tool ensures detailed accounting of energy inputs and energy loses and considers solar heat gains through glazing systems, detail heat conduction and convection between different zones. It also provides variety of country or region-specific templates for easing the selection process of parameters. Energy use or extract by HVAC systems also easily definable by these templates (DesignBuilder software guide, 2009; Tabesh \& Sertyesilisik, 2016; Taleghani et al., 2014).

Daylight control is possible in Design Builder. That means it is possible to control electric lights according to the availability of the natural daylight at expected illuminance level. It calculates the daylight illuminance using many possible factors like - sun position, sky condition, season, location, type and size of glazing etc. Advanced Delight calculation method using ray-tracing to calculate the daylight illuminance is possible in Design Builder (DesignBuilder software guide, 2009). Using the BESTest (Building Energy Simulation TEST) procedure, which is originally developed by the International Energy Agency, the accuracy of the Design Builder has been validated (Radhi, 2010). 
For constructing the geometrical model, it was decided to construct the model in Design Builder itself due to the difficulties in comparability of the exporting and importing plugins, whereas Design Builder provided the most accurate option.

\subsection{Weather file}

Energy and environmental performance of building are greatly influenced by climatic condition of a region. Energy consumption of a building is uniquely challenged by its climate (Tabesh \& Sertyesilisik, 2016). So, it is quite important to choose appropriate weather file for building energy simulation. Design Builder comes with a database of hourly weather data which uses hourly weather information to define external condition during simulations. For this research from the built-in list of Design Builder 'Toronto Int'। ON CAN WYEC2' - weather file is selected for the location of Toronto. It follows ASHRAE WYEC2 (Weather Year for Energy Calculation 2) format. The hourly weather information for Canada developed from the record of the period 1953-1995 in the Canadian Energy and Engineering Data Sets (CWEEDS) (Vaisman, 2015).

\subsection{Simulation input parameters}

Careful selection of input parameters is essential for any energy simulation. Energy simulation can be significantly affected by many input parameters (Zirnhelt, 2013). To develop the base case, this research considered all the input parameters used by Vaisman (2015). But all the input parameters required for detail input in Design Builder were not available in that literature. So, this research used other literatures, guidelines and standards. Input parameters were either taken directly from their recommended values or by best assumptions possible from them. The parameters that are not listed in 
the table below are kept default as appears in Design Builder software for location and typology specific building due to lack of available information. But for this type of input, literature review was done to ensure that they do not impact energy consumption significantly. Uncertain parameters like: internal gain for appliances, air leakage (ac/h) were adjusted (increased or decreased the value to get the closer value to the reference research) at the calibration stage.

For the simulation, firstly, location related parameters were set. After selecting the location from the default list longitude and latitude for the location were automatically populated. But the elevation was set as $173 \mathrm{~m}$ for this specific building from Vaisman (2015). For the regulatory guidelines for energy usage in residential buildings 'Canadian Energy Code (homes)' is selected from default list.

Heating set point fixed at $21^{\circ} \mathrm{C}$ and cooling set point at $24^{\circ} \mathrm{C}$ with one centrally located thermostat in the upper floor (Vaisman, 2015). The Illuminating Engineering Society of North America (IESNA) recommends 100 lux of illuminance for those working places where simple visual tasks are performed. Vaisman (2015) set these 100 lux for Toronto row house study to calculate the hours of illuminance inside the house (Cuttle, 2003; Vaisman, 2015). This study also uses this value as target illuminance for the simulation. Lighting control turned on to consider the energy saving by turning off the artificial lighting in places inside the house where illuminance level reaches 100 lux with day lighting.

For construction input parameters, this research utilized the layers used by Vaisman (2015). He derived that information from the Ontario Building Code (OBC) 
Supplementary Standard SB-12 for building code requirements for low rise - single family residential buildings. This standard contains prescriptive compliance packages with specifications to reach an energy performance improvement. Compared to OBC2006, this improvement estimated 30\% (OBC, 2012; Vaisman, 2015). For the wall and ceiling assembly SB-12 package and construction assembly as follow:

Table 4 SB-12 Package A requirements and specified material for the wall assembly

\begin{tabular}{|c|c|c|c|}
\hline Component & Required & Specified- Nominal RSI & Total Wall Thickness \\
\hline \multirow{2}{*}{ Wall } & \multirow{2}{*}{ 4.23 W/m2.K (R24) } & $4.23 \mathrm{~W} / \mathrm{m} 2 . \mathrm{K}-0.16 \mathrm{~m}$ & \multirow{2}{*}{$0.24 \mathrm{~m}$} \\
\hline & & Fiberglass & \\
\hline \multirow{2}{*}{ Roof } & \multirow{2}{*}{$5.46 \mathrm{~W} / \mathrm{m} 2 . \mathrm{K}(\mathrm{R} 31)$} & $5.46 \mathrm{~W} / \mathrm{m} 2 . \mathrm{K}-0.21 \mathrm{~m}$ & \multirow{2}{*}{$0.29 \mathrm{~m}$} \\
\hline & & Fiberglass & \\
\hline \multirow{2}{*}{ Exposed floor } & \multirow{2}{*}{$5.46 \mathrm{~W} / \mathrm{m} 2 . \mathrm{K}(\mathrm{R} 31)$} & $5.46 \mathrm{~W} / \mathrm{m} 2 . \mathrm{K}-0.21 \mathrm{~m}$ & \multirow{2}{*}{$0.50 \mathrm{~m}$} \\
\hline & & Fiberglass & \\
\hline
\end{tabular}

Table 5 Construction assemblies

\begin{tabular}{|l|l|l|l|}
\hline Exterior Walls & Roof & Window & Door \\
\hline Brick $100 \mathrm{~mm}$ & Shingle & Glass $6 \mathrm{~mm}$ & $2.1 \mathrm{~m} 2 . \mathrm{K} / \mathrm{W}$ \\
\hline Fibreglass $160 \mathrm{~mm}$ & OSB & Air gap $13 \mathrm{~mm}$ & \\
\hline Brick $100 \mathrm{~mm}$ & Fibreglass $210 \mathrm{~mm}$ & Glass $6 \mathrm{~mm}$ & \\
\hline Plaster $12.7 \mathrm{~mm}$ & Plaster $12.7 \mathrm{~mm}$ & & \\
\hline
\end{tabular}

For the construction Air tightness was initially considered $1.5 \mathrm{ac} / \mathrm{h}$ as Zirnhelt (2013) used that value for his calibrated simulation for Canadian Tract House (Zirnhelt, 2013). But this value was adjusted during calibration. Both Vaisman (2015) and Zirnhelt (2013) used the same double-glazed window with $12 \mathrm{~mm}$ air gap, specific transmittance, reflectance and emissivity data in Energy Plus. In this research 'Double clear $6 \mathrm{~mm} / 13$ $\mathrm{mm}$ air' glazing system used from Design Builder default list for base case and its specification found almost same as used by Vaisman (2015) and Zirnhelt (2013). 
For internal gains: lighting power density, occupancy and appliances are usually considered in simulation process. In this research target illuminance is set as 100 lux. Vaisman (2015) also considered to use LED lights as the source of artificial lighting. However, in Design Builder there is no option was found to set the lighting typology as LED. The impact of LED can be converted into lighting power density. Both Vaisman (2015) and Zirnhelt (2013) calculated lighting consumption $1.93 \mathrm{~W} / \mathrm{m}^{2}$ in the main floor (i.e. living room) are and $3.07 \mathrm{~W} / \mathrm{m}^{2}$ for the lower floor bed rooms (Vaisman, 2015; Zirnhelt, 2013). Vaisman (2015) also considered 4 occupants (2 parents and 2 children) in the residence. For the lighting power density and number of occupants this research follows Vaisman (2015) and Zirnhet (2013)'s assumptions. For the household appliances there is no specific data was found from the above-mentioned researches. However, it is difficult to assume what type of appliances are exactly used (like, stove, dryer, washer, fridge, dishwasher, vacuum cleaner etc.) without physical investigation to that house. Therefore, to get the idea for appliances' load ASHRAE manual and other literatures were consulted. Initially the value $2.5 \mathrm{~W} / \mathrm{m} 2$ was assumed for common household appliances (Issi \& Kaplan, 2018). This value was adjusted during calibration.

Previous research simulations for Toronto residences used 'Typical residential forcedair gas furnace system' with centrally located thermostat (Jermyn, 2014; Vaisman, 2015; Zirnhelt, 2013). Vaisman (2015) also used the reference of OBC SB-12 which requires the furnace minimum annual fuel utilization efficiency (AFUE) $90 \%$ for natural gas furnace (OBC, 2012; Vaisman, 2015). In Design Builder Typical residential forced air gas furnace system could not be applied. After consulting literatures and discussion with Design Builder users' online group at unmethours.com, this is substituted by 
another close option. Residential forced air gas furnace system - which has centrally heated air in the winter, centrally cooled air in summer, constant volume, constant fresh air volume and single thermostat can be replaced by HVAC template 'Constant volume Direct Expansion (unitary multizone) in Design Builder. Thus, this research was used 'Constant Volume DX' as HVAC template. The list of input parameters is as follow:

\section{Table 6 Base case input parameters}

\begin{tabular}{|c|c|c|c|}
\hline \multicolumn{4}{|c|}{ Building Specific Parameters for verification Energy Models } \\
\hline Parameters & \multicolumn{2}{|c|}{ Input } & Reference \\
\hline Archetypes & \multicolumn{2}{|c|}{ Toronto Row House } & Vaisman, 2015 \\
\hline \multirow{5}{*}{ Location } & Weather file & \multicolumn{2}{|c|}{ CAN_ON_TORONTO INT'L_WYEC2 Vaisman, 2015} \\
\hline & Latitude [deg] & 43.67 & Vaisman, 2015, Weather file \\
\hline & Longitude [deg] & -79.4 & Vaisman, 2015, Weather file \\
\hline & ASHRAE climate zone & $6 \mathrm{~A}$ & Default from weather file \\
\hline & Elevation & $173 \mathrm{~m}$ & Vaisman, 2015 \\
\hline Energy code & \multicolumn{2}{|c|}{ Candian Energy Code (Homes) } & Assumption \\
\hline Heated Floor Area & \multicolumn{2}{|c|}{$179.9 \mathrm{~m} 2$} & Vaisman, 2015 and software \\
\hline Storeys & \multicolumn{2}{|c|}{2} & Vaisman, 2015 \\
\hline \multirow{3}{*}{ Environmental control } & Heating setpoint (OC) & 21 & Vaisman, 2015 \\
\hline & Cooling setpoint (OC) & 24 & Vaisman, 2015 \\
\hline & Target illuminance & 100 Lux & Vaisman, 2015; Cuttle, 2003 \\
\hline \multirow{10}{*}{ Construction } & \multirow{4}{*}{ Ceilling Assembly } & Shingle & \multirow{4}{*}{ Vaisman, 2015; OBC SB-12, 2012} \\
\hline & & OSB & \\
\hline & & Fibreglass $210 \mathrm{~mm}$ & \\
\hline & & Plaster 12.7 mm & \\
\hline & \multirow{4}{*}{ Wall Assembly } & Brick $100 \mathrm{~mm}$ & \multirow{4}{*}{ Vaisman, 2015; OBC SB-12, 2012} \\
\hline & & Fiberglass $160 \mathrm{~mm}$ & \\
\hline & & Brick $100 \mathrm{~mm}$ & \\
\hline & & Plaster $12.7 \mathrm{~mm}$ & \\
\hline & Internal Floor & Dense cast concrete $100 \mathrm{~mm}$ & Default in software \\
\hline & $\begin{array}{l}\text { Air Leakage (ac/h under } \\
\text { standard operating } \\
\text { pressure) }\end{array}$ & 1.32 & Zirnhelt, 2013 and adjusted \\
\hline \multirow{2}{*}{ Opening } & Window Type & Double Clr $6 \mathrm{~mm} / 13 \mathrm{~mm}$ Air & Vaisman, 2015 \\
\hline & Door Type & Insulated & Vaisman, 2015 \\
\hline HVAC System & \multicolumn{2}{|c|}{ Constant Volume Direct Expansion } & Vaisman, 2015, Jermyn, 2014 and adjusted \\
\hline \multirow{3}{*}{ Internal Gains } & Lighting & 1.9 and $3.07 \mathrm{~W} / \mathrm{m} 2$ & Vaisman, 2015; Zirnhelt, 2013 \\
\hline & Appliances & $1.8 \mathrm{~W} / \mathrm{m} 2$ & Issi, 2018 and adjusted \\
\hline & Occupancy & 4 (34 m2/per person) & Vaisman, 2015 \\
\hline
\end{tabular}

\subsection{Base case simulation and calibration}

Calibrate the initial base case is essential to ensure results are close to reference

results. It is especially required when input parameters or simulating software are changed. By this approach energy savings can be quantified from previously 
established energy consumption data without doing a full-scale experiment with reasonable accuracy (Zirnhelt, 2013).

Calibration process is a highly iterative process since error and uncertainty are unavoidable in simulation process and for that reason it either overestimates or underestimates the energy consumption. Jermyn, D. (2014) conducted a simulation in Energy Plus and tried to validate his base case with previously developed similar type of simulation in HOT2000 by Blaszak and Richman (2013). Despite similar input parameters, he found more than $50 \%$ difference in energy consumption result due to difference in energy modelling software and methodology (Jermyn, 2014).

In this research after the first simulation in Design Builder for base case, the result was tried to match with previously established simulation result of German Vaisman (2015) done in Energy Plus. Yet, there is a significant difference was found for 32 base case simulations' energy consumptions which ranges from $4 \%$ to $20 \%$. It was probably due to difference in simulation software and for some assumed, uncertain parameters. Therefore, this research tries to identify the significance of uncertain parameters on energy consumption results from relevant literatures. It was found that internal gain through appliances, air tightness of construction etc. can have large influence on energy consumption (Blaszak, 2010; Jermyn, 2014; Zirnhelt, 2013). For these reasons, the simulation model was calibrated adjusting these 2 parameters: appliances' internal gain and air tightness of construction.

It was found that power density (appliances) $-1.63 \mathrm{~W} / \mathrm{m}^{2}$ and airtightness value ac/h0.74 give closer values for both courtyard types with higher percentage of glazing. But 
power density $-1.32 \mathrm{~W} / \mathrm{m}^{2}$ and Airtightness value ac/h -0.6 give more accurate result

for both courtyard types with lower percentage of glazing. So, power density $-1.32 \mathrm{~W} / \mathrm{m}^{2}$

and Airtightness value ac/h -0.6 were finally taken for base case simulation.

Table 7 Courtyard 1 with Power density $1.63 \mathrm{~W} / \mathrm{m} 2$ and ac/h 0.74

\begin{tabular}{|c|c|c|c|c|c|c|c|c|c|c|c|c|c|c|c|c|c|c|}
\hline \multicolumn{3}{|c|}{\begin{tabular}{|l|l|} 
PD-1.63 W/m2 & ACH-0.74 \\
\end{tabular}} & & & & & & & & & & & & & & & & \\
\hline \multicolumn{3}{|c|}{ Courtyard type -1 (1:1.5) } & North & & & & East & & & & South & & & & West & & & \\
\hline \multicolumn{3}{|c|}{ Year round energy } & Elec(kWh) & Diff. (\%) & Gas(kWh) & Diff. (\%) & Elec(kWh) & Diff. (\%) & Gas(kWh) & Diff. (\%) & Elec $(\mathbf{k W h})$ & Diff. (\%) & Gas(kWh) & Diff. (\%) & Elec(kWh) & Diff. (\%) & Gas(kWh) & Diff. (\%) \\
\hline \multirow{8}{*}{ Glazing } & \multirow{8}{*}{$\begin{array}{c}\text { Dble clr, } \\
6 \mathrm{~mm} / 13 \\
\mathrm{~mm} \text { air }\end{array}$} & $77 \%$ & 5073.12 & \multirow{2}{*}{0} & \begin{tabular}{|l|}
17170.7 \\
\end{tabular} & \multirow{2}{*}{0} & 4925.95 & \multirow{2}{*}{-3} & \begin{tabular}{|l|}
17373.6 \\
\end{tabular} & \multirow{2}{*}{-7} & 5013.66 & \multirow{2}{*}{2} & \begin{tabular}{|l|}
17737.6 \\
\end{tabular} & \multirow{2}{*}{6} & 4939.43 & \multirow{2}{*}{-4} & 17093.5 & \multirow{2}{*}{-2} \\
\hline & & Base & 5072 & & 17114 & & 4778 & & 16183 & & 5114 & & 18925 & & 4736 & & 16836 & \\
\hline & & $57 \%$ & 5089.33 & \multirow{2}{*}{-2} & 17180.3 & \multirow{2}{*}{-1} & 4939.59 & \multirow{2}{*}{-10} & 17393.4 & \multirow{2}{*}{-4} & 5029.28 & \multirow{2}{*}{-1} & 17750.6 & \multirow{2}{*}{0} & 4952.58 & \multirow{2}{*}{-11} & 17106.7 & \multirow{2}{*}{-1} \\
\hline & & Base & 5014 & & 16939 & & 4486 & & 16683 & & 4972 & & 17686 & & 4481 & & 16950 & \\
\hline & & $36 \%$ & 5136.71 & \multirow{2}{*}{-8} & 18321 & \multirow{2}{*}{-12} & 4995.19 & \multirow{2}{*}{-17} & 18575.8 & \multirow{2}{*}{-15} & 5069.64 & \multirow{2}{*}{-6} & 18914.5 & \multirow{2}{*}{-11} & 5007.6 & 13 & 18278.1 & \multirow{2}{*}{-17} \\
\hline & & Base & 4756 & & 16333 & & 4256 & & 16106 & & 4764 & & 17058 & & 4419 & -13 & 15581 & \\
\hline & & $20 \%$ & 5125.82 & \multirow{2}{*}{-11} & 17166.4 & \multirow{2}{*}{-13} & 4972.23 & \multirow{2}{*}{-20} & 17408.9 & \multirow{2}{*}{-17} & 5065.96 & \multirow{2}{*}{-10} & 17745.8 & \multirow{2}{*}{-10} & 4983.5 & 21 & $\mid$\begin{tabular}{|l|}
17107.2 \\
\end{tabular} & 1 \\
\hline & & Base & 4617 & & 15244 & & 4139 & & 14825 & & 4611 & & 16078 & & 4122 & -21 & 15219 & -12 \\
\hline
\end{tabular}

Table 8 Courtyard 1 with Power density $1.32 \mathrm{~W} / \mathrm{m} 2$ and ac/h 0.6

\begin{tabular}{|c|c|c|c|c|c|c|c|c|c|c|c|c|c|c|c|c|c|c|}
\hline \multicolumn{3}{|c|}{\begin{tabular}{|l|l} 
PD-1.32 W/m2 & ACH-0.6 \\
\end{tabular}} & & & & & & & & & & & & & & & & \\
\hline \multicolumn{3}{|c|}{ Courtyard type -1 (1:1.5) } & North & & & & East & & & & South & & & & West & & & \\
\hline \multicolumn{3}{|c|}{ Year round energy } & Elec(kWh) & Diff. (\%) & Gas(kWh) & Diff. (\%) & Elec(kWh) & Diff. (\%) & Gas(kWh) & Diff. (\%) & Elec(kWh) & Diff. (\%) & Gas(kWh) & Diff. (\%) & Elec(kWh) & Diff. (\%) & Gas(kWh) & Diff. (\%) \\
\hline \multirow{8}{*}{ Glazing } & \multirow{8}{*}{$\begin{array}{c}\text { Dble clr, } \\
6 \mathrm{~mm} / 13 \\
\mathrm{~mm} \text { air }\end{array}$} & $77 \%$ & 4556.18 & \multirow{2}{*}{10} & 15200.5 & \multirow{2}{*}{11} & 4407.18 & \multirow{2}{*}{8} & 15383.4 & \multirow{2}{*}{5} & 4507.27 & \multirow{2}{*}{12} & \begin{tabular}{|l|l|}
15747.3 \\
\end{tabular} & \multirow{2}{*}{17} & 4420.5 & \multirow{2}{*}{7} & 15111.3 & \multirow{2}{*}{10} \\
\hline & & Base & 5072 & & 17114 & & 4778 & & 16183 & & 5114 & & 18925 & & 4736 & & 16836 & \\
\hline & & $57 \%$ & 4572.14 & \multirow{2}{*}{9} & 15210 & \multirow{2}{*}{10} & 4420.71 & \multirow{2}{*}{1} & 15402.1 & \multirow{2}{*}{8} & 4522.38 & \multirow{2}{*}{9} & 15761.3 & \multirow{2}{*}{11} & 4433.79 & \multirow{2}{*}{1} & 15121.6 & \multirow{2}{*}{11} \\
\hline & & Base & 5014 & & 16939 & & 4486 & & 16683 & & 4972 & & 17686 & & 4481 & & 16950 & \\
\hline & & $36 \%$ & 4618.78 & \multirow{2}{*}{3} & 16311 & \multirow{2}{*}{0} & 4475.72 & \multirow{2}{*}{-5} & 16548.5 & \multirow{2}{*}{-3} & 4562.19 & \multirow{2}{*}{4} & 16881.3 & \multirow{2}{*}{1} & 4487.96 & \multirow{2}{*}{-2} & 16254.3 & \multirow{2}{*}{-4} \\
\hline & & Base & 4756 & & 16333 & & 4256 & & 16106 & & 4764 & & 17058 & & 4419 & & 15581 & \\
\hline & & $20 \%$ & 4607.4 & \multirow{2}{*}{0} & 15190.7 & \multirow{2}{*}{0} & 4452.26 & \multirow{2}{*}{-8} & 15415.8 & \multirow{2}{*}{-4} & 4557.99 & \multirow{2}{*}{1} & 15750.9 & 2 & 4463.53 & 8 & 15117.9 & 1 \\
\hline & & Base & 4617 & & 15244 & & 4139 & & 14825 & & 4611 & & 16078 & 2 & 4122 & -8 & 15219 & 1 \\
\hline
\end{tabular}

Table 9 Courtyard 2 with Power density $1.63 \mathrm{~W} / \mathrm{m} 2$ and ac/h 0.74

\begin{tabular}{|c|c|c|c|c|c|c|c|c|c|c|c|c|c|c|c|c|c|c|}
\hline \multirow{2}{*}{\multicolumn{3}{|c|}{\begin{tabular}{|c|c|} 
PD-1.63 W/m2 & ACH-0.74 \\
Courtyard type -2 (1:1)
\end{tabular}}} & & & & & & & & & & & & & & & & \\
\hline & & & North & & & & East & & & & South & & & & West & & & \\
\hline \multicolumn{3}{|c|}{ Year round energy } & Elec(kWh) & Diff. (\%) & Gas(kWh) & Diff. (\%) & Elec(kWh) & Diff. (\%) & Gas(kWh) & Diff. (\%) & Elec(kWh) & Diff. (\%) & Gas(kWh) & Diff. (\%) & Elec(kWh) & Diff. (\%) & Gas(kWh) & Diff. (\%) \\
\hline \multirow{8}{*}{ Glazing } & \multirow{8}{*}{$\begin{array}{c}\text { Dble clr, } \\
6 \mathrm{~mm} / 13 \\
\mathrm{~mm} \text { air }\end{array}$} & $73 \%$ & 4851.59 & \multirow{2}{*}{4} & 17290.4 & \multirow{2}{*}{-1} & 4703.55 & \multirow{2}{*}{-4} & 17554.3 & \multirow{2}{*}{-4} & 4781.38 & \multirow{2}{*}{3} & \begin{tabular}{|l|}
17883.2 \\
\end{tabular} & \multirow{2}{*}{2} & 4717.4 & \multirow{2}{*}{-7} & 17258.9 & \multirow{2}{*}{3} \\
\hline & & Base & 5075 & & 17083 & & 4544 & & 16844 & & 4931 & & 18253 & & 4411 & & 17864 & \\
\hline & & $55 \%$ & 4865.81 & \multirow[b]{2}{*}{-2} & 17291.3 & \multirow[b]{2}{*}{-3} & 4715.91 & \multirow[b]{2}{*}{-8} & 17559.5 & \multirow{2}{*}{-7} & 4796.4 & \multirow{2}{*}{1} & 17883.5 & \multirow{2}{*}{-4} & 4729.39 & \multirow{2}{*}{-8} & 17262.7 & \multirow{2}{*}{-6} \\
\hline & & Base & 4767 & & 16864 & & 4347 & & 16356 & & 4842 & & 17278 & & 4375 & & 16217 & \\
\hline & & $36 \%$ & 4885.99 & \multirow{2}{*}{-3} & 17290.8 & \multirow{2}{*}{-10} & 4734.75 & \multirow{2}{*}{-11} & 17565.5 & \multirow{2}{*}{-17} & 4817.39 & \multirow{2}{*}{-3} & 17887.2 & -7 & 4747.76 & \multirow{2}{*}{-13} & 17266.4 & \multirow{2}{*}{-11} \\
\hline & & Base & 4733 & & 15653 & & 4277 & & 15017 & & 4675 & & 16667 & -1 & 4203 & & 15553 & \\
\hline & & $23 \%$ & 4896.06 & \multirow{2}{*}{-6} & 17308.3 & \multirow{2}{*}{-15} & 4743.98 & \multirow{2}{*}{-16} & 17591.8 & \multirow{2}{*}{-18} & 4828.39 & \multirow{2}{*}{-5} & 17904.4 & \multirow{2}{*}{-12} & 4756.08 & \multirow{2}{*}{-16} & 17288.5 & \\
\hline & & Base & 4611 & & 15008 & & 4106 & & 14903 & & 4586 & & 15978 & & 4100 & & 15308 & -13 \\
\hline
\end{tabular}

Table 10 Courtyard 2 with Power density $1.32 \mathrm{~W} / \mathrm{m} 2$ and ac/h 0.6

\begin{tabular}{|c|c|c|c|c|c|c|c|c|c|c|c|c|c|c|c|c|c|c|}
\hline PD-1.32 V & $\mathrm{V} / \mathrm{m} 2$ & ACH-0.6 & & & & & & & & & & & & & & & & \\
\hline Court & yard type & $2(1: 1)$ & North & & & & East & & & & South & & & & West & & & \\
\hline Yea & $r$ round en & ergy & Elec(kWh) & Diff. (\%) & Gas(kWh) & Diff. (\%) & Elec(kWh) & Diff. (\%) & Gas(kWh) & Diff. (\%) & Elec(kWh) & Diff. (\%) & Gas(kWh) & Diff. (\%) & Elec(kWh) & Diff. (\%) & Gas(kWh) & Diff. (\%) \\
\hline & & $73 \%$ & 4334.39 & 15 & \begin{tabular}{|l|}
15281.1 \\
\end{tabular} & 11 & \begin{tabular}{|l|}
4184.58 \\
\end{tabular} & 0 & 15526.8 & 8 & 4264.05 & 14 & 15851.7 & 13 & 4198.19 & 5 & 15235 & 15 \\
\hline & & Base & 5075 & 15 & 17083 & 11 & 4544 & 8 & 16844 & 8 & 4931 & 14 & 18253 & 13 & 4411 & 5 & 17864 & 15 \\
\hline & & $55 \%$ & 4348.22 & 0 & 15281.4 & 0 & 4197.06 & 3 & 15535 & 5 & 4278.87 & 12 & 15855.2 & 0 & 4210 & 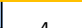 & 15239.6 & 6 \\
\hline Cran & Dble clr, & Base & 4767 & 9 & 16864 & 9 & 4347 & 3 & 16356 & 5 & 4842 & 12 & 17278 & 8 & 4375 & 4 & 16217 & 6 \\
\hline Glazing & $6 \mathrm{~mm} / 13$ & $36 \%$ & 4368.21 & 8 & 15279.5 & 2 & 4215.28 & 1 & 15540.5 & 3 & 4299.5 & 8 & 15855.5 & 5 & 4228.02 & 1 & 15243.5 & 2 \\
\hline & & Base & 4733 & 8 & 15653 & 2 & 4277 & 1 & 15017 & -3 & 4675 & 8 & 16667 & 5 & 4203 & -1 & 15553 & 2 \\
\hline & & $23 \%$ & 4377.68 & 5 & 15295.2 & 2 & 4223.7 & 3 & 15564.7 & 0 & 4309.96 & 6 & 15872.7 & 1 & 4235.77 & 3 & 15266.4 & 0 \\
\hline & & Base & 4611 & 5 & 15008 & -2 & 4106 & -3 & 14903 & -4 & 4586 & 6 & 15978 & 1 & 4100 & -3 & 15308 & 0 \\
\hline
\end{tabular}




\subsection{Daylight analysis methodology}

Most of the daylighting analysis metrics are illumination based. Illumination of light indicates the amount of light falling on the surface, independent of surface type (Cuttle, 2003). Design Builder can analysis daylight in two ways:

Firstly, 'traditional' daylight distribution method, that is static method considered at a given point in time. This method uses radiance engine for daylight modelling (DesignBuilder software guide, 2009).

Secondly, the Climate Based Daylight Modelling (CBDM) that uses realistic sun and sky conditions and predict illumination. To capture the seasonal dynamics of natural daylight, this dynamic metric carries out for a full year at a time step of an hour. For this analysis Design Builder uses Daysim daylighting engine (DesignBuilder software guide, 2009).

The static (Daylight Factor) and dynamic (Spatial Daylight Autonomy) metric can be explained as below.

\section{DF (Daylight factor, Static metric)}

It is the ratio represents the amount of illumination available in indoor relative to present outdoor for same overcast sky. For example, if indoor illuminance is 400 lux, outdoor is 20,000 lux, then DF $=400 / 20,000 \times 100=2$ (Advanced Buildings, 2018). 


\section{sDA (Spatial Daylight Autonomy, Dynamic metric)}

This Spatial Daylight Autonomy is a dynamic daylight metric represents as a percentage of an annual daytime hours (usually $50 \%$ ) that a given point in a space is above specified illumination level. The user is free to set the threshold above which Daylight Autonomy is calculated (Advanced Buildings, 2018; DesignBuilder software guide, 2009).

Vaisman (2015) found that largest courtyard footprint in row house shows better illuminance hours, but there is a small difference on the energy consumption (Vaisman, 2015). In this research more, focus will be given on area covered by target illuminance. So, it will use DF (Daylight factor) and Target illuminance metrics for daylight analysis.

\subsection{Variables and indicators}

The variables and indicators are used in this research is:

Table 11 Variables and indicators

\begin{tabular}{|l|l|l|}
\hline Variable & Indicator & Comment \\
\hline Energy load & $\begin{array}{l}\text { Kilowatt-hour of Energy } \\
\text { consumption by per } \mathrm{m} 2 \text { area }\end{array}$ & $\begin{array}{l}\text { Energy consumtion } \\
\text { represents here mainly for } \\
\text { gas and electricity } \\
\text { consumption }\end{array}$ \\
\hline Daylight condition & $\begin{array}{l}\text { Percentage of area covered by } \\
\text { traget illuminance }\end{array}$ & $\begin{array}{l}\text { Increase area with taget } \\
\text { illuminance shows the } \\
\text { improvement in daylighting }\end{array}$ \\
\hline
\end{tabular}




\subsection{Detailed variables}

To understand the energy performance of atriums compared to courtyard during winter in row houses of Toronto, the following detailed variables are used to analyze the simulation models -

\section{- Courtyard configuration}

- Configuration -1 (Length and width ratio of courtyard is $1: 1.5$ )

- Configuration -2 (Length and width ratio of courtyard is 1:1)

- Courtyard orientation

- Four orientation, i.e. North, East, South and West

- Glazing percentage (WWR - Window to Wall Ratio)

- Configuration -1: WWR -77\%, WWR - 57\%, WWR -36\%, WWR - 20\%

○ Configuration -2: WWR - 73\%, WWR -53\%, WWR - 36\%, WWR - 23\%

- Glazing type

○ Courtyard glazing

- $\mathrm{Dbl} \mathrm{clr,} 6 \mathrm{~mm} / 13 \mathrm{~mm}$ air

- Dbl LoE (e2=.1) Clr 6mm/13mm Air

- Trp Clr 3mm/13mm Air

- $\operatorname{Trp} \operatorname{LoE}(\mathrm{e} 2=e 5=.1) \mathrm{Clr} 3 \mathrm{~mm} / 13 \mathrm{~mm}$ Air

$\circ$ Atrium glazing

- Dbl clr, $6 \mathrm{~mm} / 13 \mathrm{~mm}$ air

- Dbl LoE (e2=.1) Clr 6mm/13mm Air 


\section{Analysis and Result}

\subsection{Base case}

Table 12 Energy consumption of courtyard house for configuration -1

\begin{tabular}{|c|c|c|c|c|c|c|c|c|c|c|c|c|c|c|}
\hline \multirow{2}{*}{\multicolumn{3}{|c|}{\begin{tabular}{|l|} 
Courtyard type -1 (1:1.5) \\
November - April energy \\
\end{tabular}}} & \multicolumn{3}{|c|}{ North } & \multicolumn{3}{|c|}{ East } & \multicolumn{3}{|c|}{ South } & \multicolumn{3}{|c|}{ West } \\
\hline & & & Elec(kWh) & Gas(kWh) & $\mathrm{kWh} / \mathrm{m} 2$ & Elec(kWh) & 1) $\operatorname{Gas}(\mathrm{kWh})$ & $\mathrm{kWh} / \mathrm{m} 2$ & Elec(kWh) & Gas(kWh) & $\mathrm{kWh} / \mathrm{m} 2$ & Elec(kWh) & Gas(kWh) & $\mathrm{kWh} / \mathrm{m} 2$ \\
\hline \multirow{4}{*}{ Glazing } & \multirow{4}{*}{$\begin{array}{c}\text { Courtyard } \\
\text { Dble clr, } \\
6 \mathrm{~mm} / 13 \mathrm{~mm} \\
\text { air }\end{array}$} & $77 \%$ & 2297.31 & 17755.55 & \begin{tabular}{|l|}
123.45 \\
\end{tabular} & 2236.55 & 16499.58 & 115.35 & 2328.33 & 16242.13 & 114.33 & 2255.05 & 16768.04 & 117.11 \\
\hline & & $57 \%$ & 2273.78 & 17136.29 & 119.49 & 2207.76 & 16097.84 & 112.69 & 2292.3 & 16062.7 & 113 & 2215.18 & 16359.63 & 114.35 \\
\hline & & $36 \%$ & 2227.91 & 18019.28 & 124.65 & 2161.37 & 17937.77 & 123.74 & 2228.35 & 17198.3 & 119.6 & 2182.55 & 16880.7 & 117.36 \\
\hline & & $20 \%$ & 2228.11 & 16043.38 & 112.48 & 2146.85 & 15582.01 & 109.14 & 2226.23 & 16091.68 & 112.77 & 2131.77 & 15843.25 & 110.66 \\
\hline
\end{tabular}

Table 13 Daylighting condition of courtyard house for configuration -1

\begin{tabular}{|c|c|c|c|c|c|c|c|}
\hline \multicolumn{4}{|c|}{ Courtyard type -1 (1:1.5) } & North & East & South & West \\
\hline \multicolumn{3}{|c|}{ November - April energy } & Daylight & $\%$ of area above threshold & $\%$ of area above threshold & $\%$ of area above threshold & $\%$ of area above threshold \\
\hline \multirow{4}{*}{ Glazing } & \multirow{4}{*}{$\begin{array}{c}\text { Courtyard } \\
\text { Dble clr, } \\
6 \mathrm{~mm} / 13 \mathrm{~m} \\
\text { m air }\end{array}$} & $77 \%$ & Court & 99.694 & 99.898 & 99.796 & 100 \\
\hline & & $57 \%$ & Court & 99.694 & 99.796 & 99.694 & 99.796 \\
\hline & & $36 \%$ & Court & 85.903 & 86.107 & 86.72 & 86.005 \\
\hline & & $20 \%$ & Court & 73.338 & 71.806 & 71.397 & 72.113 \\
\hline
\end{tabular}

Table 14 Energy consumption of courtyard house for configuration -2

\begin{tabular}{|c|c|c|c|c|c|c|c|c|c|c|c|c|c|c|}
\hline \multicolumn{3}{|c|}{ Courtyard type -2 (1:1) } & \multicolumn{3}{|c|}{ North } & \multicolumn{3}{|c|}{ East } & \multicolumn{3}{|c|}{ South } & \multicolumn{3}{|c|}{ West } \\
\hline Novembe & - April energy & & Elec(kWh) & Gas(kWh) & $\mathrm{kWh} / \mathrm{m} 2$ & Elec(kWh) & Gas(kWh) & $\mathrm{kWh} / \mathrm{m} 2$ & Elec(kWh) & Gas(kWh) & $\mathrm{kWh} / \mathrm{m} 2$ & Elec(kWh) & ) Gas(kWh) & $\mathrm{kWh} / \mathrm{m} 2$ \\
\hline \multirow{4}{*}{ Glazing } & \multirow{4}{*}{$\begin{array}{l}\text { Courtyard } \\
\text { Dble clr, } \\
6 \mathrm{~mm} / 13 \mathrm{~mm} \\
\text { air }\end{array}$} & $73 \%$ & 2329.95 & 17536.52 & 119.01 & 2256.16 & 16660.25 & 113.32 & 2350.38 & 16844.73 & 114.99 & 2249.67 & 16924.18 & 114.86 \\
\hline & & $55 \%$ & 2317.58 & 17046.87 & 116 & 2238.53 & 16287.28 & 110.98 & 2330.3 & 16588.68 & 113.33 & 2230.73 & 16546.82 & 112.48 \\
\hline & & $36 \%$ & 2290.8 & 16648.45 & 113.45 & 2212.08 & 16135.82 & 109.91 & 2295.93 & 16623.26 & 113.33 & 2199.2 & 16385.62 & 111.33 \\
\hline & & $23 \%$ & 2276.58 & 16251.62 & 110.99 & 2197.92 & 15880.4 & 108.3 & 2275.88 & 16453.34 & 112.19 & 2182.41 & 16125.17 & 109.67 \\
\hline
\end{tabular}

Table 15 Daylighting condition of courtyard house for configuration -2

\begin{tabular}{|c|c|c|c|c|c|c|c|}
\hline \multicolumn{4}{|c|}{ Courtyard type $-2(1: 1)$} & North & East & South & West \\
\hline \multicolumn{3}{|c|}{ November - April energy } & Daylight & $\%$ of area above threshold & $\%$ of area above threshold & $\%$ of area above threshold & $\%$ of area above threshold \\
\hline \multirow{4}{*}{ Glazing } & \multirow{4}{*}{\begin{tabular}{|c|} 
Courtyard \\
Dble clr, \\
$6 \mathrm{~mm} / 13 \mathrm{~m}$ \\
$\mathrm{~m}$ air \\
\end{tabular}} & $73 \%$ & Court & 99.697 & 99.092 & 99.193 & 98.991 \\
\hline & & $55 \%$ & Court & 98.184 & 98.083 & 98.789 & 98.083 \\
\hline & & $36 \%$ & Court & 78.914 & 78.51 & 78.914 & 78.813 \\
\hline & & $23 \%$ & Court & 63.579 & 63.881 & 64.487 & 62.368 \\
\hline
\end{tabular}

Base cases are developed for this research considering the energy consumption by row

house with courtyard during winter (peak heating period, i.e. November to April).

Daylighting condition during this period also plotted, where daylighting condition is

expressed by the percentage of floor area covered by target illuminance. Base cases 
are elaborated for different orientations and for different WWRs in both courtyard

configurations. For all these cases glazing typology - Double clear, $6 \mathrm{~mm} / 13 \mathrm{~mm}$ air is

considered for all windows.

\subsection{Energy performance of atrium house compared to courtyard house}

\section{during winter}

\subsubsection{Reduction of energy consumption by atrium house during winter}

Table 16 Reduction in energy consumption by atrium house compared to courtyard house for configuration -1

\begin{tabular}{|c|c|c|c|c|c|c|c|c|c|c|c|c|c|c|c|c|c|c|c|}
\hline \multicolumn{4}{|c|}{ Courtyard type -1 (1:1.5) } & \multicolumn{4}{|c|}{ North } & \multicolumn{4}{|c|}{ East } & \multicolumn{4}{|c|}{ South } & \multicolumn{4}{|c|}{ West } \\
\hline \multicolumn{3}{|c|}{ November - April energy } & Option & Elec(kWh) & 1) Gas(kWh) & 1) $\mathrm{kWh} / \mathrm{m} 2$ & Decrease & Elec(kWh) & 1) Gas(kWh) & $\mathrm{kWh} / \mathrm{m} 2$ & 2 Decrease & ElecklkWh & 1) Gas(kWh) & $\mathrm{kWh} / \mathrm{m} 2$ & Decrease & $\% \operatorname{Elec}(\mathrm{kWh})$ & 1) Gas(kWh) & $\mathrm{kWh} / \mathrm{m} 2$ & Decrease\% \\
\hline \multirow{8}{*}{ Glazing } & \multirow{8}{*}{$\begin{array}{l}\text { Courtyard Dble clr, } \\
6 \mathrm{~mm} / 13 \mathrm{~mm} \text { air } \\
\text { and sky light Dble } \\
\mathrm{clr}, 6 \mathrm{~mm} / 13 \mathrm{~mm} \\
\text { air }\end{array}$} & \multirow{2}{*}{$77 \%$} & Court & 2297.31 & 17755.55 & 123.45 & -20 & 2236.55 & 16499.58 & 115.35 & -15 & 2328.33 & 16242.13 & 114.33 & -11 & 2255.05 & 16768.04 & 117.11 & -15 \\
\hline & & & Atrium & 2325.25 & 13802.45 & 99.29 & & 2327.27 & 13685.44 & 98.58 & & 2326.73 & 14168.6 & 101.55 & & 2327.91 & 13755.19 & 99.01 & \\
\hline & & \multirow{2}{*}{$57 \%$} & Court & 2273.78 & 17136.29 & 119.49 & -17 & 2207.76 & 16097.84 & 112.69 & -12 & 2292.3 & 16062.7 & 113 & -10 & 2215.18 & 16359.63 & 114.35 & -13 \\
\hline & & & Atrium & 2334.44 & 13825.06 & \begin{tabular}{|l|l|}
69.48 \\
\end{tabular} & & 2336.55 & 13710.53 & 98.79 & & 2336.17 & 14188.14 & 101.73 & & 2337.37 & 13779.45 & 99.22 & \\
\hline & & \multirow{2}{*}{$36 \%$} & Court & 2227.91 & 18019.28 & 124.65 & -15 & 2161.37 & 17937.77 & 123.74 & -15 & 2228.35 & 17198.3 & 119.6 & -10 & 2182.55 & 16880.7 & 117.36 & -10 \\
\hline & & & Atrium & 2360.85 & 14844.64 & 105.92 & & 2363.77 & 14758.44 & 105.41 & & 2363.04 & 15214.04 & 108.21 & & 2364.73 & 14828.4 & 105.85 & \\
\hline & & \multirow{2}{*}{$20 \%$} & Court & 2228.11 & 16043.38 & \begin{tabular}{|l|l}
112.48 \\
\end{tabular} & -11 & 2146.85 & 15582.01 & \begin{tabular}{|l|l|}
109.14 \\
\end{tabular} & -9 & 2226.23 & 16091.68 & 112.77 & -9 & 2131.77 & 15843.25 & 110.66 & -10 \\
\hline & & & Atrium & 2350.37 & 13869.59 & 99.86 & & 2353.31 & 13761.46 & 99.21 & & 2352.73 & 14225.05 & 102.06 & & 2354.31 & 13829.16 & 99.63 & \\
\hline
\end{tabular}

Table 17 Reduction in energy consumption by atrium house compared to courtyard house for configuration -2

\begin{tabular}{|c|c|c|c|c|c|c|c|c|c|c|c|c|c|c|c|c|c|c|c|}
\hline \multicolumn{4}{|c|}{ Courtyard type -2 (1:1) } & \multicolumn{4}{|c|}{ North } & \multicolumn{4}{|c|}{ East } & \multicolumn{4}{|c|}{ South } & \multicolumn{4}{|c|}{ West } \\
\hline \multicolumn{3}{|c|}{ November - April energy } & Option & Elec(kWh) & 1) $\operatorname{Gas}(\mathrm{kWh})$ & 1) $\mathrm{kWh} / \mathrm{m} 2$ & Decrease & Elec(kWh) & ) Gas(kWh & $\mathrm{kWh} / \mathrm{m} 2$ & 2 Decrease & Elecc|kWh & 1) Gas(kWh) & $\mathrm{kWh} / \mathrm{m} 2$ & Decrease & GElec(kWh & 1) Gas(kWh) & $\mathrm{kWh} / \mathrm{m} 2$ & Decrease\% \\
\hline \multirow{8}{*}{ Glazing } & \multirow{8}{*}{$\begin{array}{l}\text { Courtyard Dble clr, } \\
6 \mathrm{~mm} / 13 \mathrm{~mm} \text { air } \\
\text { and sky light Dble } \\
\mathrm{clr}, 6 \mathrm{~mm} / 13 \mathrm{~mm} \text { air }\end{array}$} & \multirow{2}{*}{$73 \%$} & Court & 2329.95 & 17536.52 & 2119.01 & -20 & 2256.16 & 16660.25 & 113.32 & -17 & 2350.38 & 16844.73 & 114.99 & -16 & 2249.67 & 16924.18 & 114.86 & -18 \\
\hline & & & Atrium & 2252.32 & 13588.21 & 94.89 & & 2253.76 & 13471.27 & 94.2 & & 2253.83 & 13963.13 & 97.15 & & 2254.74 & 13543.02 & 94.63 & \\
\hline & & \multirow{2}{*}{$55 \%$} & Court & 2317.58 & 17046.87 & 116 & -18 & 2238.53 & 16287.28 & 110.98 & -15 & 2330.3 & 16588.68 & 113.33 & -14 & 2230.73 & 16546.82 & 112.48 & -16 \\
\hline & & & Atrium & 2257.87 & 13548.94 & 94.69 & & 2259.39 & 13434.53 & 94.01 & & 2259.57 & 13919.41 & 96.92 & & 2260.47 & 13505.14 & 94.44 & \\
\hline & & \multirow{2}{*}{$36 \%$} & Court & 2290.8 & 16648.45 & 113.45 & -16 & 2212.08 & 16135.82 & 109.91 & -14 & 2295.93 & 16623.26 & 113.33 & -14 & 2199.2 & 16385.62 & 111.33 & -15 \\
\hline & & & Atrium & 2263.82 & 13576.67 & $\begin{array}{l}74.89 \\
\end{array}$ & & 2265.79 & 13465.9 & 94.24 & & 2265.8 & 13942.92 & 97.1 & & 2267.02 & 13535.58 & 94.66 & \\
\hline & & \multirow{2}{*}{$23 \%$} & Court & 2276.58 & 16251.62 & 2110.99 & -15 & 2197.92 & 15880.4 & 108.3 & -13 & 2275.88 & 16453.34 & 112.19 & -14 & 2182.41 & 16125.17 & 109.67 & -14 \\
\hline & & & Atrium & 2270.23 & 13543.06 & \begin{tabular}{|l|l|}
694.73 \\
\end{tabular} & & 22272.57 & 13435.41 & 94.1 & & 2272.45 & 13904.96 & 96.91 & & 2273.79 & 13504.35 & 94.52 & \\
\hline
\end{tabular}


Table 18 Improvement in energy consumption by atrium house compared to courtyard house

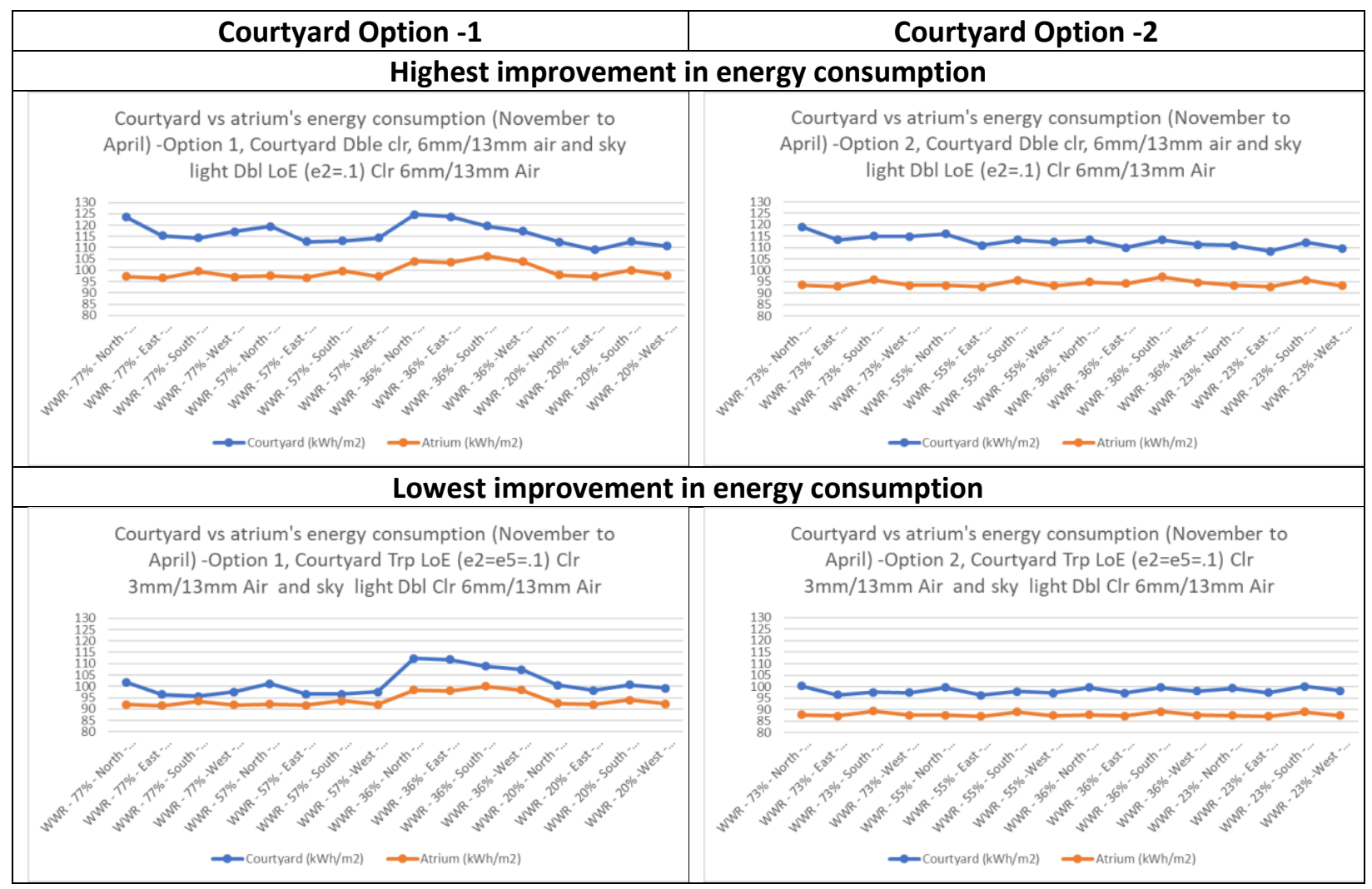

To understand the impact of atrium in row houses in terms of energy consumption during winter, total 32 options are analyzed. Base cases are simulated with glazing typology Double clear $-6 \mathrm{~mm} / 13 \mathrm{~mm}$ air for all windows. Keeping this glazing same for windows in courtyard, when the atrium option is simulated with the same glazing typology for its skylight, that means when windows' and atriums' glazing both are Double clear $6 \mathrm{~mm} / 13 \mathrm{~mm}$ air, highest reduction in energy consumption is found in North oriented option with 77\% WWR for configuration -1 and in North oriented option with $73 \%$ WWR for configuration - 2. In the base cases these options show higher energy consumptions, but in the atrium option they are showing highest energy reduction in consumption. In all cases, both atrium configurations (i.e. 1:1.5 and 1:1) show reduction 
in energy consumption. For the bigger atrium configuration (1:1.5), this improvement ranges from $2 \%-21 \%$ for different options. For the other configuration (configuration-2, $1: 1)$, the improvement ranges from $8 \%-21 \%$. It shows a wide range in energy reduction due to different combination of glazing typology of courtyard and skylight. When we use triple low e glass for courtyard windows and double clear glazing for atrium skylight, it shows lowest reduction in energy consumption by atrium house. But when the scenario is opposite, that means when we use double low e glazing for skylight and double clear glass for courtyard windows, the energy reduction by atrium option is highest, that means reduction is $21 \%$. For both cases reductions are bigger for larger window to wall ratios. So, when the air is modified inside atrium, we can also get maximum reduction in energy consumption by bigger windows. All the comparisons also show that configuration - 2 saves more energy than configuration -1 for atrium house compared to courtyard house during winter. In table -18 two representative graphs are presented and other graphs are attached in appendix. 


\subsubsection{Energy performance of atrium house with different WWR}

Table 19 Energy consumption of atrium house with different Window to Wall Ratio (WWR)

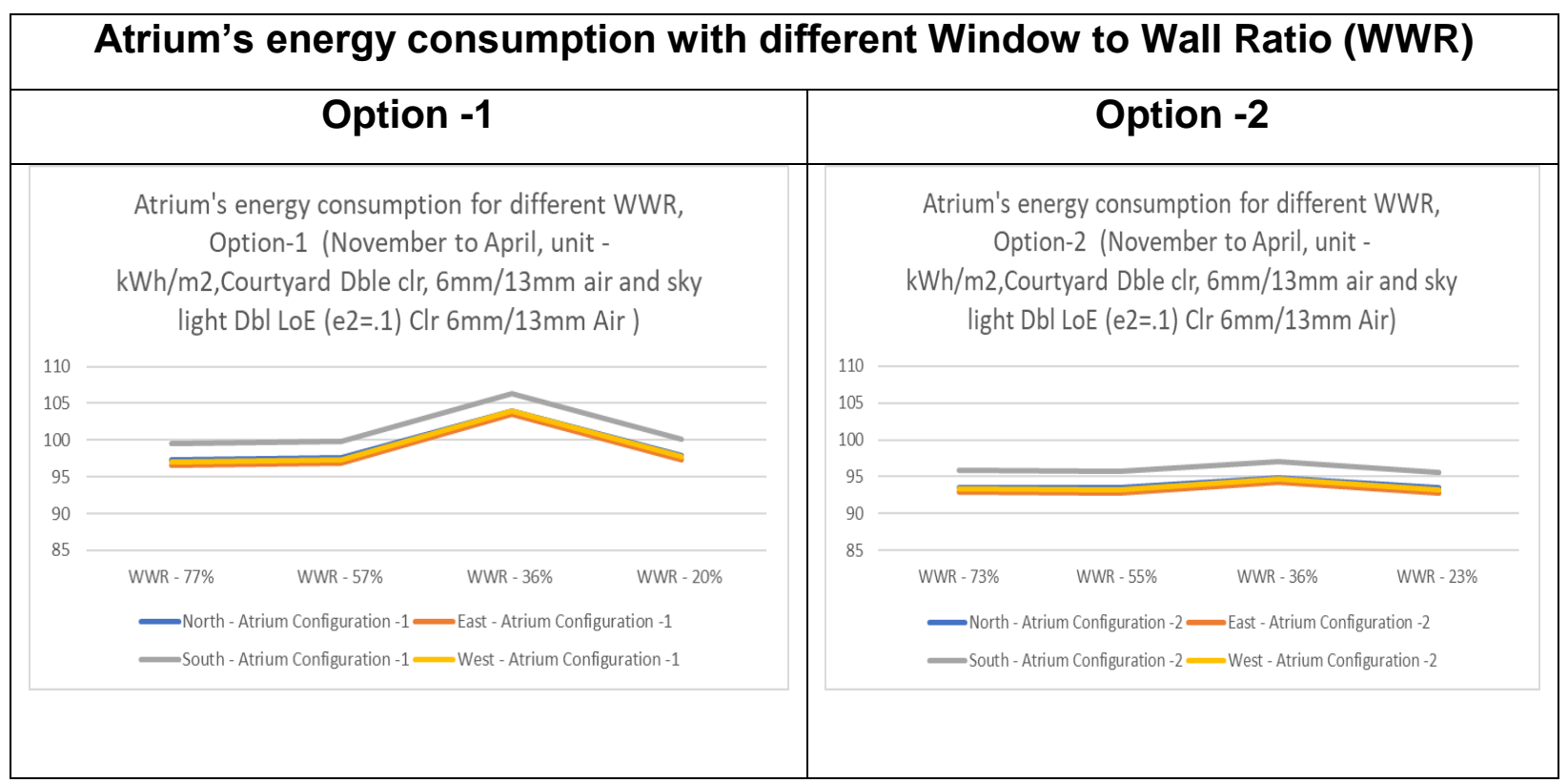

This analysis compares the results of different atrium options among themselves (full result is in appendix). Window to Wall Ratios have significant impact on atrium's energy consumption. For both atrium configurations, house with lower WWRs show higher energy consumption than the houses with higher WWRs. In configuration - 1, all the options with $36 \%$ WWR is critical for energy consumption by atrium house. Due to solar heat gain during daytime, air inside the atrium is heated. With larger WWRs house can be benefitted from this warm air by reducing heating energy load. This continues up to $36 \%$ of WWR. At this point the effect of larger WWR is reduced and energy consumption become higher for $36 \%$ WWR. After that energy consumption by houses effected by the concept of reduced heat loss by reduced WWRs. So, for the lowest WWR we find the reduction in energy consumption for less heat loss by window. In 
configuration - 2, options with $36 \%$ WWRs also shows a little increase in energy consumption than other WWRs, but it is not prominent like configuration -1 . This analysis also shows that atrium options for configuration - 2 consumes less energy than atrium options for configuration 1 .

\subsubsection{Energy performance of atrium house with different orientation}

Table 20 Energy consumption of atrium house with different orientation

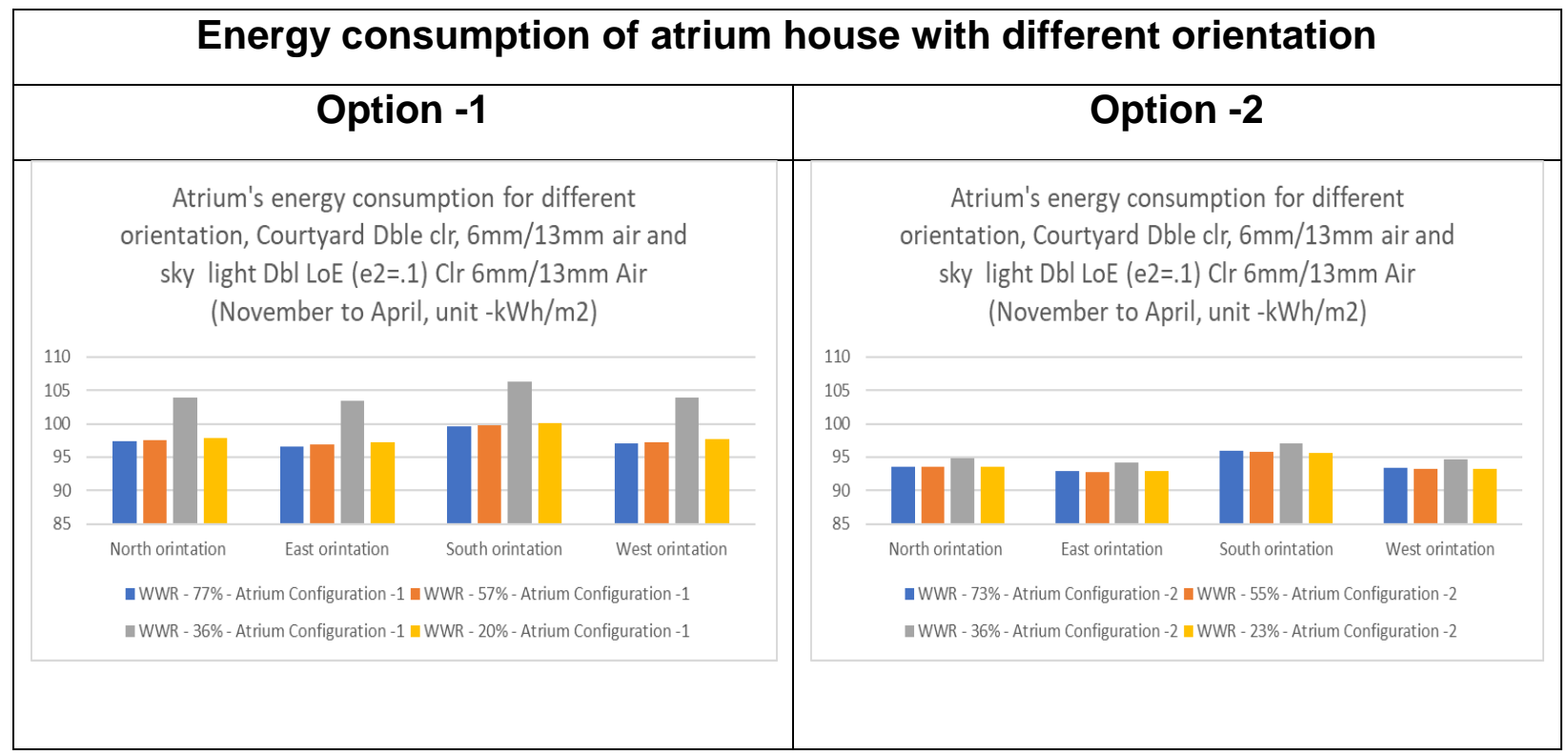

While energy performance of different atrium options is analyzed, it is found that for almost all cases - south oriented atriums show more energy consumption than rest of the three orientations. Specifically, south oriented atrium with $36 \%$ WWR consumes more energy. The reason can be explained from sun path and condition of row house unit. Solar heat gain inside the atrium can reduce heating energy consumption of house during winter. Winter sun path line (runs from east to west) lowers towards south. So, south oriented atrium is supposed to gain solar heat from lateral direction. Since this study house is one of the middle units, it can not be benefitted from south orientation. 


\subsubsection{Energy performance of atrium house with different glazing types}

For this analysis, window's glazing was kept unchanged against different glazing typology of skylight. This analysis can be used as guideline for designing atrium for winter and can be interpreted in different way. For example, if a courtyard has $77 \%$ WWR, courtyard's glazing is double clear, and skylight's glazing is double low e, then from graph it can be said that, in this scenario east orientated atrium can give better result in reduction in energy consumption. Again, if the courtyard's glazing and orientation is fixed, then from graph it can be determined that which glazing typology for sky light will be more appropriate to reduce energy consumption of the house. 
Table 21 Atrium's energy consumption for different glazing types

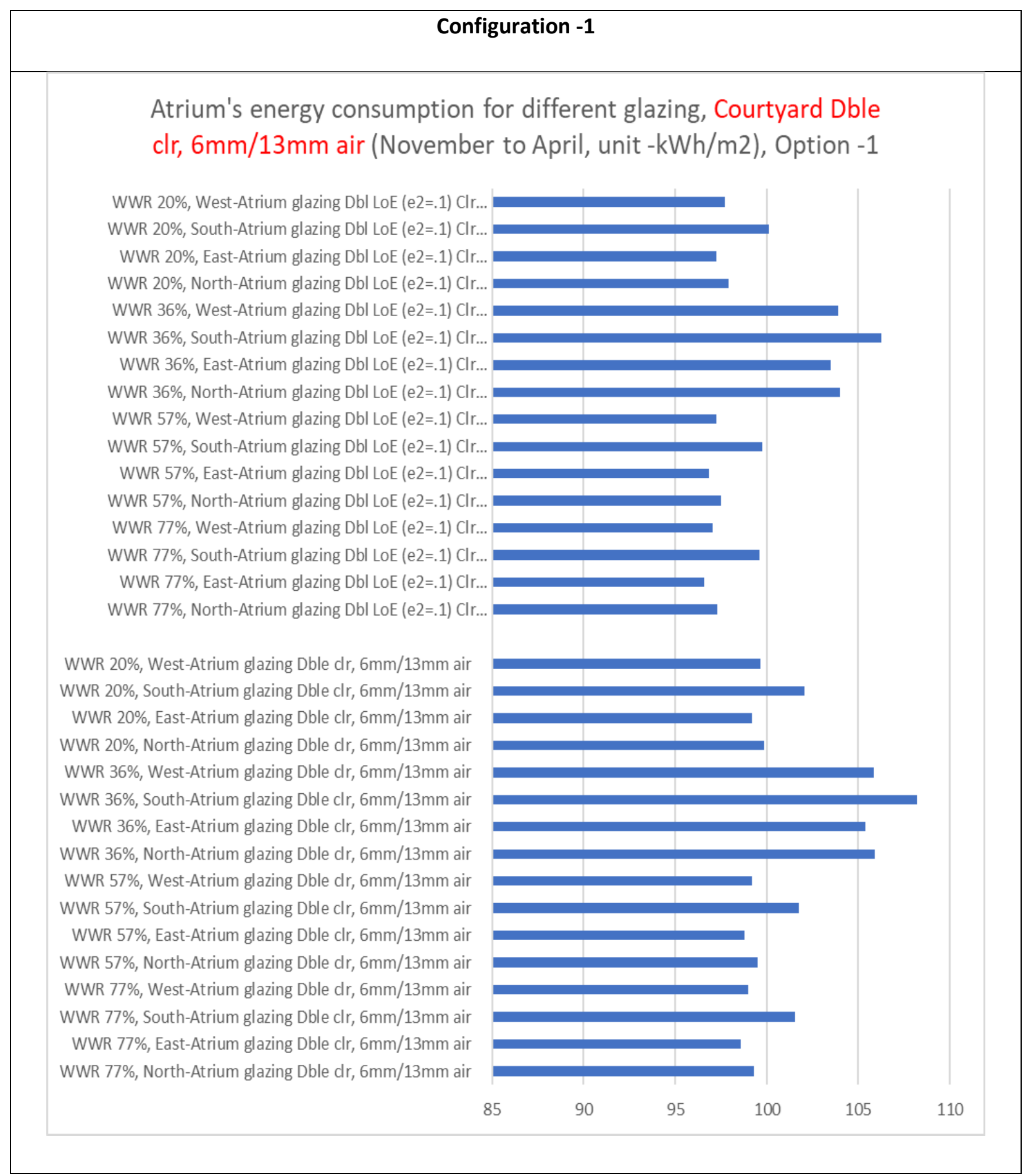




\section{Configuration -2}

\section{Atrium's energy consumption for different glazing, Courtyard Dble $\mathrm{clr}, 6 \mathrm{~mm} / 13 \mathrm{~mm}$ air (November to April, unit -kWh/m2), Option -2}

WWR 23\%, West-Atrium glazing Dbl LoE (e2=.1) Clr... WWR 23\%, South-Atrium glazing Dbl LoE (e2=.1) Clr...

WWR 23\%, East-Atrium glazing Dbl LoE (e2=.1) Clr... WWR 23\%, North-Atrium glazing Dbl LoE (e2=.1) Clr... WWR 36\%, West-Atrium glazing Dbl LoE (e2=.1) Clr... WWR 36\%, South-Atrium glazing Dbl LoE (e2=.1) Clr... WWR 36\%, East-Atrium glazing Dbl LoE (e2=.1) Clr.. WWR 36\%, North-Atrium glazing Dbl LoE (e2=.1) Cr. WWR 55\%, West-Atrium glazing Dbl LoE (e2=.1) WWR 55\%, South-Atrium glazing Dbl LoE (e2=.1)

WWR 55\%, East-Atrium glazing Dbl LoE (e2=.1) WWR 55\%, North-Atrium glazing Dbl LoE (e2=.1) WWR 73\%, West-Atrium glazing Dbl LoE (e2=.1) WWR 73\%, South-Atrium glazing Dbl LoE (e2=.1)

WWR 73\%, East-Atrium glazing Dbl LoE (e2=.1) Cr. WWR 73\%, North-Atrium glazing Dbl LoE (e2=.1) Cr.

WWR $23 \%$, West-Atrium glazing Dble $\mathrm{cr}, 6 \mathrm{~mm} / 13 \mathrm{~mm}$ air WWR 23\%, South-Atrium glazing Dble clr, $6 \mathrm{~mm} / 13 \mathrm{~mm}$ air WWR 23\%, East-Atrium glazing Dble clr, $6 \mathrm{~mm} / 13 \mathrm{~mm}$ air WWR 23\%, North-Atrium glazing Dble clr, $6 \mathrm{~mm} / 13 \mathrm{~mm}$ air WWR $36 \%$, West-Atrium glazing Dble clr, $6 \mathrm{~mm} / 13 \mathrm{~mm}$ air WWR 36\%, South-Atrium glazing Dble clr, $6 \mathrm{~mm} / 13 \mathrm{~mm}$ air WWR $36 \%$, East-Atrium glazing Dble clr, $6 \mathrm{~mm} / 13 \mathrm{~mm}$ air WWR 36\%, North-Atrium glazing Dble $\mathrm{cr}, 6 \mathrm{~mm} / 13 \mathrm{~mm}$ air WWR 55\%, West-Atrium glazing Dble $\mathrm{dr}, 6 \mathrm{~mm} / 13 \mathrm{~mm}$ air WWR 55\%, South-Atrium glazing Dble clr, $6 \mathrm{~mm} / 13 \mathrm{~mm}$ air WWR 55\%, East-Atrium glazing Dble clr, $6 \mathrm{~mm} / 13 \mathrm{~mm}$ air WWR 55\%, North-Atrium glazing Dble dr, $6 \mathrm{~mm} / 13 \mathrm{~mm}$ air WWR $73 \%$, West-Atrium glazing Dble $\mathrm{dr}, 6 \mathrm{~mm} / 13 \mathrm{~mm}$ air WWR 73\%, South-Atrium glazing Dble clr, $6 \mathrm{~mm} / 13 \mathrm{~mm}$ air WWR 73\%, East-Atrium glazing Dble clr, $6 \mathrm{~mm} / 13 \mathrm{~mm}$ air WWR $73 \%$, North-Atrium glazing Dble $\mathrm{dr}, 6 \mathrm{~mm} / 13 \mathrm{~mm}$ air

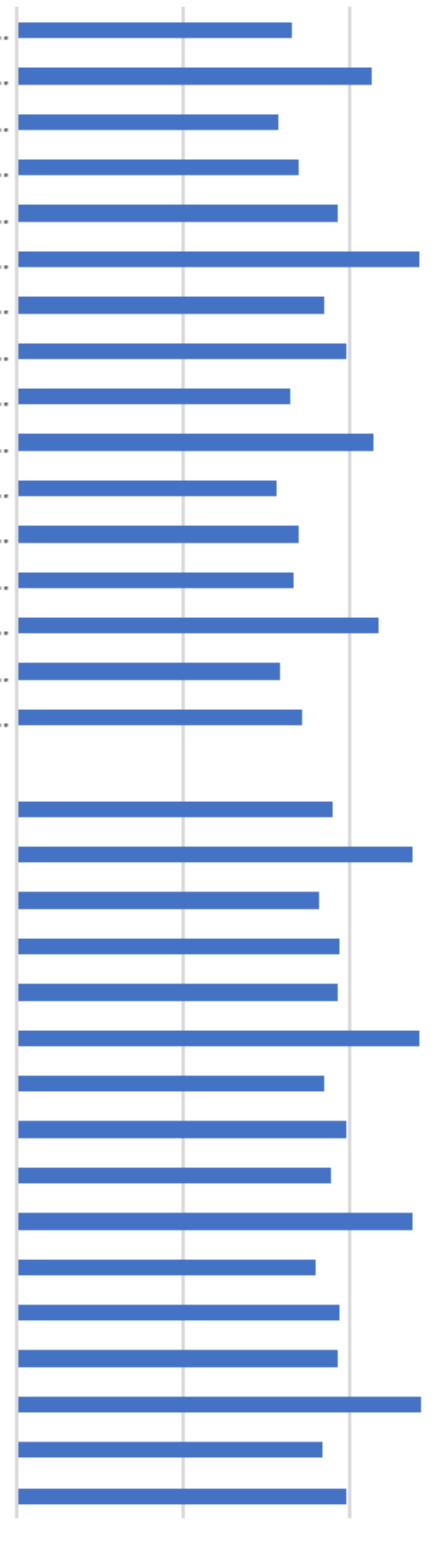




\subsection{Daylight performance of atrium house}

Daylight performance of different atrium options compared with courtyards is analyzed.

In both configurations, percentages of floor area covered by illuminance above

threshold level are reduced in all atrium options.

Table 22 Daylight performance of configuration - 1

\begin{tabular}{|c|c|c|c|c|c|c|c|c|c|c|c|}
\hline \multicolumn{4}{|c|}{ Courtyard type -1 (1:1.5) } & \multicolumn{2}{|l|}{ North } & \multicolumn{2}{|l|}{ East } & \multicolumn{2}{|l|}{ South } & \multicolumn{2}{|l|}{ West } \\
\hline \multicolumn{3}{|c|}{ November - April energy } & Daylight & $\%$ of area above threshold & Decrease \% & $\%$ of area above threshold & Decrease \% & $\%$ of area above threshold & Decrease \% & $\%$ of area above threshold & Decrease \% \\
\hline \multirow{8}{*}{ Glazing } & \multirow{8}{*}{$\begin{array}{l}\text { Courtyard Dble clr, } \\
6 \mathrm{~mm} / 13 \mathrm{~mm} \text { air } \\
\text { and sky light Dble } \\
\mathrm{clr}, 6 \mathrm{~mm} / 13 \mathrm{~mm} \text { air }\end{array}$} & \multirow{2}{*}{$77 \%$} & Court & 99.694 & \begin{tabular}{|l|}
-0.6 \\
\end{tabular} & 99.796 & -0.3 & 99.898 & \begin{tabular}{|l|}
-0.8 \\
\end{tabular} & 99.898 & -0.7 \\
\hline & & & Atrium & 99.081 & & 99.489 & & 99.081 & & 99.183 & \\
\hline & & \multirow{2}{*}{$57 \%$} & Court & 99.796 & -1.1 & 99.898 & -1.2 & 99.694 & -0.6 & 99.796 & -1.0 \\
\hline & & & Atrium & 98.672 & & 98.672 & & 99.081 & & 98.774 & \\
\hline & & \multirow{2}{*}{$36 \%$} & Court & 86.005 & -5.9 & 86.209 & -6.2 & 86.107 & -5.9 & 86.618 & -7.8 \\
\hline & & & Atrium & 80.898 & & 80.898 & & 81 & & 79.876 & \\
\hline & & \multirow{2}{*}{$20 \%$} & Court & 72.215 & -6.2 & 72.419 & -5.8 & 72.113 & -7.2 & 72.93 & -7.0 \\
\hline & & & Atrium & 67.72 & & 68.231 & & 66.903 & & 67.822 & \\
\hline
\end{tabular}

Table 23 Daylight performance of configuration - 2

\begin{tabular}{|c|c|c|c|c|c|c|c|c|c|c|c|}
\hline \multicolumn{4}{|c|}{ Courtyard type -2 (1:1.) } & \multicolumn{2}{|l|}{ North } & \multicolumn{2}{|l|}{ East } & \multicolumn{2}{|l|}{ South } & \multicolumn{2}{|l|}{ West } \\
\hline Novembe & er - April energy & & Daylight & $\%$ of area above threshold & Decrease \% & $\%$ of area above threshold & Decrease \% & $\%$ of area above threshold & Decrease \% & $\%$ of area above threshold & Decrease \% \\
\hline \multirow{8}{*}{ Glazing } & \multirow{8}{*}{$\begin{array}{c}\text { Courtyard Dble clr, } \\
6 \mathrm{~mm} / 13 \mathrm{~mm} \text { air } \\
\text { and sky light Dble } \\
\mathrm{clr}, 6 \mathrm{~mm} / 13 \mathrm{~mm} \text { air }\end{array}$} & \multirow{2}{*}{$73 \%$} & Court & 99.395 & \begin{tabular}{|l|}
-7.2 \\
\end{tabular} & 99.294 & \begin{tabular}{|l|}
-6.3 \\
\end{tabular} & 99.294 & \begin{tabular}{|l|}
-8.5 \\
\end{tabular} & 99.395 & \begin{tabular}{|l|}
-7.7 \\
\end{tabular} \\
\hline & & & Atrium & 92.231 & & 93.039 & & 90.819 & & 91.727 & \\
\hline & & \multirow{2}{*}{$55 \%$} & Court & 99.092 & -7.5 & 97.982 & -7.3 & 98.487 & -7.3 & 98.487 & -9.0 \\
\hline & & & Atrium & 91.626 & & 90.819 & & 91.323 & & 89.608 & \\
\hline & & \multirow{2}{*}{$36 \%$} & Court & 77.098 & -7.9 & 79.419 & -10.4 & 77.502 & -8.9 & 78.006 & -10.9 \\
\hline & & & Atrium & 71.045 & & 71.146 & & 70.641 & & 69.531 & \\
\hline & & \multirow{2}{*}{$23 \%$} & Court & 64.386 & -6.9 & 63.074 & -6.1 & 64.588 & -6.7 & 63.276 & -8.1 \\
\hline & & & Atrium & 59.947 & & 59.241 & & 60.249 & & 58.131 & \\
\hline
\end{tabular}

Table 24 Daylighting condition in configuration 1 \& 2

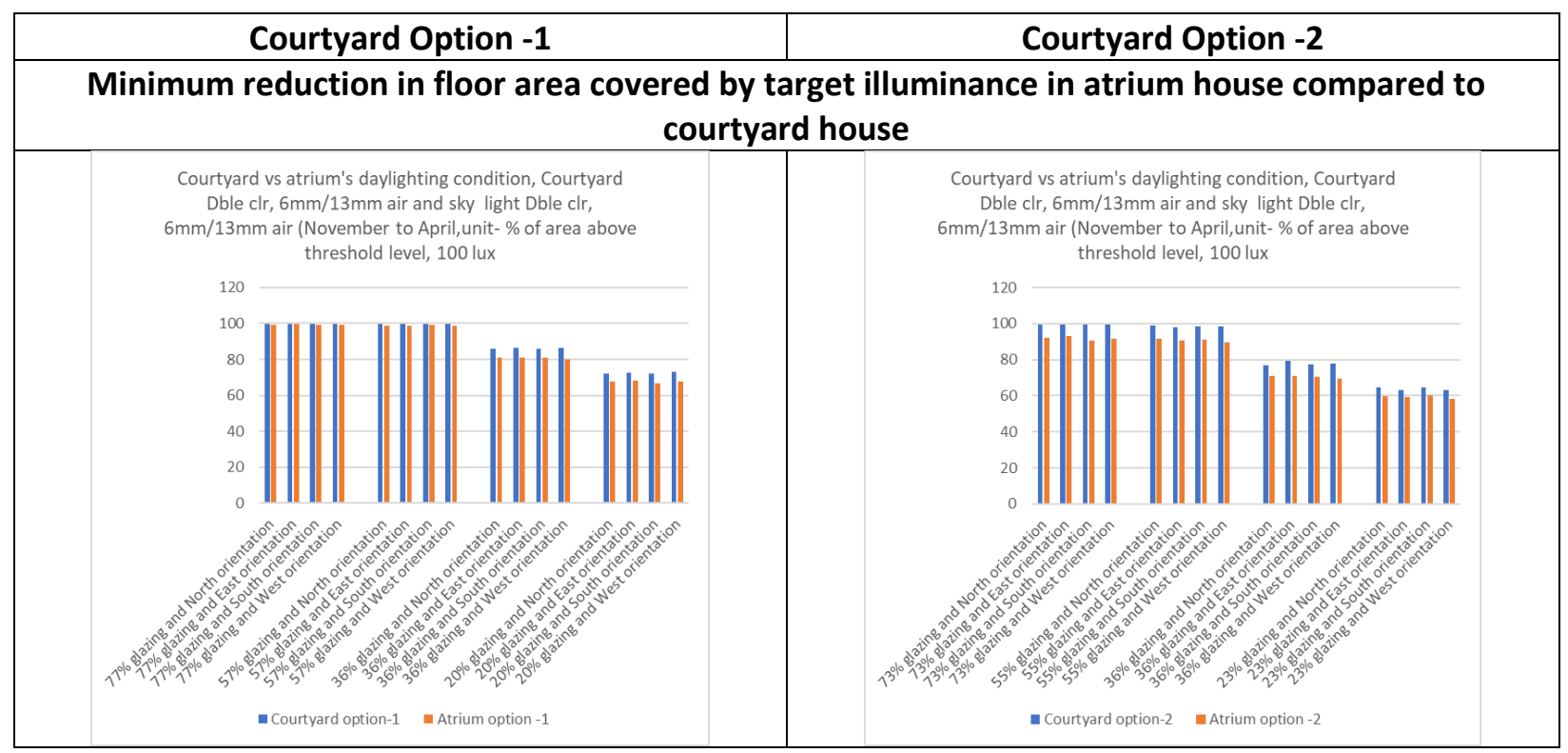




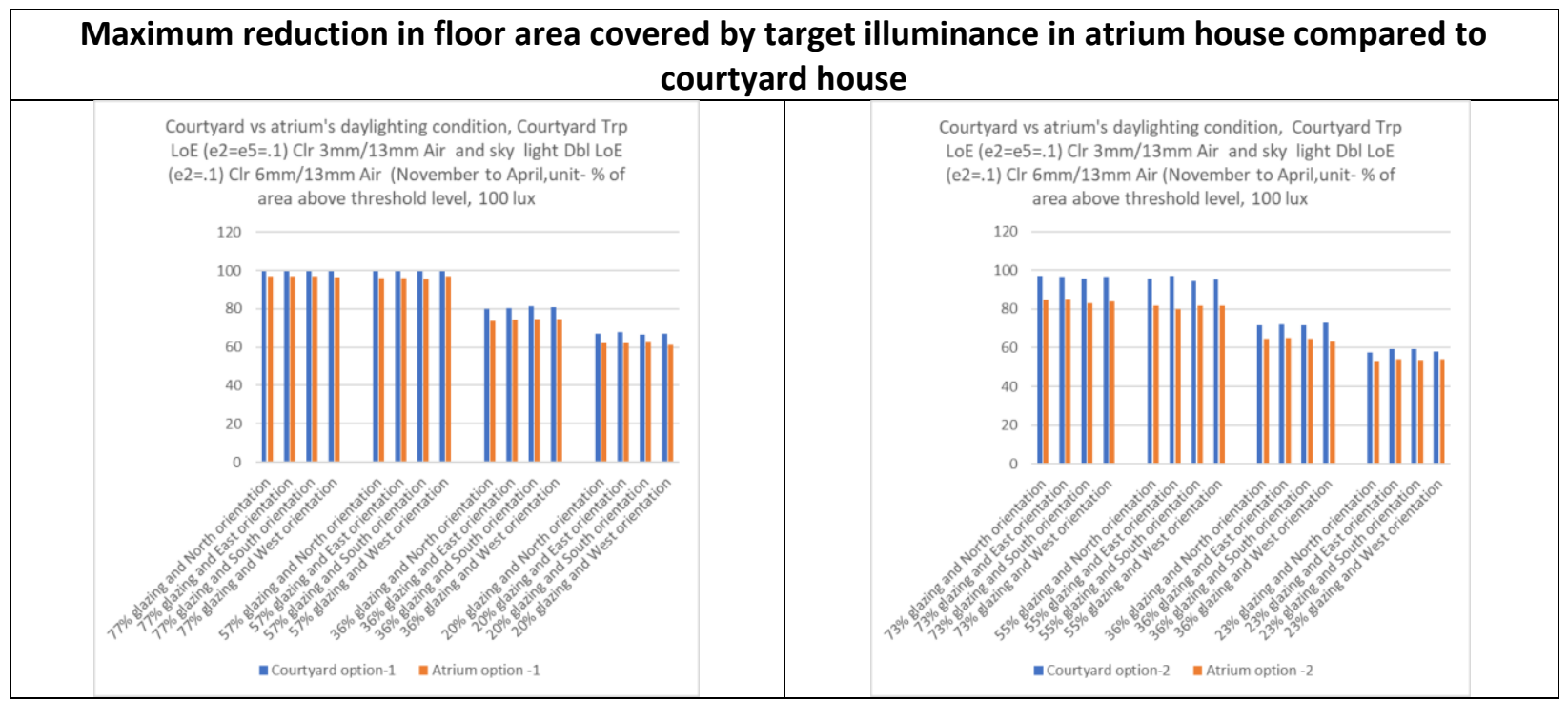

For configuration-1, this reduction is in between, $0.3 \%-23 \%$. In this configuration, this reduction is higher for lower WWRs, i.e. for WWR $-23 \%$ to $W W R-36 \%$.

For the configuration - 2, reduction of floor area covered by target illuminance is ranges from $6 \%-17.3 \%$. Although, for configuration -1 , there is a wide range for daylight reduction, but the overall reduction is high for configuration -2 . This configuration acts differently than configuration -1 for daylight performance. Unlike configuration -1 , in configuration-2, floor area (covered by illumination above threshold) reduction is higher for higher WWRs, i.e. for WWR 73\% to WWR 55\%. Glazing typology and WWR have significant influence on reduction in floor area covered by target illuminance. Reduction percentage is lower when both courtyard and skylight's glazing are double clear and WWR is large. Reduction percentage is higher when both courtyard and skylight's glazing is low e coated and WWR is small. 
Table 25 Daylighting condition plotted for courtyard glazing Double clear, $6 \mathrm{~mm} / 13 \mathrm{~mm}$ air and sky light Double clear, $6 \mathrm{~mm} / 13 \mathrm{~mm}$ air in configuration - 1

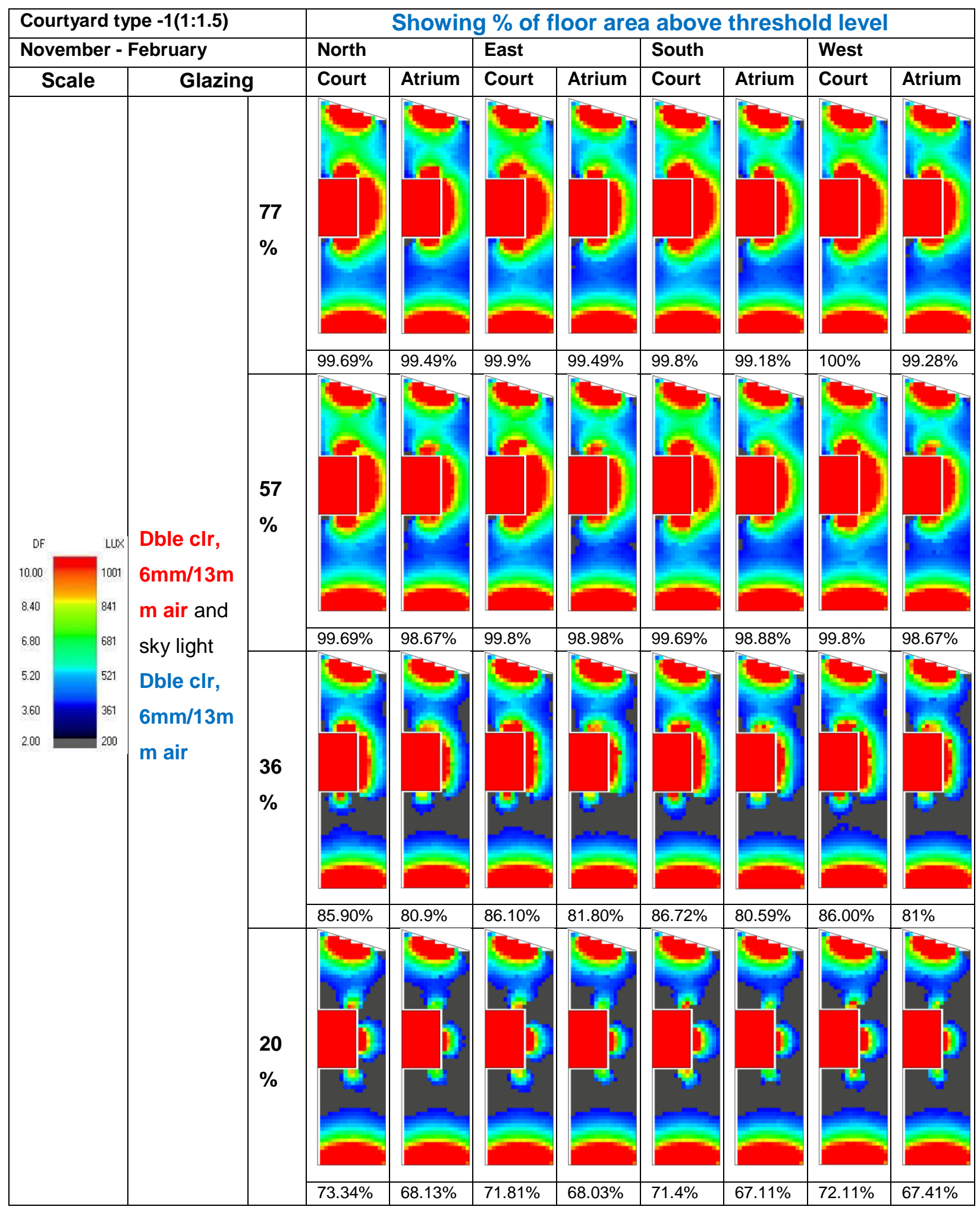




\subsection{Combined calculation of energy consumption and illumination for optimization}

Reduction in energy consumption by atrium option in row houses during winter also resulting in reduction in floor area covered by target illuminance. But the intension of this research is not only reduction of energy consumption, but also find the solution for increased daylighting inside the house. The results show that the options which are performing better for reduction in energy consumption (i.e. consumes less energy during winter), not always a better performer for daylighting inside the row house (less reduction in daylit floor area with target illuminance). So, an optimization is required to find a balance between increased energy consumption and decreased illuminance level to maximize the benefits.

From the simulation done by using Design Builder software, 256 combinations or options are generated for different variables to see the effect on energy consumption and daylighting condition inside the row house, which can be summarized in the following table:

Table 26 Total combination from different variables

\begin{tabular}{|c|c|c|c|c|c|}
\hline Configurations & Orientation & WWR & Courtyard glazing type & Skylight glazing type & \\
\hline \multirow{4}{*}{ Configuration-1 } & North & 77 & Dble clr, $6 \mathrm{~mm} / 13 \mathrm{~mm}$ air & Dble clr, $6 \mathrm{~mm} / 13 \mathrm{~mm}$ air & \multirow{4}{*}{$\begin{array}{l}\text { Combina } \\
\text { tions for } \\
\text { configura } \\
\text { tion } 1 \text { is }\end{array}$} \\
\hline & East & 57 & Dbl LoE (e2=.1) Clr $6 \mathrm{~mm} / 13 \mathrm{~mm}$ Air & Dbl LoE (e2=.1) Clr $6 \mathrm{~mm} / 13 \mathrm{~mm}$ Air & \\
\hline & South & 36 & $\operatorname{Trp~Clr~3mm/13mm~Air~}$ & & \\
\hline & West & 20 & $\operatorname{Trp} \operatorname{LoE}(\mathrm{e} 2=\mathrm{e} 5=.1) \mathrm{Clr} 3 \mathrm{~mm} / 13 \mathrm{~mm}$ Air & & \\
\hline Total option & 4 & 4 & 4 & 2 & 128 \\
\hline \multirow{4}{*}{ Configuration-2 } & North & 73 & Dble clr, $6 \mathrm{~mm} / 13 \mathrm{~mm}$ air & Dble clr, $6 \mathrm{~mm} / 13 \mathrm{~mm}$ air & \multirow{5}{*}{$\begin{array}{l}\text { Combina } \\
\text { tions for } \\
\text { configura } \\
\text { tion } 2 \text { is } \\
128\end{array}$} \\
\hline & East & 55 & Dbl LoE (e2=.1) Clr $6 \mathrm{~mm} / 13 \mathrm{~mm}$ Air & Dbl LoE (e2=.1) Clr $6 \mathrm{~mm} / 13 \mathrm{~mm}$ Air & \\
\hline & South & 36 & Trp Clr 3mm/13mm Air & & \\
\hline & West & 23 & $\operatorname{Trp} \operatorname{LoE}(\mathrm{e} 2=\mathrm{e} 5=.1) \mathrm{Clr} 3 \mathrm{~mm} / 13 \mathrm{~mm}$ Air & & \\
\hline Total option & 4 & 4 & 4 & 2 & \\
\hline
\end{tabular}


In this research, 4 areas are specified (based on outcome of the results) where these combinations show varied results. These areas are as below:

Table 27 Areas of result's outcome

\begin{tabular}{|l|l|}
\hline Area & Outcome \\
\hline Area A & $\begin{array}{l}\text { Percentage of energy reduction by each atrium configuration compared to } \\
\text { similar courtyard configuration }\end{array}$ \\
\hline Area B & Total energy consumption by each atrium option during winter \\
\hline Area C & $\begin{array}{l}\text { Percentage of reduction of area covered by target illuminance for each } \\
\text { configuration }\end{array}$ \\
\hline Area D & \begin{tabular}{l} 
Percentage of area covered by target illuminance for each atrium option \\
\hline
\end{tabular} \\
\hline
\end{tabular}

Since the values of each area is in different units (like, $\mathrm{kWh} / \mathrm{m} 2$, percentages) and varied ranges, all the calculations are required to convert into comparable format by normalization. In one side higher values (like, floor area above target illuminance) represent better outcome, on the other side lower values (like, energy consumption by atriums) represent better outcomes. Hence to resolve this issue, the results are converted into 0 to 10 scale where for both cases higher values represent better outcome. After that, priority weightage is assigned for each area for better optimization. We can see in atrium house heating energy reduction is more than the reduction in floor area covered by target illuminance compared to courtyard house. So, weightage "2" has been assigned for Area A and Area B, weightage "1" for Area C and Area D. Applying all the weightage to the normalized values, the values for 4 areas are added for each configuration. Thus, after sorting in descending order i.e. from higher values of combinations, optimum options are identified. Detailed table for this calculation is attached in appendix. The table below shows the 10 best options determined from optimization. 
Table 28 Table showing ten best optimized combinations

\begin{tabular}{|c|c|c|c|c|c|c|c|c|c|}
\hline $\begin{array}{c}\text { Best } \\
\text { options }\end{array}$ & Configutration & $\begin{array}{l}\text { Window to } \\
\text { Wall Ratio }\end{array}$ & Orientation & Courtyard glazing type & Skylight glazing type & $\begin{array}{c}\text { Energy } \\
\text { consumption } \\
\mathrm{kWh} / \mathrm{m} 2\end{array}$ & $\begin{array}{c}\text { Consumption } \\
\text { reduction \% }\end{array}$ & $\begin{array}{c}\text { Area Covered } \\
\text { by target } \\
\text { illuminance (\%) }\end{array}$ & $\begin{array}{c}\text { Reduction in area } \\
\text { covered by target } \\
\text { illuminance (\%) }\end{array}$ \\
\hline 1 & Config -1 & $77 \%$ & North & Dbl clr $6 \mathrm{~mm} / 13 \mathrm{~mm}$ air & Dbl LoE (e2=.1) Clr $6 \mathrm{~mm} / 13 \mathrm{~mm}$ Air & 97.3 & 21.2 & 99 & 0.9 \\
\hline 2 & Config -2 & $73 \%$ & North & Dbl clr $6 \mathrm{~mm} / 13 \mathrm{~mm}$ air & Dbl LoE (e2=.1) Clr $6 \mathrm{~mm} / 13 \mathrm{~mm}$ Air & 93.6 & 21.4 & 92.1 & 7.3 \\
\hline 3 & Config -1 & $77 \%$ & North & Dbl LoE (e2=.1) Clr $6 \mathrm{~mm} / 13 \mathrm{~mm}$ Air & Dbl LoE (e2=.1) Clr $6 \mathrm{~mm} / 13 \mathrm{~mm}$ Air & 93.1 & 16.2 & 98.2 & 1.6 \\
\hline 4 & Config -1 & $77 \%$ & North & Trp Clr 3mm/13mm Air & Dbl LoE (e2=.1) Clr $6 \mathrm{~mm} / 13 \mathrm{~mm}$ Air & 92.4 & 15.6 & 98.1 & 1.8 \\
\hline 5 & Config -2 & $73 \%$ & North & Dbl LoE (e2=.1) Clr $6 \mathrm{~mm} / 13 \mathrm{~mm}$ Air & Dbl LoE (e2=.1) Clr $6 \mathrm{~mm} / 13 \mathrm{~mm}$ Air & 89.5 & 17.3 & 89.6 & 9.8 \\
\hline 6 & Config - 2 & $73 \%$ & North & Dbl clr $6 \mathrm{~mm} / 13 \mathrm{~mm}$ air & Dble clr, $6 \mathrm{~mm} / 13 \mathrm{~mm}$ air & 94.9 & 20.3 & 92.2 & 7.2 \\
\hline 7 & Config -1 & $77 \%$ & North & $\mathrm{Dbl}$ clr $6 \mathrm{~mm} / 13 \mathrm{~mm}$ air & Dble clr, $6 \mathrm{~mm} / 13 \mathrm{~mm}$ air & 99.3 & 19.6 & 99.1 & 0.6 \\
\hline 8 & Config -1 & $57 \%$ & North & $\mathrm{Dbl} \mathrm{clr} 6 \mathrm{~mm} / 13 \mathrm{~mm}$ air & Dbl LoE (e2=.1) Clr $6 \mathrm{~mm} / 13 \mathrm{~mm}$ Air & 97.5 & 18.4 & 98.1 & 1.8 \\
\hline 9 & Config - 2 & $73 \%$ & East & $\mathrm{Dbl}$ clr $6 \mathrm{~mm} / 13 \mathrm{~mm}$ air & Dbl LoE (e2=.1) Clr $6 \mathrm{~mm} / 13 \mathrm{~mm}$ Air & 92.9 & 18 & 91.5 & 7.7 \\
\hline 10 & Config -1 & $77 \%$ & West & Dbl clr $6 \mathrm{~mm} / 13 \mathrm{~mm}$ air & DbI LoE (e2=.1) Clr $6 \mathrm{~mm} / 13 \mathrm{~mm}$ Air & 97 & 17.1 & 99 & 0.9 \\
\hline
\end{tabular}

The result shows that North oriented configuration-1 (i.e. Courtyard $4.5 \mathrm{~m} \times 3 \mathrm{~m}$ ) with 77\% WWR having courtyard glazing Double Clear $6 \mathrm{~mm} / 13 \mathrm{~mm}$ Air and atrium's skylight glazing Dbl LoE (e2=.1) Clr 6mm/13mm Air can administer maximum benefits from atrium in row houses during winter. In this option atrium's energy consumption is 97.3 $\mathrm{kWh} / \mathrm{m} 2$ and it can reduce $21.2 \%$ energy consumption compare to courtyard option and almost $99 \%$ area can be covered by target illuminance. For configuration-2, courtyard with $73 \%$ WWR having Double Clear $6 \mathrm{~mm} / 13 \mathrm{~mm}$ Air glazing and atrium's skylight having Dbl LoE (e2=.1) Clr 6mm/13mm Air glazing can be another optimum solution.

\subsection{Discussion on research findings}

The objective of this research is to determine the appropriateness of designing atriums in row houses for winter and use it as open courtyard during summer. The research is not only concerned about reduction in energy consumption, but also intends to find solutions to improve daylighting condition inside the row houses of Toronto. Though Toronto experience long and cold winter, but we can not underestimate its hot humid summer. So, generalized findings for cold climates from similar type of studies are not appropriate for this location. Use of an existing Toronto row house for analysis shows representative characteristics of row houses in this location and detail input parameter 
in computer aided simulation provides dependable results for this research. Use of most important variables like courtyard's configuration, orientation, window to wall ratio and glazing percentage (as discussed in Chapter -2, Section - 2.6) also ensures that, outcome of this research will be dominant portion with any additional variables. Detail compilation and discussion of existing knowledge base and simulation output are not only contributing to draw the clear picture of research questions, but also add fuels to critical thinking. Each individual result, such as effect of different orientations to energy consumptions, effect of different window wall ratios etc. actually develops a portfolio of variables and their impact on courtyards and atriums of row houses in Toronto. The optimization brings all the contrasts together to make decisions about different combinations to get maximum benefits from design decisions.

\subsubsection{Comparative analysis}

Comparative analysis of individual findings and optimizations explore the new dimension to understand the impact of atriums in replace to courtyard during winter. For better understanding we can restate some individual findings here, such as, in North oriented courtyard configuration-2 with $73 \%$ WWR, courtyard glazing Double clear, $6 \mathrm{~mm} / 13 \mathrm{~mm}$ air, skylight glazing Dbl LoE $(\mathrm{e} 2=.1) \mathrm{Clr} 6 \mathrm{~mm} / 13 \mathrm{~mm}$ Air shows maximum percentage (21.4\%) of energy reduction by atrium option compared to its courtyard option during winter. Another individual result shows that, East oriented atrium configuration -2 with 55\% WWR, courtyard glazing Trp LoE (e2=e5=.1) Clr 3mm/13mm Air and sky light Dbl LoE (e2=.1) Clr 6mm/13mm Air shows lowest energy consumption $(85.9 \mathrm{kWh} / \mathrm{m} 2)$ among all atrium options, but its percentage (10.8\%) of energy reduction in atrium option compared to similar courtyard option is not maximum. For daylighting, 
East oriented configuration-1 with 77\% WWR, courtyard glazing Double clear, $6 \mathrm{~mm} / 13 \mathrm{~mm}$ air, skylight glazing double clear, $6 \mathrm{~mm} / 13 \mathrm{~mm}$ air, percentage of decrease $(0.3 \%)$ in daylit area covered by target illuminance is lowest compared to similar courtyard option. For this combination percentage of floor area covered by target illuminance is maximum (99.489\%). But this individual result creates confusion for designers while he needs to select only one combination. Optimization act as bridge among the dissimilarities and it can provide a comparative information like the following example table. This can help in decision making along with the deep understanding on combined effect of variables.

Table 29 Table showing example comparison between best individual option and best optimum combination

\begin{tabular}{|l|l|l|l|}
\hline \multirow{2}{*}{ Variables } & Best individual option & Best optimum option \\
\cline { 2 - 4 } & For energy & For daylighting & For energy and daylighting together \\
\hline Courtyard configuration & 2 & 1 & 1 \\
\hline Orientaion & North & East & North \\
\hline Window to Wall Ratio & $73 \%$ & $77 \%$ & $77 \%$ \\
\hline Courtyard glazing & Double clear, $6 \mathrm{~mm} / 13 \mathrm{~mm}$ air & Double clear, $6 \mathrm{~mm} / 13 \mathrm{~mm}$ air & $\mathrm{Dbl} \mathrm{clr} 6 \mathrm{~mm} / 13 \mathrm{~mm}$ air \\
\hline Skylight glazing & Dbl LoE $(\mathrm{e} 2=.1) \mathrm{Clr} 6 \mathrm{~mm} / 13 \mathrm{~mm}$ Air & Double clear, 6mm/13mm air & $\mathrm{Dbl} \mathrm{LoE} \mathrm{(e2=.1)} \mathrm{Clr} 6 \mathrm{~mm} / 13 \mathrm{~mm}$ Air \\
\hline
\end{tabular}

The energy performance of atriums in row houses compared to courtyard during winter can be interpreted from the research results. It is almost obvious that atrium options save energy in row houses in winter compared to courtyard. But this research also guides us how maximum benefits can be ensured with different variables. Research result shows, unlike energy performance, daylight condition deteriorates inside the house to some extent in atrium house. For maximizing the reduction in heating energy consumption and minimizing the reduction in floor area covered by target illuminance the best optimum combination is north orientated configuration -1 with WWR $77 \%$, 
courtyard glazing Dbl clr 6mm/13mm air and skylight glazing Dbl LoE (e2=.1) Clr $6 \mathrm{~mm} / 13 \mathrm{~mm}$ Air.

\subsubsection{Connection between research result and existing knowledge}

In cold climates atriums are intentionally used for reducing heat loss, capture solar energy. At the same time atriums bring daylight inside the core of the house. Previous researches also show that the best timing for using atrium option is winter (Chapter -2 , Section- 2.7 and 2.8). This research result finds similar trend for Toronto row houses during winter months. The result also agrees that, atrium is better option for bringing daylight inside the core of the house and the percentage of reduction in daylight is not very significant in atrium options. However, previous study shows that open courtyard thermally performs better in shorter building, but with the increase of building height at certain point atrium performs better (Chapter -2, Section -2.8). This research result shows that $2 \%$ to $21 \%$ reduction in energy consumption is possible by atrium options with its single-story height which shows a clear inclination towards previous research result found in existing knowledge base. There are other issues attached to this ranges. The courtyards and atriums are only located on the upper floor of the two-story row house of this research and it covers only $7.6 \%$ of total floor area of row house, so it can not create huge impact on total area's energy consumption. If we only consider how much energy atrium can reduce during winter which is increased by inclusion of courtyard in row houses, then we find atrium recovers $60 \%$ to $85 \%$ of energy that is increased for courtyard. 


\subsection{Summary of results}

- During winter (peak heating season), row houses with atrium consumes less energy than row houses with courtyards and this reduction ranges from $2.2 \%$ to $21.4 \%$.

- The options that consume more energy in courtyard forms, their percentages of reduction in energy consumptions are also higher in atrium options.

- Reduction in energy consumption by atrium house compared to courtyard house is highest (21.4\%) when double low e coated glazing is used for skylight and double clear glazing is used for courtyard windows. This reduction percentage is lowest $(2.2 \%)$ when triple low e glazing is used for courtyard windows and double clear glazing is used for atrium's skylight.

- Energy reduction percentages are also bigger for larger WWRs. That means, the air is modified in the atrium, house consumes less heating energy when it has bigger window.

- While considering orientation, south oriented atriums consume more energy than others for both configurations. Solar heat gain inside the atrium can reduce heating energy consumption of house during winter. Sun path line lowers towards south in winter. So, south oriented atrium is supposed to gain solar heat from lateral direction. Since this study house is one of the middle units, it can not be benefitted from south orientated atrium.

- Percentage of floor area covered by target illuminance is reduced in row houses with atriums than row houses with courtyards during winter and this reduction ranges from $0.3 \%$ to $23 \%$. 
- The reduction in daylit floor area above target illuminance is maximum when both courtyard and skylight glazing are low e coated with small WWR and reduction percentage is lower when both courtyard and skylight's glazing are double clear with large WWR.

- Best optimum combination for maximum benefit (combinedly from energy and daylighting) is North orientated configuration -1 with WWR $77 \%$, courtyard glazing double clear $6 \mathrm{~mm} / 13 \mathrm{~mm}$ air and skylight glazing double LoE (e2=.1) clear $6 \mathrm{~mm} / 13 \mathrm{~mm}$ Air.

\section{Conclusion and recommendation for future work}

\subsection{Conclusion}

Row houses are an optimum solution for achieving energy efficiency and density in urban area of Toronto. However, this housing typology is suffering from poor daylighting condition in the interior of the houses. Addition of courtyards in row houses of Toronto can improve daylighting condition in the interior, but at the same time this option increases heating energy consumption which is conflicting with the philosophy of row houses. This study investigates the impact of atrium on energy demand and daylighting condition inside the row house during winter. The responses of this research for the research questions are following:

1. How the energy performance and daylighting conditions are impacted in a row house with courtyard in Toronto if the courtyard is converted into atrium during winter? 
The heating energy demand that is increased inside the row house due to incorporation of courtyard can be reduced by converting this courtyard into atrium during winter. The reduction in energy consumption of atrium house compared to courtyard house is ranges from $2.2 \%$ to $21.4 \%$ depending on different glazing typology, WWRs and orientations. However, this atrium also effects the daylighting condition by reducing daylit floor area above target illuminance inside the house. This reduction in floor area covered by target illuminance is not very significant and ranges from $0.3 \%$ to $17 \%$ based on different WWRs and glazing typology.

2. How to maximize the benefits through design decisions in row house with atrium by minimizing energy consumption and increasing daylighting during winter in Toronto?

Optimum combination for designing atrium in row houses for winter may maximize the benefits by reducing energy consumption and increasing daylight inside the house. Bigger courtyard having large window to wall ratio, clear glazing for courtyard windows and low e coated glazing for atrium's skylight can create a balance between increased energy consumption and decreased daylighting inside the house. Specifically, best optimum combination found in this research is north orientated bigger atrium configuration with WWR $77 \%$, courtyard glazing double clear $6 \mathrm{~mm} / 13 \mathrm{~mm}$ air and skylight glazing double LoE $(\mathrm{e} 2=.1)$ clear $6 \mathrm{~mm} / 13 \mathrm{~mm}$ Air.

However, there is also an additional cost associated with atrium construction. Therefore, a cost benefit analysis is also necessary to determine its feasibility, but this analysis is not covered by this study. Besides, study on other shapes of row houses may differ in 
results, so it needs to be studied to generalize the findings for this housing typology in Toronto.

\subsection{Recommended future work}

In this research only one row house is studied. But the result may very for other shapes of the row houses of Toronto with different courtyard configurations. So, a further study can be done to see the impact of different shapes of row houses on energy consumption.

This study only used static metric to evaluate the day lighting condition in row houses with atrium. However, the study of the daylighting condition in atrium house with dynamic metric can develop a better understanding about daylight performance of such courtyards and atriums in row houses.

Shape of atrium's skylight also influence the energy and daylight performance. So, the similar study can be testified with different shapes of skylight to determine the difference in energy consumption. 


\section{References}

Advanced Buildings. (2018). Daylighting pattern guide. Retrieved from https://patternguide.advancedbuildings.net/home

Aldawoud, A. (2008). Thermal performance of courtyard buildings. Energy and Buildings, 40, 906-910. doi:10.1016/j.enbuild.2007.07.007

Aldawoud, A. (2013). The influence of the atrium geometry on the building energy performance. Energy and Buildings, 57, 1-5.

Aldawoud, A., \& Clark, R. (2008). Comparative analysis of energy performance between courtyard and atrium in buildings. Energy and Buildings, 40, 209-214.

Al-Hemiddi, N. A., \& Megren Al-Saud, K. A. (2001). The effect of a ventilated interior courtyard on the thermal performance of a house in a hot-arid region. Renewable Energy, 24(3), 581-595.

Atif, M. R. (1994). Top-glazed public spaces: Amenities, energy costs and indoor environment. Construction Canada, 36, 43-47.

Ayoob, A. N., \& Izard, J. L. (1994). Study of comfort in atrium design. Renewable Energy, 5(5-8), 1002-1005.

Blaszak, K. M. (2010). Towards sustainability : Prioritizing retrofit options for toronto's single-family homes. (Unpublished MASc). Ryerson University, Toronto, Canada. 
CMHC. (2014). CHS-demography 2013. ().Canadian Mortgage and Housing Corporation. Retrieved from http://www.cmhcschl

Crawley, D., \& et al. (2004). EnergyPlus: New, capable, and linked. Journal of Architecture and Planning Research, 21(4), 292-302.

Culjat, B. (1988). Climate responsive social space: A scandinavian perspective. In J. Mänty, \& N. Pressman (Eds.), Cities designed for winter climate (1st ed., pp. 346363). Helsinki: Building Book.

Cuttle, C. (2003). Ligthing by design. Oxford: Architectural Press.

De la Riva, R., Gagnon, S., \& Affleck, G. (2000). "Plex" housing : A renewed tradition. (). Ottawa: CMHC.

DesignBuilder software guide. (2009). DesignBuilder simulation +CFD training guide. ().DesignBuilder.

Edwards, B., Sibley, M., Hakmi, M., \& Land, P. (Eds.). (2006). Courtyard housing: Past, present and future (First ed.). New York: Taylor \& Francis.

Fathy, H. (1986). Natural energy and vernacular architecture: Principles and examples with reference to hot arid climates. Chicago: The University of Chicago Press.

Friedman, A. (2012). Town and terraced housing: For affordability and sustainability. New York: Routledge. 
Guzowski, M. (2010). Towards zero energy architecture - new solar design. London: Laurence King.

Haw, L. C., Saadatian, O., Sulaiman, M. Y., Mat, S., \& Sopian, K. (2012). Empirical study of a wind-induced natural ventilation tower under hot and humid climatic conditions. Energy and Buildings, 52, 28-38.

Issi, F., \& Kaplan, O. (2018). The determination of load profiles and power consumptions of home appliances. Energies, 11(607) doi:10.3390/en11030607

Jermyn, D. (2014). Deep energy retrofits: Toronto's urban single family housing stock. (Unpublished MASc). Ryerson University, Toronto, Canada.

Laouadi, A., Atif, M. R., \& Galasiu, A. (2003). Methodology towards developing skylight design tools for thermal and energy performance of atriums in cold climates. Building and Environment, 38(1), 117-127.

Laouadi, A., Atif, M., \& Galasiu, A. (2002). Towards developing skylight design tools for thermal and energy performance of atriums in cold climates. Building and Environment, 37, 1289-1316.

Liu, D. (1988). A review of Harbin's challenge to winter. In J. Mänty, \& N. Pressman (Eds.), Cities designed for winter climates (1st ed., pp. 113-129). Helsinki: Building Book.

Mänty, J., \& Pressman, N. (1988). Cities designed for winter Building Book Ltd. 
Martin, L., \& March, L. (Eds.). (1972). Urban space and structures. UK: Cambridge University Press.

Meir, I. A., Pearlmutter, D., \& Etzion, Y. (1995). On the microclimatic behavior of two semi enclosed attached courtyards in a hot dry region. Building and Environment, 30(4), 563-572.

Mills, F. (1991). Atrium buildings: Architecture and engineering. Construction Industry Conference Centre Ltd, Welwyn, UK, , 13-22.

Muhaisen, A. S. (2006). Shading simulation of the courtyard form in different climatic regions. Building and Environment, 41(12), 1731-1741. doi:10.1016/j.buildenv.2005.07.016

Muhaisen, A. S., \& Gadi, M. B. (2006). Effect of courtyard proportions on solar heat gain and energy requirement in the temperate climate of rome. Building and Environment, 41(3), 245-253. doi:10.1016/j.buildenv.2005.01.031

OBC. (2006). Ontario ministry of municipal affairs and housing. ().Ontario Building Code.

OBC. (2012). Ontario ministry of municipal affairs and housing. (). Ontario Building Code Supplementary Standard 12, 20.

Oliver, P. (2003). Dwellings: The house across the world. Oxford: Phaidon Press Ltd. 
Özkan, S. (2005). Courtyard: A typology that symbolises a culture. In B. Edwards, M. Sibley, M. Hakmi \& P. Land (Eds.), Housing. past, present and future (pp. 373). New York: Taylor \& Francis.

Petruccioli, A. (2006). The courtyard house: Typological variations. In B. Edwards, M. Sibley, M. Hakmi \& P. Land (Eds.), Courtyard housing. past, present and future (). New York: Taylor \& Francis.

Pfeifer, G., \& Brauneck, P. (2008). Courtyard houses: A housing typology. Boston: Birkhäuse.

Radhi, H. (2010). On the optimal selection of wall cladding system to reduce direct and indirect $\mathrm{CO} 2$ emissions.

. Energy, 35, 1412-1424.

Rajapaksha, I., Nagai, H., \& Okumiya, M. (2003). A ventilated courtyard as a passive cooling strategy in the warm humid tropics. Renewable Energy, 28(11), 1755-1778.

Ratti, C., Raydan, D., \& Steemers, K. (2003). Building form and environmental performance: Archetypes, analysis and an arid climate. Energy and Buildings, 35, 49-59.

Raydan, D., Ratti, C., \& Steemers, K. (2003). Building form and environmental performance: Archetypes, analysis and an arid climate. Energy and Buildings, 35(1), 49-59. doi:10.1016/S0378-7788(02)00079-8 
Snøhetta (Architectural firm). (2018). Design of the ZEB pilot house, larvik, norway. Retrieved from http://snohetta.com/project/188-zeb-pilot-house

Steemers, K., Baker, N., Crowther, D., Dubiel, J., Nikolopoulou, M., \& Ratti, C. (1997). City texture and microclimate. Urban Design Study, 3, 25-50.

Tabesh, T., \& Sertyesilisik, B. (2016). An investigation into energy performance with the integrated usage of a courtyard and atrium. Buildings, 6(21) doi:10.3390/buildings6020021

Taleghani, M., Tenpierik, M., \& Dobbelsteen, A. (2014). Energy performance and thermal comfort of courtyard/atrium dwellings in the netherlands in the light of climate change. Renewable Energy, 63, 486-497.

U.S. Energy Information Administration. (2018). International energy data and analysis. Retrieved from https: //www.Eia.Gov/beta/international/analysis

Vaisman, G. (2015). Influence of internal courtyards on the energy load and hours of illuminance in row houses in toronto (MBSc).

Ward, W. P. (1999). A history of domestic space: Privacy and the canadian home. Vancouver: UBC Press.

Zirnhelt, H. E. (2013). Using calibrated simulation to quantify the energy savings from residential passive solar design in canada. (Unpublished MASc). Ryerson University, Toronto, Canada. 


\section{Appendices}

Appendix I Energy consumption by configuration -1, Option - 1

\begin{tabular}{|c|c|c|c|c|c|c|c|c|c|c|c|c|c|c|c|c|c|c|c|}
\hline \multicolumn{4}{|c|}{ Courtyard type -1 (1:1.5) } & \multicolumn{4}{|c|}{ North } & \multicolumn{4}{|c|}{ East } & \multicolumn{4}{|c|}{ South } & \multicolumn{4}{|c|}{ West } \\
\hline Novembe & r-April energy & & Option & Elec(kWh) & Gas(kWh) & $k W h / m 2$ & Decrease\% & Elec(kWh) & ) Gas(kWh) & $k W h / m 2$ & Decrease\% & $\%$ Elec(kWh) & Gas(kWh) & $\mathrm{kWh} / \mathrm{m} 2$ & Decrease\% & Elec(kWh) & 1) Gas(kWh) & $\mathrm{kWh} / \mathrm{m} 2$ & Decrease $\%$ \\
\hline \multirow{8}{*}{ Glazing } & \multirow{8}{*}{$\begin{array}{l}\text { Courtyard Dble clr, } \\
6 \mathrm{~mm} / 13 \mathrm{~mm} \text { air } \\
\text { and sky light Dble } \\
\mathrm{clr}, 6 \mathrm{~mm} / 13 \mathrm{~mm} \\
\text { air }\end{array}$} & \multirow{2}{*}{$77 \%$} & Court & 2297.31 & 17755.55 & 123.45 & -20 & 2236.55 & 16499.58 & 115.35 & -15 & 2328.33 & 16242.13 & 114.33 & -11 & 2255.05 & 16768.04 & 117.11 & -15 \\
\hline & & & Atrium & 2325.25 & 13802.45 & 99.29 & & 2327.27 & 13685.44 & 98.58 & & 2326.73 & 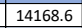 & 101.55 & & 2327.91 & 13755.19 & 99.01 & \\
\hline & & \multirow{2}{*}{$57 \%$} & Court & 2273.78 & 17136.29 & 119.49 & -17 & 2207.76 & 16097.84 & 112.69 & -12 & 2292.3 & 16062.7 & 113 & -10 & 2215.18 & 16359.63 & 114.35 & -13 \\
\hline & & & Atrium & & 13825.06 & 99.48 & & 2336.55 & 13710.53 & 98.79 & & 2336.17 & 14188.14 & 101.73 & & 37.37 & 13779.45 & 99.22 & \\
\hline & & \multirow{2}{*}{$36 \%$} & Court & 2227.91 & 18019.28 & 124.65 & -15 & \begin{tabular}{|l|}
2161.37 \\
\end{tabular} & 17937.77 & 123.74 & -15 & 2228.35 & \begin{tabular}{|l|}
17198.3 \\
\end{tabular} & 119.6 & -10 & 2182.55 & 16880.7 & 117.36 & -10 \\
\hline & & & Atrium & 2360.85 & 14844.64 & 105.92 & & 2363.77 & 14758.44 & 105.41 & & 2363.04 & 15214.04 & 108.21 & & 2364.73 & 14828.4 & 105.85 & \\
\hline & & \multirow{2}{*}{$20 \%$} & Court & 2228.11 & 16043.38 & 112.48 & -11 & \begin{tabular}{|l|}
2146.85 \\
\end{tabular} & 15582.01 & 109.14 & -9 & 2226.23 & 16091.68 & 112.77 & -9 & \begin{tabular}{|l}
2131.77 \\
\end{tabular} & 15843.25 & 110.66 & -10 \\
\hline & & & Atrium & 2350.37 & \begin{tabular}{|l|}
13869.59 \\
\end{tabular} & 99.86 & & 2353.31 & \begin{tabular}{|l}
13761.46 \\
\end{tabular} & 99.21 & & 2352.73 & 14225.05 & 102.06 & & 2354.31 & 13829.16 & 99.63 & \\
\hline
\end{tabular}

Appendix II Energy consumption by configuration -1, Option - 2

\begin{tabular}{|c|c|c|c|c|c|c|c|c|c|c|c|c|c|c|c|c|c|c|c|}
\hline \multicolumn{4}{|c|}{ Courtyard type -1 (1:1.5) } & \multicolumn{4}{|c|}{ North } & \multicolumn{4}{|c|}{ East } & \multicolumn{4}{|c|}{ South } & \multicolumn{4}{|c|}{ West } \\
\hline \multicolumn{3}{|c|}{ November - April energy } & Option & Elec & Gas & $k W h / m 2$ & Decrease\% & Elec & Gas & $k W h / m 2$ & Decrease\% & Elec & Gas & $\mathrm{kWh} / \mathrm{m} 2$ & Decrease\% & Elec & Gas & $\mathrm{kWh} / \mathrm{m} 2$ & Decrease\% \\
\hline \multirow{8}{*}{ Glazing } & \multirow{8}{*}{$\begin{array}{l}\text { Courtyard Dble clr, } \\
6 \mathrm{~mm} / 13 \mathrm{~mm} \text { air } \\
\text { and sky light Dbl } \\
\text { LoE (e2=.1) Clr } \\
6 \mathrm{~mm} / 13 \mathrm{~mm} \text { Air }\end{array}$} & \multirow{2}{*}{$77 \%$} & Court & 2297.31 & 17755.55 & 123.45 & \begin{tabular}{|l|}
-21 \\
\end{tabular} & 2236.55 & 16499.58 & 115.35 & \begin{tabular}{|l|}
-16 \\
\end{tabular} & 2328.33 & 16242.13 & 114.33 & -13 & 2255.05 & 16768.04 & 117.11 & -17 \\
\hline & & & Atrium & 2281.79 & 13525.11 & 97.31 & & 2283.64 & 13407.71 & 96.6 & & 2283.34 & 13893.22 & 99.59 & & 2284.31 & 13478.56 & 97.04 & \\
\hline & & \multirow{2}{*}{$57 \%$} & Court & 2273.78 & 17136.29 & 119.49 & -18 & 2207.76 & 16097.84 & 112.69 & -14 & 2292.3 & 16062.7 & 113 & -12 & 2215.18 & 16359.63 & 114.35 & -15 \\
\hline & & & Atrium & 2289.72 & 13552.69 & 97.53 & & 2291.72 & 13437.4 & 96.83 & & 2291.52 & 13916.96 & 99.78 & & 2292.54 & 13507.71 & 97.27 & \\
\hline & & \multirow{2}{*}{$36 \%$} & Court & 2227.91 & 18019.28 & 124.65 & -17 & 2161.37 & 17937.77 & 123.74 & -16 & 2228.35 & 17198.3 & 119.6 & -11 & 2182.55 & 16880.7 & 117.36 & -11 \\
\hline & & & Atrium & 2314.67 & 14575.55 & 103.98 & & 2317.56 & 14489.44 & 103.47 & & 2316.93 & 14946.12 & 106.28 & & 2318.61 & 14560.16 & 103.91 & \\
\hline & & \multirow{2}{*}{$20 \%$} & Court & 2228.11 & 16043.38 & 112.48 & -13 & 2146.85 & 15582.01 & & -11 & 2226.23 & 16091.68 & 112.77 & -11 & 2131.77 & 15843.25 & 110.66 & -12 \\
\hline & & & Atrium & 2303.2 & 13602.66 & 97.92 & & 2306.17 & 13494.28 & 97.27 & & 2305.67 & 13958.26 & 100.13 & & 2307.25 & 13562.03 & 97.7 & \\
\hline
\end{tabular}

Appendix III Energy consumption by configuration -1, Option - 3

\begin{tabular}{|c|c|c|c|c|c|c|c|c|c|c|c|c|c|c|c|c|c|c|c|}
\hline \multicolumn{4}{|c|}{ Courtyard type -1 (1:1.5) } & \multicolumn{4}{|c|}{ North } & \multicolumn{4}{|c|}{ East } & \multicolumn{4}{|c|}{ South } & \multicolumn{4}{|c|}{ West } \\
\hline Novembe & r- April energy & & Option & Elec & Gas & $k W h / m 2$ & Decrease\% & Elec & Gas & kWh/m2 & Decrease\% & Elec & Gas & $k W h / m 2$ & Decrease\% & Elec & Gas & $\mathrm{kWh} / \mathrm{m} 2$ & Decrease\% \\
\hline \multirow{8}{*}{ Glazing } & \multirow{8}{*}{\begin{tabular}{|c|} 
Courtyard Dbl LoE \\
$(\mathrm{e} 2=.1) \mathrm{Clr}$ \\
$6 \mathrm{~mm} / 13 \mathrm{~mm}$ Air \\
and sky light Dbl \\
Clr $6 \mathrm{~mm} / 13 \mathrm{~mm}$ Air
\end{tabular}} & \multirow{2}{*}{$77 \%$} & Court & 2227.07 & 15816 & 111.08 & \begin{tabular}{|l|}
-14 \\
\end{tabular} & 2179.97 & 14796.05 & 104.51 & -10 & 2250.7 & 14578.82 & 103.61 & \begin{tabular}{|c|}
-6 \\
\end{tabular} & 2193.11 & 15009.71 & 105.91 & -10 \\
\hline & & & Atrium & 2316.02 & 13127.74 & 95.08 & & 2318.96 & 13035.42 & 94.53 & & 2317.75 & 13422.77 & 96.9 & & 2319.93 & 13090.65 & 94.87 & \\
\hline & & \multirow{2}{*}{$57 \%$} & Court & 2211.13 & 15477.15 & 108.89 & -13 & 2156.08 & 14643.35 & 103.42 & -8 & 2220.74 & 14603.79 & 103.58 & -6 & 2160.82 & 14850.61 & 104.73 & -9 \\
\hline & & & Atrium & 2325.54 & 13149.84 & 95.27 & & 2328.57 & 13059.1 & 94.73 & & 2327.46 & 13440.85 & 97.07 & & 2329.63 & 13113.51 & 95.07 & \\
\hline & & \multirow{2}{*}{$36 \%$} & Court & 2180.21 & 16908.65 & 117.52 & -14 & 2132.37 & 16847.49 & 116.85 & -13 & 2182.29 & 16240.52 & 113.42 & -9 & 2146.2 & 15991.35 & 111.66 & -9 \\
\hline & & & Atrium & 2352.46 & $\mid 14153.4$ & 101.62 & & 2356.22 & 14090.08 & 101.25 & & 2354.73 & \begin{tabular}{|l|l}
14450.49 \\
\end{tabular} & 103.46 & & 2357.35 & \begin{tabular}{|l|l}
14145.83 \\
\end{tabular} & 101.6 & \\
\hline & & \multirow{2}{*}{$20 \%$} & Court & 2175.08 & 14975.38 & 105.58 & -9 & 2110.82 & 14600.99 & 102.88 & -8 & 2170.89 & \begin{tabular}{|l|l}
15015.28 \\
\end{tabular} & 105.8 & -8 & 2098.86 & 14805.81 & 104.07 & -8 \\
\hline & & & Atrium & \begin{tabular}{|l|}
2342.51 \\
\end{tabular} & \begin{tabular}{|l|l} 
\\
\end{tabular} & 95.61 & & \begin{tabular}{|l|}
2346.21 \\
\end{tabular} & \begin{tabular}{|l}
13103.31 \\
\end{tabular} & 95.11 & & 2344.87 & \begin{tabular}{|l|}
13473.06 \\
\end{tabular} & 97.38 & & \begin{tabular}{|l|}
2347.37 \\
\end{tabular} & $\mid 13158.02$ & 95.46 & \\
\hline
\end{tabular}

Appendix IV Energy consumption by configuration -1, Option - 4

\begin{tabular}{|c|c|c|c|c|c|c|c|c|c|c|c|c|c|c|c|c|c|c|c|}
\hline \multirow{2}{*}{\multicolumn{3}{|c|}{\begin{tabular}{|c|} 
Courtyard type -1 (1:1.5) \\
November - April energy
\end{tabular}}} & & \multicolumn{4}{|c|}{ North } & \multicolumn{4}{|c|}{ East } & \multicolumn{4}{|c|}{\begin{tabular}{|l|} 
South \\
\end{tabular}} & \multicolumn{4}{|c|}{ West } \\
\hline & & & & Elec & Gas & $k W h / m 2$ & Decrease\% & Elec & Gas & kWh/m2 & Decrease\% & Elec & Gas & $k W h / m 2$ & Decrease\% & Elec & Gas & $\mathrm{kWh} / \mathrm{m} 2$ & Decrease $\%$ \\
\hline \multirow{8}{*}{ Glazing } & \multirow{8}{*}{$\begin{array}{c}\text { Courtyard Dbl LoE } \\
\text { (e2=.1) Clr } \\
6 \mathrm{~mm} / 13 \mathrm{~mm} \text { Air } \\
\text { and sky light Dbl } \\
\text { LoE (e2=.1) Clr } \\
6 \mathrm{~mm} / 13 \mathrm{~mm} \text { Air }\end{array}$} & \multirow{2}{*}{$77 \%$} & Court & 2227.07 & 15816 & 111.08 & \begin{tabular}{|l|}
-16 \\
\end{tabular} & 2179.97 & 14796.05 & 104.51 & \begin{tabular}{l|l}
-11 \\
\end{tabular} & 2250.7 & 14578.82 & 103.61 & -8 & 2193.11 & 15009.71 & 105.91 & -12 \\
\hline & & & Atrium & 2272.18 & 12853.64 & 93.12 & & 2274.97 & 12761.29 & 92.57 & & 2273.98 & 13148.85 & 94.95 & & 2275.95 & 12816.83 & 92.92 & \\
\hline & & & Court & 2211.13 & 15477.15 & 108.89 & -14 & 2156.08 & 14643.35 & 103.42 & -10 & 2220.74 & 14603.79 & 103.58 & -8 & 2160.82 & 14850.61 & 104.73 & -11 \\
\hline & & & Atrium & 2280.42 & 12878.68 & 93.32 & & 2283.34 & 12787.36 & 92.78 & & 2282.43 & 13170.74 & 95.13 & & 2284.41 & 12843.13 & 93.13 & \\
\hline & & & Court & 2180.21 & 16908.65 & 117.52 & -15 & 2132.37 & 16847.49 & & -15 & 2182.29 & 16240.52 & & -10 & 2146.2 & 15991.35 & 111.66 & -11 \\
\hline & & & Atrium & 2306.05 & 13885.76 & 99.68 & & 2309.8 & 13822.5 & 99.32 & & 2308.4 & 14183.64 & 101.53 & & 2311.01 & \begin{tabular}{|l|}
13878.26 \\
\end{tabular} & 99.67 & \\
\hline & & \multirow{2}{*}{$20 \%$} & Court & 2175.08 & \begin{tabular}{|l|l}
14975.38 \\
\end{tabular} & 105.58 & -11 & 2110.82 & 14600.99 & 102.88 & -9 & 2170.89 & \begin{tabular}{|l|l}
15015.28 \\
\end{tabular} & 105.8 & -10 & 2098.86 & 14805.81 & 104.07 & -10 \\
\hline & & & Atrium & 2295.05 & \begin{tabular}{|l}
12922.25 \\
\end{tabular} & 93.68 & & \begin{tabular}{|l|}
2298.76 \\
\end{tabular} & \begin{tabular}{|l|}
12836.53 \\
\end{tabular} & 93.18 & & 2297.58 & \begin{tabular}{|l|}
13206.73 \\
\end{tabular} & 95.45 & & \begin{tabular}{|l}
2300.02 \\
\end{tabular} & \begin{tabular}{|l|}
12891.89 \\
\end{tabular} & 93.53 & \\
\hline
\end{tabular}

Appendix V Energy consumption by configuration -1, Option - 5

\begin{tabular}{|c|c|c|c|c|c|c|c|c|c|c|c|c|c|c|c|c|c|c|c|}
\hline \multirow{2}{*}{\multicolumn{3}{|c|}{\begin{tabular}{|c|}
\multicolumn{2}{|c|}{ Courtyard type -1 (1:1.5) } \\
November - April energy \\
\end{tabular}}} & & \multicolumn{4}{|c|}{ North } & \multicolumn{4}{|c|}{ East } & \multicolumn{4}{|c|}{ South } & \multicolumn{4}{|c|}{ West } \\
\hline & & & & Elec & Gas & $k W h / m 2$ & Decrease\% & Elec & Gas & $k W h / m 2$ & Decrease\% & Elec & Gas & kWh/m2 & Decrease\% & Elec & Gas & kWh/m2 & Decrease\% \\
\hline \multirow{8}{*}{ Glazing } & \multirow{8}{*}{$\begin{array}{c}\text { Courtyard Trp Clr } \\
3 \mathrm{~mm} / 13 \mathrm{~mm} \text { Air } \\
\text { and sky light Dbl } \\
\text { Clr } 6 \mathrm{~mm} / 13 \mathrm{~mm} \text { Air }\end{array}$} & \multirow{2}{*}{$77 \%$} & Court & 2280.07 & 15505.94 & 109.5 & \begin{tabular}{|l|}
-14 \\
\end{tabular} & 2213.58 & 14310.22 & 101.73 & -8 & 2301.93 & 14083.08 & \begin{tabular}{|l|}
100.87 \\
\end{tabular} & -4 & 2231.83 & 14547.12 & 103.3 & -9 \\
\hline & & & Atrium & 2320.53 & 13005.32 & 94.35 & & 2322.81 & 12884.73 & 93.62 & & 2322.24 & 13347.74 & 96.47 & & 2323.78 & 12949.97 & 94.03 & \\
\hline & & \multirow{2}{*}{$57 \%$} & Court & 2262.17 & 15147.28 & 107.18 & -12 & 2187.08 & 14156.96 & 100.62 & -7 & 2268.75 & 14140.71 & 101.02 & -4 & 2195.15 & 14389.06 & 102.1 & -8 \\
\hline & & & Atrium & \begin{tabular}{|l|}
2329.7 \\
\end{tabular} & 13029.5 & 94.56 & & 2332.11 & 12911.22 & 93.84 & & 2331.61 & 13367.85 & 96.65 & & 2333.19 & \begin{tabular}{|l|}
12975.9 \\
\end{tabular} & 94.25 & \\
\hline & & \multirow{2}{*}{$36 \%$} & Court & 2215.88 & 16654.76 & 116.17 & -13 & 2153.3 & 16570.27 & 115.27 & -13 & 2220.13 & 15876.87 & 111.41 & -8 & 2169.28 & 15561.35 & 109.16 & -8 \\
\hline & & & Atrium & 2355.93 & 14035.63 & 100.91 & & 2359.29 & 13946.52 & 100.38 & & 2358.28 & 14380.07 & 103.05 & & 2360.45 & 14011.47 & 100.79 & \\
\hline & & \multirow{2}{*}{$20 \%$} & Court & 2219.04 & 14633.09 & 103.75 & -8 & 2132.54 & 14180.89 & 100.43 & -6 & 2210.66 & 14686.42 & 104.02 & -7 & 2118.77 & \begin{tabular}{|l|}
14419.4 \\
\end{tabular} & 101.81 & -7 \\
\hline & & & Atrium & 2345.67 & \begin{tabular}{|l|}
13076.28 \\
\end{tabular} & 94.94 & & 2348.98 & 12964.65 & 94.28 & & 2348.1 & 13404.83 & 96.98 & & 2350.2 & 13028 & 94.67 & \\
\hline
\end{tabular}


Appendix VI Energy consumption by configuration -1, Option - 6

\begin{tabular}{|c|c|c|c|c|c|c|c|c|c|c|c|c|c|c|c|c|c|c|c|}
\hline \multirow{2}{*}{\multicolumn{3}{|c|}{\begin{tabular}{|c|}
\multicolumn{2}{|c|}{ Courtyard type -1 (1:1.5) } \\
November - April energy
\end{tabular}}} & & \multicolumn{4}{|c|}{ North } & \multicolumn{4}{|c|}{ East } & \multicolumn{4}{|c|}{ South } & \multicolumn{4}{|c|}{ West } \\
\hline & & & & Elec & Gas & $k W h / m 2$ & Decrease\% & Elec & Gas & $k W h / m 2$ & Decrease\% & Elec & Gas & kWh/m2 & Decrease\% & Elec & Gas & $k W h / m 2$ & Decrease\% \\
\hline \multirow{8}{*}{ Glazing } & \multirow{8}{*}{$\begin{array}{l}\text { Courtyard Trp Clr } \\
3 \mathrm{~mm} / 13 \mathrm{~mm} \text { Air } \\
\text { and sky light Dbl } \\
\text { LoE (e2=.1) Clr } \\
6 \mathrm{~mm} / 13 \mathrm{~mm} \text { Air }\end{array}$} & \multirow{2}{*}{$77 \%$} & Court & 2280.07 & 15505.94 & 109.5 & \begin{tabular}{|l|}
-16 \\
\end{tabular} & 2213.58 & 14310.22 & 101.73 & \begin{tabular}{|l|}
-10 \\
\end{tabular} & 2301.93 & 14083.08 & 100.87 & -6 & 2231.83 & 14547.12 & 103.3 & -11 \\
\hline & & & Atrium & 2276.84 & 12728.89 & 92.38 & & 2278.91 & 12608.6 & 91.65 & & 2278.58 & 13072.66 & 94.51 & & 2279.89 & 12674.26 & 92.06 & \\
\hline & & \multirow{2}{*}{$57 \%$} & Court & 2262.17 & 15147.28 & 107.18 & -14 & 2187.08 & 14156.96 & 100.62 & -9 & 2268.75 & 14140.71 & 101.02 & -6 & 2195.15 & 14389.06 & 102.1 & -10 \\
\hline & & & Atrium & 2284.7 & 12756.43 & 92.6 & & 2286.98 & 12639.09 & 91.89 & & 2286.7 & 13096.21 & 94.7 & & 2288.07 & 12703.92 & 92.3 & \\
\hline & & \multirow{2}{*}{$36 \%$} & Court & 2215.88 & 16654.76 & 116.17 & -15 & 2153.3 & 16570.27 & 115.27 & -15 & 2220.13 & 15876.87 & 111.41 & -9 & 2169.28 & 15561.35 & 109.16 & -9 \\
\hline & & & Atrium & 2309.61 & 13766.74 & 98.97 & & 2312.91 & 13677.33 & 98.44 & & 2312.08 & 14110.82 & 101.1 & & 2314.17 & 13743.69 & 98.86 & \\
\hline & & \multirow{2}{*}{$20 \%$} & Court & 2219.04 & 14633.09 & 103.75 & -10 & 2132.54 & 14180.89 & 100.43 & -8 & 2210.66 & 14686.42 & 104.02 & -9 & 2118.77 & 14419.4 & 101.81 & -9 \\
\hline & & & Atrium & 2298.24 & 12809.14 & 93.01 & & 2301.56 & 12697.16 & 92.34 & & 2300.84 & 13137.95 & 95.05 & & 2302.87 & 12760.64 & 92.74 & \\
\hline
\end{tabular}

Appendix VII Energy consumption by configuration -1, Option - 7

\begin{tabular}{|c|c|c|c|c|c|c|c|c|c|c|c|c|c|c|c|c|c|c|c|}
\hline \multirow{2}{*}{\multicolumn{3}{|c|}{\begin{tabular}{|c|}
\multicolumn{2}{|c}{ Courtyard type -1 (1:1.5) } \\
November - April energy
\end{tabular}}} & & \multicolumn{4}{|c|}{ North } & \multicolumn{4}{|c|}{ East } & \multicolumn{4}{|c|}{ South } & \multicolumn{4}{|c|}{ West } \\
\hline & & & & Elec & Gas & $k W h / m 2$ & Decrease\% & Elec & Gas & $k W h / m 2$ & Decrease \% & Elec & Gas & $\mathrm{kWh} / \mathrm{m} 2$ & Decrease\% & Elec & Gas & $\mathrm{kWh} / \mathrm{m} 2$ & Decrease\% \\
\hline \multirow{8}{*}{ Glazing } & \multirow{8}{*}{$\begin{array}{c}\text { Courtyard Trp LoE } \\
\text { (e2=e5=.1) Clr } \\
3 \mathrm{~mm} / 13 \mathrm{~mm} \mathrm{Air} \\
\text { and sky light Dbl } \\
\text { Clr } 6 \mathrm{~mm} / 13 \mathrm{~mm} \text { Air }\end{array}$} & \multirow{2}{*}{$77 \%$} & Court & 2187.48 & 14359.05 & 101.87 & \begin{tabular}{|l|}
-10 \\
\end{tabular} & 2142.61 & 13509.33 & 96.36 & -5 & 2198.99 & 13324.19 & 95.57 & -2 & 2153.73 & 13681.28 & 97.49 & -6 \\
\hline & & & Atrium & 2318.57 & 12613.21 & 91.92 & & 2322.81 & 12532.21 & 91.45 & & 2320.8 & 12863.45 & 93.48 & & 2324.38 & 12578.34 & 91.75 & \\
\hline & & & Court & 2173.88 & 14245.76 & 101.08 & -9 & 2124.43 & 13550.93 & 96.5 & -5 & 2175.8 & 13515.09 & 96.6 & -3 & 2128.46 & 13717.05 & 97.55 & -6 \\
\hline & & & Atrium & 2328.41 & 12633.05 & 92.11 & & 2332.69 & 12553.66 & 91.64 & & 2330.76 & 12880.89 & 93.65 & & 2334.33 & 12598.97 & 91.93 & \\
\hline & & & Court & 2158.5 & 16084.18 & 112.31 & -12 & 2135.19 & 16024.12 & 111.79 & -12 & 2160.8 & 15527.53 & 108.89 & -8 & 2137.42 & 15314.36 & 107.44 & -8 \\
\hline & & & Atrium & 2355.75 & 13626.55 & 98.39 & & 2360.75 & 13572.5 & 98.09 & & 2358.44 & 13880.49 & 99.97 & & 2362.42 & 13619.78 & 98.39 & \\
\hline & & \multirow{2}{*}{$20 \%$} & Court & 2146.55 & \begin{tabular}{|l|l|}
14178.8 \\
\end{tabular} & 100.5 & -8 & 2092.53 & 13855.71 & 98.18 & -6 & 2142.63 & 14209.25 & 100.67 & -7 & 2083 & 14020.97 & 99.14 & -7 \\
\hline & & & Atrium & 2346.31 & 12664.98 & 92.41 & & 2351.13 & 12590.29 & 91.98 & & 2349 & \begin{tabular}{|l|l}
12909.96 \\
\end{tabular} & 93.94 & & 2352.76 & 12635.74 & 92.27 & \\
\hline
\end{tabular}

Appendix VIII Energy consumption by configuration -1, Option - 8

\begin{tabular}{|c|c|c|c|c|c|c|c|c|c|c|c|c|c|c|c|c|c|c|c|}
\hline \multirow{2}{*}{\multicolumn{3}{|c|}{\begin{tabular}{|l|}
\multicolumn{2}{|c|}{ Courtyard type -1 (1:1.5) } \\
November - April energy
\end{tabular}}} & & \multicolumn{4}{|c|}{ North } & \multicolumn{4}{|c|}{ East } & \multicolumn{4}{|c|}{ South } & \multicolumn{4}{|c|}{ West } \\
\hline & & & & Elec & Gas & $k W h / m 2$ & Decrease\% & Elec & Gas & $k W h / m 2$ & Decrease\% & Elec & Gas & $k W h / m 2$ & Decrease\% & Elec & Gas & kWh/m2 & Decrease\% \\
\hline \multirow{8}{*}{ Glazing } & \multirow{8}{*}{$\begin{array}{c}\text { Courtyard Trp LoE } \\
\text { (e2=e5=.1) Clr } \\
3 \mathrm{~mm} / 13 \mathrm{~mm} \text { Air } \\
\text { and sky light Dbl } \\
\text { LoE (e2=.1) Clr } \\
6 \mathrm{~mm} / 13 \mathrm{~mm} \text { Air }\end{array}$} & \multirow{2}{*}{$77 \%$} & Court & 2187.48 & 14359.05 & \begin{tabular}{|l|l|}
101.87 \\
\end{tabular} & -12 & 2142.61 & 13509.33 & 96.36 & -7 & 2198.99 & 13324.19 & 95.57 & -4 & 2153.73 & \begin{tabular}{|l|}
13681.28 \\
\end{tabular} & \begin{tabular}{|c|}
97.49 \\
\end{tabular} & -8 \\
\hline & & & Atrium & 2274.52 & \begin{tabular}{|l|}
12341.3 \\
\end{tabular} & 89.98 & & 2278.64 & \begin{tabular}{|l|}
12259.82 \\
\end{tabular} & 89.5 & & 2276.8 & \begin{tabular}{|l|}
12592.6 \\
\end{tabular} & 91.54 & & 2280.23 & 12306.94 & 89.8 & \\
\hline & & \multirow{2}{*}{$57 \%$} & Court & 2173.88 & \begin{tabular}{|l|}
14245.76 \\
\end{tabular} & 101.08 & -11 & 2124.43 & \begin{tabular}{|l|}
13550.93 \\
\end{tabular} & 96.5 & -7 & 2175.8 & \begin{tabular}{|l|}
13515.09 \\
\end{tabular} & 96.6 & -5 & 2128.46 & 13717.05 & 97.55 & -8 \\
\hline & & & Atrium & 2282.98 & 12363.97 & 90.17 & & 2287.2 & 12283.87 & 89.7 & & 2285.44 & \begin{tabular}{|l|}
12612.68 \\
\end{tabular} & 91.72 & & 2288.87 & 12330.44 & 90 & \\
\hline & & \multirow{2}{*}{$36 \%$} & Court & 2158.5 & 16084.18 & 112.31 & -14 & 2135.19 & 16024.12 & 111.79 & -14 & 2160.8 & 15527.53 & 108.89 & -10 & 2137.42 & 15314.36 & 107.44 & -10 \\
\hline & & & Atrium & 2309.12 & 13360.02 & 96.46 & & 2314.1 & 13305.95 & 96.16 & & 2311.97 & \begin{tabular}{|l|}
13614.05 \\
\end{tabular} & 98.05 & & 2315.86 & 13353.26 & 96.46 & \\
\hline & & \multirow{2}{*}{$20 \%$} & Court & 2146.55 & \begin{tabular}{|l|}
14178.8 \\
\end{tabular} & 100.5 & -10 & 2092.53 & \begin{tabular}{|l|}
13855.71 \\
\end{tabular} & 98.18 & -8 & 2142.63 & 14209.25 & 100.67 & -9 & 2083 & \begin{tabular}{|l|}
14020.97 \\
\end{tabular} & 99.14 & -9 \\
\hline & & & Atrium & 2298.55 & 12399.22 & 90.48 & & 2303.42 & 12323.92 & 90.05 & & 2301.41 & 12643.95 & 92.01 & & 2305.15 & 12369.74 & 90.34 & \\
\hline
\end{tabular}

Appendix IX Energy consumption by configuration -2, Option - 1

\begin{tabular}{|c|c|c|c|c|c|c|c|c|c|c|c|c|c|c|c|c|c|c|c|}
\hline \multicolumn{4}{|c|}{ Courtyard type $-2(1: 1)$} & \multicolumn{4}{|c|}{ North } & \multicolumn{4}{|c|}{ East } & \multicolumn{4}{|c|}{ South } & \multicolumn{4}{|c|}{ West } \\
\hline \multicolumn{3}{|c|}{ November - April energy } & Option & Elec(kWh) & Gas(kWh) & $k W h / m 2$ & Decrease\% & Elec(kWh) & Gas(kWh) & $k W h / m 2$ & Decrease\% & Elec(kWh) & Gas(kWh) & $k W h / m 2$ & Decrease\% & Elec(kWh) & Gas(kWh) & $\mathrm{kWh} / \mathrm{m} 2$ & Decrease\% \\
\hline \multirow{8}{*}{ Glazing } & \multirow{8}{*}{$\begin{array}{c}\text { Courtyard Dble clr, } \\
6 \mathrm{~mm} / 13 \mathrm{~mm} \text { air } \\
\text { and sky light Dble } \\
\mathrm{clr}, 6 \mathrm{~mm} / 13 \mathrm{~mm} \text { air }\end{array}$} & \multirow{2}{*}{$73 \%$} & Court & 2329.95 & 17536.52 & 119.01 & \begin{tabular}{|l|}
-20 \\
\end{tabular} & 2256.16 & 16660.25 & 113.32 & -17 & 2350.38 & 16844.73 & 114.99 & -16 & 2249.67 & 16924.18 & \begin{tabular}{|l|}
114.86 \\
\end{tabular} & -18 \\
\hline & & & Atrium & 2252.32 & 13588.21 & 94.89 & & 2253.76 & 13471.27 & 94.2 & & 2253.83 & 13963.13 & 97.15 & & 2254.74 & 13543.02 & 94.63 & \\
\hline & & \multirow{2}{*}{$55 \%$} & Court & 2317.58 & 17046.87 & 116 & -18 & 2238.53 & 16287.28 & 110.98 & -15 & 2330.3 & 16588.68 & 113.33 & -14 & 2230.73 & 16546.82 & 112.48 & -16 \\
\hline & & & Atrium & 2257.87 & 13548.94 & 94.69 & & 2259.39 & 13434.53 & 94.01 & & 2259.57 & 13919.41 & 96.92 & & 2260.47 & 13505.14 & 94.44 & \\
\hline & & \multirow{2}{*}{$36 \%$} & Court & 2290.8 & 16648.45 & 113.45 & -16 & 2212.08 & 16135.82 & 109.91 & -14 & 2295.93 & 16623.26 & 113.33 & -14 & 2199.2 & 16385.62 & 111.33 & -15 \\
\hline & & & Atrium & 2263.82 & 13576.67 & 94.89 & & 2265.79 & 13465.9 & 94.24 & & 2265.8 & 13942.92 & 97.1 & & 2267.02 & 13535.58 & 94.66 & \\
\hline & & \multirow{2}{*}{$23 \%$} & Court & 2276.58 & 16251.62 & 110.99 & -15 & 2197.92 & 15880.4 & 108.3 & -13 & 2275.88 & 16453.34 & 112.19 & -14 & 2182.41 & 16125.17 & 109.67 & -14 \\
\hline & & & Atrium & \begin{tabular}{|l|}
2270.23 \\
\end{tabular} & 13543.06 & 94.73 & & 2272.57 & 13435.41 & 94.1 & & 2272.45 & 13904.96 & 96.91 & & 2273.79 & 13504.35 & 94.52 & \\
\hline
\end{tabular}

Appendix X Energy consumption by configuration -2, Option - 2

\begin{tabular}{|c|c|c|c|c|c|c|c|c|c|c|c|c|c|c|c|c|c|c|c|}
\hline \multicolumn{4}{|c|}{ Courtyard type -2 (1:1) } & \multicolumn{4}{|c|}{ North } & \multicolumn{4}{|c|}{ East } & \multicolumn{4}{|c|}{ South } & \multicolumn{4}{|c|}{ West } \\
\hline Novembe & $r$-April energy & & Option & Elec & Gas & $k W h / m 2$ & Decrease\% & Elec & Gas & $k W h / m 2$ & Decrease\% & Elec & Gas & $k W h / m 2$ & Decrease\% & Elec & Gas & kWh/m2 & Decrease\% \\
\hline \multirow{8}{*}{ Glazing } & \multirow{8}{*}{$\begin{array}{l}\text { Courtyard Dble clr, } \\
6 \mathrm{~mm} / 13 \mathrm{~mm} \text { air } \\
\text { and sky light Dbl } \\
\text { LoE (e2=.1) Clr } \\
6 \mathrm{~mm} / 13 \mathrm{~mm} \text { Air }\end{array}$} & \multirow{2}{*}{$73 \%$} & Court & 2329.95 & 17536.52 & 119.01 & -21 & 2256.16 & 16660.25 & 113.32 & -18 & 2350.38 & 16844.73 & 114.99 & -17 & 2249.67 & 16924.18 & 114.86 & -19 \\
\hline & & & Atrium & 2224.09 & 13399.01 & 93.59 & & 2225.33 & 13283.18 & 92.9 & & 2225.63 & 13775.94 & 95.86 & & 2226.31 & 13355.31 & 93.34 & \\
\hline & & \multirow[b]{2}{*}{$55 \%$} & Court & 2317.58 & 17046.87 & 116 & -19 & 2238.53 & 16287.28 & 110.98 & -16 & 2330.3 & 16588.68 & 113.33 & -16 & 2230.73 & 16546.82 & 112.48 & -17 \\
\hline & & & Atrium & 2228.11 & 13374.75 & 93.47 & & 2229.4 & 13260.96 & 92.79 & & 2229.84 & \begin{tabular}{|c|}
13747.16 \\
\end{tabular} & 95.71 & & 2230.51 & 13332.36 & 93.23 & \\
\hline & & \multirow{2}{*}{$36 \%$} & Court & 2290.8 & 16648.45 & 113.45 & -16 & 2212.08 & 16135.82 & 109.91 & -14 & 2295.93 & 16623.26 & 113.33 & -14 & 2199.2 & 16385.62 & 111.33 & -15 \\
\hline & & & Atrium & 2263.82 & 13576.67 & 94.89 & & 2265.79 & 13465.9 & 94.24 & & 2265.8 & 13942.92 & \begin{tabular}{|l|}
97.1 \\
\end{tabular} & & 2267.02 & 13535.58 & 94.66 & \\
\hline & & \multirow{2}{*}{$23 \%$} & Court & 2276.58 & 16251.62 & 110.99 & -16 & 2197.92 & 15880.4 & 108.3 & -14 & 2275.88 & 16453.34 & 112.19 & -15 & 2182.41 & 16125.17 & 109.67 & -15 \\
\hline & & & Atrium & 2239.52 & \begin{tabular}{|l|l}
13364.6 \\
\end{tabular} & 93.47 & & 2241.74 & 13256.07 & 92.84 & & 2241.79 & 13726.84 & 95.66 & & 2243.1 & 13325.83 & 93.26 & \\
\hline
\end{tabular}


Appendix XI Energy consumption by configuration -2, Option - 3

\begin{tabular}{|c|c|c|c|c|c|c|c|c|c|c|c|c|c|c|c|c|c|c|c|}
\hline \multicolumn{4}{|c|}{ Courtyard type -2 (1:1) } & \multicolumn{4}{|c|}{ North } & \multicolumn{4}{|c|}{ East } & \multicolumn{4}{|c|}{ South } & \multicolumn{4}{|c|}{ West } \\
\hline Novembe & r - April energy & & Option & Elec & Gas & $k W h / m 2$ & Decrease\% & Elec & Gas & kWh/m2 & Decrease\% & Elec & Gas & kWh/m2 & Decrease\% & \begin{tabular}{|l} 
Elec \\
\end{tabular} & Gas & $\mathrm{kWh} / \mathrm{m} 2$ & Decrease $\%$ \\
\hline \multirow{8}{*}{ Glazing } & \multirow{8}{*}{$\begin{array}{c}\text { Courtyard Dbl LoE } \\
\text { (e2=.1) Clr } \\
6 \mathrm{~mm} / 13 \mathrm{~mm} \text { Air } \\
\text { and sky light Dble } \\
\text { clr, } 6 \mathrm{~mm} / 13 \mathrm{~mm} \text { air }\end{array}$} & \multirow{2}{*}{$73 \%$} & Court & 2265.77 & 15808.16 & 108.27 & -16 & 2205.87 & 15103.85 & 103.69 & -13 & 2278.3 & 15246.15 & \begin{tabular}{|l|l|}
104.98 \\
\end{tabular} & -12 & 2200.44 & 15314.01 & 104.92 & -14 \\
\hline & & & Atrium & 2243.02 & 12914.38 & 90.8 & & 2245.38 & 12822.51 & 90.26 & & 2244.8 & 13217.22 & 92.62 & & 2246.68 & 12881.06 & 90.62 & \\
\hline & & \multirow{2}{*}{$55 \%$} & Court & 2256.6 & 15536.24 & 106.59 & -15 & 2192.47 & 14928.19 & 102.56 & -12 & 2262.75 & 15165.73 & 104.4 & -12 & 2186.09 & 15132.91 & 103.75 & -13 \\
\hline & & & Atrium & 2248.8 & 12873.45 & 90.59 & & 2251.26 & 12783.59 & 90.06 & & 2250.73 & 13173.05 & 92.39 & & 2252.62 & 12841.23 & 90.42 & \\
\hline & & \multirow{2}{*}{$36 \%$} & Court & 2234.9 & 15373.7 & 105.48 & -14 & 2172.71 & 14960.39 & 102.63 & -12 & 2236.64 & 15355.11 & 105.38 & -12 & 2161.99 & 15155.31 & 103.74 & -13 \\
\hline & & & Atrium & 2255.26 & 12898.41 & 90.78 & & 2258.14 & 12811.05 & 90.27 & & 2257.45 & 13193.87 & 92.56 & & 2259.57 & 12868.25 & 90.62 & \\
\hline & & \multirow{2}{*}{$23 \%$} & Court & 2224.18 & 15176.82 & 104.24 & -13 & 2162.35 & 14872.83 & 102.05 & -12 & 2221.28 & 15341.69 & 105.21 & -12 & 2150.05 & 15065.08 & 103.12 & -12 \\
\hline & & & Atrium & 2262.03 & 12860.27 & 90.59 & & 2265.15 & 12775.31 & 90.1 & & 2264.39 & 13152.99 & 92.36 & & 2266.65 & 12831.97 & 90.45 & \\
\hline
\end{tabular}

Appendix XII Energy consumption by configuration -2, Option - 4

\begin{tabular}{|c|c|c|c|c|c|c|c|c|c|c|c|c|c|c|c|c|c|c|c|}
\hline \multicolumn{4}{|c|}{ Courtyard type $-2(1: 1)$} & \multicolumn{4}{|c|}{ North } & \multicolumn{4}{|c|}{ East } & \multicolumn{4}{|c|}{ South } & \multicolumn{4}{|c|}{ West } \\
\hline \multicolumn{3}{|c|}{ November - April energy } & Option & Elec & Gas & $\mathrm{kWh} / \mathrm{m} 2$ & Decrease\% & Elec & Gas & kWh/m2 & Decrease\% & Elec & Gas & $\mathrm{kWh} / \mathrm{m} 2$ & Decrease\% & Elec & Gas & $\mathrm{kWh} / \mathrm{m} 2$ & Decrease\% \\
\hline \multirow{8}{*}{ Glazing } & \multirow{8}{*}{$\begin{array}{c}\text { Courtyard Dbl LoE } \\
(\mathrm{e} 2=.1) \mathrm{Clr} \\
6 \mathrm{~mm} / 13 \mathrm{~mm} \text { Air } \\
\text { and sky light Dbl } \\
\text { LoE (e2=.1) Clr } \\
6 \mathrm{~mm} / 13 \mathrm{~mm} \text { Air }\end{array}$} & \multirow{2}{*}{$73 \%$} & Court & 2265.77 & 15808.16 & 108.27 & \begin{tabular}{|l|}
-17 \\
\end{tabular} & 2205.87 & 15103.85 & \begin{tabular}{|l|}
103.69 \\
\end{tabular} & \begin{tabular}{|l|}
-14 \\
\end{tabular} & 2278.3 & 15246.15 & \begin{tabular}{|l|}
104.98 \\
\end{tabular} & \begin{tabular}{|l|}
-13 \\
\end{tabular} & 2200.44 & 15314.01 & 104.92 & -15 \\
\hline & & & Atrium & 2214.51 & 12728.54 & 89.51 & & 2216.7 & 12636.86 & 88.98 & & 2216.34 & 13032.62 & 91.35 & & 2218.02 & 12695.81 & 89.34 & \\
\hline & & \multirow{2}{*}{$55 \%$} & Court & 2256.6 & 15536.24 & 106.59 & -16 & 2192.47 & 14928.19 & 102.56 & -13 & 2262.75 & 15165.73 & 104.4 & -13 & 2186.09 & 15132.91 & 103.75 & -14 \\
\hline & & & Atrium & 2218.76 & 12702.75 & 89.39 & & 2221.06 & 12612.94 & 88.86 & & 2220.77 & 13003.11 & 91.2 & & 2222.46 & 12670.37 & 89.21 & \\
\hline & & \multirow{2}{*}{$36 \%$} & Court & 2234.9 & \begin{tabular}{|l|}
15373.7 \\
\end{tabular} & 105.48 & -14 & 2172.71 & 14960.39 & 102.63 & -12 & 2236.64 & 15355.11 & 105.38 & -12 & 2161.99 & 15155.31 & 103.74 & -13 \\
\hline & & & Atrium & 2255.26 & 12898.41 & 90.78 & & 2258.14 & 12811.05 & 90.27 & & 2257.45 & 13193.87 & 92.56 & & 2259.57 & 12868.25 & 90.62 & \\
\hline & & \multirow{2}{*}{$23 \%$} & Court & 2224.18 & 15176.82 & 104.24 & -14 & 2162.35 & 14872.83 & 102.05 & -13 & 2221.28 & 15341.69 & 105.21 & -13 & 2150.05 & 15065.08 & 103.12 & -14 \\
\hline & & & Atrium & 2231.18 & 12681.63 & 89.33 & & 2234.27 & 12596.54 & 88.84 & & 2233.6 & 12974.46 & 91.1 & & 2235.81 & 12653.31 & 89.19 & \\
\hline
\end{tabular}

Appendix XIII Energy consumption by configuration -2, Option - 5

\begin{tabular}{|c|c|c|c|c|c|c|c|c|c|c|c|c|c|c|c|c|c|c|c|}
\hline \multicolumn{4}{|c|}{ Courtyard type -2 (1:1) } & \multicolumn{4}{|c|}{ North } & \multicolumn{4}{|c|}{ East } & \multicolumn{4}{|c|}{ South } & \multicolumn{4}{|c|}{ West } \\
\hline \multicolumn{3}{|c|}{ November - April energy } & Option & Elec & Gas & $k W h / m 2$ & Decrease\% & Elec & Gas & $k W h / m 2$ & Decrease\% & Elec & Gas & $\mathrm{kWh} / \mathrm{m} 2$ & Decrease\% & Elec & Gas & $\mathrm{kWh} / \mathrm{m} 2$ & Decrease\% \\
\hline \multirow{8}{*}{ Glazing } & \multirow{8}{*}{$\begin{array}{l}\text { Courtyard Trp Clr } \\
3 \mathrm{~mm} / 13 \mathrm{~mm} \text { Air } \\
\text { and sky light Dbl } \\
\text { Clr } 6 \mathrm{~mm} / 13 \mathrm{~mm} \text { Air }\end{array}$} & \multirow{2}{*}{$73 \%$} & Court & 2316.69 & 15493.93 & 106.69 & -16 & 2235.3 & 14652 & 101.16 & -12 & 2327.68 & 14848.81 & 102.89 & \begin{tabular}{|c|}
-10 \\
\end{tabular} & 2228.9 & \begin{tabular}{|l|l}
14891.78 \\
\end{tabular} & 102.56 & -12 \\
\hline & & & Atrium & 2247.56 & 12786.54 & 90.06 & & 2249.23 & 12666.07 & 89.35 & & 2249.27 & 13136.1 & 92.16 & & 2250.53 & 12733.89 & 89.76 & \\
\hline & & \multirow{2}{*}{$55 \%$} & Court & 2306.32 & 15202.48 & 104.88 & -14 & 2219.54 & 14472.04 & 99.99 & -11 & 2309.02 & 14781.91 & 102.38 & -10 & 2212.66 & 14705.58 & 101.35 & -12 \\
\hline & & & Atrium & 2253.06 & 12749.93 & 89.87 & & 2254.88 & 12631.49 & 89.17 & & 2255 & 13094.84 & 91.95 & & 2256.25 & 12698.18 & 89.58 & \\
\hline & & \multirow{2}{*}{$36 \%$} & Court & 2281.58 & 15043.88 & 103.79 & -13 & 2196.29 & 14542.28 & 100.27 & -11 & 2278.44 & 15031.26 & 103.69 & -11 & 2183.51 & 14774.84 & 101.59 & -12 \\
\hline & & & Atrium & 2259.02 & 12779.03 & 90.08 & & 2261.35 & 12663.28 & 89.4 & & 2261.24 & 13118.23 & 92.13 & & 2262.85 & 12728.84 & 89.81 & \\
\hline & & \multirow{2}{*}{$23 \%$} & Court & 2267.74 & 14839.92 & 102.48 & -12 & 2183.96 & 14468.65 & 99.76 & -11 & 2260.19 & 15038.66 & 103.63 & -11 & 2169.83 & \begin{tabular}{|l|}
14690.42 \\
\end{tabular} & 101 & -11 \\
\hline & & & Atrium & 2265.41 & 12744.53 & 89.91 & & 2268.14 & 12632.02 & 89.26 & & 2267.82 & 13080.37 & 91.94 & & 2269.63 & 12696.83 & 89.65 & \\
\hline
\end{tabular}

Appendix XIV Energy consumption by configuration -2, Option - 6

\begin{tabular}{|c|c|c|c|c|c|c|c|c|c|c|c|c|c|c|c|c|c|c|c|}
\hline \multicolumn{4}{|c|}{ Courtyard type -2 (1:1) } & \multicolumn{4}{|c|}{ North } & \multicolumn{4}{|c|}{ East } & \multicolumn{4}{|c|}{ South } & \multicolumn{4}{|c|}{ West } \\
\hline Novembe & \multirow{9}{*}{$\begin{array}{c}\text { Courtyard Trp Clr } \\
3 \mathrm{~mm} / 13 \mathrm{~mm} \text { Air } \\
\text { and sky light Dbl } \\
\text { LoE (e2=.1) Clr } \\
6 \mathrm{~mm} / 13 \mathrm{~mm} \text { Air }\end{array}$} & & Option & Elec & Gas & $\mathrm{kWh} / \mathrm{m} 2$ & Decrease\% & Elec & Gas & kWh/m2 & Decrease\% & Elec & Gas & kWh/m2 & Decrease\% & Elec & Gas & $\mathrm{kWh} / \mathrm{m} 2$ & Decrease\% \\
\hline \multirow{8}{*}{ Glazing } & & \multirow{2}{*}{$73 \%$} & Court & 2316.69 & 15493.93 & 106.69 & \begin{tabular}{|l|}
-17 \\
\end{tabular} & 2235.3 & 14652 & 101.16 & \begin{tabular}{|l|}
-13 \\
\end{tabular} & 2327.68 & 14848.81 & 102.89 & \begin{tabular}{|l|}
-12 \\
\end{tabular} & 2228.9 & \begin{tabular}{|c|}
14891.78 \\
\end{tabular} & 102.56 & -14 \\
\hline & & & Atrium & 2219.14 & 12598.94 & 88.77 & & 2220.62 & 12478.76 & 88.05 & & 2220.95 & 12950.13 & 90.88 & & 2221.94 & 12547.19 & 88.47 & \\
\hline & & \multirow{2}{*}{$55 \%$} & Court & 2306.32 & 15202.48 & 104.88 & -15 & 2219.54 & 14472.04 & 99.99 & -12 & 2309.02 & 14781.91 & 102.38 & -11 & 2212.66 & 14705.58 & 101.35 & -13 \\
\hline & & & Atrium & 2223.13 & 12576.94 & 88.66 & & 2224.71 & 12458.97 & 87.96 & & 2225.11 & 12922.98 & 90.74 & & 2226.11 & \begin{tabular}{|c|}
12525.87 \\
\end{tabular} & 88.37 & \\
\hline & & \multirow{2}{*}{$36 \%$} & Court & 2281.58 & 15043.88 & 103.79 & -13 & 2196.29 & 14542.28 & 100.27 & -11 & 2278.44 & 15031.26 & 103.69 & -11 & 2183.51 & \begin{tabular}{|l|}
14774.84 \\
\end{tabular} & 101.59 & -12 \\
\hline & & & Atrium & 2259.02 & 12779.03 & 90.08 & & 2261.35 & 12663.28 & 89.4 & & 2261.24 & 13118.23 & 92.13 & & 2262.85 & 12728.84 & 89.81 & \\
\hline & & \multirow{2}{*}{$23 \%$} & Court & 2267.74 & 14839.92 & 102.48 & -13 & 2183.96 & 14468.65 & 99.76 & -12 & 2260.19 & 15038.66 & 103.63 & -12 & 2169.83 & 14690.42 & 101 & -12 \\
\hline & & & Atrium & 2234.58 & 12565.91 & 88.66 & & 2237.2 & 12453.48 & 88 & & 2237.05 & 12901.96 & 90.69 & & 2238.81 & 12518.39 & 88.4 & \\
\hline
\end{tabular}

Appendix XV Energy consumption by configuration -2, Option - 7

\begin{tabular}{|c|c|c|c|c|c|c|c|c|c|c|c|c|c|c|c|c|c|c|c|}
\hline \multicolumn{4}{|c|}{ Courtyard type -2 (1:1) } & \multicolumn{4}{|c|}{ North } & \multicolumn{4}{|c|}{ East } & \multicolumn{4}{|c|}{ South } & \multicolumn{4}{|c|}{ West } \\
\hline Novembe & - April energy & & Option & Elec & Gas & $k W h / m 2$ & Decrease\% & Elec & Gas & kWh/m2 & Decrease\% & Elec & Gas & $k W h / m 2$ & Decrease\% & Elec & Gas & kWh/m2 & Decrease\% \\
\hline \multirow{8}{*}{ Glazing } & \multirow{8}{*}{$\begin{array}{c}\text { Courtyard Trp LoE } \\
\text { (e2=e5=.1) Clr } \\
3 \mathrm{~mm} / 13 \mathrm{~mm} \text { Air } \\
\text { and sky light Dbl } \\
\text { Clr } 6 \mathrm{~mm} / 13 \mathrm{~mm} \text { Air }\end{array}$} & \multirow{2}{*}{$73 \%$} & Court & 2228.65 & 14514.86 & 100.3 & -13 & 2175.98 & 13924.88 & 96.45 & -10 & 2233.72 & 14049.53 & 97.54 & -8 & 2170.69 & 14091.02 & \begin{tabular}{|l|}
97.41 \\
\end{tabular} & -10 \\
\hline & & & Atrium & 2245.88 & 12401.51 & 87.74 & & 2249.59 & 12321.12 & 87.28 & & 2248.21 & 12658.34 & 89.3 & & 2251.56 & 12370.22 & 87.59 & \\
\hline & & \multirow{2}{*}{$55 \%$} & Court & \begin{tabular}{|l|}
2221.61 \\
\end{tabular} & 14411.76 & 99.64 & -12 & 2165.5 & 13898.86 & 96.23 & -10 & 2221.82 & 14107.16 & 97.82 & -9 & 2160.17 & 14061.11 & 97.17 & -10 \\
\hline & & & Atrium & 2251.84 & 12358.86 & 87.52 & & 2255.63 & 12279.93 & 87.07 & & 2254.25 & 12612.84 & 89.06 & & 2257.6 & 12328.42 & 87.38 & \\
\hline & & & Court & 2205.24 & 14427.05 & 99.63 & -12 & 2151.47 & 14072.39 & 97.19 & -10 & 2202.53 & $\mid 14416.1$ & 99.55 & -10 & 2142.87 & 14230.97 & 98.09 & -11 \\
\hline & & & Atrium & 2258.75 & 12378.75 & 87.68 & & 2262.87 & 12301.54 & 87.25 & & 2261.32 & 12629.67 & 89.2 & & 2264.87 & 12349.24 & 87.54 & \\
\hline & & \multirow{2}{*}{$23 \%$} & Court & 2196.69 & 14371.15 & 99.25 & -12 & 2144.99 & 14109.12 & 97.37 & -11 & 2193.52 & 14508.97 & 100.05 & -11 & 2135.19 & 14263.07 & 98.23 & -11 \\
\hline & & & Atrium & 2265.78 & 12336.53 & 87.47 & & 2270.14 & 12261.51 & 87.05 & & \begin{tabular}{|l}
2268.48 \\
\end{tabular} & \begin{tabular}{|l}
12585.93 \\
\end{tabular} & 88.98 & & \begin{tabular}{|l|}
2272.12 \\
\end{tabular} & \begin{tabular}{|l|l}
12308.73 \\
\end{tabular} & 87.34 & \\
\hline
\end{tabular}


Appendix XVI Energy consumption by configuration -2, Option - 8

\begin{tabular}{|c|c|c|c|c|c|c|c|c|c|c|c|c|c|c|c|c|c|c|c|}
\hline \multicolumn{4}{|c|}{ Courtyard type -2 (1:1) } & \multicolumn{4}{|c|}{ North } & \multicolumn{4}{|c|}{ East } & \multicolumn{4}{|c|}{ South } & \multicolumn{4}{|c|}{ West } \\
\hline Novembe & - April energy & & Option & Elec & Gas & kWh/m2 & Decrease\% & Elec & Gas & $\mathrm{kWh} / \mathrm{m} 2$ & Decrease\% & Elec & Gas & $\mathrm{kWh} / \mathrm{m} 2$ & Decrease $\%$ & Elec & Gas & kWh/m2 & Decrease\% \\
\hline \multirow{8}{*}{ Glazing } & \multirow{8}{*}{$\begin{array}{c}\text { Courtyard Trp LoE } \\
\text { (e2=e5=.1) Clr } \\
3 \mathrm{~mm} / 13 \mathrm{~mm} \text { Air } \\
\text { and sky light Dbl } \\
\text { LoE (e2=.1) Clr } \\
6 \mathrm{~mm} / 13 \mathrm{~mm} \text { Air }\end{array}$} & \multirow{2}{*}{$73 \%$} & Court & 2228.65 & 14514.86 & 100.3 & \begin{tabular}{|l|}
-14 \\
\end{tabular} & 2175.98 & 13924.88 & 96.45 & -11 & 2233.72 & 14049.53 & 97.54 & \begin{tabular}{|l|}
-10 \\
\end{tabular} & 2170.69 & 14091.02 & \begin{tabular}{|l|}
97.41 \\
\end{tabular} & -11 \\
\hline & & & Atrium & 2216.92 & 12218.21 & 86.47 & & 2220.52 & 12138.26 & 86.01 & & 2219.3 & 12475.87 & 88.03 & & 2222.49 & 12187.6 & 86.32 & \\
\hline & & \multirow{2}{*}{$55 \%$} & Court & 2221.61 & 14411.76 & 99.64 & -13 & 2165.5 & 13898.86 & 96.23 & -11 & 2221.82 & 14107.16 & 97.82 & -10 & 2160.17 & 14061.11 & 97.17 & -11 \\
\hline & & & Atrium & 2221.48 & 12189.44 & 86.33 & & 2225.21 & 12110.47 & 85.88 & & 2224 & 12444.14 & 87.87 & & 2227.19 & 12159.34 & 86.18 & \\
\hline & & \multirow{2}{*}{$36 \%$} & Court & 2205.24 & 14427.05 & 99.63 & -12 & 2151.47 & 14072.39 & 97.19 & -10 & 2202.53 & 14416.1 & 99.55 & -10 & 2142.87 & 14230.97 & 98.09 & -11 \\
\hline & & & Atrium & 2258.75 & 12378.75 & 87.68 & & 2262.87 & 12301.54 & 87.25 & & 2261.32 & 12629.67 & 89.2 & & 2264.87 & 12349.24 & 87.54 & \\
\hline & & \multirow{2}{*}{$23 \%$} & Court & 2196.69 & 14371.15 & 99.25 & -13 & 2144.99 & 14109.12 & 97.37 & -12 & 2193.52 & 14508.97 & 100.05 & -12 & \begin{tabular}{|l}
2135.19 \\
\end{tabular} & 14263.07 & 98.23 & -12 \\
\hline & & & Atrium & 2234.83 & \begin{tabular}{|l}
12158.35 \\
\end{tabular} & 86.22 & & 2239.18 & \begin{tabular}{|l|}
12083.12 \\
\end{tabular} & 85.8 & & 2237.65 & \begin{tabular}{|l|}
12407.95 \\
\end{tabular} & 87.73 & & 2241.23 & 12130.49 & 86.09 & \\
\hline
\end{tabular}

Appendix XVII Daylighting condition of configuration -1, Option - 1

\begin{tabular}{|c|c|c|c|c|c|c|c|c|c|c|c|}
\hline \multicolumn{4}{|c|}{ Courtyard type -1 (1:1.5) } & \multicolumn{2}{|l|}{ North } & \multicolumn{2}{|l|}{ East } & \multicolumn{2}{|l|}{ South } & \multicolumn{2}{|l|}{ West } \\
\hline \multicolumn{3}{|c|}{ November - April energy } & Daylight & Above threshold \% & Decrease \% & Above threshold \% & Decrease \% & Above threshold \% & Decrease \% & Above threshold \% & Decrease \% \\
\hline \multirow{8}{*}{ Glazing } & \multirow{8}{*}{$\begin{array}{c}\text { Courtyard Dble clr, } \\
6 \mathrm{~mm} / 13 \mathrm{~mm} \text { air } \\
\text { and sky light Dble } \\
\text { clr, } 6 \mathrm{~mm} / 13 \mathrm{~mm} \text { air }\end{array}$} & \multirow{2}{*}{$77 \%$} & Court & 99.694 & -0.6 & 99.796 & -0.3 & 99.898 & -0.8 & 99.898 & -0.7 \\
\hline & & & Atrium & 99.081 & & 99.489 & & 99.081 & & 99.183 & \\
\hline & & \multirow{2}{*}{$57 \%$} & Court & 99.796 & -1.1 & 99.898 & -1.2 & 99.694 & -0.6 & 99.796 & -1.0 \\
\hline & & & Atrium & 98.672 & & 98.672 & & 99.081 & & 98.774 & \\
\hline & & \multirow{2}{*}{$36 \%$} & Court & 86.005 & -5.9 & 86.209 & -6.2 & 86.107 & -5.9 & 86.618 & -7.8 \\
\hline & & & Atrium & 80.898 & & 80.898 & & 81 & & 79.876 & \\
\hline & & \multirow{2}{*}{$20 \%$} & Court & 72.215 & -6.2 & 72.419 & -5.8 & 72.113 & -7.2 & 72.93 & -7.0 \\
\hline & & & Atrium & 67.72 & & 68.231 & & 66.903 & & 67.822 & \\
\hline
\end{tabular}

Appendix XVIII Daylighting condition of configuration -1, Option - 2

\begin{tabular}{|c|c|c|c|c|c|c|c|c|c|c|c|}
\hline \multicolumn{4}{|c|}{ Courtyard type -1 (1:1.5) } & \multicolumn{2}{|l|}{ North } & \multicolumn{2}{|l|}{ East } & \multicolumn{2}{|l|}{ South } & \multicolumn{2}{|l|}{ West } \\
\hline \multicolumn{3}{|c|}{ November - February energy } & Daylight & Above threshold \% & Decrease \% & Above threshold \% & Decrease \% & Above threshold \% & Decrease \% & Above threshold \% & Decrease \% \\
\hline \multirow{8}{*}{ Glazing } & \multirow{8}{*}{$\begin{array}{l}\text { Courtyard Dble clr, } \\
6 \mathrm{~mm} / 13 \mathrm{~mm} \text { air } \\
\text { and sky light Dbl } \\
\text { LoE (e2=.1) Clr } \\
6 \mathrm{~mm} / 13 \mathrm{~mm} \text { Air }\end{array}$} & \multirow{2}{*}{$77 \%$} & Court & 99.898 & -0.9 & 99.898 & -0.9 & 99.796 & -1.0 & 99.898 & -0.9 \\
\hline & & & Atrium & 98.978 & & 98.978 & & 98.774 & & 98.978 & \\
\hline & & \multirow{2}{*}{$57 \%$} & Court & 99.898 & -1.8 & 99.796 & -1.4 & 99.796 & -1.3 & 99.591 & -1.6 \\
\hline & & & Atrium & 98.059 & & 98.366 & & 98.468 & & 97.957 & \\
\hline & & \multirow{2}{*}{$36 \%$} & Court & 85.086 & -6.0 & 86.209 & -6.8 & 86.414 & -7.3 & 86.312 & -6.7 \\
\hline & & & Atrium & 79.978 & & 80.387 & & 80.08 & & 80.489 & \\
\hline & & \multirow{2}{*}{$20 \%$} & Court & 73.032 & -7.8 & 72.215 & -5.4 & 72.215 & -7.1 & 72.01 & -6.4 \\
\hline & & & Atrium & 67.311 & & 68.333 & & 67.107 & & 67.414 & \\
\hline
\end{tabular}

Appendix XIX Daylighting condition of configuration -1, Option - 3

\begin{tabular}{|c|c|c|c|c|c|c|c|c|c|c|c|}
\hline \multicolumn{4}{|c|}{ Courtyard type -1 (1:1.5) } & \multicolumn{2}{|l|}{ North } & \multicolumn{2}{|l|}{ East } & \multicolumn{2}{|c|}{ South } & \multicolumn{2}{|l|}{ West } \\
\hline \multicolumn{3}{|c|}{ November - February energy } & Daylight & Above threshold \% & Decrease \% & Above threshold \% & Decrease \% & Above threshold \% & Decrease \% & Above threshold \% & Decrease \% \\
\hline \multirow{8}{*}{ Glazing } & \multirow{8}{*}{$\begin{array}{c}\text { Courtyard Dbl LoE } \\
(\mathrm{e} 2=.1) \mathrm{Clr} \\
6 \mathrm{~mm} / 13 \mathrm{~mm} \text { Air } \\
\text { and sky light Dbl } \\
\text { Clr } 6 \mathrm{~mm} / 13 \mathrm{~mm} \text { Air }\end{array}$} & \multirow{2}{*}{$77 \%$} & \begin{tabular}{|l|} 
Court \\
\end{tabular} & 99.898 & -1.1 & \begin{tabular}{|l|}
99.796 \\
\end{tabular} & $\mid-0.9$ & 99.898 & -0.6 & \begin{tabular}{|l|}
99.898 \\
\end{tabular} & -0.9 \\
\hline & & & Atrium & 98.774 & & 98.876 & & 99.285 & & 98.978 & \\
\hline & & \multirow{2}{*}{$57 \%$} & Court & 99.694 & -1.3 & 99.694 & -1.9 & 99.591 & -1.2 & 99.694 & -1.4 \\
\hline & & & Atrium & 98.366 & & 97.753 & & 98.366 & & 98.263 & \\
\hline & & \multirow{2}{*}{$36 \%$} & Court & 85.086 & -6.8 & 84.882 & -7.6 & 84.473 & -6.5 & 85.188 & -7.2 \\
\hline & & & Atrium & 79.263 & & 78.446 & & 78.957 & & 79.059 & \\
\hline & & \multirow{2}{*}{$20 \%$} & Court & 70.989 & -7.1 & 71.704 & -7.4 & 72.113 & -8.6 & 71.091 & -7.6 \\
\hline & & & Atrium & 65.983 & & 66.392 & & 65.881 & & 65.677 & \\
\hline
\end{tabular}

Appendix XX Daylighting condition of configuration -1, Option - 4

\begin{tabular}{|c|c|c|c|c|c|c|c|c|c|c|c|}
\hline \multicolumn{3}{|c|}{ Courtyard type -1 (1:1.5) } & & \multicolumn{2}{|l|}{ North } & \multicolumn{2}{|l|}{ East } & \multicolumn{2}{|l|}{ South } & \multicolumn{2}{|l|}{ West } \\
\hline \multicolumn{3}{|c|}{ November -February energy } & Daylight & Above threshold \% & Decrease \% & Above threshold \% & Decrease \% & Above threshold \% & Decrease \% & Above threshold \% & Decrease \% \\
\hline \multirow{8}{*}{ Glazing } & \multirow{8}{*}{$\begin{array}{c}\text { Courtyard Dbl LoE } \\
\text { (e2=.1) Clr } \\
6 \mathrm{~mm} / 13 \mathrm{~mm} \text { Air } \\
\text { and sky light Dbl } \\
\text { LoE (e2=.1) Clr } \\
6 \mathrm{~mm} / 13 \mathrm{~mm} \text { Air }\end{array}$} & \multirow{2}{*}{$77 \%$} & Court & 99.796 & -1.6 & 99.898 & -1.9 & 99.796 & -1.9 & 99.796 & -1.7 \\
\hline & & & Atrium & 98.161 & & 97.957 & & 97.855 & & 98.059 & \\
\hline & & \multirow{2}{*}{$57 \%$} & Court & 99.796 & -2.3 & 99.694 & -2.3 & 99.694 & -2.9 & 99.591 & -2.4 \\
\hline & & & Atrium & 97.548 & & 97.446 & & 96.833 & & 97.242 & \\
\hline & & \multirow{2}{*}{$36 \%$} & Court & 84.064 & -7.2 & 83.247 & -6.1 & 85.494 & -9.0 & 83.962 & -6.6 \\
\hline & & & Atrium & 78.037 & & 78.139 & & 77.833 & & 78.446 & \\
\hline & & \multirow{2}{*}{$20 \%$} & Court & 70.785 & -6.4 & 71.704 & -8.0 & 69.354 & -4.4 & 71.5 & -8.1 \\
\hline & & & Atrium & 66.29 & & 65.983 & & 66.29 & & 65.677 & \\
\hline
\end{tabular}


Appendix XXI Daylighting condition of configuration -1, Option - 5

\begin{tabular}{|c|c|c|c|c|c|c|c|c|c|c|c|}
\hline \multirow{2}{*}{\multicolumn{3}{|c|}{$\begin{array}{c}\text { Courtyard type -1 (1:1.5) } \\
\text { November -February energy }\end{array}$}} & & \multicolumn{2}{|l|}{ North } & \multicolumn{2}{|l|}{ East } & \multicolumn{2}{|l|}{ South } & \multicolumn{2}{|l|}{ West } \\
\hline & & & Daylight & Above threshold \% & Decrease \% & Above threshold \% & Decrease \% & Above threshold \% & Decrease \% & Above threshold \% & Decrease \% \\
\hline \multirow{8}{*}{ Glazing } & \multirow{8}{*}{$\begin{array}{l}\text { Courtyard Trp Clr } \\
3 \mathrm{~mm} / 13 \mathrm{~mm} \text { Air } \\
\text { and sky light Dbl } \\
\text { Clr } 6 \mathrm{~mm} / 13 \mathrm{~mm} \text { Air }\end{array}$} & \multirow{2}{*}{$77 \%$} & Court & 99.898 & -0.8 & 99.796 & -0.5 & 99.796 & -0.9 & 99.898 & -0.9 \\
\hline & & & Atrium & 99.081 & & 99.285 & & 98.876 & & 98.978 & \\
\hline & & \multirow{2}{*}{$57 \%$} & Court & 99.694 & -1.7 & 99.591 & -0.9 & 99.898 & -2.4 & 99.796 & -1.9 \\
\hline & & & Atrium & 97.957 & & 98.672 & & 97.548 & & 97.855 & \\
\hline & & \multirow{2}{*}{$36 \%$} & Court & 83.962 & -17.8 & 83.451 & -16.0 & 84.984 & -17.7 & 83.86 & -17.7 \\
\hline & & & Atrium & 69.027 & & 70.137 & & 69.935 & & 69.027 & \\
\hline & & \multirow{2}{*}{$20 \%$} & Court & 61.864 & -6.9 & 62.873 & -8.0 & 61.46 & -6.4 & 62.065 & -6.3 \\
\hline & & & Atrium & 57.626 & & 57.828 & & 57.525 & & 58.131 & \\
\hline
\end{tabular}

Appendix XXII Daylighting condition of configuration -1, Option - 6

\begin{tabular}{|c|c|c|c|c|c|c|c|c|c|c|c|}
\hline \multirow{2}{*}{\multicolumn{3}{|c|}{$\begin{array}{c}\text { Courtyard type -1 (1:1.5) } \\
\text { November -February energy }\end{array}$}} & & \multicolumn{2}{|l|}{ North } & \multicolumn{2}{|l|}{ East } & \multicolumn{2}{|l|}{ South } & \multicolumn{2}{|l|}{ West } \\
\hline & & & Daylight & Above threshold \% & Decrease \% & Above threshold \% & Decrease \% & Above threshold \% & Decrease \% & Above threshold \% & Decrease \% \\
\hline \multirow{8}{*}{ Glazing } & \multirow{8}{*}{$\begin{array}{c}\text { Courtyard Trp Clr } \\
3 \mathrm{~mm} / 13 \mathrm{~mm} \text { Air } \\
\text { and sky light Dbl } \\
\text { LoE }(e 2=.1) \mathrm{Clr} \\
6 \mathrm{~mm} / 13 \mathrm{~mm} \text { Air }\end{array}$} & \multirow{2}{*}{$77 \%$} & Court & 99.898 & -1.8 & 99.796 & -1.7 & 99.898 & -1.4 & 99.796 & -1.0 \\
\hline & & & Atrium & 98.059 & & 98.059 & & 98.468 & & 98.774 & \\
\hline & & \multirow{2}{*}{$57 \%$} & Court & 99.694 & -2.9 & 99.796 & -2.6 & 99.694 & -2.6 & 99.694 & -2.2 \\
\hline & & & Atrium & 96.833 & & 97.242 & & 97.14 & & 97.548 & \\
\hline & & \multirow{2}{*}{$36 \%$} & Court & 84.269 & -7.9 & 84.779 & -8.8 & 83.962 & -6.0 & 83.962 & -6.8 \\
\hline & & & Atrium & 77.629 & & 77.322 & & 78.957 & & 78.242 & \\
\hline & & \multirow{2}{*}{$20 \%$} & Court & 70.172 & -5.8 & 70.682 & -22.3 & 70.785 & -23.1 & 70.785 & -22.9 \\
\hline & & & Atrium & 66.086 & & 54.951 & & 54.44 & & 54.542 & \\
\hline
\end{tabular}

Appendix XXIII Daylighting condition of configuration -1, Option - 7

\begin{tabular}{|c|c|c|c|c|c|c|c|c|c|c|c|}
\hline \multirow{2}{*}{\multicolumn{3}{|c|}{$\begin{array}{l}\text { Courtyard type -1 (1:1.5) } \\
\text { November -February energy }\end{array}$}} & & \multicolumn{2}{|l|}{ North } & \multicolumn{2}{|l|}{ East } & \multicolumn{2}{|l|}{ South } & \multicolumn{2}{|l|}{ West } \\
\hline & & & \multirow{2}{*}{$\begin{array}{c}\text { Daylight } \\
\text { Court }\end{array}$} & \multirow{2}{*}{\begin{tabular}{|c|} 
Above threshold \% \\
99.694 \\
\end{tabular}} & \multirow{2}{*}{\begin{tabular}{|c|} 
Decrease \% \\
-1.5 \\
\end{tabular}} & \multirow{2}{*}{\begin{tabular}{|c|} 
Above threshold \% \\
99.796
\end{tabular}} & \multirow{2}{*}{\begin{tabular}{|c|} 
Decrease \% \\
-1.4
\end{tabular}} & \multirow{2}{*}{\begin{tabular}{|c|} 
Above threshold \% \\
99.898 \\
\end{tabular}} & \multirow{2}{*}{\begin{tabular}{|c|} 
Decrease \% \\
-3.1
\end{tabular}} & \multirow{2}{*}{\begin{tabular}{|c|} 
Above threshold \% \\
99.796 \\
\end{tabular}} & \multirow{2}{*}{\begin{tabular}{|c|} 
Decrease \% \\
-2.5
\end{tabular}} \\
\hline \multirow{8}{*}{ Glazing } & \multirow{8}{*}{$\begin{array}{c}\text { Courtyard Trp LoE } \\
(\mathrm{e} 2=\mathrm{e} 5=.1) \mathrm{Clr} \\
3 \mathrm{~mm} / 13 \mathrm{~mm} \text { Air } \\
\text { and sky light Dbl } \\
\text { Clr } 6 \mathrm{~mm} / 13 \mathrm{~mm} \text { Air }\end{array}$} & \multirow{2}{*}{$77 \%$} & & & & & & & & & \\
\hline & & & Atrium & 98.161 & & 98.366 & & 96.833 & & 97.344 & \\
\hline & & \multirow{2}{*}{$57 \%$} & Court & 99.489 & -3.5 & 99.591 & -3.3 & 99.489 & -4.0 & 99.694 & -4.0 \\
\hline & & & Atrium & 96.016 & & 96.323 & & 95.505 & & 95.71 & \\
\hline & & \multirow{2}{*}{$36 \%$} & Court & 80.08 & -6.6 & 79.263 & -5.0 & 81 & -7.2 & 80.08 & -6.1 \\
\hline & & & Atrium & 74.768 & & 75.279 & & 75.177 & & 75.177 & \\
\hline & & \multirow{2}{*}{$20 \%$} & Court & 67.311 & -5.5 & 67.107 & -5.9 & 68.537 & -7.8 & 67.72 & -8.9 \\
\hline & & & Atrium & 63.634 & & 63.123 & & 63.225 & & 61.693 & \\
\hline
\end{tabular}

Appendix XXIV Daylighting condition of configuration -1, Option - 8

\begin{tabular}{|c|c|c|c|c|c|c|c|c|c|c|c|}
\hline \multicolumn{3}{|c|}{ Courtyard type -1 (1:1.5) } & & \multicolumn{2}{|l|}{ North } & \multicolumn{2}{|l|}{ East } & \multicolumn{2}{|l|}{ South } & \multicolumn{2}{|l|}{ West } \\
\hline & ember -February en & & Daylight & Above threshold \% & Decrease \% & Above threshold \% & Decrease \% & Above threshold \% & Decrease \% & Above threshold \% & Decrease \% \\
\hline \multirow{8}{*}{ Glazing } & \multirow{8}{*}{$\begin{array}{l}\text { Courtyard Trp LoE } \\
\text { (e2=e5=.1) Clr } \\
3 \mathrm{~mm} / 13 \mathrm{~mm} \text { Air } \\
\text { and sky light Dbl } \\
\text { LoE (e2=.1) Clr } \\
6 \mathrm{~mm} / 13 \mathrm{~mm} \text { Air }\end{array}$} & \multirow{2}{*}{$77 \%$} & Court & 99.694 & -3.0 & 99.694 & -2.9 & 99.796 & -2.8 & 99.694 & -3.1 \\
\hline & & & Atrium & 96.731 & & 96.833 & & 97.038 & & 96.629 & \\
\hline & & \multirow{2}{*}{$57 \%$} & Court & 99.591 & -3.8 & 99.694 & -3.8 & 99.489 & -4.0 & 99.591 & -2.9 \\
\hline & & & Atrium & 95.812 & & 95.914 & & 95.505 & & 96.731 & \\
\hline & & \multirow{2}{*}{$36 \%$} & Court & 79.978 & -7.7 & 80.285 & -7.5 & 81.204 & -8.2 & 81 & -7.9 \\
\hline & & & Atrium & 73.849 & & 74.258 & & 74.564 & & 74.564 & \\
\hline & & \multirow{2}{*}{$20 \%$} & Court & 67.107 & -7.2 & 67.822 & -8.3 & 66.596 & -6.3 & 66.801 & -8.4 \\
\hline & & & Atrium & 62.306 & & 62.204 & & 62.408 & & 61.182 & \\
\hline
\end{tabular}

Appendix XXV Daylighting condition of configuration -2, Option - 1

\begin{tabular}{|c|c|c|c|c|c|c|c|c|c|c|c|}
\hline \multicolumn{4}{|c|}{\begin{tabular}{|c|} 
Courtyard type -2 (1:1.) \\
\end{tabular}} & \multicolumn{2}{|l|}{ North } & \multicolumn{2}{|l|}{ East } & \multicolumn{2}{|l|}{ South } & \multicolumn{2}{|l|}{ West } \\
\hline \multicolumn{3}{|c|}{ November - April energy } & Daylight & $\%$ of area above threshold & Decrease \% & $\%$ of area above threshold & Decrease \% & $\%$ of area above threshold & Decrease \% & $\%$ of area above threshold & Decrease \% \\
\hline \multirow{8}{*}{ Glazing } & \multirow{8}{*}{$\begin{array}{c}\text { Courtyard Dble clr, } \\
6 \mathrm{~mm} / 13 \mathrm{~mm} \text { air } \\
\text { and sky light Dble } \\
\mathrm{clr}, 6 \mathrm{~mm} / 13 \mathrm{~mm} \text { air }\end{array}$} & \multirow{2}{*}{$73 \%$} & Court & 99.395 & -7.2 & 99.294 & -6.3 & 99.294 & -8.5 & 99.395 & -7.7 \\
\hline & & & Atrium & 92.231 & & 93.039 & & 90.819 & & 91.727 & \\
\hline & & \multirow{2}{*}{$55 \%$} & Court & 99.092 & -7.5 & 97.982 & -7.3 & 98.487 & -7.3 & 98.487 & -9.0 \\
\hline & & & Atrium & 91.626 & & 90.819 & & 91.323 & & 89.608 & \\
\hline & & \multirow{2}{*}{$36 \%$} & Court & 77.098 & -7.9 & 79.419 & -10.4 & 77.502 & -8.9 & 78.006 & -10.9 \\
\hline & & & Atrium & 71.045 & & 71.146 & & 70.641 & & 69.531 & \\
\hline & & \multirow{2}{*}{$23 \%$} & Court & 64.386 & -6.9 & 63.074 & -6.1 & 64.588 & -6.7 & 63.276 & -8.1 \\
\hline & & & Atrium & 59.947 & & 59.241 & & 60.249 & & 58.131 & \\
\hline
\end{tabular}


Appendix XXVI Daylighting condition of configuration -2, Option - 2

\begin{tabular}{|c|c|c|c|c|c|c|c|c|c|c|c|}
\hline \multicolumn{4}{|c|}{\begin{tabular}{|l|} 
Courtyard type -2 (1:1) \\
\end{tabular}} & \multicolumn{2}{|l|}{ North } & \multicolumn{2}{|l|}{ East } & \multicolumn{2}{|l|}{ South } & \multicolumn{2}{|l|}{ West } \\
\hline Novembe & $r$-April energy & & Daylight & $\%$ of area above threshold & Decrease \% & $\%$ of area above threshold & Decrease \% & $\%$ of area above threshold & Decrease \% & $\%$ of area above threshold & Decrease \% \\
\hline \multirow{8}{*}{ Glazing } & \multirow{8}{*}{$\begin{array}{c}\text { Courtyard Dble clr, } \\
6 \mathrm{~mm} / 13 \mathrm{~mm} \text { air } \\
\text { and sky light Dbl } \\
\text { LoE (e2=.1) Clr } \\
6 \mathrm{~mm} / 13 \mathrm{~mm} \mathrm{Air}\end{array}$} & \multirow{2}{*}{$73 \%$} & Court & 99.395 & \begin{tabular}{|l|}
-7.3 \\
\end{tabular} & 99.193 & \begin{tabular}{|l|}
-7.7 \\
\end{tabular} & 99.496 & -9.0 & 98.991 & \begin{tabular}{|l|}
-9.7 \\
\end{tabular} \\
\hline & & & Atrium & 92.131 & & 91.525 & & 90.516 & & 89.407 & \\
\hline & & \multirow{2}{*}{$55 \%$} & Court & 98.487 & -10.0 & 97.881 & -8.9 & 98.588 & -9.2 & 97.78 & -9.1 \\
\hline & & & Atrium & 88.599 & & 89.205 & & 89.507 & & 88.902 & \\
\hline & & \multirow{2}{*}{$36 \%$} & Court & 79.015 & -10.7 & 79.519 & -12.2 & 78.309 & -10.3 & 77.401 & -8.7 \\
\hline & & & Atrium & 70.54 & & 69.834 & & 70.238 & & 70.641 & \\
\hline & & \multirow{2}{*}{$23 \%$} & Court & 64.083 & -9.3 & 65.193 & -9.0 & 64.89 & -9.3 & 64.789 & -9.0 \\
\hline & & & Atrium & 58.131 & & 59.341 & & 58.837 & & 58.938 & \\
\hline
\end{tabular}

Appendix XXVII Daylighting condition of configuration -2, Option - 3

\begin{tabular}{|c|c|c|c|c|c|c|c|c|c|c|c|}
\hline \multicolumn{4}{|c|}{ Courtyard type -2 (1:1) } & \multicolumn{2}{|l|}{ North } & \multicolumn{2}{|l|}{ East } & \multicolumn{2}{|l|}{ South } & \multicolumn{2}{|l|}{ West } \\
\hline \multicolumn{3}{|c|}{ November - April energy } & Daylight & Above threshold \% & Decrease \% & Above threshold \% & Decrease \% & Above threshold \% & Decrease \% & Above threshold \% & Decrease \% \\
\hline \multirow{8}{*}{ Glazing } & \multirow{8}{*}{$\begin{array}{c}\text { Courtyard Dbl LoE } \\
\text { (e2=.1) Clr } \\
6 \mathrm{~mm} / 13 \mathrm{~mm} \text { Air } \\
\text { and sky light Dble } \\
\text { clr, } 6 \mathrm{~mm} / 13 \mathrm{~mm} \text { air }\end{array}$} & \multirow{2}{*}{$73 \%$} & Court & 98.789 & -8.8 & 98.588 & -7.4 & 99.092 & -9.0 & 98.89 & -10.4 \\
\hline & & & Atrium & 90.113 & & 91.323 & & 90.214 & & 88.599 & \\
\hline & & \multirow{2}{*}{$55 \%$} & Court & 97.074 & -8.6 & 97.579 & -9.3 & 97.276 & -11.4 & 97.881 & -7.3 \\
\hline & & & Atrium & 88.7 & & 88.499 & & 86.178 & & 90.718 & \\
\hline & & \multirow{2}{*}{$36 \%$} & Court & 77.3 & -10.7 & 77.3 & -8.7 & 75.786 & -8.5 & 77.098 & -9.2 \\
\hline & & & Atrium & 69.027 & & 70.54 & & 69.33 & & 70.036 & \\
\hline & & \multirow{2}{*}{$23 \%$} & Court & 62.973 & -6.9 & 63.478 & -8.6 & 63.175 & -8.6 & 64.083 & -9.1 \\
\hline & & & Atrium & 58.635 & & 58.03 & & 57.727 & & 58.232 & \\
\hline
\end{tabular}

Appendix XXVIII Daylighting condition of configuration -2, Option - 4

\begin{tabular}{|c|c|c|c|c|c|c|c|c|c|c|c|}
\hline \multicolumn{4}{|c|}{ Courtyard type -2 (1:1) } & \multicolumn{2}{|l|}{ North } & \multicolumn{2}{|l|}{ East } & \multicolumn{2}{|l|}{ South } & \multicolumn{2}{|l|}{ West } \\
\hline \multicolumn{3}{|c|}{ November - April energy } & Daylight & Above threshold \% & Decrease \% & Above threshold \% & Decrease \% & Above threshold \% & Decrease \% & Above threshold \% & Decrease \% \\
\hline \multirow{8}{*}{ Glazing } & \multirow{8}{*}{$\begin{array}{c}\text { Courtyard Dbl LoE } \\
(\mathrm{e} 2=.1) \mathrm{Clr} \\
6 \mathrm{~mm} / 13 \mathrm{~mm} \text { Air } \\
\text { and sky light Dbl } \\
\text { LoE (e2=.1) Clr } \\
6 \mathrm{~mm} / 13 \mathrm{~mm} \text { Air }\end{array}$} & \multirow{2}{*}{$73 \%$} & Court & 99.395 & \begin{tabular}{|l|}
-9.8 \\
\end{tabular} & 98.991 & -9.4 & 99.294 & -9.3 & 98.588 & -10.4 \\
\hline & & & Atrium & 89.608 & & 89.709 & & 90.012 & & 88.297 & \\
\hline & & \multirow{2}{*}{$55 \%$} & Court & 97.68 & -11.4 & 97.982 & -10.8 & 97.579 & -11.8 & 96.872 & -9.8 \\
\hline & & & Atrium & 86.582 & & 87.389 & & 86.077 & & 87.389 & \\
\hline & & \multirow{2}{*}{$36 \%$} & Court & 75.383 & -8.0 & 77.098 & -10.1 & 75.887 & -8.1 & 76.795 & -10.0 \\
\hline & & & Atrium & 69.33 & & 69.33 & & 69.733 & & 69.128 & \\
\hline & & \multirow{2}{*}{$23 \%$} & Court & 62.166 & -7.6 & 62.671 & -8.2 & 64.588 & -9.7 & 61.965 & -6.8 \\
\hline & & & Atrium & 57.425 & & 57.525 & & 58.333 & & 57.727 & \\
\hline
\end{tabular}

Appendix XXIX Daylighting condition of configuration -2, Option - 5

\begin{tabular}{|c|c|c|c|c|c|c|c|c|c|c|c|}
\hline \multicolumn{4}{|c|}{ Courtyard type -2 (1:1) } & \multicolumn{2}{|l|}{ North } & \multicolumn{2}{|l|}{ East } & \multicolumn{2}{|l|}{ South } & \multicolumn{2}{|l|}{ West } \\
\hline Novembe & $r$-April energy & & Daylight & Above threshold \% & Decrease \% & Above threshold \% & Decrease \% & Above threshold \% & Decrease \% & Above threshold \% & Decrease \% \\
\hline \multirow{8}{*}{ Glazing } & \multirow{8}{*}{$\begin{array}{l}\text { Courtyard Trp Clr } \\
3 \mathrm{~mm} / 13 \mathrm{~mm} \text { Air } \\
\text { and sky light Dbl } \\
\text { Clr } 6 \mathrm{~mm} / 13 \mathrm{~mm} \text { Air }\end{array}$} & \multirow{2}{*}{$73 \%$} & Court & 98.588 & -9.4 & 99.193 & -10.9 & 98.991 & -9.0 & 99.193 & -9.1 \\
\hline & & & Atrium & 89.306 & & 88.398 & & 90.113 & & 90.214 & \\
\hline & & \multirow{2}{*}{$55 \%$} & Court & 97.68 & -10.8 & 97.68 & -10.0 & 97.579 & -11.9 & 97.478 & -9.1 \\
\hline & & & Atrium & 87.086 & & 87.893 & & 85.976 & & 88.599 & \\
\hline & & \multirow{2}{*}{$36 \%$} & Court & 76.795 & -10.9 & 75.484 & -6.9 & 75.988 & -8.6 & 75.686 & -9.5 \\
\hline & & & Atrium & 68.422 & & 70.238 & & 69.43 & & 68.522 & \\
\hline & & \multirow{2}{*}{$23 \%$} & Court & 62.469 & -8.9 & 62.267 & -8.4 & 62.267 & -7.3 & 61.864 & -8.2 \\
\hline & & & Atrium & 56.92 & & 57.021 & & 57.727 & & 56.819 & \\
\hline
\end{tabular}

Appendix XXX Daylighting condition of configuration -2, Option - 6

\begin{tabular}{|c|c|c|c|c|c|c|c|c|c|c|c|}
\hline \multicolumn{4}{|c|}{ Courtyard type -2 (1:1) } & \multicolumn{2}{|l|}{ North } & \multicolumn{2}{|l|}{ East } & \multicolumn{2}{|l|}{ South } & \multicolumn{2}{|l|}{ West } \\
\hline \multicolumn{3}{|c|}{ November - April energy } & Daylight & Above threshold \% & Decrease \% & Above threshold \% & Decrease \% & Above threshold \% & Decrease \% & Above threshold \% & Decrease \% \\
\hline \multirow{8}{*}{ Glazing } & \multirow{8}{*}{$\begin{array}{l}\text { Courtyard Trp Clr } \\
\text { 3mm/13mm Air } \\
\text { and sky light Dbl } \\
\text { LoE (e2=.1) Clr } \\
6 \mathrm{~mm} / 13 \mathrm{~mm} \text { Air }\end{array}$} & \multirow{2}{*}{$73 \%$} & Court & 98.789 & \begin{tabular}{|l|}
-11.6 \\
\end{tabular} & 98.487 & \begin{tabular}{|l|}
-7.5 \\
\end{tabular} & 97.579 & \begin{tabular}{|l|}
-7.3 \\
\end{tabular} & 98.89 & -11.2 \\
\hline & & & Atrium & 87.288 & & 91.122 & & 90.415 & & 87.792 & \\
\hline & & \multirow{2}{*}{$55 \%$} & Court & 98.386 & -12.8 & 97.982 & -11.9 & 96.973 & -11.4 & 98.083 & -12.1 \\
\hline & & & Atrium & 85.775 & & 86.279 & & 85.875 & & 86.178 & \\
\hline & & \multirow{2}{*}{$36 \%$} & Court & 75.988 & -9.4 & 76.896 & -10.0 & 75.786 & -8.0 & 76.594 & -10.1 \\
\hline & & & Atrium & 68.825 & & 69.229 & & 69.733 & & 68.825 & \\
\hline & & \multirow{2}{*}{$23 \%$} & Court & 62.469 & -9.0 & 62.166 & -7.8 & 62.469 & -7.9 & 62.469 & -8.1 \\
\hline & & & Atrium & 56.819 & & 57.324 & & 57.525 & & 57.425 & \\
\hline
\end{tabular}


Appendix XXXI Daylighting condition of configuration -2, Option - 7

\begin{tabular}{|c|c|c|c|c|c|c|c|c|c|c|c|}
\hline \multicolumn{4}{|c|}{ Courtyard type -2 (1:1) } & \multicolumn{2}{|l|}{ North } & \multicolumn{2}{|l|}{ East } & \multicolumn{2}{|l|}{ South } & \multicolumn{2}{|l|}{ West } \\
\hline \multicolumn{3}{|c|}{ November - April energy } & Daylight & Above threshold \% & Decrease \% & Above threshold \% & Decrease \% & Above threshold \% & Decrease \% & Above threshold $\%$ & Decrease \% \\
\hline \multirow{8}{*}{ Glazing } & \multirow{8}{*}{$\begin{array}{c}\text { Courtyard Trp LoE } \\
\text { (e2=e5=.1) Clr } \\
3 \mathrm{~mm} / 13 \mathrm{~mm} \text { Air } \\
\text { and sky light Dbl } \\
\text { Clr } 6 \mathrm{~mm} / 13 \mathrm{~mm} \text { Air }\end{array}$} & \multirow{2}{*}{$73 \%$} & Court & 97.579 & -12.5 & 97.175 & -12.3 & 97.276 & \begin{tabular}{|l|}
-13.1 \\
\end{tabular} & 97.478 & -11.5 \\
\hline & & & Atrium & 85.371 & & 85.27 & & 84.564 & & 86.279 & \\
\hline & & \multirow{2}{*}{$55 \%$} & Court & 95.157 & -12.1 & 94.451 & -12.5 & 95.46 & -12.8 & 94.956 & -12.4 \\
\hline & & & Atrium & 83.656 & & 82.647 & & 83.252 & & 83.151 & \\
\hline & & \multirow{2}{*}{$36 \%$} & Court & 72.054 & -11.1 & 72.659 & -10.1 & 72.154 & -9.4 & 71.549 & -10.4 \\
\hline & & & Atrium & 64.083 & & 65.294 & & 65.395 & & 64.083 & \\
\hline & & \multirow{2}{*}{$23 \%$} & Court & 57.223 & -5.6 & 59.846 & -9.4 & 58.131 & -5.9 & 59.442 & -8.3 \\
\hline & & & Atrium & 53.994 & & 54.196 & & 54.701 & & 54.499 & \\
\hline
\end{tabular}

Appendix XXXII Daylighting condition of configuration -2, Option - 8

\begin{tabular}{|c|c|c|c|c|c|c|c|c|c|c|c|}
\hline \multicolumn{4}{|c|}{ Courtyard type -2 (1:1) } & \multicolumn{2}{|l|}{ North } & \multicolumn{2}{|l|}{ East } & \multicolumn{2}{|l|}{ South } & \multicolumn{2}{|l|}{ West } \\
\hline \multicolumn{3}{|c|}{ November - April energy } & \begin{tabular}{|l|} 
Daylight \\
\end{tabular} & Above threshold \% & Decrease \% & Above threshold \% & Decrease \% & Above threshold \% & Decrease \% & Above threshold \% & Decrease \% \\
\hline \multirow{8}{*}{ Glazing } & \multirow{8}{*}{$\begin{array}{l}\text { Courtyard Trp LoE } \\
(\mathrm{e} 2=\mathrm{e} 5=.1) \mathrm{Clr} \\
3 \mathrm{~mm} / 13 \mathrm{~mm} \text { Air } \\
\text { and sky light Dbl } \\
\text { LoE (e2=.1) Clr } \\
6 \mathrm{~mm} / 13 \mathrm{~mm} \text { Air }\end{array}$} & \multirow{2}{*}{$73 \%$} & Court & 97.276 & \begin{tabular}{|l|}
-13.0 \\
\end{tabular} & 96.469 & \begin{tabular}{|l|}
-11.6 \\
\end{tabular} & 95.864 & -13.5 & 96.469 & -13.2 \\
\hline & & & Atrium & 84.665 & & 85.27 & & 82.95 & & 83.757 & \\
\hline & & \multirow{2}{*}{$55 \%$} & Court & 95.864 & -14.7 & 96.872 & -17.3 & 94.451 & -13.4 & 95.157 & -14.0 \\
\hline & & & Atrium & 81.739 & & 80.125 & & 81.84 & & 81.84 & \\
\hline & & \multirow{2}{*}{$36 \%$} & Court & 71.549 & -9.4 & 72.154 & -10.1 & 71.751 & -9.8 & 72.861 & -13.3 \\
\hline & & & Atrium & 64.789 & & 64.89 & & 64.689 & & 63.175 & \\
\hline & & \multirow{2}{*}{$23 \%$} & Court & 57.727 & -7.7 & 59.442 & -9.0 & 59.241 & -9.2 & 57.929 & -7.0 \\
\hline & & & Atrium & 53.288 & & 54.095 & & 53.793 & & 53.893 & \\
\hline
\end{tabular}

Appendix XXXIII Improvement in energy consumption by atrium house compare to courtyard house

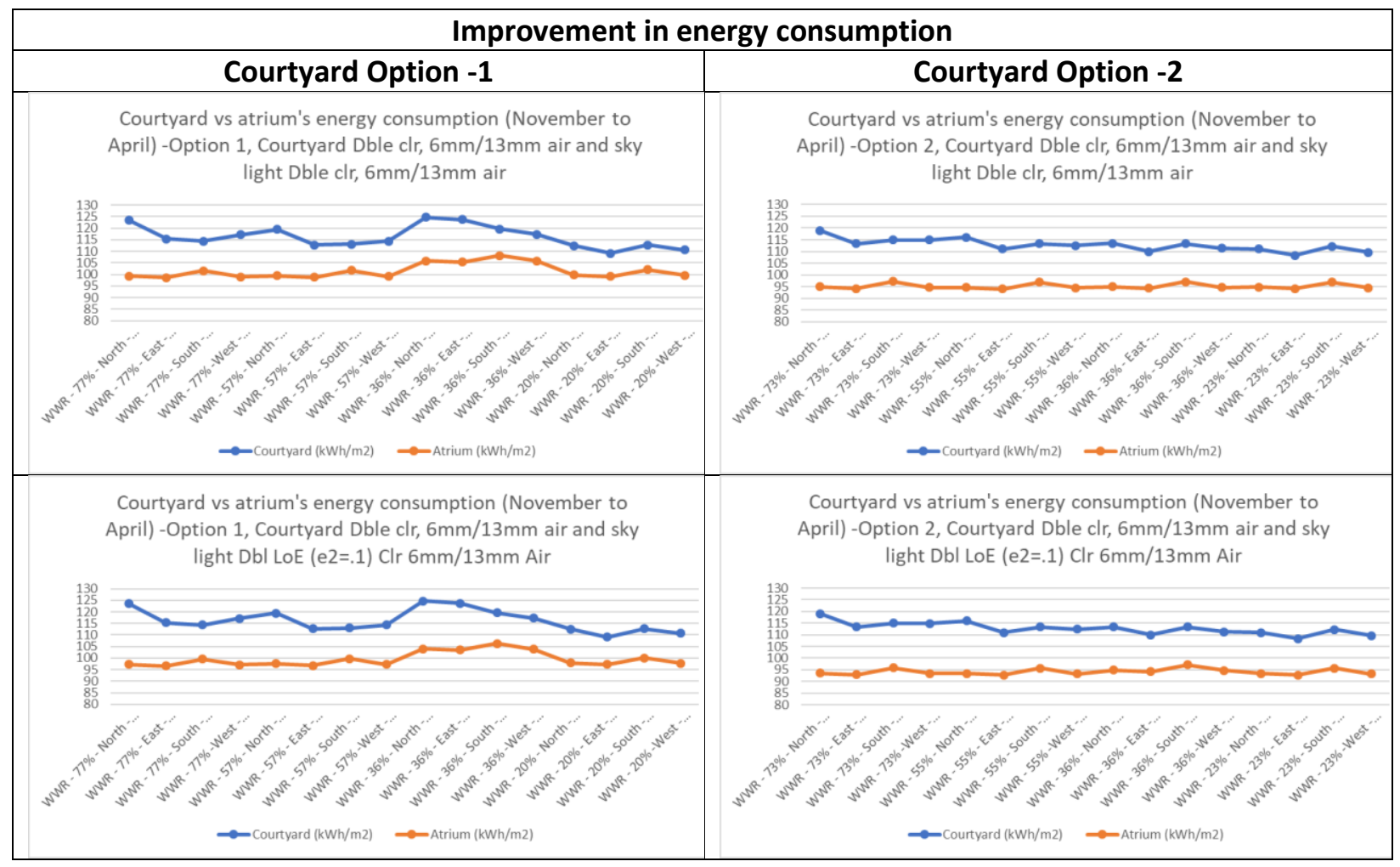




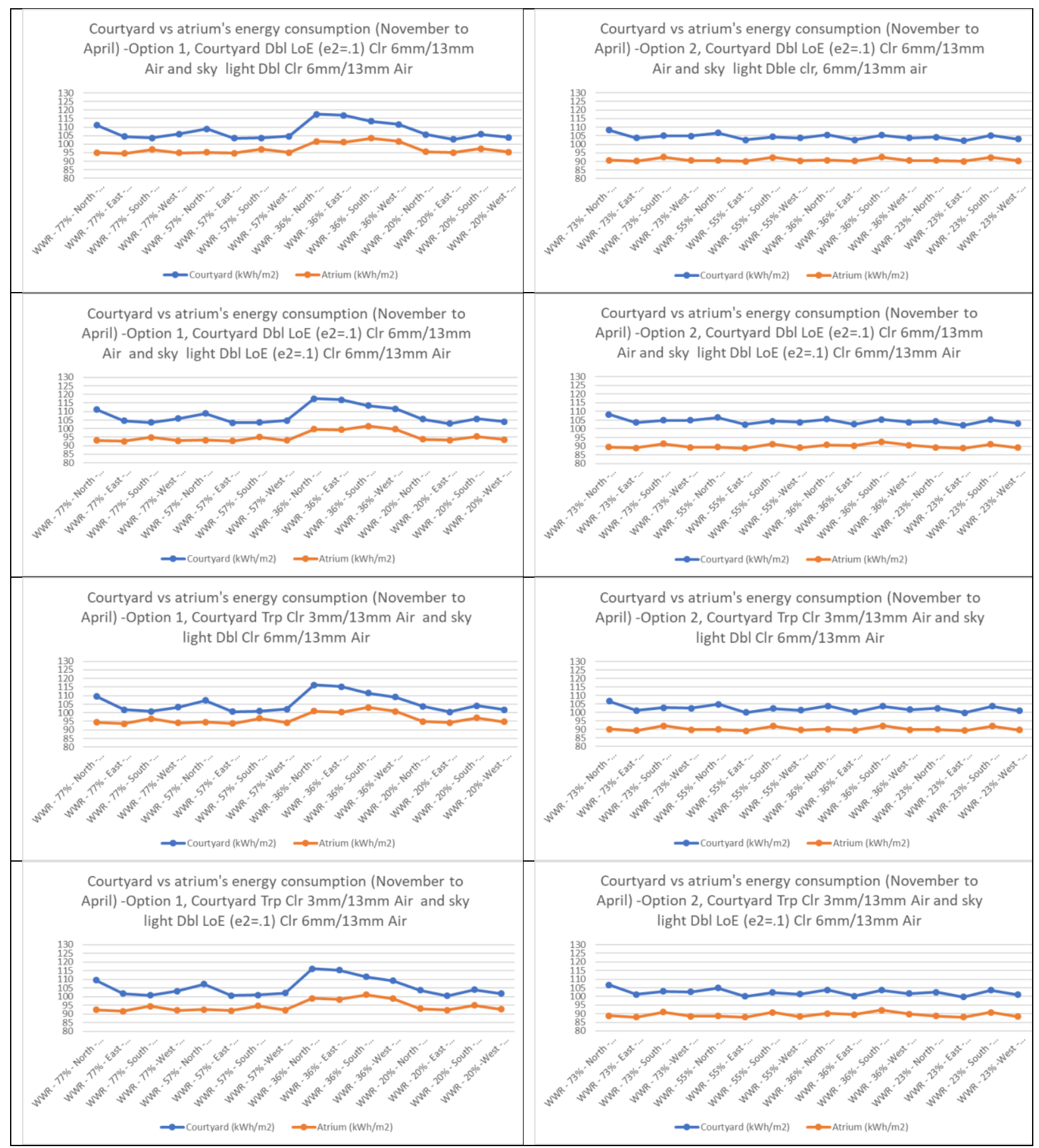




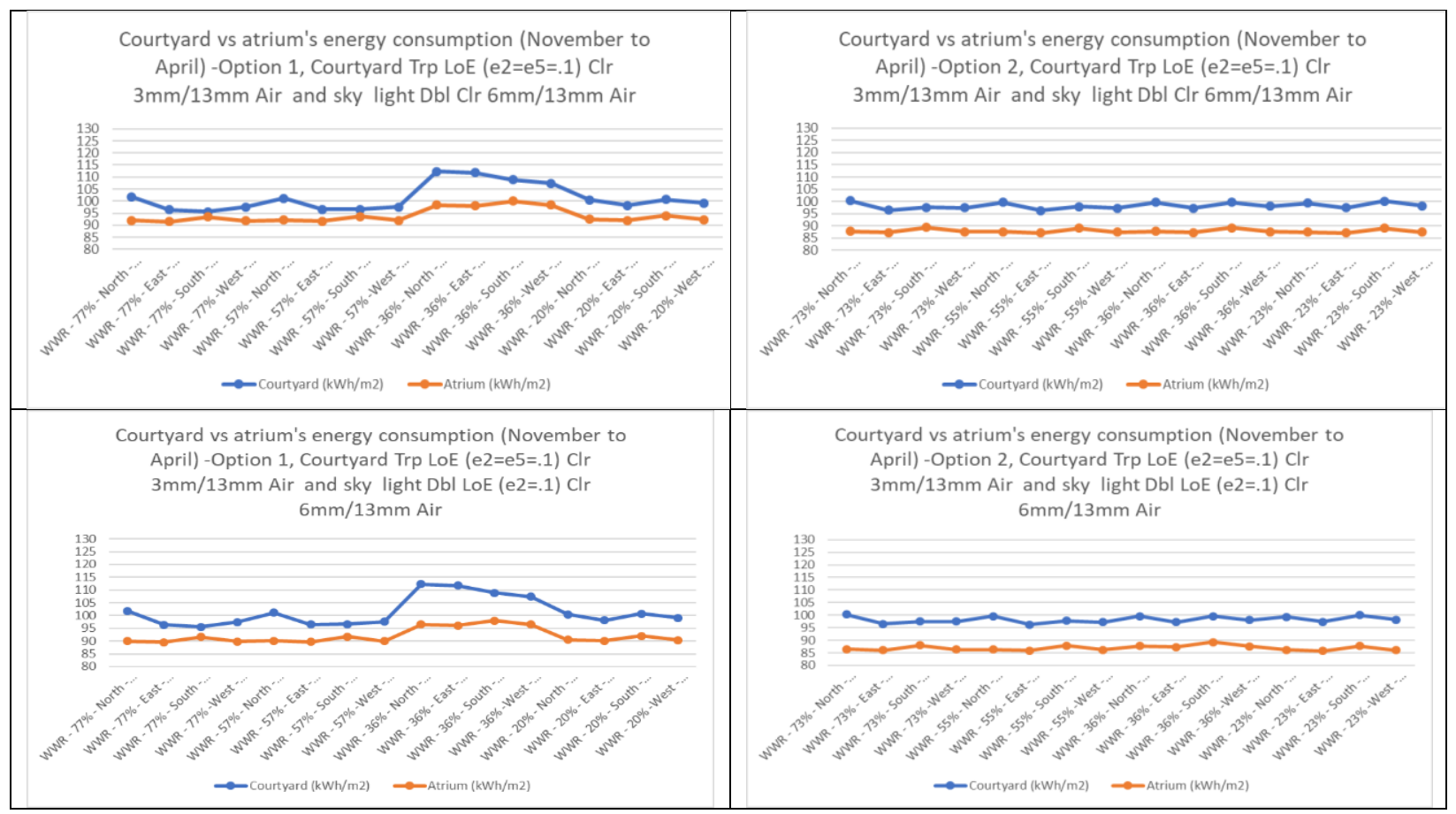

Appendix XXXIV Energy consumption of atrium house with different WWR

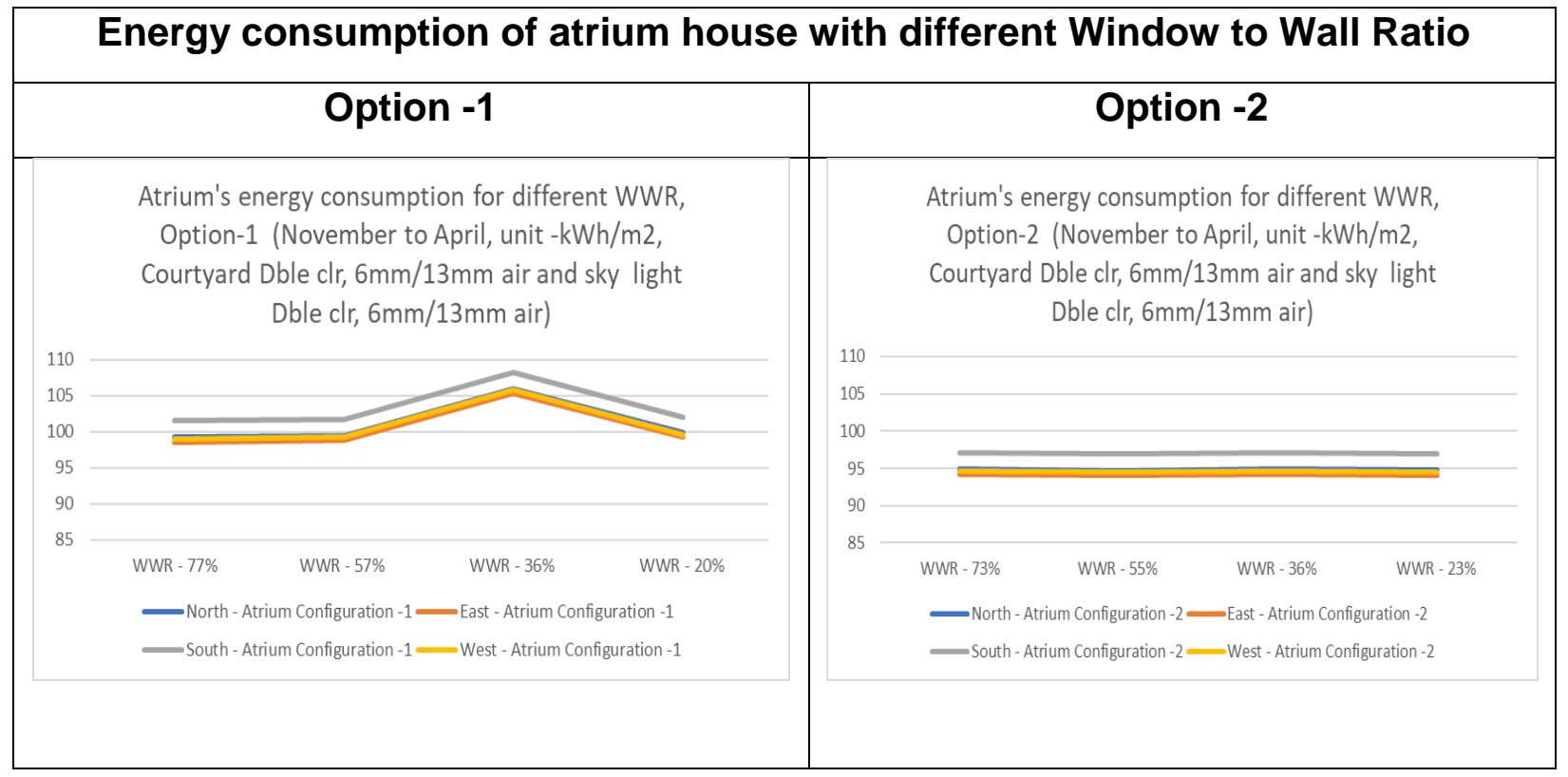




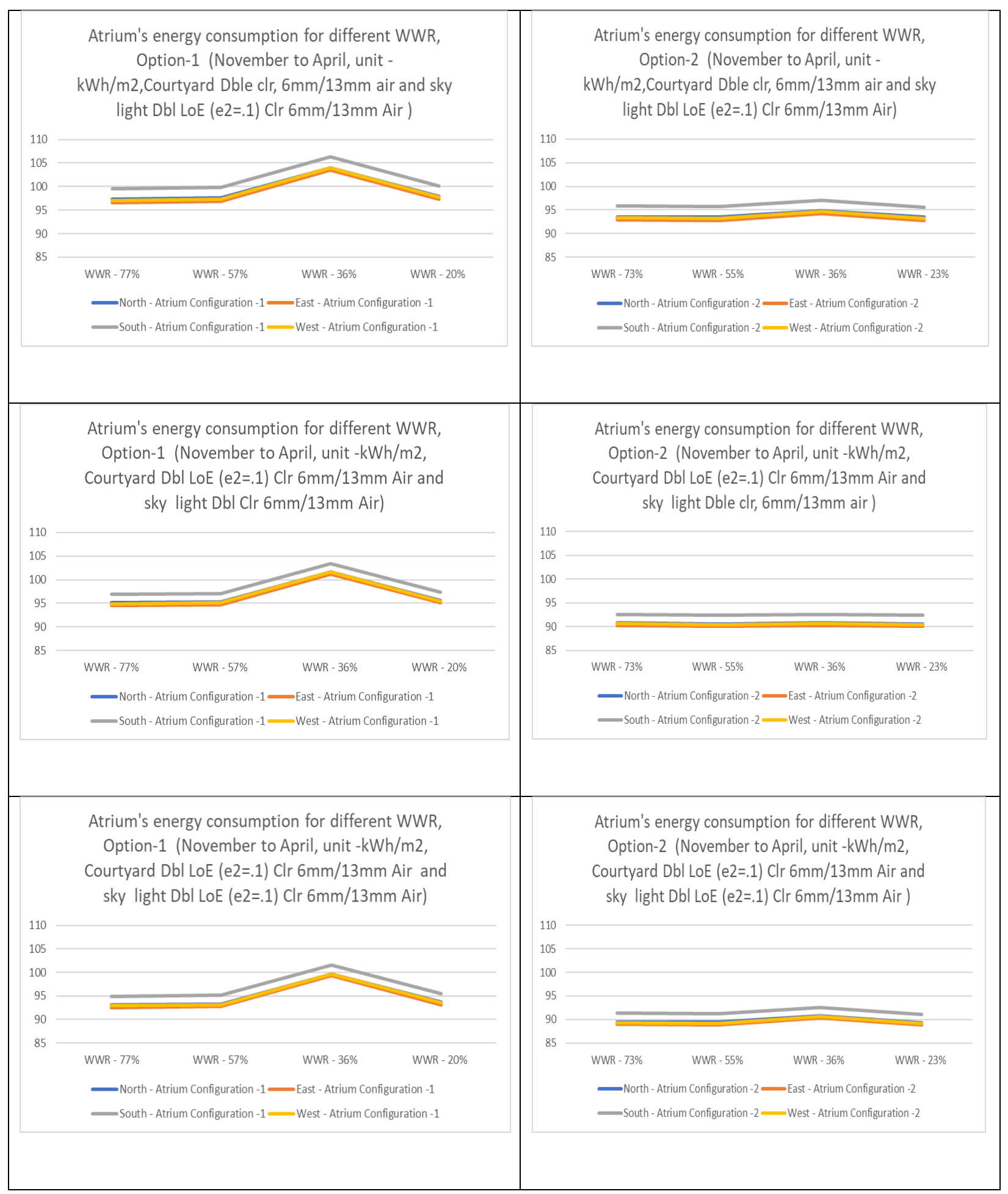




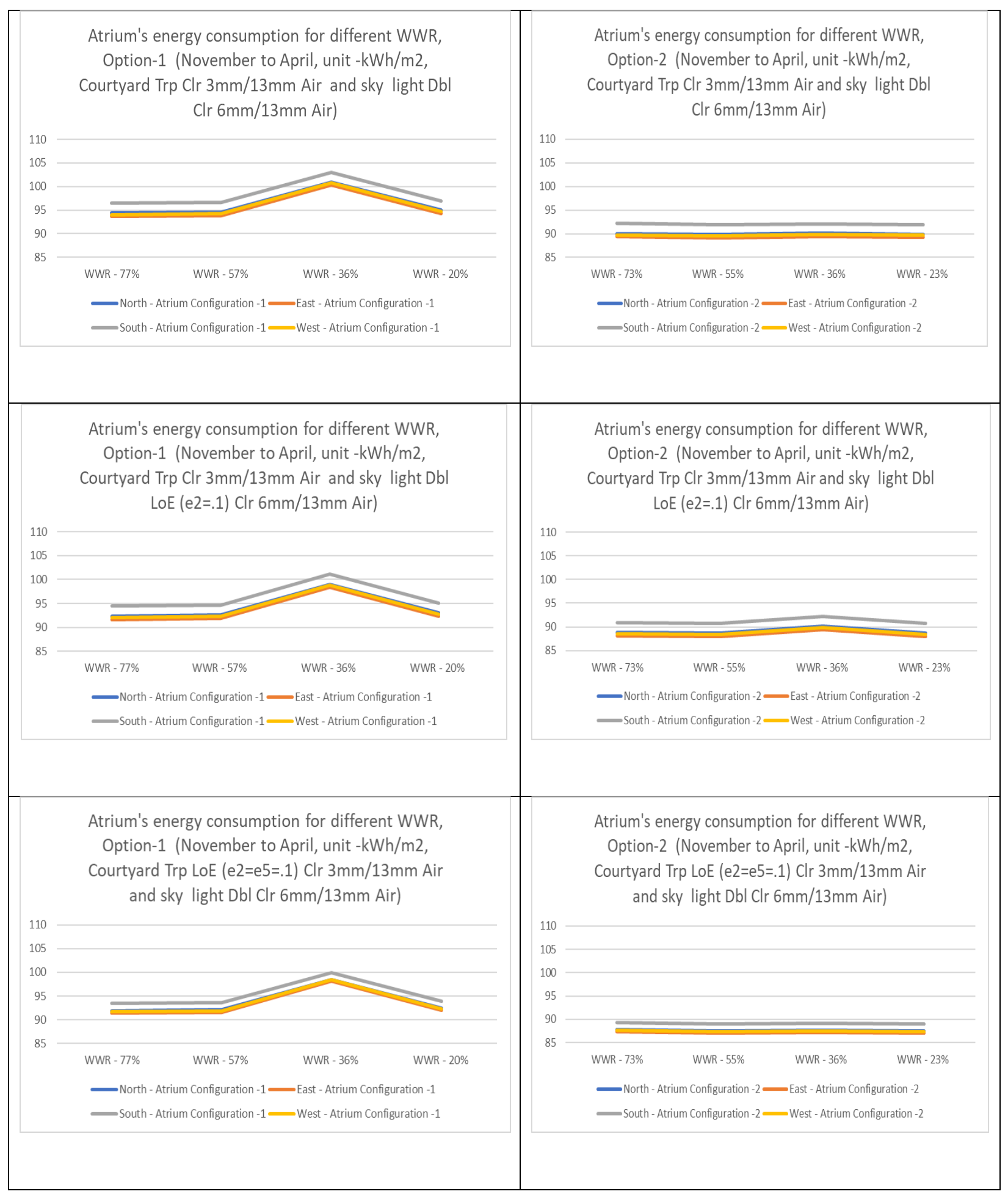




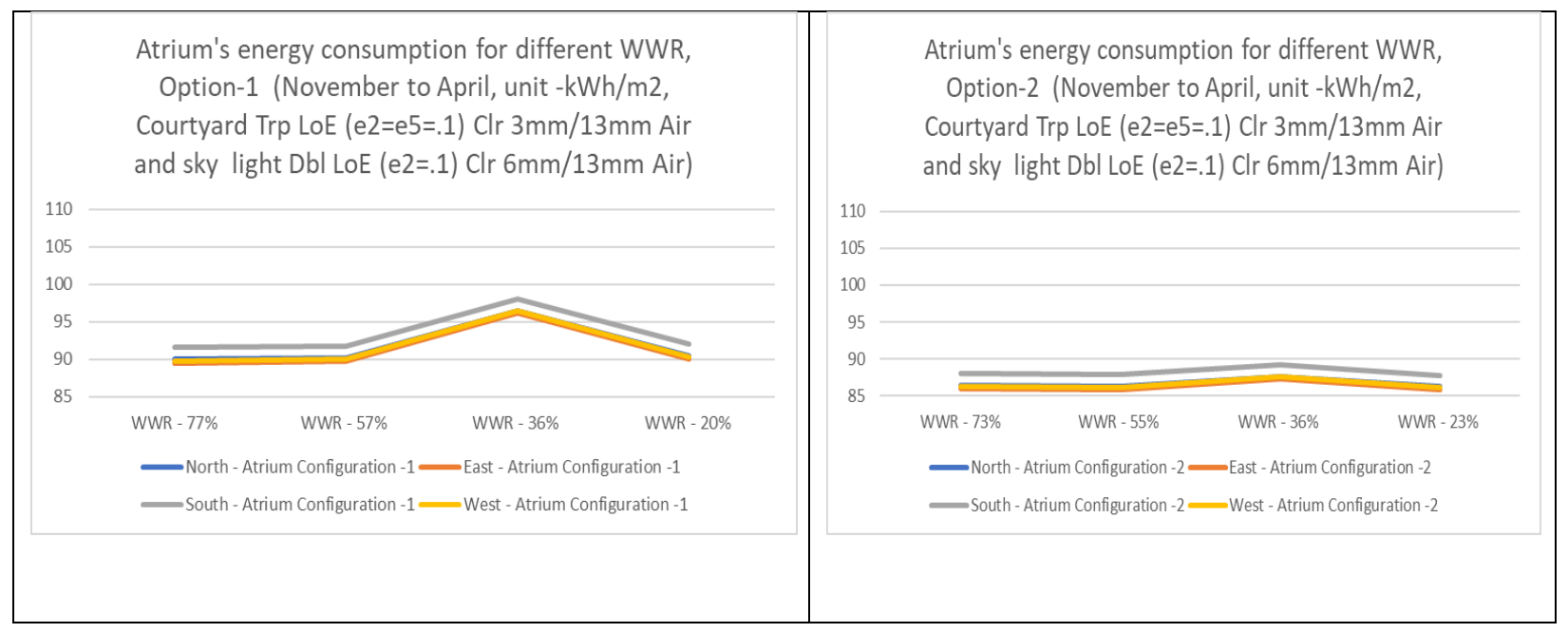

Appendix XXXV Energy consumption of atrium house with different orientation

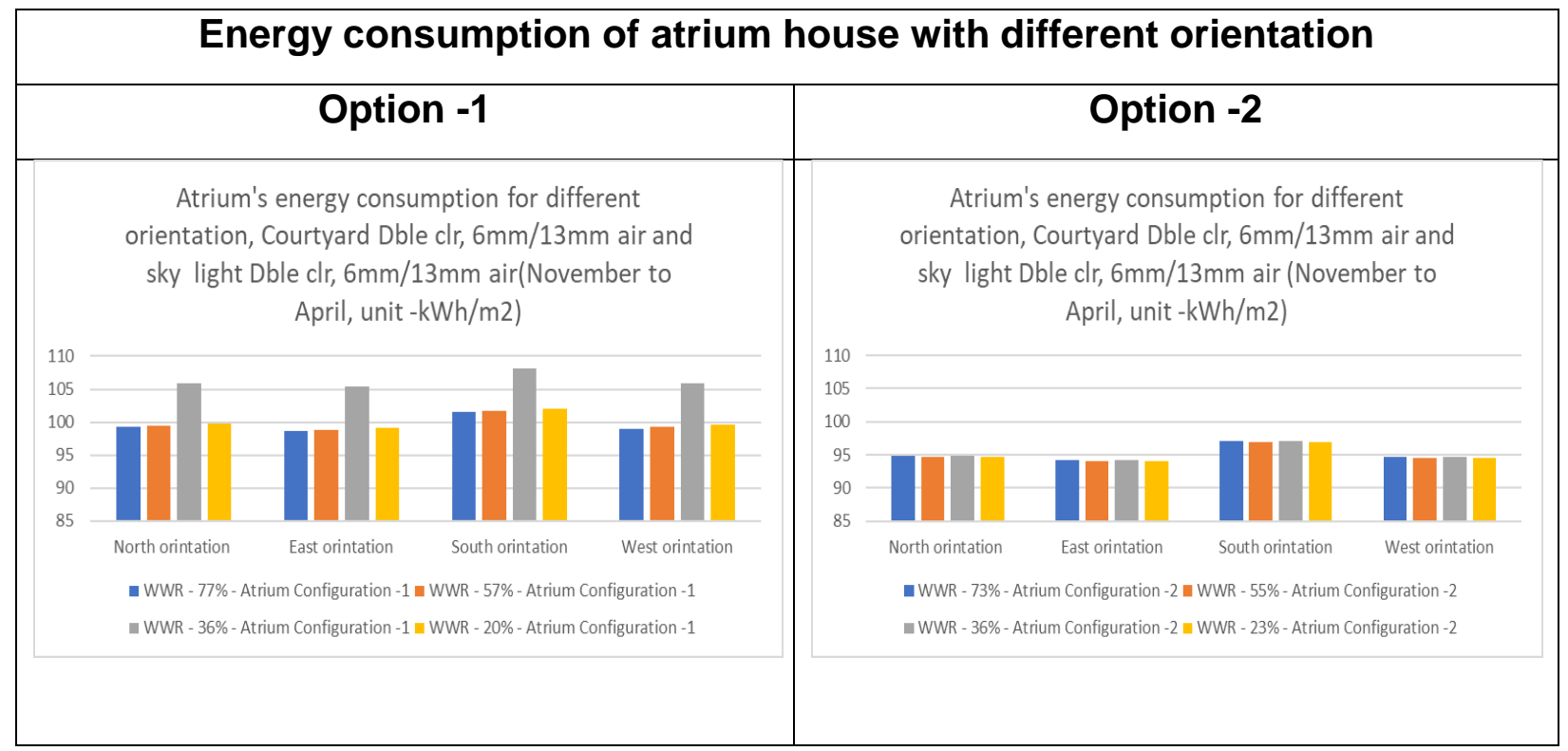




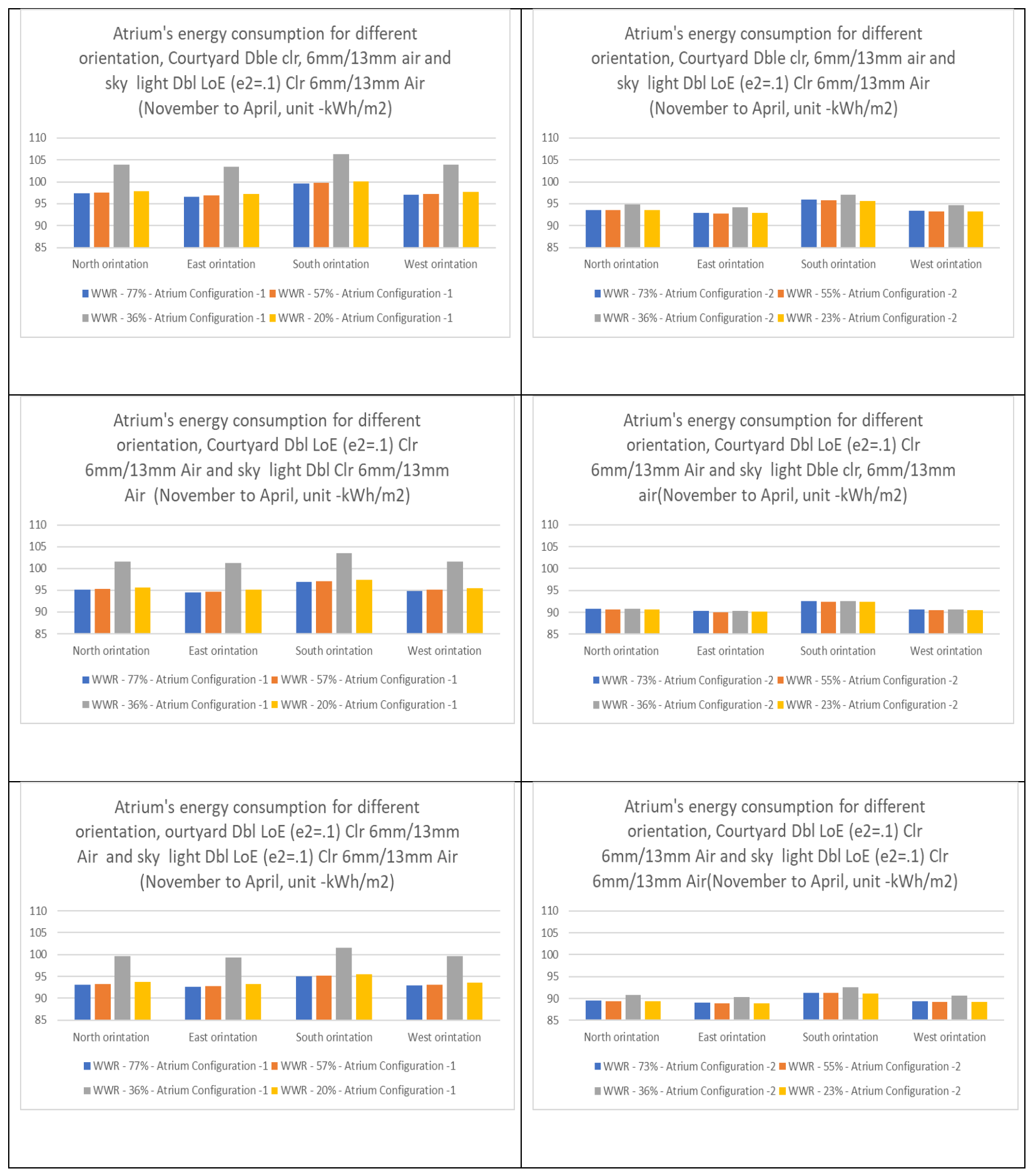




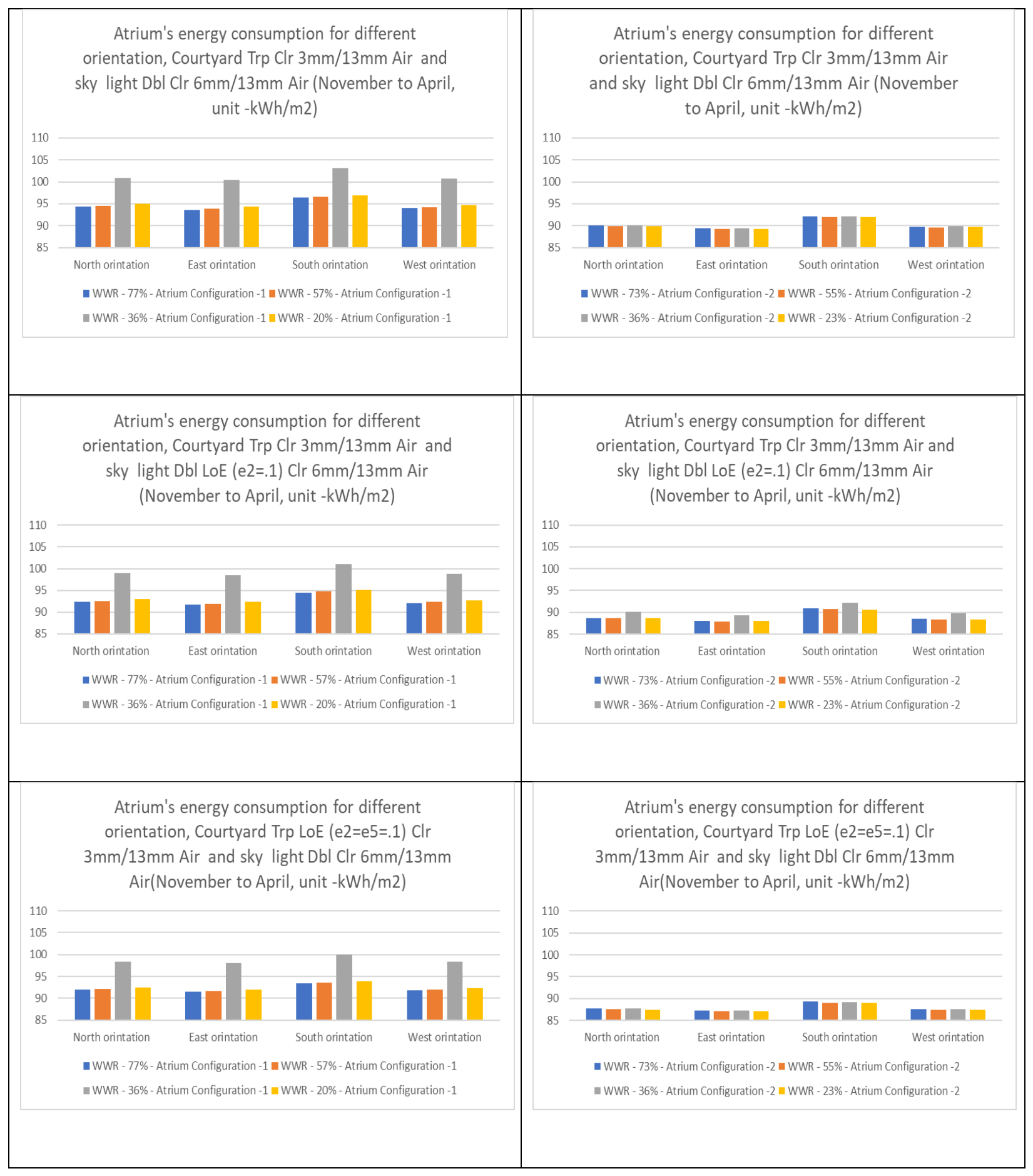




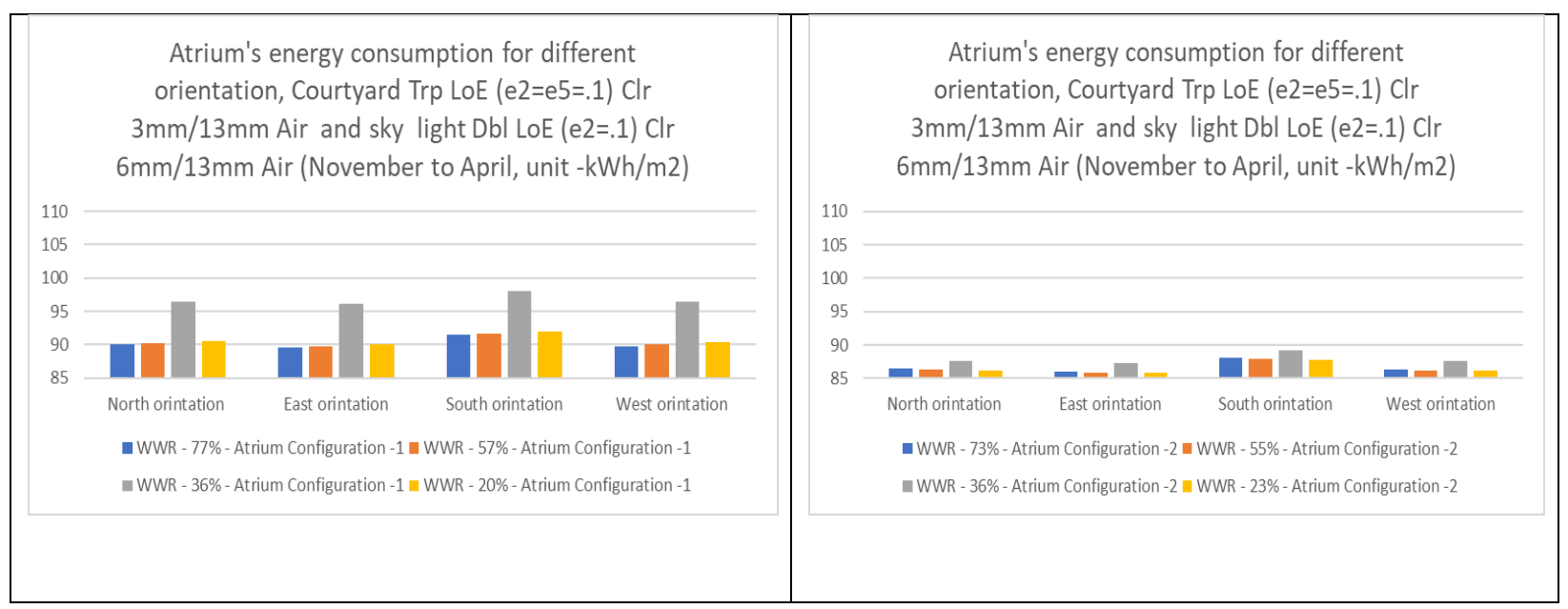




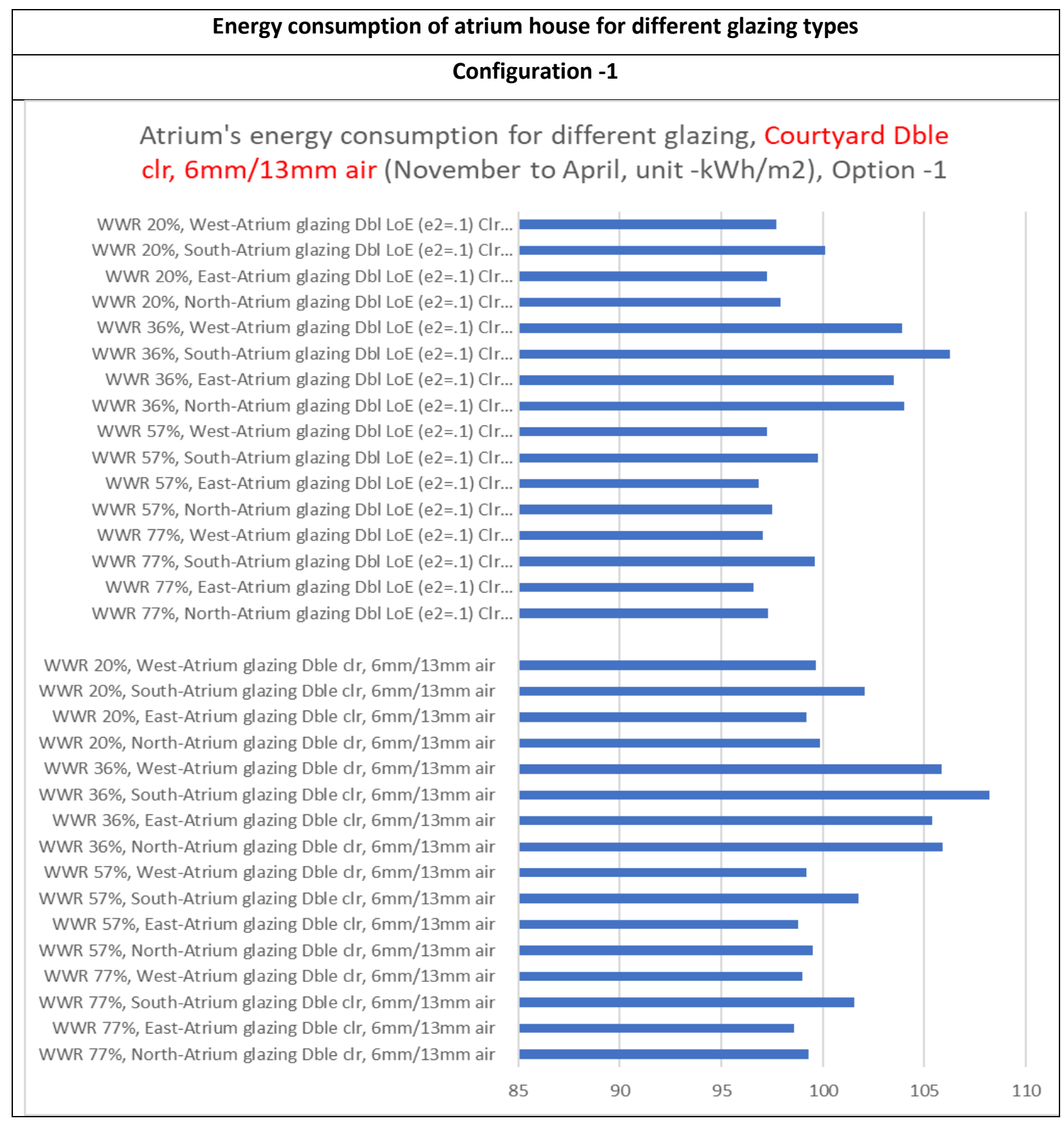




\section{Atrium's energy consumption for different glazing, Courtyard Dbl LoE (e2=.1) Clr 6mm/13mm Air (November to April, unit - $\mathrm{kWh} / \mathrm{m} 2$ ), Option -1}

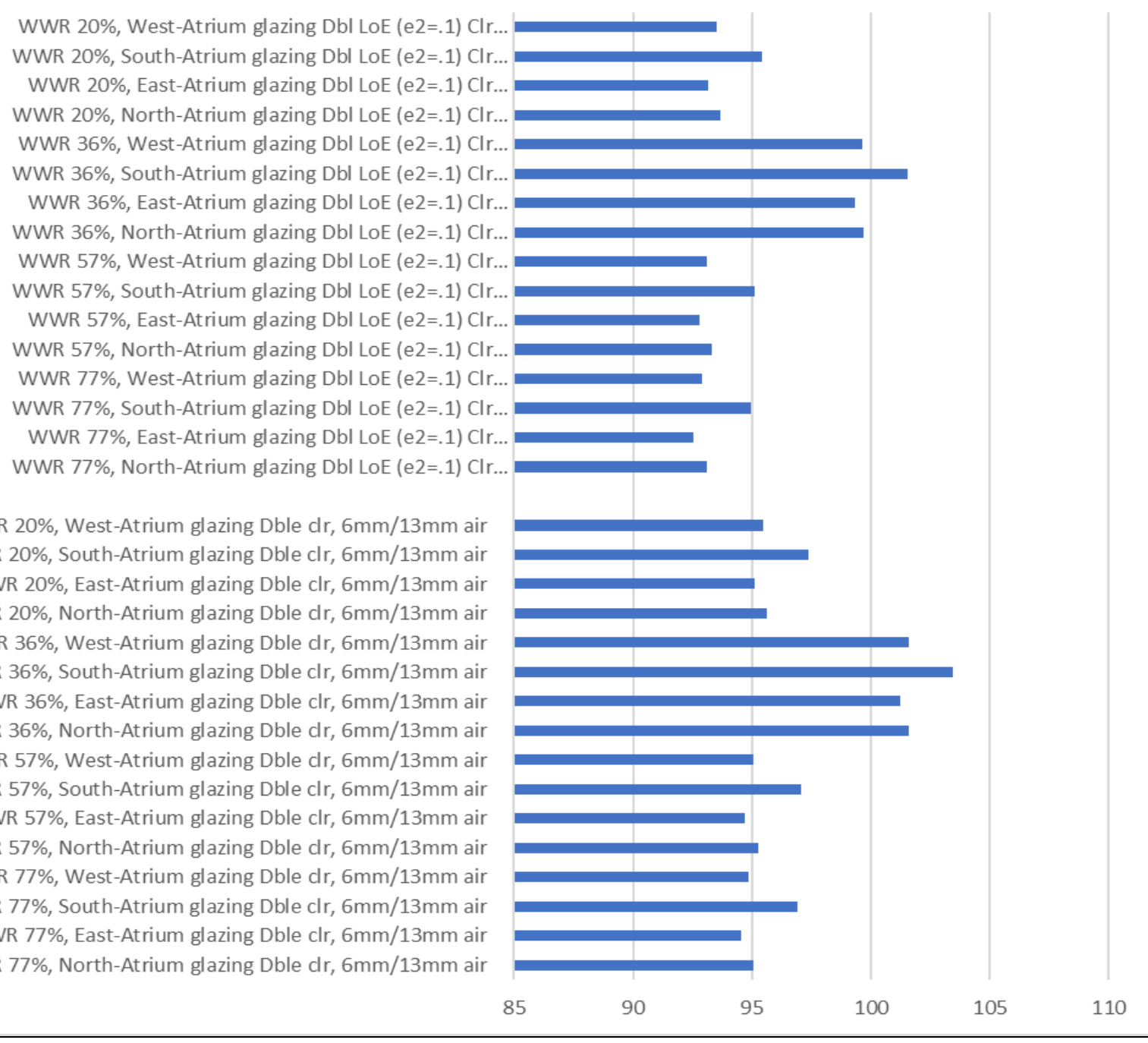




\section{Atrium's energy consumption for different glazing, Courtyard Trp $\mathrm{Clr} 3 \mathrm{~mm} / 13 \mathrm{~mm}$ Air (November to April, unit $-\mathrm{kWh} / \mathrm{m} 2$ ), Option -1}

WWR 20\%, West-Atrium glazing Dbl LoE (e2=.1) Clr... WWR $20 \%$, South-Atrium glazing Dbl LoE (e2=.1) Clr... WWR 20\%, East-Atrium glazing Dbl LoE (e2=.1) Clr.. WWR $20 \%$, North-Atrium glazing Dbl LoE (e2=.1) Clr.. WWR 36\%, West-Atrium glazing Dbl LoE (e2=.1) Clr.. WWR $36 \%$, South-Atrium glazing Dbl LoE $(\mathrm{e} 2=.1) \mathrm{Cl}$... WWR 36\%, East-Atrium glazing Dbl LoE (e2=.1) Clr... WWR 36\%, North-Atrium glazing Dbl LoE (e2=.1) Clr... WWR 57\%, West-Atrium glazing Dbl LoE (e2=.1) Clr... WWR 57\%, South-Atrium glazing Dbl LoE (e2=.1) Clr.. WWR 57\%, East-Atrium glazing Dbl LoE (e2=.1) Clr... WWR 57\%, North-Atrium glazing Dbl LoE (e2=.1) Clr.. WWR 77\%, West-Atrium glazing DbI LoE (e2=.1) Clr.. WWR 77\%, South-Atrium glazing Dbl LoE (e2=.1) Clr... WWR 77\%, East-Atrium glazing Dbl LoE (e2=.1) Clr... WWR 77\%, North-Atrium glazing Dbl LoE (e2=.1) Clr..

WWR 20\%, West-Atrium glazing Dble dr, $6 \mathrm{~mm} / 13 \mathrm{~mm}$ air WWR $20 \%$, South-Atrium glazing Dble clr, $6 \mathrm{~mm} / 13 \mathrm{~mm}$ air WWR $20 \%$, East-Atrium glazing Dble clr, $6 \mathrm{~mm} / 13 \mathrm{~mm}$ air WWR 20\%, North-Atrium glazing Dble clr, $6 \mathrm{~mm} / 13 \mathrm{~mm}$ air WWR $36 \%$, West-Atrium glazing Dble dr, $6 \mathrm{~mm} / 13 \mathrm{~mm}$ air WWR $36 \%$, South-Atrium glazing Dble clr, $6 \mathrm{~mm} / 13 \mathrm{~mm}$ air

WWR $36 \%$, East-Atrium glazing Dble clr, $6 \mathrm{~mm} / 13 \mathrm{~mm}$ air WWR $36 \%$, North-Atrium glazing Dble clr, $6 \mathrm{~mm} / 13 \mathrm{~mm}$ air WWR 57\%, West-Atrium glazing Dble dr, $6 \mathrm{~mm} / 13 \mathrm{~mm}$ air WWR 57\%, South-Atrium glazing Dble clr, $6 \mathrm{~mm} / 13 \mathrm{~mm}$ air WWR 57\%, East-Atrium glazing Dble clr, $6 \mathrm{~mm} / 13 \mathrm{~mm}$ air WWR 57\%, North-Atrium glazing Dble dr, $6 \mathrm{~mm} / 13 \mathrm{~mm}$ air WWR $77 \%$, West-Atrium glazing Dble dr, $6 \mathrm{~mm} / 13 \mathrm{~mm}$ air WWR 77\%, South-Atrium glazing Dble clr, $6 \mathrm{~mm} / 13 \mathrm{~mm}$ air WWR $77 \%$, East-Atrium glazing Dble clr, $6 \mathrm{~mm} / 13 \mathrm{~mm}$ air WWR $77 \%$, North-Atrium glazing Dble dr, $6 \mathrm{~mm} / 13 \mathrm{~mm}$ air

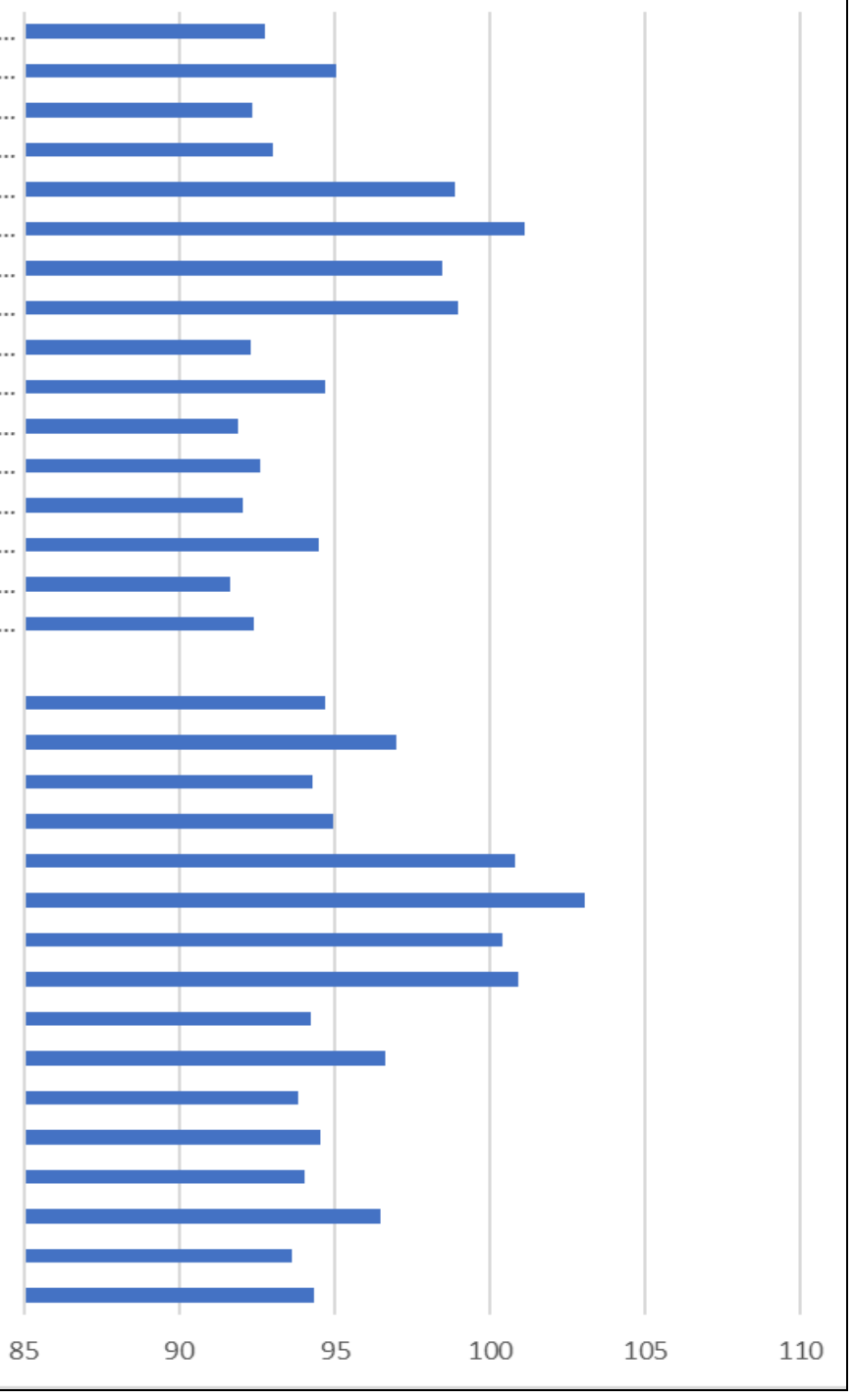




\section{Atrium's energy consumption for different glazing, Courtyard Trp LoE (e2=e5=.1) Clr 3mm/13mm Air (November to April, unit - $\mathrm{kWh} / \mathrm{m} 2$ ), Option -1}

WWR 20\%, West-Atrium glazing Dbl LoE (e2=.1) Clr... WWR $20 \%$, South-Atrium glazing Dbl LoE (e2=.1) Clr... WWR $20 \%$, East-Atrium glazing DbI LoE $(\mathrm{e} 2=.1) \mathrm{Cl}$... WWR $20 \%$, North-Atrium glazing Dbl LoE (e2=.1) Clr... WWR $36 \%$, West-Atrium glazing Dbl LoE $(\mathrm{e} 2=.1) \mathrm{Clr}$.. WWR $36 \%$, South-Atrium glazing Dbl LoE (e2=.1) Clr... WWR 36\%, East-Atrium glazing Dbl LoE (e2 $=.1) \mathrm{Clr}$.. WWR 36\%, North-Atrium glazing Dbl LoE (e2=.1) Clr... WWR 57\%, West-Atrium glazing Dbl LoE (e2=.1) Clr... WWR 57\%, South-Atrium glazing Dbl LoE (e2=.1) Clr... WWR 57\%, East-Atrium glazing Dbl LoE (e2=.1) Clr.. WWR 57\%, North-Atrium glazing Dbl LoE (e2=.1) Clr... WWR 77\%, West-Atrium glazing Dbl LoE (e2=.1) Clr... WWR 77\%, South-Atrium glazing Dbl LoE (e2=.1) Clr.. WWR 77\%, East-Atrium glazing Dbl LoE (e2=.1) Clr... WWR 77\%, North-Atrium glazing Dbl LoE (e2=.1) Clr...

WWR $20 \%$, West-Atrium glazing Dble $\mathrm{dr}, 6 \mathrm{~mm} / 13 \mathrm{~mm}$ air WWR $20 \%$, South-Atrium glazing Dble clr, $6 \mathrm{~mm} / 13 \mathrm{~mm}$ air WWR $20 \%$, East-Atrium glazing Dble clr, $6 \mathrm{~mm} / 13 \mathrm{~mm}$ air WWR $20 \%$, North-Atrium glazing Dble clr, $6 \mathrm{~mm} / 13 \mathrm{~mm}$ air WWR $36 \%$, West-Atrium glazing Dble $\mathrm{dr}, 6 \mathrm{~mm} / 13 \mathrm{~mm}$ air WWR $36 \%$, South-Atrium glazing Dble clr, $6 \mathrm{~mm} / 13 \mathrm{~mm}$ air WWR $36 \%$, East-Atrium glazing Dble clr, $6 \mathrm{~mm} / 13 \mathrm{~mm}$ air WWR $36 \%$, North-Atrium glazing Dble clr, $6 \mathrm{~mm} / 13 \mathrm{~mm}$ air WWR $57 \%$, West-Atrium glazing Dble $\mathrm{dr}, 6 \mathrm{~mm} / 13 \mathrm{~mm}$ air WWR 57\%, South-Atrium glazing Dble clr, $6 \mathrm{~mm} / 13 \mathrm{~mm}$ air WWR 57\%, East-Atrium glazing Dble clr, $6 \mathrm{~mm} / 13 \mathrm{~mm}$ air WWR 57\%, North-Atrium glazing Dble clr, $6 \mathrm{~mm} / 13 \mathrm{~mm}$ air WWR $77 \%$, West-Atrium glazing Dble clr, $6 \mathrm{~mm} / 13 \mathrm{~mm}$ air WWR 77\%, South-Atrium glazing Dble clr, $6 \mathrm{~mm} / 13 \mathrm{~mm}$ air

WWR $77 \%$, East-Atrium glazing Dble clr, $6 \mathrm{~mm} / 13 \mathrm{~mm}$ air WWR $77 \%$, North-Atrium glazing Dble dr, $6 \mathrm{~mm} / 13 \mathrm{~mm}$ air

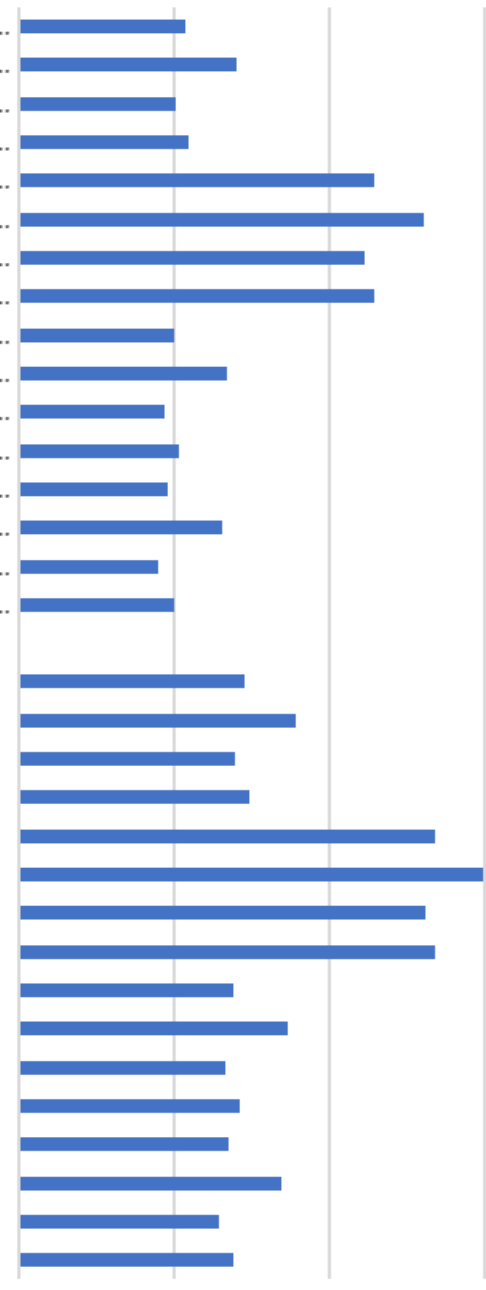




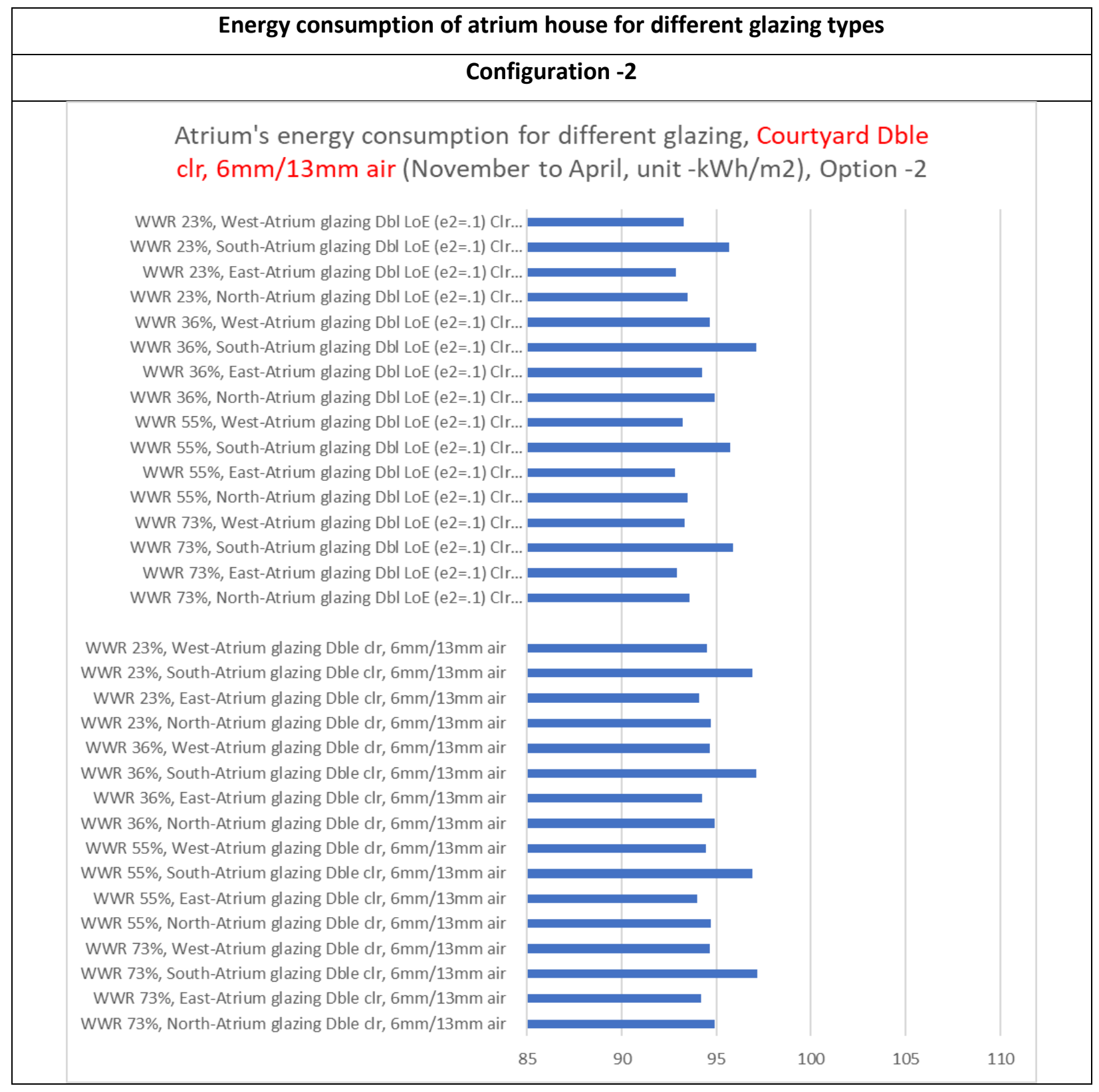




\section{Atrium's energy consumption for different glazing, Courtyard Dbl LoE (e2=.1) Clr 6mm/13mm Air (November to April, unit - $\mathrm{kWh} / \mathrm{m} 2)$, Option -2}

WWR 23\%, West-Atrium glazing Dbl LoE (e2=.1) Clr... WWR 23\%, South-Atrium glazing Dbl LoE (e2=.1) Clr... WWR 23\%, East-Atrium glazing Dbl LoE (e2=.1) Clr... WWR 23\%, North-Atrium glazing Dbl LoE (e2=.1) Clr... WWR 36\%, West-Atrium glazing Dbl LoE (e2=.1) Clr... WWR 36\%, South-Atrium glazing Dbl LoE (e2=.1) Clr..

WWR $36 \%$, East-Atrium glazing Dbl LoE (e2=.1) Clr... WWR 36\%, North-Atrium glazing Dbl LoE (e2=.1) Clr... WWR 55\%, West-Atrium glazing Dbl LoE (e2=.1) Clr... WWR 55\%, South-Atrium glazing Dbl LoE (e2=.1) Clr.. WWR 55\%, East-Atrium glazing Dbl LoE (e2=.1) Clr... WWR 55\%, North-Atrium glazing Dbl LoE (e2=.1) Clr... WWR 73\%, West-Atrium glazing Dbl LoE (e2=.1) Clr... WWR 73\%, South-Atrium glazing Dbl LoE (e2=.1) Clr.. WWR 73\%, East-Atrium glazing Dbl LoE (e2=.1) Clr.. WWR 73\%, North-Atrium glazing Dbl LoE (e2=.1) Clr..

WWR 23\%, West-Atrium glazing Dble clr, $6 \mathrm{~mm} / 13 \mathrm{~mm}$ air WWR 23\%, South-Atrium glazing Dble clr, $6 \mathrm{~mm} / 13 \mathrm{~mm}$ air WWR 23\%, East-Atrium glazing Dble clr, $6 \mathrm{~mm} / 13 \mathrm{~mm}$ air WWR 23\%, North-Atrium glazing Dble $\mathrm{dr}, 6 \mathrm{~mm} / 13 \mathrm{~mm}$ air WWR $36 \%$, West-Atrium glazing Dble dr, $6 \mathrm{~mm} / 13 \mathrm{~mm}$ air WWR $36 \%$, South-Atrium glazing Dble clr, $6 \mathrm{~mm} / 13 \mathrm{~mm}$ air WWR $36 \%$, East-Atrium glazing Dble clr, $6 \mathrm{~mm} / 13 \mathrm{~mm}$ air WWR $36 \%$, North-Atrium glazing Dble $\mathrm{dr}, 6 \mathrm{~mm} / 13 \mathrm{~mm}$ air WWR 55\%, West-Atrium glazing Dble $\mathrm{dr}, 6 \mathrm{~mm} / 13 \mathrm{~mm}$ air WWR 55\%, South-Atrium glazing Dble clr, $6 \mathrm{~mm} / 13 \mathrm{~mm}$ air WWR 55\%, East-Atrium glazing Dble clr, $6 \mathrm{~mm} / 13 \mathrm{~mm}$ air WWR 55\%, North-Atrium glazing Dble $\mathrm{dr}, 6 \mathrm{~mm} / 13 \mathrm{~mm}$ air WWR 73\%, West-Atrium glazing Dble dr, $6 \mathrm{~mm} / 13 \mathrm{~mm}$ air WWR $73 \%$, South-Atrium glazing Dble clr, $6 \mathrm{~mm} / 13 \mathrm{~mm}$ air WWR 73\%, East-Atrium glazing Dble clr, $6 \mathrm{~mm} / 13 \mathrm{~mm}$ air WWR 73\%, North-Atrium glazing Dble dr, $6 \mathrm{~mm} / 13 \mathrm{~mm}$ air

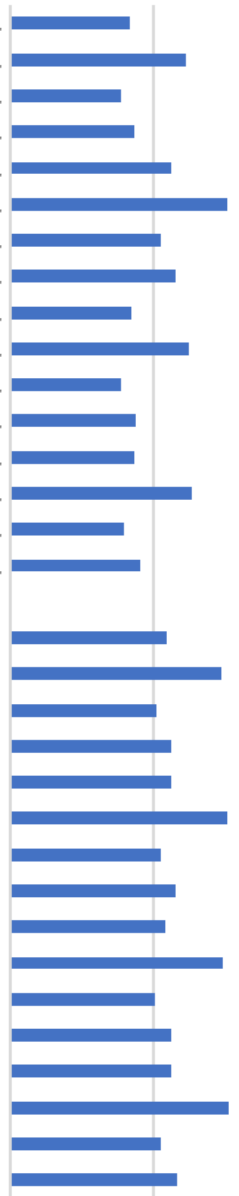

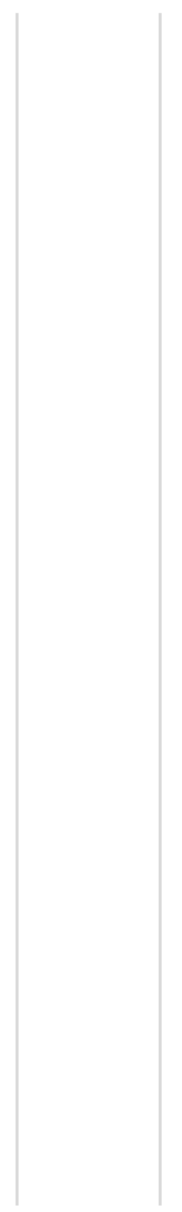

95

100

105

110 


\section{Atrium's energy consumption for different glazing, Courtyard Trp Clr 3mm/13mm Air (November to April, unit -kWh/m2), Option -2}

WWR 23\%, West-Atrium glazing Dbl LoE (e2=.1) Clr... WWR 23\%, South-Atrium glazing Dbl LoE (e2=.1) Clr... WWR 23\%, East-Atrium glazing Dbl LoE (e2=.1) Clr... WWR 23\%, North-Atrium glazing Dbl LoE (e2=.1) Clr... WWR 36\%, West-Atrium glazing Dbl LoE (e2=.1) Clr... WWR 36\%, South-Atrium glazing Dbl LoE (e2=.1) Clr...

WWR 36\%, East-Atrium glazing Dbl LoE (e2=.1) Clr... WWR 36\%, North-Atrium glazing Dbl LoE (e2=.1) Clr.. WWR 55\%, West-Atrium glazing Dbl LoE (e2=.1) Clr.. WWR 55\%, South-Atrium glazing Dbl LoE (e2=.1) Clr..

WWR 55\%, East-Atrium glazing Dbl LoE $(\mathrm{e} 2=.1) \mathrm{Clr}$... WWR 55\%, North-Atrium glazing Dbl LoE (e2=.1) Clr.. WWR 73\%, West-Atrium glazing Dbl LoE (e2=.1) Clr... WWR 73\%, South-Atrium glazing Dbl LoE (e2=.1) Clr...

WWR 73\%, East-Atrium glazing Dbl LoE $(e 2=.1) \mathrm{Cl}$ WWR 73\%, North-Atrium glazing Dbl LoE (e2=.1) Clr...

WWR 23\%, West-Atrium glazing Dble $\mathrm{dr}, 6 \mathrm{~mm} / 13 \mathrm{~mm}$ air WWR 23\%, South-Atrium glazing Dble clr, $6 \mathrm{~mm} / 13 \mathrm{~mm}$ air WWR 23\%, East-Atrium glazing Dble clr, $6 \mathrm{~mm} / 13 \mathrm{~mm}$ air WWR 23\%, North-Atrium glazing Dble $\mathrm{dr}, 6 \mathrm{~mm} / 13 \mathrm{~mm}$ air WWR 36\%, West-Atrium glazing Dble dr, $6 \mathrm{~mm} / 13 \mathrm{~mm}$ air WWR 36\%, South-Atrium glazing Dble clr, $6 \mathrm{~mm} / 13 \mathrm{~mm}$ air WWR $36 \%$, East-Atrium glazing Dble clr, $6 \mathrm{~mm} / 13 \mathrm{~mm}$ air WWR 36\%, North-Atrium glazing Dble $\mathrm{dr}, 6 \mathrm{~mm} / 13 \mathrm{~mm}$ air WWR 55\%, West-Atrium glazing Dble $\mathrm{dr}, 6 \mathrm{~mm} / 13 \mathrm{~mm}$ air WWR 55\%, South-Atrium glazing Dble clr, $6 \mathrm{~mm} / 13 \mathrm{~mm}$ air WWR 55\%, East-Atrium glazing Dble clr, $6 \mathrm{~mm} / 13 \mathrm{~mm}$ air WWR 55\%, North-Atrium glazing Dble $\mathrm{dr}, 6 \mathrm{~mm} / 13 \mathrm{~mm}$ air WWR 73\%, West-Atrium glazing Dble $\mathrm{dr}, 6 \mathrm{~mm} / 13 \mathrm{~mm}$ air WWR 73\%, South-Atrium glazing Dble clr, $6 \mathrm{~mm} / 13 \mathrm{~mm}$ air WWR 73\%, East-Atrium glazing Dble clr, $6 \mathrm{~mm} / 13 \mathrm{~mm}$ air WWR 73\%, North-Atrium glazing Dble dr, $6 \mathrm{~mm} / 13 \mathrm{~mm}$ air
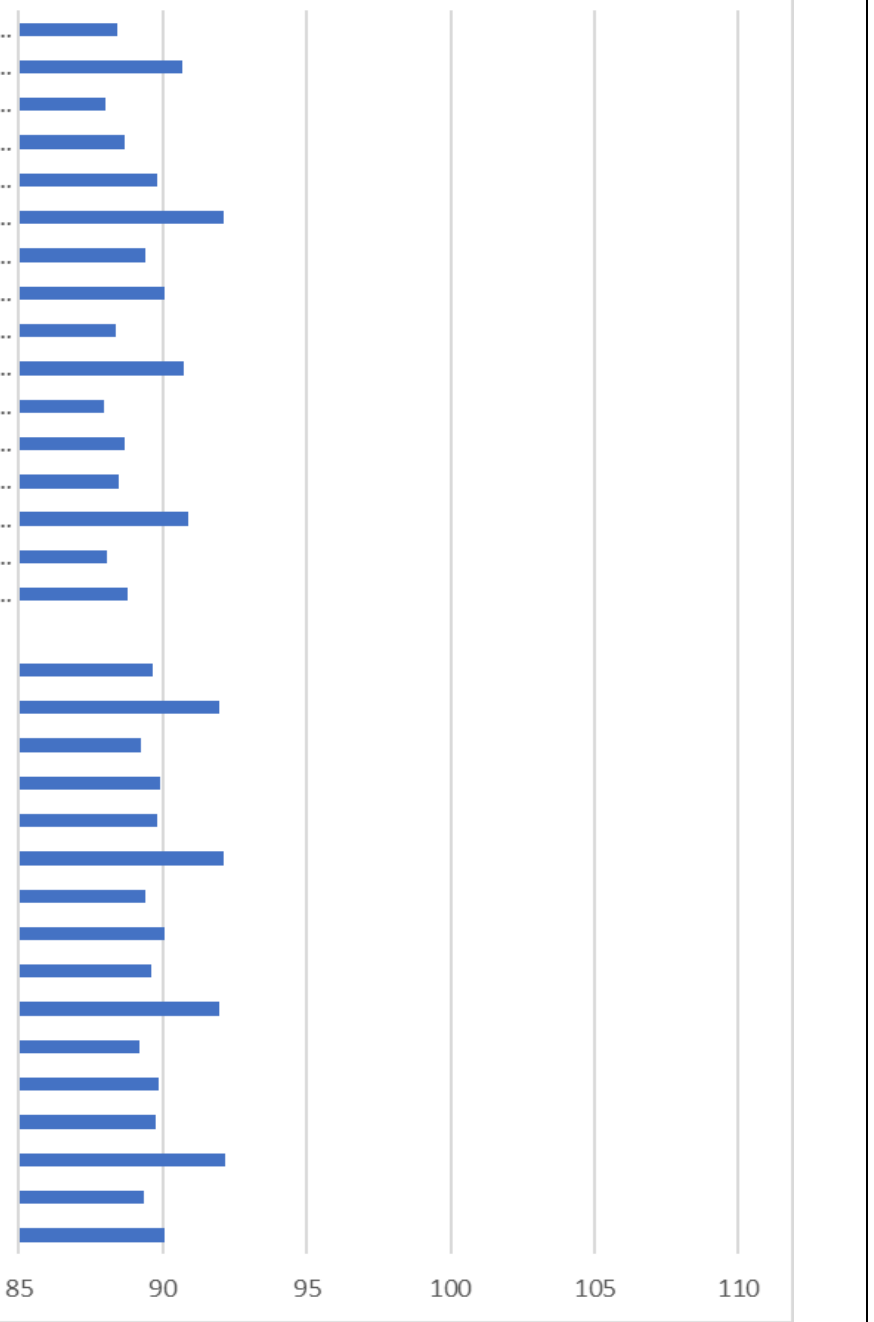


\section{Atrium's energy consumption for different glazing, Courtyard Trp LoE (e2=e5=.1) Clr 3mm/13mm Air (November to April, unit - $\mathrm{kWh} / \mathrm{m} 2)$, Option -2}

WWR 23\%, West-Atrium glazing Dbl LoE (e2=.1) Clr... WWR 23\%, South-Atrium glazing Dbl LoE (e2=.1) Clr... WWR 23\%, East-Atrium glazing Dbl LoE (e2=.1) Clr... WWR 23\%, North-Atrium glazing Dbl LoE (e2=.1) Clr... WWR 36\%, West-Atrium glazing Dbl LoE (e2=.1) Clr... WWR 36\%, South-Atrium glazing Dbl LoE (e2=.1) Clr... WWR 36\%, East-Atrium glazing DbI LoE (e2=.1) Clr... WWR 36\%, North-Atrium glazing Dbl LoE (e2=.1) Clr... WWR 55\%, West-Atrium glazing Dbl LoE (e2=.1) Clr.. WWR 55\%, South-Atrium glazing Dbl LoE (e2=1) Clr. WWR 55\%, East-Atrium glazing Dbl LoE (e2=.1) Clr.. WWR 55\%, North-Atrium glazing Dbl LoE (e2=.1) Clr... WWR 73\%, West-Atrium glazing Dbl LoE (e2=.1) Clr... WWR 73\%, South-Atrium glazing Dbl LoE (e2=.1) Clr WWR 73\%, East-Atrium glazing Dbl LoE (e2=.1) Clr.. WWR 73\%, North-Atrium glazing Dbl LoE (e2=.1) Clr..

WWR 23\%, West-Atrium glazing Dble dr, $6 \mathrm{~mm} / 13 \mathrm{~mm}$ air WWR 23\%, South-Atrium glazing Dble clr, $6 \mathrm{~mm} / 13 \mathrm{~mm}$ air WWR $23 \%$, East-Atrium glazing Dble clr, $6 \mathrm{~mm} / 13 \mathrm{~mm}$ air WWR 23\%, North-Atrium glazing Dble $\mathrm{dr}, 6 \mathrm{~mm} / 13 \mathrm{~mm}$ air WWR $36 \%$, West-Atrium glazing Dble dr, $6 \mathrm{~mm} / 13 \mathrm{~mm}$ air WWR 36\%, South-Atrium glazing Dble clr, $6 \mathrm{~mm} / 13 \mathrm{~mm}$ air WWR $36 \%$, East-Atrium glazing Dble clr, $6 \mathrm{~mm} / 13 \mathrm{~mm}$ air WWR 36\%, North-Atrium glazing Dble dr, $6 \mathrm{~mm} / 13 \mathrm{~mm}$ air WWR 55\%, West-Atrium glazing Dble $\mathrm{dr}, 6 \mathrm{~mm} / 13 \mathrm{~mm}$ air WWR 55\%, South-Atrium glazing Dble clr, $6 \mathrm{~mm} / 13 \mathrm{~mm}$ air WWR 55\%, East-Atrium glazing Dble clr, $6 \mathrm{~mm} / 13 \mathrm{~mm}$ air WWR 55\%, North-Atrium glazing Dble dr, $6 \mathrm{~mm} / 13 \mathrm{~mm}$ air WWR 73\%, West-Atrium glazing Dble dr, $6 \mathrm{~mm} / 13 \mathrm{~mm}$ air WWR 73\%, South-Atrium glazing Dble clr, $6 \mathrm{~mm} / 13 \mathrm{~mm}$ air WWR 73\%, East-Atrium glazing Dble clr, $6 \mathrm{~mm} / 13 \mathrm{~mm}$ air WWR 73\%, North-Atrium glazing Dble dr, $6 \mathrm{~mm} / 13 \mathrm{~mm}$ air

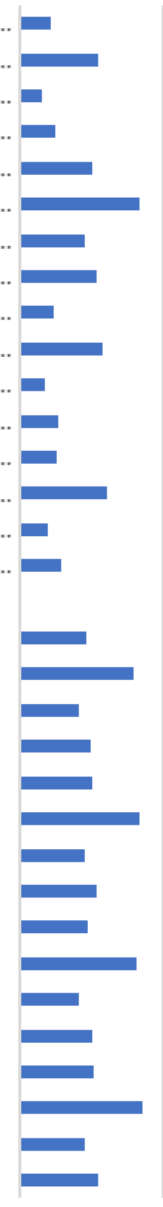

$85-90$

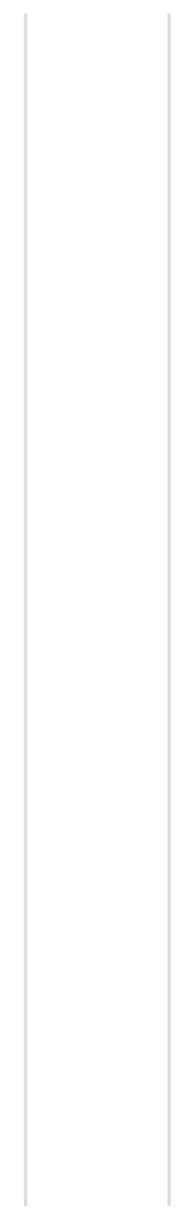

$95 \quad 100$

105

110 


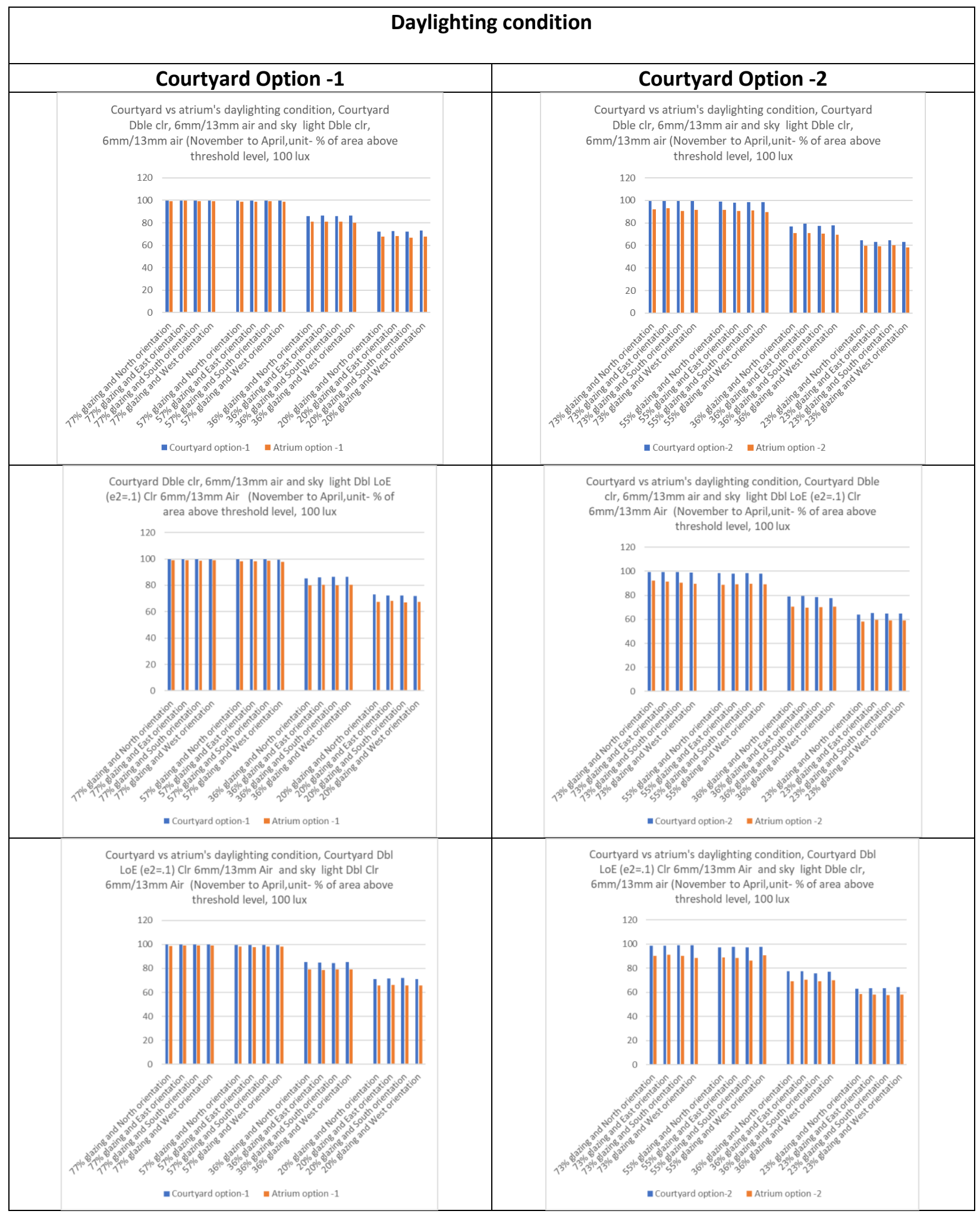




\begin{tabular}{|c|c|}
\hline $\begin{array}{l}\text { Courtyard vs atrium's daylighting condition, Courtyard Dbl } \\
\text { LoE (e2=.1) Clr } 6 \mathrm{~mm} / 13 \mathrm{~mm} \text { Air and sky light Db LoE } \\
\text { (e2=.1) Clr } 6 \mathrm{~mm} / 13 \mathrm{~mm} \text { Air (November to April,unit-\% of } \\
\text { area above threshold level, } 100 \text { lux }\end{array}$ & 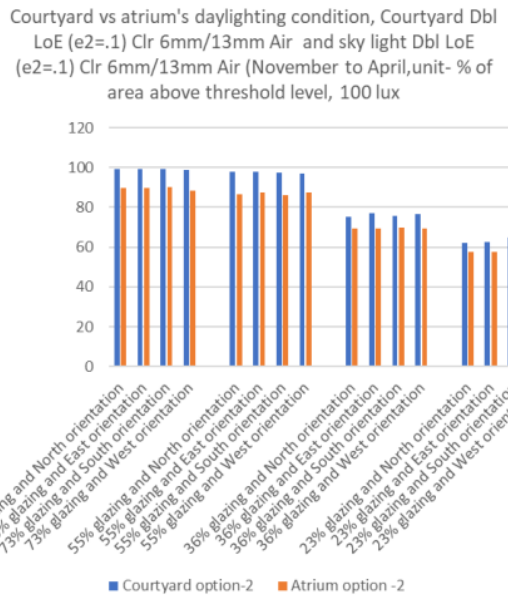 \\
\hline $\begin{array}{l}\text { Courtyard vs atrium's daylighting condition, Courtyard Trp } \\
\text { Clr 3mm/13mm Air and sky light Db Cla } 6 \mathrm{~mm} / 13 m \text { m Air } \\
\text { (November to April,unit- \% of area above threshold level, } \\
\text { (No lux }\end{array}$ & 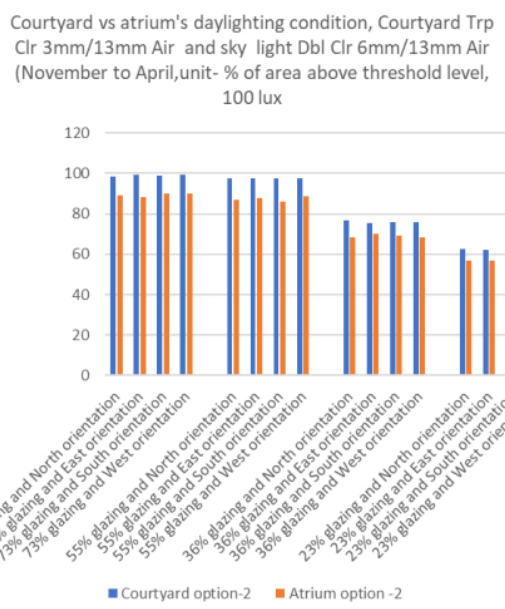 \\
\hline $\begin{array}{l}\text { Courtyard vs atrium's daylighting condition, Courtyard Trp } \\
\text { Clr } 3 \mathrm{~mm} / 13 \mathrm{~mm} \text { Air and sky light Dbl Le (e2=.1) Clr } \\
6 \mathrm{~mm} / 13 \mathrm{~mm} \text { Air (November to April,unito \% of area above } \\
\text { threshold level, } 100 \text { lux }\end{array}$ & $\begin{array}{l}\text { Courtyard vs atrium's daylighting condition, Courtyard Trp } \\
\text { Clr 3mm/13mm Air and sky light Dbl LoE (e2=1) Clr } \\
6 \mathrm{~mm} / 13 \mathrm{~mm} \text { Air(November to April,unit- \% of area above } \\
\text { threshold level, } 100 \text { lux }\end{array}$ \\
\hline
\end{tabular}




\begin{tabular}{|c|c|}
\hline $\begin{array}{l}\text { Courtyard vs atrium's daylighting condition, Courtyard Trp } \\
\text { LoE (e2=e5=.1) Clr 3mm/13mm Air and sky light Dbl Clr } \\
6 \mathrm{~mm} / 13 \mathrm{~mm} \text { Air (November to April,unit- \% of area above } \\
\text { threshold level, } 100 \text { lux }\end{array}$ & $\begin{array}{l}\text { Courtyard vs atrium's daylighting condition, (November } \\
\text { tCourtyard Trp LoE (e2=e5=.1) Clr } 3 \mathrm{~mm} / 13 \mathrm{~mm} \text { Air and sky } \\
\text { light Dbl Clr } 6 \mathrm{~mm} / 13 \mathrm{~mm} \text { Airo April,unit-\% of area above } \\
\text { threshold level, } 100 \text { lux }\end{array}$ \\
\hline $\begin{array}{l}\text { Courtyard vs atrium's daylighting condition, Courtyard Trp } \\
\text { LoE (e2=e5=.1) Clr } 3 \mathrm{~mm} / 13 \mathrm{~mm} \text { Air and sky light Dbl LoE } \\
(\mathrm{e} 2=.1) \mathrm{Clr} 6 \mathrm{~mm} / 13 \mathrm{~mm} \text { Air (November to April,unit-\% of } \\
\text { area above threshold level, } 100 \text { lux }\end{array}$ & $\begin{array}{l}\text { Courtyard vs atrium's daylighting condition, Courtyard Trp } \\
\text { LoE (e2=e5=.1) Clr } 3 \mathrm{~mm} / 13 \mathrm{~mm} \text { Air and sky light Dbl LoE } \\
(\mathrm{e} 2=.1) \mathrm{Cl} 6 \mathrm{~mm} / 13 \mathrm{~mm} \text { Air (November to April,unit-\% of } \\
\text { area above threshold level, } 100 \text { lux }\end{array}$ \\
\hline
\end{tabular}


Appendix XXXVIII Daylighting condition plotted for courtyard glazing Double LoE (e2=.1) Clr 6mm/13mm Air and sky light Double Clear 6mm/13mm Air in configuration-1

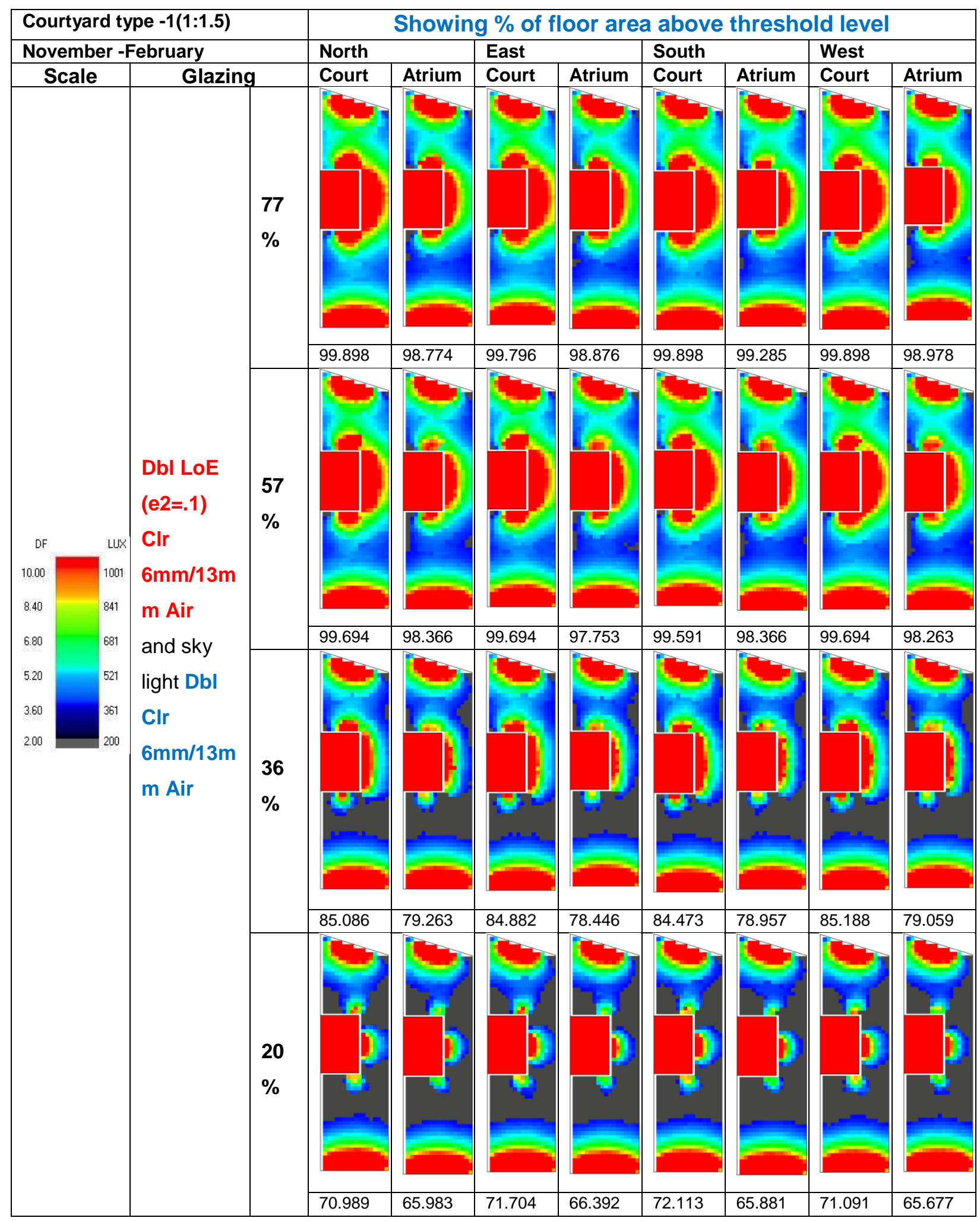


Appendix XXXIX Normalization and weighted value of combinations

\begin{tabular}{|c|c|c|c|c|c|c|c|c|c|c|c|c|c|}
\hline \multirow[t]{2}{*}{ Options } & \multicolumn{3}{|c|}{$\begin{array}{l}\text { Areae A: Energy consumtion } \\
\text { reduction compared o courtyard } \\
\quad \text { (Prioritity alue 2) }\end{array}$} & \multicolumn{3}{|c|}{\begin{tabular}{|l} 
Area B: Energy consumption by \\
atrium option (Priority value 2)
\end{tabular}} & \multicolumn{3}{|c|}{ 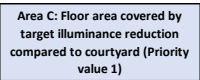 } & \multicolumn{3}{|c|}{$\begin{array}{l}\text { Area D: Floor area covered by } \\
\text { target liluminance (Priority value } 1\end{array}$} & $\begin{array}{l}\text { Neighted } \\
\text { totat }\end{array}$ \\
\hline & & Normali: & & & & (2) & & Normalize & Weighted & $\begin{array}{l}\text { ercentage } \\
\text { of floor }\end{array}$ & & & \\
\hline & & value & & & ed value & value & & d value & value & area & value & value & \\
\hline 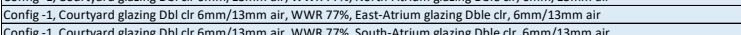 & $\frac{14.5}{14.5}$ & 6.4 & $\frac{12.9}{12.9}$ & 98.6 & 4.3 & 8.6 & $\frac{0.3}{0.3}$ & $\frac{10.0}{10.0}$ & 10.0 & 99.5 & 10.0 & $\frac{19.0}{10.0}$ & 41.5. \\
\hline & $\frac{15.5}{15.5} \mathrm{C}$ & & & 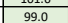 & & & & & & & & & $\begin{array}{lll}35.0 \\
41.8\end{array}$ \\
\hline 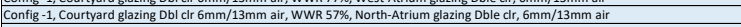 & 16.7 & 7.6 & 15.2 & 99.5 & ${ }_{3.9}$ & 7.8 & $\frac{1.1}{1.1}$ & 9.6 & 9.6 & 98.7 & 9.8 & 9.8 & 42.6 \\
\hline 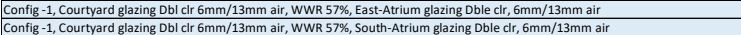 & $\frac{\frac{12.3}{10.0}}{10.0}$ & $\frac{5.3}{4.1}$ & $\frac{10.6}{8.1}$ & $\frac{98.8}{10.7}$ & $\frac{4.2}{2.9}$ & $\frac{8.4}{5.8}$ & $\frac{1.2}{0.6}$ & $\frac{9.6}{9.9}$ & $\frac{9.6}{9.9}$ & 98.7 & 9.8 & $\frac{9.8}{9.9}$ & $\frac{38.4}{33.7}$ \\
\hline 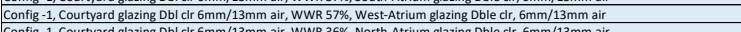 & 13.2 & 5.8 & 11.5 & 99.2 & 4.0 & 8.0 & 1.0 & 9.7 & 9.7 & 98.8 & 9.8 & 9.8 & \\
\hline 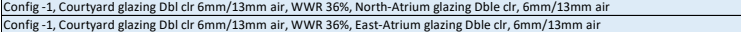 & & & $\frac{13.4}{1.3 .}$ & $\frac{105.9}{105.4}$ & 1.0 & & 5.9 & 7.5 & & 80.9 & 6.0 & 6.0 & 28.9 \\
\hline 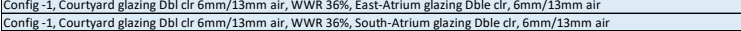 & $\frac{14.8}{9.5}$ & $\frac{6.6}{3.8}$ & $\frac{13.2}{7.7}$ & $\frac{105.4}{108.2}$ & $\frac{1.2}{0.0}$ & 2.5 & $\frac{6.2}{5.9}$ & 7.45 & $\frac{7.4}{7.5}$ & 80.9 & $\frac{6.0}{6.0}$ & 6.0 & $\frac{29.1}{21.2}$ \\
\hline 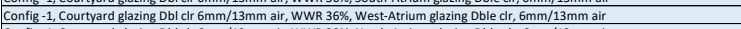 & 9.8 & 4.0 & 7.9 & 105.9 & 1.1 & 2.1 & 7.8 & 6.7 & 6.7 & 79.9 & 5.8 & 5.8 & \\
\hline 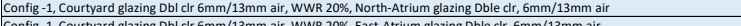 & 11.2 & 4.7 & 9.4 & 99.99 & 3.7 & 7.5 & 6.2 & 7.4 & 7.4 & 67.7 .7 & 3.1. & 3.1 & 27.4 \\
\hline$\frac{1 \mathrm{lct} 6 \mathrm{~mm} / 13 \mathrm{mmm} \text { alr, WWR } 20}{1 \mathrm{cl} 6 \mathrm{~mm} / 13 \mathrm{~mm} \text { air, WWR } 20}$ & $\frac{9.1}{9.5}$ & $\frac{3.6}{3.8}$ & $\frac{7.2}{7.6}$ & 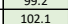 & $\begin{array}{l}\frac{20}{2.7} \\
2\end{array}$ & $\frac{8.0}{5.5}$ & $\frac{7.8}{7.2}$ & $\frac{7.60}{7.0}$ & $\frac{1.0}{7.0}$ & 668.9 & $\frac{3.2}{2.9}$ & $\frac{3.2}{2.9}$ & 26.1 \\
\hline Ict $6 \mathrm{~mm} / 13 \mathrm{~mm}$ air, WWR 20 & 10.0 & & & 99.6 & 3.8 & & 7.0 & 7.1 & & & & & \\
\hline 1, Courtyard glazing Dbl cl $6 \mathrm{~mm} / 13 \mathrm{~mm}$ air, WWR 77 & 21.2 & 9.9 & 19.8 & 97.3 & 4.9 & 9.7 & 0.9 & 9.7 & 9.7 & & 9.9 & & 49.2 \\
\hline 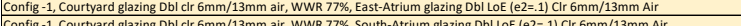 & $\frac{16.3}{129}$ & $\frac{7.3}{56}$ & $\frac{14.7}{1.12}$ & 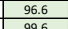 & 5.2 & 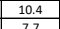 & 0.9 & 9.7 & 9.7 & 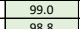 & 9.9 & 9.9 & $\frac{44.7}{3.9}$ \\
\hline 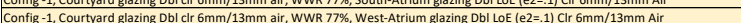 & & $\frac{1.0}{7.8}$ & 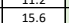 & $\begin{array}{l}390 \\
97.0\end{array}$ & & 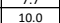 & $\frac{1.9}{0.9}$ & $\frac{9.7}{9.7}$ & & 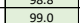 & & & \\
\hline 6, North-Atrium glazing D & (n) & & & 075 & 4.8 & & 18 & & & & & 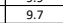 & \\
\hline 6, East-Atrium glazing Db LoE (e2)=1. & & 6.2 & 12.4 & 96.8 & & 10.2 & 1.4 & 9.5 & & 98.4 & & & \\
\hline 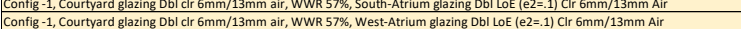 & $\frac{11.79}{14.9}$ & $\frac{5.0}{6.6}$ & $\frac{9.9}{13.3}$ & 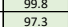 & $\frac{3.8}{4.9}$ & 7.5 & $\frac{1.3}{1.6}$ & $\frac{9.6}{9.4}$ & 9.6 & 98.0 & 9.8 & 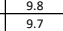 & $\frac{36.8}{42.1}$ \\
\hline 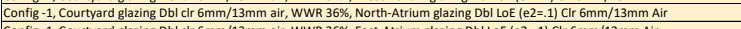 & 16.6 & 7.5 & 15.0 & 104.0 & 1.9 & 3.8 & 6.0 & 7.5 & 7.5 & 80.0 & 5.8 & 5.8 & 32.1 \\
\hline 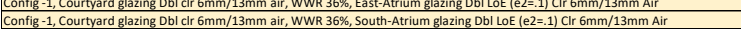 & $\frac{16.4}{11.1}$ & $\frac{7.4}{4.7}$ & $\frac{19.8}{9.3}$ & $\frac{103.5}{106.3}$ & $\frac{2.1}{0.9}$ & $\frac{4.2}{1.7}$ & $\frac{6.8}{7.3}$ & $\frac{7.2}{6.9}$ & $\frac{7.2}{6.9}$ & $\frac{80.4}{80.1}$ & 5.9 & $\frac{5.9}{5.8}$ & 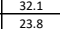 \\
\hline 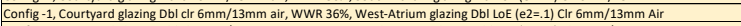 & & & & 103.9 & 1.9 & 3.8 & & & & 80.5 & 5.9 & 5.9 & \\
\hline 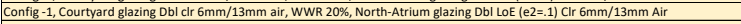 & & & 11.2 & 97.9 & 4.6. & & 7.8 & 6.7 & 6.7 & & & & 30.1 \\
\hline 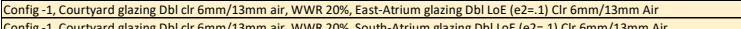 & 10.9 & $\frac{4.5}{4.5}$ & 9.1 & 97.3 & $\frac{4.9}{3.5}$ & $\frac{9.8}{72}$ & 5.4 & 7.8 & 7.8 & 68.3 & 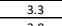 & 3.3 & 29.9 \\
\hline 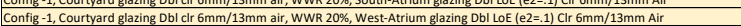 & 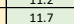 & 5.0 & 9.9. & 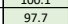 & $\begin{array}{l}3.0 \\
4.7\end{array}$ & 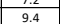 & 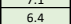 & 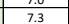 & 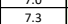 & 67.4 & $\begin{array}{l}3.1 \\
3.1\end{array}$ & 3.1 & \\
\hline , Courtyard Glazing Dblcr $6 \mathrm{~mm} / 13 \mathrm{~mm}$ air, WWR $73 \%$,North-Atrium Glazing Dble clt, $6 \mathrm{~mm} / 13 \mathrm{~mm}$ air & & & & & & & & & & & & & \\
\hline 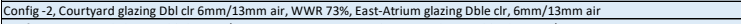 & 16.9 & 7.7 & 15.3 & 94.2 & 6.3 & 12.5 & 6.3 & 7.4 & 7.4 & 93.0 & 8.6 & 8.6 & 43.8 \\
\hline 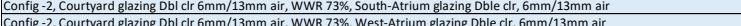 & $\frac{15.5}{17.6}$ & $\frac{7.0}{80}$ & $\frac{13.9}{16.1}$ & 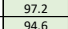 & $\frac{4.9}{61}$ & $\frac{9.9}{121}$ & $\frac{8.5}{77}$ & 6.4 & 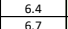 & $\frac{90.8}{917}$ & $\frac{8.1}{8.3}$ & $\frac{8.1}{8.3}$ & 38.3 \\
\hline 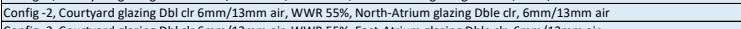 & 18.4 & 8.4 & 16.9 & 94.7 & 6.0 & 12.1 & $\frac{7.5}{7.5}$ & 6.8 & 6.8 & 91.6 & $\frac{8.3}{8.3}$ & 8.3 & 44.1 \\
\hline 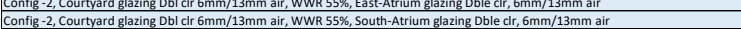 & $\frac{15.3}{14.5}$ & 6.8 & $\frac{13.7}{12.8}$ & 94.90 & $\frac{6.3}{5.0}$ & $\frac{12.7}{10.1}$ & $\frac{7.3}{7.3}$ & 6.9 & 6.99 & 90.8 & 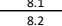 & 8.1 & \\
\hline , Courtyard glazing Doblet $6 \mathrm{~mm} / 13 \mathrm{~mm}$ air, WWR & 16.0 & 7.2 & 14.4 & 94.4 & 6.1 & 12.3 & 9.0 & 6.2 & & 89.6 & 7.9 & 7.9 & \\
\hline , Courtyard glazing D Db cr $6 \mathrm{~mm} / 13 \mathrm{~mm}$ air, WWW & 16.4 & & $\begin{array}{ll}14.8 \\
\end{array}$ & 94.9 & 5.9 & & & 6.7 & & & 3.8 & & \\
\hline 6, East-Atrium glazing Db: & $\frac{14.3}{143}$ & 6.3 & 12.6 & 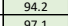 & 6.2 & 12.5 & 10.4 & 5.6 & 5.6 & 71.1. & 3.9 & 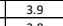 & \\
\hline (c) & $\frac{14.3}{15.0}$ & 6.3 & $\frac{12.1}{13.3}$ & 94.17 & $\frac{5.0}{6.0}$ & $\frac{9.9}{12.1}$ & 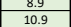 & $\frac{6.2}{5.4}$ & $\frac{6.2}{5.4}$ & & & & \\
\hline 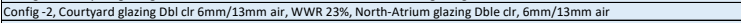 & 14.6 & 6.5 & & 94.7. & 6.0 & 12.0 & 6.9 & & & & & & \\
\hline 2, Courtyard glazing Dbl cr $6 \mathrm{~mm} / 13 \mathrm{~mm}$ air, WWR 23 & $\begin{array}{ll}13.1 \\
\text { I }\end{array}$ & 5.7 & 11.4 & 94.1 & 6.3 & 12.6 & 6.1 & 7.5 & 7.5 & 59.2 & 1.3 & 1.3 & 32.7 \\
\hline 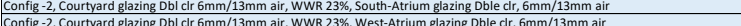 & $\frac{13.6}{138}$ & 6.0 & 11.9 & 96.9 & 5.0 & $\frac{10.1}{122}$ & $\frac{6.7}{81}$ & $\frac{7.2}{66}$ & 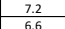 & 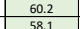 & $\frac{1.5}{10}$ & $\frac{1.5}{10}$ & $\frac{30.7}{320}$ \\
\hline 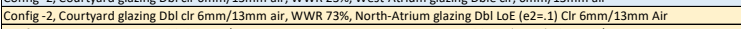 & 21.4 & 10.0 & 20.0 & 93.6 & 6.5 & $\frac{12.2}{13.0}$ & $\frac{8.1}{7.3}$ & 6.9 & 6.9 & 92.1 & 8.4 & $\frac{1.0}{8.4}$ & $\begin{array}{ll}3.0 \\
48.4\end{array}$ \\
\hline 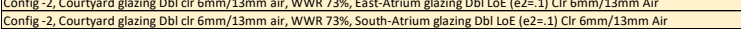 & $\frac{18.0}{16.6}$ & $\frac{8.3}{7.5}$ & $\frac{11.5}{15.1}$ & 92.9 & 6.8 & $\frac{13.7}{11.0}$ & 9.0 & $\frac{6.7}{6.2}$ & & 90.: & & & \\
\hline 2, courtyard 8 & 18. & 8.6 & $\begin{array}{ll}17.3 \\
\end{array}$ & $\begin{array}{l}93.3 \\
\end{array}$ & 6.6 & $\begin{array}{l}13.3 \\
\end{array}$ & 9.7 & 5.9 & & & 7.8 & & \\
\hline 2, Courtyard glazin & 19.4. & 9.0 & & 93.5 & 6.6 & & 10.0 & 5.7 & & 88. 5.5 & & 76 & \\
\hline Atrium glazing & $\frac{16.4}{15.5}$ & 7.4 & $\frac{14.8}{139}$ & 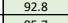 & 6.9 & 13.8 & 8.9 & 6.2 & 6 & 89 & 7.8 & 7.8 & \\
\hline 6m/m/3n & & $\frac{7.0}{7.8}$ & $\frac{13 .}{15 .}$ & 95.72 & $\frac{5.6}{6.7}$ & $\frac{1.2}{13.4}$ & & $\frac{6.1}{6.1}$ & & & $\frac{7.8}{7.7}$ & & \\
\hline & & 7.4 & 14.8 & 94.9 & 5.9 & 11.9 & 10.7 & 5.4 & & & & & \\
\hline Config-2, Courtyard glazing D DE & 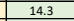 & 6.3 & 12.6 & 94.2 & 6.2 & 12.5 & 12.2 & 4.8 & & 6.9. 3 & 3.6 & 3.6 & 33.4 \\
\hline onfif-2.20urthard glazing & 14.3 & 6.3 & $\frac{12.7}{1.32} \mathrm{~T}$ & 97.1. & 5.0 & 9.99 & 10.3 & 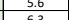 & & & 3.7 $3.7 \mathrm{C}$ & 3.7. & 31.9 \\
\hline & & & & & & & & & & & & & \\
\hline Courtyard glazing Doblct $6 \mathrm{~mm} / 13 \mathrm{~mm}$ air, WWR & 14.3 & 6.3 & $\frac{12.6}{12}$ & 92.8 & 6.9 & 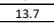 & 9.0 & 6.2 & 6.2 & 59.3 & $\begin{array}{l}1.3 \\
1.3\end{array}$ & 13 & 33.8 \\
\hline , Court & & 6.5 & & 95.7 & 5.6 & 11.2. & 9.3 & 6.0 & & & & & \\
\hline $\begin{array}{l}\text { Confifi- }- \text {, Courty } \\
\text { Config } 1 \text {, Courty }\end{array}$ & $\frac{15.0}{14.4}$ & $\frac{6.7}{6.4}$ & $\frac{13.3}{12.7}$ & $\begin{array}{l}93.3 \\
95.1\end{array}$ & $\frac{6.7}{5.9}$ & $\frac{13.3}{11.7}$ & $\frac{9.0}{1.1}$ & 6.2 & 6.7. & $\frac{58 .}{98}$ & 1.2 & $\frac{1.2}{9.8}$ & $\begin{array}{l}34.1 \\
43.9\end{array}$ \\
\hline & 9.5 & 3.8 & 7.7 & 94.5 & 6.1 & 12.2 & 0.9 & 9.7 & & & 9.9 & 9.9 & 39.5 \\
\hline $\begin{array}{l}\text { nfig- }-1, \text { Courn } \\
\text { fig, }\end{array}$ & 6.5 & 2.2 & 4.5 & 96.9 & 5.0 & 10.1 & 0.6 & 9.9 & & & 10.0 & 10.0 & 34.4 \\
\hline , ou & $\frac{10.4}{12.5}$ & $\frac{4.3}{5.4}$ & 8.6 & $\frac{94.9}{953}$ & $\frac{6.0}{58}$ & $\frac{11.9}{11.5}$ & 1.3 & $\frac{9.7}{9.6}$ & $\begin{array}{l}3.1 .6 \\
9.6\end{array}$ & 99. & $\frac{9.9}{9.8}>0$ & 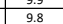 & $\begin{array}{l}4.1 .1 \\
41.6 \\
4\end{array}$ \\
\hline onfig-1, Courtyard glazi & $\frac{7.4}{8.4}$ & 3.2 & $\frac{10.8}{6.5}$ & $\begin{array}{l}95.3 . \\
944.7 \\
9\end{array}$ & 6.8 & 112.0 & 1.3 & $\begin{array}{l}3.0 \\
9.3 \\
9\end{array}$ & 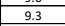 & & 9.6 & 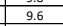 & $\frac{4.0}{37.4}$ \\
\hline 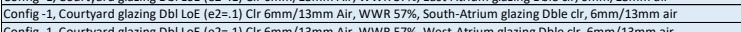 & 6.3 & 2.1 & 4.3 & 97.1 & 5.0 & 9.9 & 1.2 & 9.6 & & & 9.8 & 9.8 & 33.6 \\
\hline 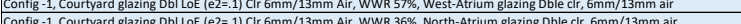 & $\frac{9.2}{13} \mathrm{~T}$ & $\frac{3.7}{5.9}+2$. & $\frac{7.3}{1.8}$ & 年.1 & 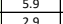 & 1.7.7 & 1.4 & $\frac{9.5}{71}$ & & & & 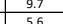 & 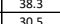 \\
\hline 1) $\operatorname{Cl~} 6 \mathrm{~mm} / 13 \mathrm{n}$ & $\begin{array}{l}13.4 \\
\end{array}$ & 5.8 & 11.6 & 101.3 & 3.1 & 6.2 & 7.6 & 6.8 & 6. & 78. & 5.4 & 5.4. & \\
\hline 1, Court & & $34,7>3$ & 6.9 & $103: \mathrm{Cl}^{-} \mathrm{C}$ & 21 & 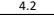 & & & & & & & \\
\hline 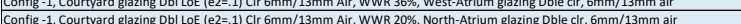 & $\frac{9.0}{9.4}$ & $\frac{3.6}{3.8}$ & $\frac{7.1}{7.6}$ & 10.6 & $\frac{2.9}{5.6}$ & $\frac{5.9}{11.2}$ & $\frac{7.2}{7.1}$ & $\frac{7.0}{7.0}$ & $\frac{7.0}{70}$ & $\frac{19.1}{66.0}$ & $\frac{5.6}{2.7}$ & $\frac{5.6}{27}$ & 2.56 28.6 \\
\hline 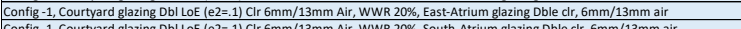 & 7.6 & 2.8 & 5.6 & 95.1 & 5.8 & 11.7 & 7.4 & 6.9 & 6.9 & 再 & 2.8 & 2.8 & 27.0 \\
\hline 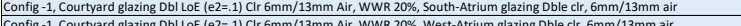 & 8.0 & $\frac{3.0}{3.2}+20$ & 6.0 & 97.4 & 4.8 & 9.7 & 8.6 & 6.3 & & & & 2.77 & 24.8 \\
\hline $\begin{array}{lll}1, \text { Coutrtyard laz } \\
1, \text { Courtyard gaz }\end{array}$ & $\frac{8.3}{16.2}$ & $\frac{3.2}{7.3}$ & $\frac{0.3}{14.6}$ & 93.1 & $\frac{3.1}{6.7}$ & $\frac{11.4}{13.5}$ & $\frac{1.6}{1.6}$ & $\frac{9.4}{9.4}$ & & & $\frac{2.7}{9.7}$ & $\frac{2.1}{9.7}$ & $\frac{27.2}{47.2}$ \\
\hline 1, , court & 11. & 4.8 & & 92.6 & 7.0 & & & 9.3 & & & 9.7 & 7 & 42.5 \\
\hline ( & 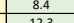 & 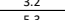 & ${ }^{6}$ & 95.6 & 5.9 & $\frac{11.8}{1.8}$ & & 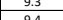 & & & 9.6 & 9.6 & 1.2. \\
\hline & 1. & 6. & $\frac{12}{12} \mathrm{C}+\mathrm{C}$ & 第3.3. & 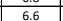 & 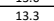 & 2.8 & 9.10 & & & 9.6 & 哥. & \\
\hline 85.5 & 10. & 4.2 & 8. & 92.8 & 6.9 & 13.8 & 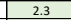 & 9.1 & & & 9.6 & 9.6 & 0.9 \\
\hline-1, Cour: & 8.25 & 3.1. & & & 5.8 & & & & & & & 9.4 & \\
\hline 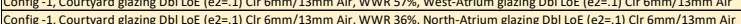 & $\frac{1.1 .1}{1.2}$ & $\frac{2.6}{6.8}$ & 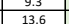 & $\begin{array}{l}\frac{93.1}{99.7} \\
9\end{array}$ & $\frac{6.7}{38}$ & $\frac{13.5}{7.6}$ & $\frac{24}{7.2}$ & $\frac{9.1}{7.0}$ & $\frac{9.1}{70}$ & $\begin{array}{l}97.2 \\
780\end{array}$ & $\frac{9.5}{5.4}$ & $\frac{9.5}{5.4}$ & 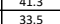 \\
\hline onfig -1, Courtyard glazin & 15.4. & 6.7 & & 99.3 & 4.0 & 7.9 & 6.1 & 7.4 & 7.4 & & 5.4 & 5.4. & 34.1 \\
\hline $\mathrm{OE}(\mathrm{e} 2=1$, $) \mathrm{Cl} 6 \mathrm{~m}$ & & 4.3 & 8.7 & 101.5 & 3.0 & 6.0 & 9.0 & 6.2 & & & & 5.3 & 26.1 \\
\hline 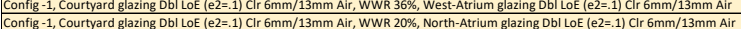 & $\frac{10.7}{11.3}$ & 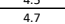 & 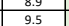 & 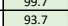 & $\frac{3.8}{6.5}$ & $\frac{1}{13}$ & $\frac{6.6}{6.4}$ & $\frac{1.3}{7.3}$ & $\frac{1.3}{7.3}$ & $\frac{70.4}{663}$ & $\frac{3.8}{2.8}$ & 2.8 & $\frac{29.2}{32.6}$ \\
\hline ing Dol LoE (e2=:1) Cr $6 \mathrm{~mm} / 13 \mathrm{~mm}$ Air & 9.4 & 3.8 & 7.6 ( & 93.2 & 6.7 & 13.4 & 8.0 & 6.6 & & 66. & 2.7 & 2.7 & 30.3 \\
\hline 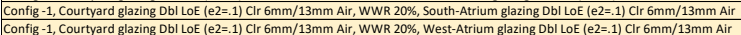 & $\frac{9.8}{10.1}$ & $\frac{4.0}{4.1}$ & $\frac{3.3}{8.3}$ & $\frac{95.5}{93.5}$ & \begin{tabular}{|c|c|cc}
5.7 \\
6.6
\end{tabular} & $\frac{x_{11.4}^{13.1}}{13 .}$ & $\begin{array}{l}4.4 \\
8.1\end{array}$ & $\begin{array}{l}8.2 \\
6.6\end{array}$ & $\begin{array}{l}8.2 \\
6.6\end{array}$ & 65.7 & $\begin{array}{l}2.8 \\
2.7\end{array}$ & $\begin{array}{l}2.8 \\
2.7\end{array}$ & 30.6 \\
\hline
\end{tabular}




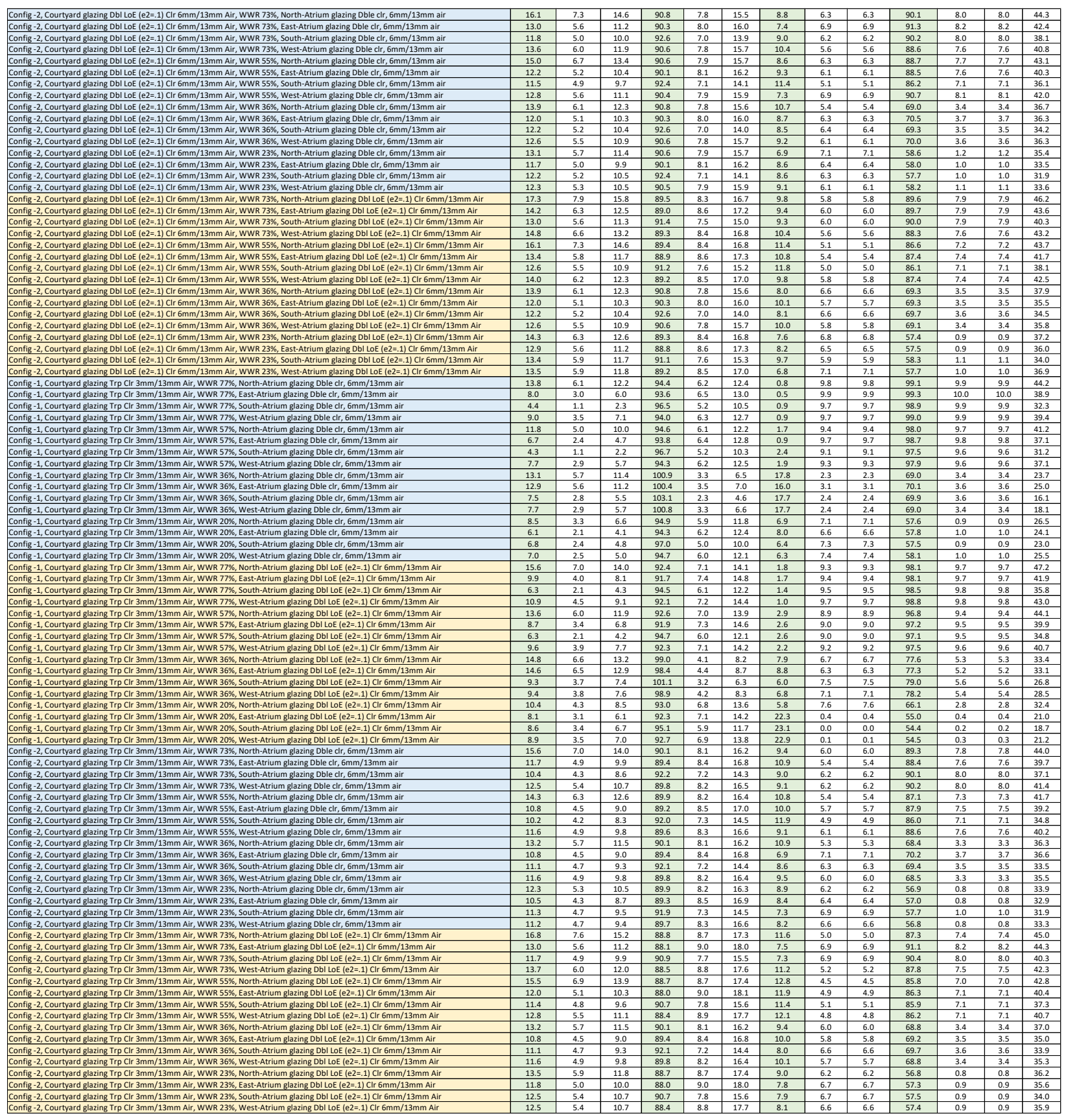




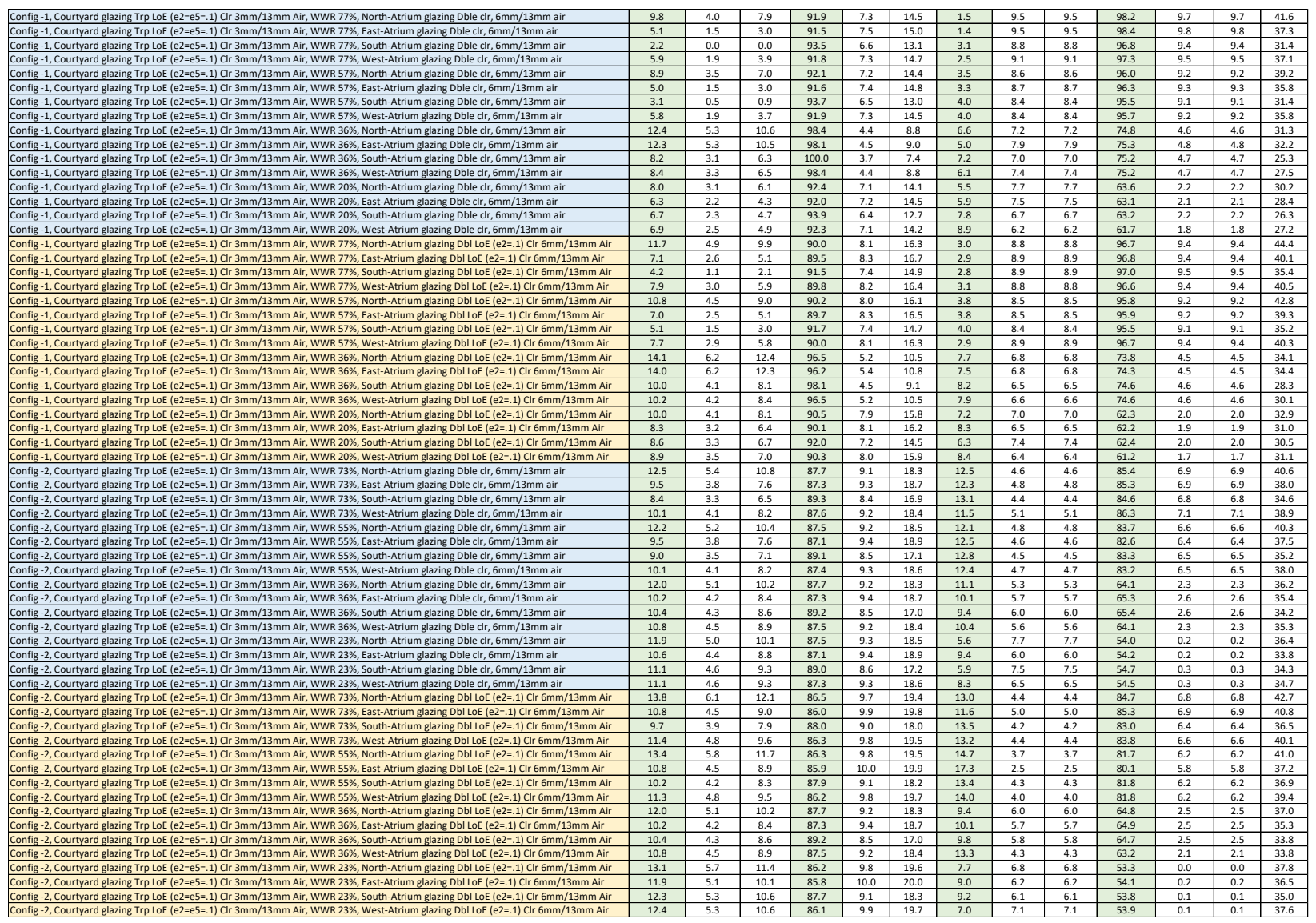




\begin{tabular}{|c|c|c|}
\hline & Column1 & Column2 -t \\
\hline SI. No & Options & Weighted total \\
\hline & Config -1 , Courtyard glazing Dbl clr $6 \mathrm{~mm} / 13 \mathrm{~mm}$ air, WWR $77 \%$, North-Atrium glazing DbI LoE (e2=.1) Clr $6 \mathrm{~mm} / 13 \mathrm{~mm}$ & 49 \\
\hline 2 & Config -2 , Courtyard glazing Dbl clr $6 \mathrm{~mm} / 13 \mathrm{~mm}$ air, WWR $73 \%$, North-Atrium glazing Dbl LoE (e2=.1) Clr $6 \mathrm{~mm} / 13 \mathrm{~mm}$ & 48 \\
\hline 3 & Config -1 , Courtyard glazing DbI LoE (e2=.1) Clr $6 \mathrm{~mm} / 13 \mathrm{~mm}$ Air, WWR 77\%, North-Atrium glazing DbI LoE (e2=.1) Clr & 47 \\
\hline 4 & Config -1, Courtyard glazing Trp Clr 3mm/13mm Air, WWR 77\%, North-Atrium glazing Dbl LoE (e2=.1) Clr 6mm/13mr & 47 \\
\hline & Config -2 , Courtyard glazing DbI LoE (e2=.1) Clr $6 \mathrm{~mm} / 13 \mathrm{~mm}$ Air, WWR 73\%, North-Atrium glazing DbI LoE (e2=.1) Clr & 46 \\
\hline 6 & Config -2, Courtyard glazing Dbl clr $6 \mathrm{~mm} / 13 \mathrm{~mm}$ air, WWR $73 \%$, North-Atrium glazing Dble $\mathrm{clr}, 6 \mathrm{~mm} / 13 \mathrm{~mm}$ air & 46 \\
\hline 7 & Config -1 , Courtyard glazing Dbl clr $6 \mathrm{~mm} / 13 \mathrm{~mm}$ air, WWR $77 \%$, North-Atrium glazing Dble clr, $6 \mathrm{~mm} / 13 \mathrm{~mm}$ air & 46 \\
\hline 8 & Config -1, Courtyard glazing Dbl clr $6 \mathrm{~mm} / 13 \mathrm{~mm}$ air, WWR 57\%, North-Atrium glazing DbI LoE (e2=.1) Clr $6 \mathrm{~mm} / 13 \mathrm{~mm}$ & 45 \\
\hline 9 & Config -2 , Courtyard glazing Dbl clr $6 \mathrm{~mm} / 13 \mathrm{~mm}$ air, WWR $73 \%$, East-Atrium glazing Dbl LoE (e2=.1) Clr $6 \mathrm{~mm} / 13 \mathrm{~mm}$ & 45 \\
\hline 10 & Config -1 , Courtyard glazing Dbl clr $6 \mathrm{~mm} / 13 \mathrm{~mm}$ air, WWR $77 \%$, West-Atrium glazing Dbl LoE (e2=.1) Clr $6 \mathrm{~mm} / 13 \mathrm{~mm}$ & 45 \\
\hline 11 & Config -2 , Courtyard glazing Trp Clr 3mm/13mm Air, WWR 73\%, North-Atrium glazing Dbl LoE (e2=.1) Clr 6mm/13mn & 45 \\
\hline 12 & Config -1 , Courtyard glazing Dbl clr $6 \mathrm{~mm} / 13 \mathrm{~mm}$ air, WWR $77 \%$, East-Atrium glazing Dbl LoE (e2=.1) Clr $6 \mathrm{~mm} / 13 \mathrm{~mm} /$ & 45 \\
\hline 13 & Config -1 , Courtyard glazing DbI LoE (e2=.1) Clr $6 \mathrm{~mm} / 13 \mathrm{~mm}$ Air, WWR $57 \%$, North-Atrium glazing DbI LoE (e2=.1) Clr & 45 \\
\hline 14 & Config -2 , Courtyard glazing Dbl clr $6 \mathrm{~mm} / 13 \mathrm{~mm}$ air, WWR 55\%, North-Atrium glazing Dbl LoE (e2=.1) Clr $6 \mathrm{~mm} / 13 \mathrm{~mm}$ & 45 \\
\hline 15 & Config -1 , Courtyard glazing Trp LoE (e2=e5=.1) Clr 3mm/13mm Air, WWR 77\%, North-Atrium glazing Dbl LoE (e2=.1) & 44 \\
\hline 16 & Config -2 , Courtyard glazing DbI LoE (e2=.1) Clr $6 \mathrm{~mm} / 13 \mathrm{~mm}$ Air, WWR 73\%, North-Atrium glazing Dble clr, $6 \mathrm{~mm} / 13 \mathrm{~m}$ & 44 \\
\hline 17 & Config -2, Courtyard glazing Trp Clr 3mm/13mm Air, WWR 73\%, East-Atrium glazing Dbl LoE (e2=.1) Clr $6 \mathrm{~mm} / 13 \mathrm{~mm}$ & 44 \\
\hline 18 & Config -2, Courtyard glazing Dbl clr $6 \mathrm{~mm} / 13 \mathrm{~mm}$ air, WWR 73\%, West-Atrium glazing Dbl LoE (e2=.1) Clr $6 \mathrm{~mm} / 13 \mathrm{~mm}$ & 44 \\
\hline 19 & Config -1, Courtyard glazing Trp Clr 3mm/13mm Air, WWR 77\%, North-Atrium glazing Dble clr, $6 \mathrm{~mm} / 13 \mathrm{~mm}$ air & 44 \\
\hline 20 & Config -1, Courtyard glazing Trp Clr 3mm/13mm Air, WWR 57\%, North-Atrium glazing Dbl LoE (e2=.1) Clr 6mm/13mr & 44 \\
\hline 21 & Config -2 , Courtyard glazing Dbl clr $6 \mathrm{~mm} / 13 \mathrm{~mm}$ air, WWR 55\%, North-Atrium glazing Dble clr, $6 \mathrm{~mm} / 13 \mathrm{~mm}$ air & 44 \\
\hline 22 & Config -2, Courtyard glazing Trp Clr 3mm/13mm Air, WWR 73\%, North-Atrium glazing Dble clr, $6 \mathrm{~mm} / 13 \mathrm{~mm}$ air & 44 \\
\hline 23 & Config -1 , Courtyard glazing DbI LoE (e2=.1) Clr $6 \mathrm{~mm} / 13 \mathrm{~mm}$ Air, WWR $77 \%$, North-Atrium glazing Dble clr, $6 \mathrm{~mm} / 13 \mathrm{~m}$ & 44 \\
\hline 24 & Config -2 , Courtyard glazing Dbl clr $6 \mathrm{~mm} / 13 \mathrm{~mm}$ air, WWR $73 \%$, East-Atrium glazing Dble clr, $6 \mathrm{~mm} / 13 \mathrm{~mm}$ air & 44 \\
\hline 25 & Config -2 , Courtyard glazing DbI LoE (e2=.1) Clr $6 \mathrm{~mm} / 13 \mathrm{~mm}$ Air, WWR 55\%, North-Atrium glazing Dbl LoE (e2=.1) Clr & 44 \\
\hline 26 & Config -2, Courtyard glazing DbI LoE (e2=.1) Clr $6 \mathrm{~mm} / 13 \mathrm{~mm}$ Air, WWR 73\%, East-Atrium glazing DbI LoE (e2=.1) Clr 6 & 44 \\
\hline 27 & Config -2 , Courtyard glazing Dbl clr $6 \mathrm{~mm} / 13 \mathrm{~mm}$ air, WWR $73 \%$, West-Atrium glazing Dble clr, $6 \mathrm{~mm} / 13 \mathrm{~mm}$ air & 43 \\
\hline 28 & Config -1, Courtyard glazing Dbl LoE (e2=.1) Clr $6 \mathrm{~mm} / 13 \mathrm{~mm}$ Air, WWR 77\%, West-Atrium glazing DbI LoE (e2=.1) Clr & 43 \\
\hline 29 & Config -2 , Courtyard glazing DbI LoE (e2=.1) Clr $6 \mathrm{~mm} / 13 \mathrm{~mm}$ Air, WWR 73\%, West-Atrium glazing DbI LoE (e2=.1) Clr & 43 \\
\hline 30 & Config -2, Courtyard glazing DbI LoE (e2=.1) Clr $6 \mathrm{~mm} / 13 \mathrm{~mm}$ Air, WWR 55\%, North-Atrium glazing Dble clr, $6 \mathrm{~mm} / 13 \mathrm{~m}$ & 43 \\
\hline 31 & Config -1 , Courtyard glazing Trp Clr 3mm/13mm Air, WWR 77\%, West-Atrium glazing DbI LoE (e2=.1) Clr $6 \mathrm{~mm} / 13 \mathrm{~mm}$ & 43 \\
\hline 32 & Config -2 , Courtyard glazing Trp Clr 3mm/13mm Air, WWR 55\%, North-Atrium glazing Dbl LoE (e2=.1) Clr $6 \mathrm{~mm} / 13 \mathrm{mr}$ & 43 \\
\hline 33 & Config -2, Courtyard glazing Dbl clr $6 \mathrm{~mm} / 13 \mathrm{~mm}$ air, WWR 55\%, West-Atrium glazing DbI LoE (e2=.1) Clr $6 \mathrm{~mm} / 13 \mathrm{~mm}$ & 43 \\
\hline 34 & Config -1 , Courtyard glazing Trp LoE (e2=e5=.1) Clr 3mm/13mm Air, WWR 57\%, North-Atrium glazing DbI LoE (e2=.1) & 43 \\
\hline 35 & Config -2 , Courtyard glazing Trp LoE (e2=e5=.1) Clr 3mm/13mm Air, WWR 73\%, North-Atrium glazing DbI LoE (e2=.1) & 43 \\
\hline 36 & Config -2, Courtyard glazing Dbl clr $6 \mathrm{~mm} / 13 \mathrm{~mm}$ air, WWR 55\%, East-Atrium glazing Dbl LoE (e2=.1) Clr $6 \mathrm{~mm} / 13 \mathrm{~mm}$ & 43 \\
\hline 37 & Config -1, Courtyard glazing DbI LoE (e2=.1) Clr 6mm/13mm Air, WWR 77\%, East-Atrium glazing DbI LoE (e2=.1) Clr 6 & 43 \\
\hline 38 & Config -2, Courtyard glazing Dbl LoE (e2=.1) Clr $6 \mathrm{~mm} / 13 \mathrm{~mm}$ Air, WWR 55\%, West-Atrium glazing DbI LoE (e2=.1) Clr & 43 \\
\hline 39 & Config -1, Courtyard glazing Dbl clr 6mm/13mm air, WWR 57\%, North-Atrium glazing Dble clr, $6 \mathrm{~mm} / 13 \mathrm{~mm}$ air & 42 \\
\hline 40 & Config -2 , Courtyard glazing Dbl LoE (e2=.1) Clr $6 \mathrm{~mm} / 13 \mathrm{~mm}$ Air, WWR 73\%, East-Atrium glazing Dble clr, $6 \mathrm{~mm} / 13 \mathrm{~mm}$ & 42 \\
\hline 41 & Config -2 , Courtyard glazing Trp Clr 3mm/13mm Air, WWR 73\%, West-Atrium glazing Dbl LoE (e2=.1) Clr $6 \mathrm{~mm} / 13 \mathrm{~mm}$ & 42 \\
\hline 42 & Config -1, Courtyard glazing Dbl clr $6 \mathrm{~mm} / 13 \mathrm{~mm}$ air, WWR 57\%, West-Atrium glazing Dbl LoE (e2=.1) Clr $6 \mathrm{~mm} / 13 \mathrm{~mm}$ & 42 \\
\hline 43 & Config -2 , Courtyard glazing DbI LoE (e2=.1) Clr $6 \mathrm{~mm} / 13 \mathrm{~mm}$ Air, WWR 55\%, West-Atrium glazing Dble clr, $6 \mathrm{~mm} / 13 \mathrm{~m}$ & 42 \\
\hline 44 & Config -1, Courtyard glazing Trp Clr 3mm/13mm Air, WWR 77\%, East-Atrium glazing Dbl LoE (e2=.1) Clr 6mm/13mm & 42 \\
\hline 45 & Config -1 , Courtyard glazing Dbl clr $6 \mathrm{~mm} / 13 \mathrm{~mm}$ air, WWR $57 \%$, East-Atrium glazing Dbl LoE (e2=.1) Clr $6 \mathrm{~mm} / 13 \mathrm{~mm} /$ & 42 \\
\hline 46 & Config - 1 , Courtyard glazing Dbl clr $6 \mathrm{~mm} / 13 \mathrm{~mm}$ air, WWR $77 \%$, West-Atrium glazing Dble clr, $6 \mathrm{~mm} / 13 \mathrm{~mm}$ air & 42 \\
\hline 47 & Config -2, Courtyard glazing Trp Clr 3mm/13mm Air, WWR 55\%, North-Atrium glazing Dble clr, $6 \mathrm{~mm} / 13 \mathrm{~mm}$ air & 42 \\
\hline 48 & Config -2 , Courtyard glazing DbI LoE (e2=.1) Clr $6 \mathrm{~mm} / 13 \mathrm{~mm}$ Air, WWR 55\%, East-Atrium glazing DbI LoE (e2=.1) Clr 6 & 42 \\
\hline 49 & Config -1, Courtyard glazing Dbl LoE (e2=.1) Clr $6 \mathrm{~mm} / 13 \mathrm{~mm}$ Air, WWR 57\%, North-Atrium glazing Dble clr, $6 \mathrm{~mm} / 13 \mathrm{~m}$ & 42 \\
\hline 50 & Config -1 , Courtyard glazing Trp LoE (e2=e5=.1) Clr 3mm/13mm Air, WWR 77\%, North-Atrium glazing Dble clr, $6 \mathrm{~mm} /$ & 42 \\
\hline 51 & Config -1 , Courtyard glazing Dbl clr $6 \mathrm{~mm} / 13 \mathrm{~mm}$ air, WWR $77 \%$, East-Atrium glazing Dble clr, $6 \mathrm{~mm} / 13 \mathrm{~mm}$ air & 41 \\
\hline 52 & Config -2, Courtyard glazing Dbl clr $6 \mathrm{~mm} / 13 \mathrm{~mm}$ air, WWR 55\%, East-Atrium glazing Dble clr, $6 \mathrm{~mm} / 13 \mathrm{~mm}$ air & 41 \\
\hline 53 & Config -2, Courtyard glazing Trp Clr 3mm/13mm Air, WWR 73\%, West-Atrium glazing Dble clr, $6 \mathrm{~mm} / 13 \mathrm{~mm}$ air & 41 \\
\hline 54 & Config -1 , Courtyard glazing DbI LoE (e2=.1) Clr $6 \mathrm{~mm} / 13 \mathrm{~mm}$ Air, WWR 57\%, West-Atrium glazing DbI LoE (e2=.1) Clr & 41 \\
\hline 55 & Config -1, Courtyard glazing Trp Clr 3mm/13mm Air, WWR 57\%, North-Atrium glazing Dble clr, $6 \mathrm{~mm} / 13 \mathrm{~mm}$ air & 41 \\
\hline 56 & Config -2 , Courtyard glazing Trp LoE (e2=e5=.1) Clr 3mm/13mm Air, WWR 55\%, North-Atrium glazing DbI LoE (e2=.1) & 41 \\
\hline 57 & Config -1, Courtyard glazing DbI LoE (e2=.1) Clr $6 \mathrm{~mm} / 13 \mathrm{~mm}$ Air, WWR 57\%, East-Atrium glazing DbI LoE (e2=.1) Clr 6 & 41 \\
\hline 58 & Config -2 , Courtyard glazing Dbl LoE $(\mathrm{e} 2=.1) \mathrm{Clr} 6 \mathrm{~mm} / 13 \mathrm{~mm}$ Air, WWR $73 \%$, West-Atrium glazing Dble clr, $6 \mathrm{~mm} / 13 \mathrm{~m}$ & 41 \\
\hline 59 & Config -2 , Courtyard glazing Trp LoE (e2=e5=.1) Clr 3mm/13mm Air, WWR 73\%, East-Atrium glazing DbI LoE (e2=.1) $\mathrm{C}$ & 41 \\
\hline 60 & Config -2, Courtyard glazing Dbl clr 6mm/13mm air, WWR 55\%, West-Atrium glazing Dble clr, $6 \mathrm{~mm} / 13 \mathrm{~mm}$ air & 41 \\
\hline 61 & Config -2 , Courtyard glazing Trp Clr 3mm/13mm Air, WWR 55\%, West-Atrium glazing DbI LoE (e2=.1) Clr $6 \mathrm{~mm} / 13 \mathrm{~mm}$ & 41 \\
\hline 62 & Config -1 , Courtyard glazing Trp Clr 3mm/13mm Air, WWR 57\%, West-Atrium glazing Dbl LoE (e2=.1) Clr $6 \mathrm{~mm} / 13 \mathrm{~mm}$ & 41 \\
\hline 63 & Config -2 , Courtyard glazing Trp LoE (e2=e5=.1) Clr 3mm/13mm Air, WWR 73\%, North-Atrium glazing Dble clr, $6 \mathrm{~mm} /$ & 41 \\
\hline 64 & Config -1 , Courtyard glazing Trp LoE (e2=e5=.1) Clr 3mm/13mm Air, WWR 77\%, West-Atrium glazing DbI LoE (e2=.1) & 41 \\
\hline 65 & Config -2 , Courtyard glazing Trp Clr 3mm/13mm Air, WWR 55\%, East-Atrium glazing Dbl LoE (e2=.1) Clr 6mm/13mm & 40 \\
\hline 66 & Config -2, Courtyard glazing Dbl clr $6 \mathrm{~mm} / 13 \mathrm{~mm}$ air, WWR $73 \%$, South-Atrium glazing Dbl LoE (e2=.1) Clr $6 \mathrm{~mm} / 13 \mathrm{~mm}$ & 40 \\
\hline 67 & Config -1 , Courtyard glazing Trp LoE (e2=e5=.1) Clr 3mm/13mm Air, WWR 57\%, West-Atrium glazing DbI LoE (e2=.1) & 40 \\
\hline 68 & Config -2, Courtyard glazing Trp Clr 3mm/13mm Air, WWR 73\%, South-Atrium glazing DbI LoE (e2=.1) Clr $6 \mathrm{~mm} / 13 \mathrm{mr}$ & 40 \\
\hline 69 & Config -2, Courtyard glazing DbI LoE (e2=.1) Clr $6 \mathrm{~mm} / 13 \mathrm{~mm}$ Air, WWR 55\%, East-Atrium glazing Dble clr, $6 \mathrm{~mm} / 13 \mathrm{~mm}$ & 40 \\
\hline 70 & Config -2, Courtyard glazing DbI LoE (e2=.1) Clr $6 \mathrm{~mm} / 13 \mathrm{~mm}$ Air, WWR 73\%, South-Atrium glazing DbI LoE (e2=.1) Clr & 40 \\
\hline
\end{tabular}




\begin{tabular}{|c|c|c|}
\hline 71 & Config -2 , Courtyard glazing Trp LoE (e2=e5=.1) Clr 3mm/13mm Air, WWR 55\%, North-Atrium glazing Dble clr, $6 \mathrm{~mm} /$ & 40 \\
\hline 72 & Config -2, Courtyard glazing Trp Clr 3mm/13mm Air, WWR 55\%, West-Atrium glazing Dble clr, $6 \mathrm{~mm} / 13 \mathrm{~mm}$ air & 40 \\
\hline 73 & Config -1 , Courtyard glazing Trp LoE (e2=e5=.1) Clr 3mm/13mm Air, WWR 77\%, East-Atrium glazing DbI LoE (e2=.1) C & 40 \\
\hline 74 & Config -1 , Courtyard glazing Dbl LoE (e2=.1) Clr $6 \mathrm{~mm} / 13 \mathrm{~mm}$ Air, WWR $77 \%$, West-Atrium glazing Dble clr, $6 \mathrm{~mm} / 13 \mathrm{~m}$ & 40 \\
\hline 76 & Config -1, Courtyard glazing Trp Clr 3mm/13mm Air, WWR 57\%, East-Atrium glazing DbI LoE (e2=.1) Clr 6mm/13mm & 40 \\
\hline 77 & Config -2 , Courtyard glazing Trp Clr 3mm/13mm Air, WWR 73\%, East-Atrium glazing Dble clr, $6 \mathrm{~mm} / 13 \mathrm{~mm}$ air & 40 \\
\hline 78 & Config -1 , Courtyard glazing DbI LoE (e2=.1) Clr $6 \mathrm{~mm} / 13 \mathrm{~mm}$ Air, WWR 77\%, East-Atrium glazing Dble clr, $6 \mathrm{~mm} / 13 \mathrm{mn}$ & 39 \\
\hline 81 & Config -1 , Courtyard glazing Trp LoE (e2=e5=.1) Clr 3mm/13mm Air, WWR 57\%, East-Atrium glazing DbI LoE (e2=.1) C & 39 \\
\hline 82 & Config -2 , Courtyard glazing Trp Clr 3mm/13mm Air, WWR 55\%, East-Atrium glazing Dble clr, $6 \mathrm{~mm} / 13 \mathrm{~mm}$ air & 39 \\
\hline 83 & Config -1 , Courtyard glazing Trp LoE (e2=e5=.1) Clr 3mm/13mm Air, WWR 57\%, North-Atrium glazing Dble clr, $6 \mathrm{~mm} /$ & 39 \\
\hline 84 & Config -1 , Courtyard glazing Dbl clr $6 \mathrm{~mm} / 13 \mathrm{~mm}$ air, WWR 57\%, West-Atrium glazing Dble clr, $6 \mathrm{~mm} / 13 \mathrm{~mm}$ air & 39 \\
\hline 85 & Config -2 , Courtyard glazing Dbl clr $6 \mathrm{~mm} / 13 \mathrm{~mm}$ air, WWR 55\%, South-Atrium glazing DbI LoE (e2=.1) Clr $6 \mathrm{~mm} / 13 \mathrm{~mm}$ & 39 \\
\hline 90 & Config -1, Courtyard glazing DbI LoE (e2=.1) Clr $6 \mathrm{~mm} / 13 \mathrm{~mm}$ Air, WWR 57\%, West-Atrium glazing Dble clr, $6 \mathrm{~mm} / 13 \mathrm{~m}$ & 38 \\
\hline 91 & Config -2 , Courtyard glazing Dbl clr $6 \mathrm{~mm} / 13 \mathrm{~mm}$ air, WWR $73 \%$, South-Atrium glazing Dble clr, $6 \mathrm{~mm} / 13 \mathrm{~mm}$ air & 38 \\
\hline 92 & Config -2 , Courtyard glazing DbI LoE (e2=.1) Clr $6 \mathrm{~mm} / 13 \mathrm{~mm}$ Air, WWR 55\%, South-Atrium glazing DbI LoE (e2=.1) Clr & 38 \\
\hline 93 & Config -2 , Courtyard glazing DbI LoE (e2=.1) Clr $6 \mathrm{~mm} / 13 \mathrm{~mm}$ Air, WWR 73\%, South-Atrium glazing Dble clr, $6 \mathrm{~mm} / 13 \mathrm{~m}$ & 38 \\
\hline 94 & Config -2, Courtyard glazing Dbl clr $6 \mathrm{~mm} / 13 \mathrm{~mm}$ air, WWR 55\%, South-Atrium glazing Dble clr, $6 \mathrm{~mm} / 13 \mathrm{~mm}$ air & 38 \\
\hline 95 & Config -2, Courtyard glazing Trp LoE (e2=e5=.1) Clr 3mm/13mm Air, WWR 73\%, East-Atrium glazing Dble clr, $6 \mathrm{~mm} / 1$ : & 38 \\
\hline 96 & Config -2 , Courtyard glazing Trp LoE (e2=e5=.1) Clr 3mm/13mm Air, WWR 55\%, West-Atrium glazing Dble clr, $6 \mathrm{~mm} / 1$ & 38 \\
\hline 97 & Config -2 , Courtyard glazing DbI LoE (e2=.1) Clr $6 \mathrm{~mm} / 13 \mathrm{~mm}$ Air, WWR $36 \%$, North-Atrium glazing DbI LoE (e2=.1) Clr & 38 \\
\hline 98 & Config -2, Courtyard glazing Trp LoE (e2=e5=.1) Clr 3mm/13mm Air, WWR 23\%, North-Atrium glazing Dbl LoE (e2=.1) & 38 \\
\hline 99 & Config -2 , Courtyard glazing Trp LoE (e2=e5=.1) Clr 3mm/13mm Air, WWR 23\%, West-Atrium glazing DbI LoE (e2=.1) & 38 \\
\hline 100 & Config -2, Courtyard glazing Trp LoE (e2=e5=.1) Clr 3mm/13mm Air, WWR 55\%, East-Atrium glazing Dble clr, 6mm/1: & 38 \\
\hline 109 & Config -1, Courtyard glazing Trp Clr 3mm/13mm Air, WWR 57\%, East-Atrium glazing Dble clr, $6 \mathrm{~mm} / 13 \mathrm{~mm}$ air & 37 \\
\hline 110 & Config -1, Courtyard glazing Trp Clr 3mm/13mm Air, WWR 57\%, West-Atrium glazing Dble clr, $6 \mathrm{~mm} / 13 \mathrm{~mm}$ air & 37 \\
\hline 111 & Config -2 , Courtyard glazing Trp Clr 3mm/13mm Air, WWR 73\%, South-Atrium glazing Dble clr, $6 \mathrm{~mm} / 13 \mathrm{~mm}$ air & 37 \\
\hline 112 & Config -2 , Courtyard glazing Trp Clr 3mm/13mm Air, WWR 36\%, North-Atrium glazing DbI LoE (e2=.1) Clr 6mm/13mr & 37 \\
\hline 113 & Config -2 , Courtyard glazing Trp LoE (e2=e5=.1) Clr 3mm/13mm Air, WWR 36\%, North-Atrium glazing DbI LoE (e2=.1) & 37 \\
\hline 114 & Config -2 , Courtyard glazing Trp LoE (e2=e5=.1) Clr 3mm/13mm Air, WWR 55\%, South-Atrium glazing Dbl LoE (e2=.1) & 37 \\
\hline 115 & Config -2 , Courtyard glazing DbI LoE (e2=.1) Clr $6 \mathrm{~mm} / 13 \mathrm{~mm}$ Air, WWR 23\%, West-Atrium glazing DbI LoE (e2=.1) Clr & 37 \\
\hline 116 & Config -1 , Courtyard glazing Dbl clr $6 \mathrm{~mm} / 13 \mathrm{~mm}$ air, WWR $57 \%$, South-Atrium glazing DbI LoE (e2=.1) Clr $6 \mathrm{~mm} / 13 \mathrm{~mm}$ & 37 \\
\hline 117 & Config -2 , Courtyard glazing DbI LoE (e2=.1) Clr $6 \mathrm{~mm} / 13 \mathrm{~mm}$ Air, WWR 36\%, North-Atrium glazing Dble clr, $6 \mathrm{~mm} / 13 \mathrm{~m}$ & 37 \\
\hline 118 & Config -2 , Courtyard glazing Trp Clr 3mm/13mm Air, WWR 36\%, East-Atrium glazing Dble clr, $6 \mathrm{~mm} / 13 \mathrm{~mm}$ air & 37 \\
\hline 119 & Config -2 , Courtyard glazing Trp LoE (e2=e5=.1) Clr 3mm/13mm Air, WWR 73\%, South-Atrium glazing DbI LoE (e2=.1) & 37 \\
\hline 120 & Config -2 , Courtyard glazing Trp LoE (e2=e5=.1) Clr 3mm/13mm Air, WWR 23\%, East-Atrium glazing DbI LoE (e2=.1) $\mathrm{C}$ & 36 \\
\hline 121 & Config -2 , Courtyard glazing Trp LoE (e2=e5=.1) Clr 3mm/13mm Air, WWR 23\%, North-Atrium glazing Dble clr, $6 \mathrm{~mm} /$ & 36 \\
\hline 122 & Config -2, Courtyard glazing DbI LoE (e2=.1) Clr $6 \mathrm{~mm} / 13 \mathrm{~mm}$ Air, WWR $36 \%$, West-Atrium glazing Dble clr, $6 \mathrm{~mm} / 13 \mathrm{~m}$ & 36 \\
\hline 123 & Config -2 , Courtyard glazing DbI LoE (e2=.1) Clr $6 \mathrm{~mm} / 13 \mathrm{~mm}$ Air, WWR 36\%, East-Atrium glazing Dble clr, $6 \mathrm{~mm} / 13 \mathrm{mn}$ & 36 \\
\hline 124 & Config -2, Courtyard glazing Trp Clr 3mm/13mm Air, WWR 36\%, North-Atrium glazing Dble clr, $6 \mathrm{~mm} / 13 \mathrm{~mm}$ air & 36 \\
\hline 125 & Config -1 , Courtyard glazing DbI LoE (e2=.1) Clr 6mm/13mm Air, WWR 57\%, South-Atrium glazing DbI LoE (e2=.1) Clr & 36 \\
\hline 126 & Config -2 , Courtyard glazing Trp LoE (e2=e5=.1) Clr 3mm/13mm Air, WWR 36\%, North-Atrium glazing Dble clr, $6 \mathrm{~mm} /$ & 36 \\
\hline 127 & Config -2 , Courtyard glazing Trp Clr 3mm/13mm Air, WWR 23\%, North-Atrium glazing Dbl LoE (e2=.1) Clr $6 \mathrm{~mm} / 13 \mathrm{mr}$ & 36 \\
\hline 128 & Config -2 , Courtyard glazing DbI LoE (e2=.1) Clr $6 \mathrm{~mm} / 13 \mathrm{~mm}$ Air, WWR 55\%, South-Atrium glazing Dble clr, $6 \mathrm{~mm} / 13 \mathrm{~m}$ & 36 \\
\hline 129 & Config -2 , Courtyard glazing DbI LoE (e2=.1) Clr $6 \mathrm{~mm} / 13 \mathrm{~mm}$ Air, WWR 23\%, East-Atrium glazing DbI LoE (e2=.1) Clr 6 & 36 \\
\hline 130 & Config -2, Courtyard glazing Trp Clr 3mm/13mm Air, WWR 23\%, West-Atrium glazing DbI LoE (e2=.1) Clr 6mm/13mm & 36 \\
\hline 131 & Config -2 , Courtyard glazing Dbl clr $6 \mathrm{~mm} / 13 \mathrm{~mm}$ air, WWR $36 \%$, North-Atrium glazing Dbl LoE (e2=.1) Clr $6 \mathrm{~mm} / 13 \mathrm{~mm}$ & 36 \\
\hline
\end{tabular}


141 Config -2, Courtyard glazing Trp LoE (e2=e5=.1) Clr 3mm/13mm Air, WWR 36\%, East-Atrium glazing Dble clr, 6mm/1:

142 Config -2, Courtyard glazing Dbl LoE (e2=.1) Clr 6mm/13mm Air, WWR 23\%, North-Atrium glazing Dble clr, 6mm/13m

143 Config -2, Courtyard glazing Trp LoE (e2=e5=.1) Clr 3mm/13mm Air, WWR 36\%, East-Atrium glazing Dbl LoE (e2=.1) C

144 Config -2, Courtyard glazing Trp Clr 3mm/13mm Air, WWR 36\%, West-Atrium glazing Dbl LoE (e2=.1) Clr $6 \mathrm{~mm} / 13 \mathrm{~mm}$

145 Config -2, Courtyard glazing Trp LoE (e2=e5=.1) Clr 3mm/13mm Air, WWR 36\%, West-Atrium glazing Dble clr, $6 \mathrm{~mm} / 1$

146 Config -1, Courtyard glazing Trp LoE (e2=e5=.1) Clr 3mm/13mm Air, WWR 57\%, South-Atrium glazing Dbl LoE (e2=.1)

147 Config -2 , Courtyard glazing Trp LoE (e2=e5=.1) Clr 3mm/13mm Air, WWR 55\%, South-Atrium glazing Dble clr, 6mm/

148 Config -2, Courtyard glazing Trp LoE (e2=e5=.1) Clr 3mm/13mm Air, WWR 23\%, South-Atrium glazing DbI LoE (e2=.1)

149 Config -2, Courtyard glazing Trp Clr 3mm/13mm Air, WWR 36\%, East-Atrium glazing Dbl LoE (e2=.1) Clr 6mm/13mm

150 Config -1, Courtyard glazing Dbl clr $6 \mathrm{~mm} / 13 \mathrm{~mm}$ air, WWR 77\%, South-Atrium glazing Dble clr, $6 \mathrm{~mm} / 13 \mathrm{~mm}$ air

151 Config -2, Courtyard glazing Trp Clr 3mm/13mm Air, WWR 55\%, South-Atrium glazing Dble clr, $6 \mathrm{~mm} / 13 \mathrm{~mm}$ air

152 Config -1, Courtyard glazing Trp Clr 3mm/13mm Air, WWR 57\%, South-Atrium glazing DbI LoE (e2=.1) Clr 6mm/13mr

153 Config -2, Courtyard glazing Trp LoE (e2=e5=.1) Clr 3mm/13mm Air, WWR 23\%, West-Atrium glazing Dble clr, 6mm/1

154 Config -2, Courtyard glazing Trp LoE (e2=e5=.1) Clr 3mm/13mm Air, WWR 73\%, South-Atrium glazing Dble clr, 6mm/

155 Config -2, Courtyard glazing Dbl LoE (e2=.1) Clr 6mm/13mm Air, WWR 36\%, South-Atrium glazing Dbl LoE (e2=.1) Clr

156 Config -2, Courtyard glazing Dbl clr $6 \mathrm{~mm} / 13 \mathrm{~mm}$ air, WWR $36 \%$, East-Atrium glazing Dble clr, $6 \mathrm{~mm} / 13 \mathrm{~mm}$ air

157 Config -2, Courtyard glazing Dbl clr $6 \mathrm{~mm} / 13 \mathrm{~mm}$ air, WWR 23\%, North-Atrium glazing Dbl LoE (e2=.1) Clr $6 \mathrm{~mm} / 13 \mathrm{~mm}$

158 Config -1, Courtyard glazing Trp LoE (e2=e5=.1) Clr 3mm/13mm Air, WWR 36\%, East-Atrium glazing DbI LoE (e2=.1) C 159 Config -1 , Courtyard glazing DbI LoE (e2=.1) Clr 6mm/13mm Air, WWR 77\%, South-Atrium glazing Dble clr, $6 \mathrm{~mm} / 13 \mathrm{~m}$ 160 Config -2, Courtyard glazing Dbl clr $6 \mathrm{~mm} / 13 \mathrm{~mm}$ air, WWR 36\%, West-Atrium glazing Dble clr, $6 \mathrm{~mm} / 13 \mathrm{~mm}$ air

161 Config -2, Courtyard glazing Trp LoE (e2=e5=.1) Clr 3mm/13mm Air, WWR 23\%, South-Atrium glazing Dble clr, 6mm/

162 Config -2 , Courtyard glazing DbI LoE (e2=.1) Clr 6mm/13mm Air, WWR 36\%, South-Atrium glazing Dble clr, $6 \mathrm{~mm} / 13 \mathrm{~m}$

163 Config -2, Courtyard glazing Trp LoE (e2=e5=.1) Clr 3mm/13mm Air, WWR 36\%, South-Atrium glazing Dble clr, 6mm/

164 Config -1 , Courtyard glazing Trp LoE (e2=e5=.1) Clr 3mm/13mm Air, WWR 36\%, North-Atrium glazing Dbl LoE (e2=.1)

165 Config -1 , Courtyard glazing DbI LoE (e2=.1) Clr 6mm/13mm Air, WWR 36\%, East-Atrium glazing Dbl LoE (e2=.1) Clr 6

166 Config -2, Courtyard glazing Dbl clr $6 \mathrm{~mm} / 13 \mathrm{~mm}$ air, WWR 23\%, West-Atrium glazing Dbl LoE (e2=.1) Clr $6 \mathrm{~mm} / 13 \mathrm{~mm}$

167 Config -2, Courtyard glazing Trp Clr 3mm/13mm Air, WWR 23\%, South-Atrium glazing Dbl LoE (e2=.1) Clr $6 \mathrm{~mm} / 13 \mathrm{mr}$

168 Config -2, Courtyard glazing DbI LoE (e2=.1) Clr 6mm/13mm Air, WWR 23\%, South-Atrium glazing DbI LoE (e2=.1) Clr

169 Config -2 , Courtyard glazing Trp Clr 3mm/13mm Air, WWR 36\%, South-Atrium glazing DbI LoE (e2=.1) Clr $6 \mathrm{~mm} / 13 \mathrm{mr}$ 170 Config -2, Courtyard glazing Trp Clr 3mm/13mm Air, WWR 23\%, North-Atrium glazing Dble clr, $6 \mathrm{~mm} / 13 \mathrm{~mm}$ air

171 Config -2 , Courtyard glazing Trp LoE (e2=e5=.1) Clr 3mm/13mm Air, WWR 23\%, East-Atrium glazing Dble clr, 6mm/13

172 Config -2, Courtyard glazing Dbl clr $6 \mathrm{~mm} / 13 \mathrm{~mm}$ air, WWR 23\%, East-Atrium glazing Dbl LoE (e2=.1) Clr $6 \mathrm{~mm} / 13 \mathrm{~mm}$

173 Config -2, Courtyard glazing Trp LoE (e2=e5=.1) Clr 3mm/13mm Air, WWR 36\%, West-Atrium glazing DbI LoE (e2=.1)

174 Config -2 , Courtyard glazing Trp LoE (e2=e5=.1) Clr 3mm/13mm Air, WWR 36\%, South-Atrium glazing Dbl LoE (e2=.1)

175 Config -1, Courtyard glazing Dbl clr $6 \mathrm{~mm} / 13 \mathrm{~mm}$ air, WWR $57 \%$, South-Atrium glazing Dble clr, $6 \mathrm{~mm} / 13 \mathrm{~mm}$ air

176 Config -2, Courtyard glazing DbI LoE (e2=.1) Clr 6mm/13mm Air, WWR 23\%, West-Atrium glazing Dble clr, $6 \mathrm{~mm} / 13 \mathrm{~m}$

177 Config -2, Courtyard glazing Dbl clr $6 \mathrm{~mm} / 13 \mathrm{~mm}$ air, WWR 23\%, North-Atrium glazing Dble clr, $6 \mathrm{~mm} / 13 \mathrm{~mm}$ air

178 Config -1 , Courtyard glazing Dbl LoE (e2=.1) Clr 6mm/13mm Air, WWR 57\%, South-Atrium glazing Dble clr, 6mm/13m

179 Config -2, Courtyard glazing Trp Clr 3mm/13mm Air, WWR 36\%, South-Atrium glazing Dble clr, $6 \mathrm{~mm} / 13 \mathrm{~mm}$ air

180 Config -1, Courtyard glazing DbI LoE (e2=.1) Clr 6mm/13mm Air, WWR 36\%, North-Atrium glazing Dbl LoE (e2=.1) Clr

181 Config -2 , Courtyard glazing DbI LoE (e2=.1) Clr 6mm/13mm Air, WWR 23\%, East-Atrium glazing Dble clr, $6 \mathrm{~mm} / 13 \mathrm{mn}$

182 Config -2, Courtyard glazing Dbl clr $6 \mathrm{~mm} / 13 \mathrm{~mm}$ air, WWR 36\%, East-Atrium glazing DbI LoE (e2=.1) Clr $6 \mathrm{~mm} / 13 \mathrm{~mm} \mathrm{~A}$ 183 Config -1, Courtyard glazing Trp Clr 3mm/13mm Air, WWR 36\%, North-Atrium glazing DbI LoE (e2=.1) Clr 6mm/13mr 184 Config -2, Courtyard glazing Trp Clr 3mm/13mm Air, WWR 23\%, West-Atrium glazing Dble clr, $6 \mathrm{~mm} / 13 \mathrm{~mm}$ air 185 Config -1 , Courtyard glazing Trp Clr 3mm/13mm Air, WWR 36\%, East-Atrium glazing DbI LoE (e2=.1) Clr $6 \mathrm{~mm} / 13 \mathrm{~mm}$ 186 Config -1 , Courtyard glazing Trp LoE (e2=e5=.1) Clr 3mm/13mm Air, WWR 20\%, North-Atrium glazing DbI LoE (e2=.1) 187 Config -2, Courtyard glazing Trp Clr 3mm/13mm Air, WWR 23\%, East-Atrium glazing Dble clr, $6 \mathrm{~mm} / 13 \mathrm{~mm}$ air

188 Config -2, Courtyard glazing Dbl clr $6 \mathrm{~mm} / 13 \mathrm{~mm}$ air, WWR 23\%, East-Atrium glazing Dble clr, 6mm/13mm air 189 Config -1, Courtyard glazing DbI LoE (e2=.1) Clr 6mm/13mm Air, WWR 20\%, North-Atrium glazing Dbl LoE (e2=.1) Clr 190 Config -2, Courtyard glazing Dbl clr $6 \mathrm{~mm} / 13 \mathrm{~mm}$ air, WWR 36\%, South-Atrium glazing Dble clr, $6 \mathrm{~mm} / 13 \mathrm{~mm}$ air 191 Config -1, Courtyard glazing Trp Clr 3mm/13mm Air, WWR 20\%, North-Atrium glazing Dbl LoE (e2=.1) Clr $6 \mathrm{~mm} / 13 \mathrm{mr}$ 192 Config -1, Courtyard glazing Trp Clr 3mm/13mm Air, WWR 77\%, South-Atrium glazing Dble clr, $6 \mathrm{~mm} / 13 \mathrm{~mm}$ air 193 Config -1 , Courtyard glazing Trp LoE (e2=e5=.1) Clr 3mm/13mm Air, WWR 36\%, East-Atrium glazing Dble clr, 6mm/13 194 Config -1, Courtyard glazing Dbl clr 6mm/13mm air, WWR 36\%, East-Atrium glazing Dbl LoE (e2=.1) Clr $6 \mathrm{~mm} / 13 \mathrm{~mm}$ 195 Config -1, Courtyard glazing Dbl clr $6 \mathrm{~mm} / 13 \mathrm{~mm}$ air, WWR 36\%, North-Atrium glazing DbI LoE (e2=.1) Clr $6 \mathrm{~mm} / 13 \mathrm{~mm}$ 196 Config -2, Courtyard glazing Dbl clr $6 \mathrm{~mm} / 13 \mathrm{~mm}$ air, WWR 23\%, West-Atrium glazing Dble clr, $6 \mathrm{~mm} / 13 \mathrm{~mm}$ air 197 Config -2 , Courtyard glazing DbI LoE (e2=.1) Clr 6mm/13mm Air, WWR 23\%, South-Atrium glazing Dble clr, $6 \mathrm{~mm} / 13 \mathrm{~m}$ 198 Config -2, Courtyard glazing Trp Clr 3mm/13mm Air, WWR 23\%, South-Atrium glazing Dble clr, $6 \mathrm{~mm} / 13 \mathrm{~mm}$ air 199 Config -2, Courtyard glazing Dbl clr 6mm/13mm air, WWR 36\%, South-Atrium glazing Dbl LoE (e2=.1) Clr $6 \mathrm{~mm} / 13 \mathrm{~mm}$ 200 Config -2, Courtyard glazing Dbl clr $6 \mathrm{~mm} / 13 \mathrm{~mm}$ air, WWR 23\%, South-Atrium glazing Dbl LoE (e2=.1) Clr $6 \mathrm{~mm} / 13 \mathrm{~mm}$ 201 Config -1 , Courtyard glazing Trp LoE (e2=e5=.1) Clr 3mm/13mm Air, WWR 57\%, South-Atrium glazing Dble clr, 6mm/ 202 Config -1, Courtyard glazing Trp LoE (e2=e5=.1) Clr 3mm/13mm Air, WWR 77\%, South-Atrium glazing Dble clr, $6 \mathrm{~mm} /$ 203 Config -1, Courtyard glazing Trp LoE (e2=e5=.1) Clr 3mm/13mm Air, WWR 36\%, North-Atrium glazing Dble clr, 6mm/ 204 Config -1, Courtyard glazing Trp Clr 3mm/13mm Air, WWR 57\%, South-Atrium glazing Dble clr, $6 \mathrm{~mm} / 13 \mathrm{~mm}$ air 205 Config -1, Courtyard glazing Trp LoE (e2=e5=.1) Clr 3mm/13mm Air, WWR 20\%, West-Atrium glazing DbI LoE (e2=.1) 206 Config -1 , Courtyard glazing Trp LoE (e2=e5=.1) Clr 3mm/13mm Air, WWR 20\%, East-Atrium glazing DbI LoE (e2=.1) C 207 Config -2, Courtyard glazing Dbl clr $6 \mathrm{~mm} / 13 \mathrm{~mm}$ air, WWR 23\%, South-Atrium glazing Dble clr, $6 \mathrm{~mm} / 13 \mathrm{~mm}$ air 208 Config -1 , Courtyard glazing Dbl LoE (e2=.1) Clr 6mm/13mm Air, WWR 20\%, West-Atrium glazing Dbl LoE (e2=.1) Clr 209 Config -1 , Courtyard glazing Trp LoE (e2=e5=.1) Clr 3mm/13mm Air, WWR 20\%, South-Atrium glazing Dbl LoE (e2=.1) 210 Config -1 , Courtyard glazing DbI LoE (e2=.1) Clr 6mm/13mm Air, WWR 36\%, North-Atrium glazing Dble clr, $6 \mathrm{~mm} / 13 \mathrm{~m}$

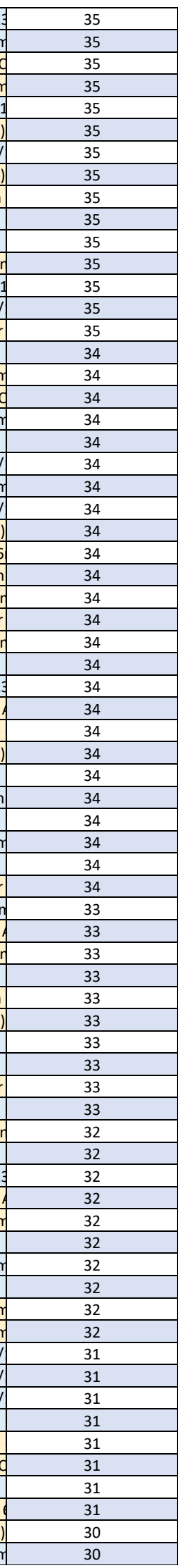




\begin{tabular}{|c|c|c|}
\hline 211 & Config -1, Courtyard glazing DbI LoE (e2=.1) Clr $6 \mathrm{~mm} / 13 \mathrm{~mm}$ Air, WWR 20\%, East-Atrium glazing DbI LoE (e2=.1) Clr 6 & 30 \\
\hline 212 & Config -1 , Courtyard glazing DbI LoE (e2=.1) Clr 6mm/13mm Air, WWR 20\%, South-Atrium glazing DbI LoE (e2=.1) Clr & 30 \\
\hline 213 & Config -1, Courtyard glazing Trp LoE (e2=e5=.1) Clr 3mm/13mm Air, WWR 20\%, North-Atrium glazing Dble clr, $6 \mathrm{~mm} /$ & 30 \\
\hline 214 & Config -1 , Courtyard glazing Dbl clr $6 \mathrm{~mm} / 13 \mathrm{~mm}$ air, WWR 20\%, North-Atrium glazing DbI LoE (e2=.1) Clr $6 \mathrm{~mm} / 13 \mathrm{~mm}$ & 30 \\
\hline 215 & Config -1 , Courtyard glazing Trp LoE (e2=e5=.1) Clr 3mm/13mm Air, WWR 36\%, West-Atrium glazing Dbl LoE (e2=.1) & 30 \\
\hline 216 & Config -1 , Courtyard glazing DbI LoE (e2=.1) Clr 6mm/13mm Air, WWR 36\%, East-Atrium glazing Dble clr, $6 \mathrm{~mm} / 13 \mathrm{mn}$ & 30 \\
\hline 217 & Config -1 , Courtyard glazing Dbl clr $6 \mathrm{~mm} / 13 \mathrm{~mm}$ air, WWR 20\%, East-Atrium glazing DbI LoE (e2=.1) Clr $6 \mathrm{~mm} / 13 \mathrm{~mm}$ & 30 \\
\hline 218 & Config -1, Courtyard glazing Dbl clr $6 \mathrm{~mm} / 13 \mathrm{~mm}$ air, WWR 20\%, West-Atrium glazing Dbl LoE (e2=.1) Clr $6 \mathrm{~mm} / 13 \mathrm{~mm}$ & 30 \\
\hline 219 & Config -1 , Courtyard glazing DbI LoE (e2=.1) Clr 6mm/13mm Air, WWR 36\%, West-Atrium glazing DbI LoE (e2=.1) Clr & 29 \\
\hline 220 & Config -1, Courtyard glazing Dbl clr 6mm/13mm air, WWR 36\%, East-Atrium glazing Dble clr, $6 \mathrm{~mm} / 13 \mathrm{~mm}$ air & 29 \\
\hline 221 & Config -1, Courtyard glazing Dbl clr $6 \mathrm{~mm} / 13 \mathrm{~mm}$ air, WWR 36\%, North-Atrium glazing Dble clr, $6 \mathrm{~mm} / 13 \mathrm{~mm}$ air & 29 \\
\hline 222 & Config -1 , Courtyard glazing DbI LoE (e2=.1) Clr 6mm/13mm Air, WWR 20\%, North-Atrium glazing Dble clr, $6 \mathrm{~mm} / 13 \mathrm{~m}$ & 29 \\
\hline 223 & Config -1, Courtyard glazing Trp Clr 3mm/13mm Air, WWR 36\%, West-Atrium glazing DbI LoE (e2=.1) Clr $6 \mathrm{~mm} / 13 \mathrm{~mm}$ & 28 \\
\hline 224 & Config -1 , Courtyard glazing Trp LoE (e2=e5=.1) Clr 3mm/13mm Air, WWR 20\%, East-Atrium glazing Dble clr, $6 \mathrm{~mm} / 1$ = & 28 \\
\hline 225 & Config -1 , Courtyard glazing Trp LoE (e2=e5=.1) Clr 3mm/13mm Air, WWR 36\%, South-Atrium glazing DbI LoE (e2=.1) & 28 \\
\hline 226 & Config -1, Courtyard glazing Trp LoE (e2=e5=.1) Clr 3mm/13mm Air, WWR 36\%, West-Atrium glazing Dble clr, $6 \mathrm{~mm} / 1$ & 27 \\
\hline 227 & Config -1, Courtyard glazing Dbl clr 6mm/13mm air, WWR 20\%, North-Atrium glazing Dble clr, $6 \mathrm{~mm} / 13 \mathrm{~mm}$ air & 27 \\
\hline 228 & Config -1, Courtyard glazing Trp LoE (e2=e5=.1) Clr 3mm/13mm Air, WWR 20\%, West-Atrium glazing Dble clr, $6 \mathrm{~mm} / 1$ & 27 \\
\hline 229 & Config -1 , Courtyard glazing DbI LoE (e2=.1) Clr $6 \mathrm{~mm} / 13 \mathrm{~mm}$ Air, WWR $20 \%$, West-Atrium glazing Dble clr, $6 \mathrm{~mm} / 13 \mathrm{~m}$ & 27 \\
\hline 230 & Config -1 , Courtyard glazing DbI LoE (e2=.1) Clr $6 \mathrm{~mm} / 13 \mathrm{~mm}$ Air, WWR $20 \%$, East-Atrium glazing Dble clr, $6 \mathrm{~mm} / 13 \mathrm{mn}$ & 27 \\
\hline 231 & Config -1 , Courtyard glazing Trp Clr 3mm/13mm Air, WWR 36\%, South-Atrium glazing DbI LoE (e2=.1) Clr $6 \mathrm{~mm} / 13 \mathrm{mr}$ & 27 \\
\hline 232 & Config -1 , Courtyard glazing Dbl clr $6 \mathrm{~mm} / 13 \mathrm{~mm}$ air, WWR 20\%, South-Atrium glazing DbI LoE (e2=.1) Clr $6 \mathrm{~mm} / 13 \mathrm{~mm}$ & 27 \\
\hline 233 & Config -1 , Courtyard glazing Dbl clr $6 \mathrm{~mm} / 13 \mathrm{~mm}$ air, WWR 36\%, West-Atrium glazing DbI LoE (e2=.1) Clr $6 \mathrm{~mm} / 13 \mathrm{~mm}$ & 27 \\
\hline 234 & Config -1, Courtyard glazing Trp Clr 3mm/13mm Air, WWR 20\%, North-Atrium glazing Dble clr, 6mm/13mm air & 26 \\
\hline 235 & Config -1 , Courtyard glazing Trp LoE (e2=e5=.1) Clr 3mm/13mm Air, WWR 20\%, South-Atrium glazing Dble clr, $6 \mathrm{~mm} /$ & 26 \\
\hline 236 & Config -1, Courtyard glazing DbI LoE (e2=.1) Clr 6mm/13mm Air, WWR 36\%, South-Atrium glazing Dbl LoE (e2=.1) Clr & 26 \\
\hline 237 & Config -1, Courtyard glazing Dbl clr 6mm/13mm air, WWR 20\%, East-Atrium glazing Dble clr, 6mm/13mm air & 26 \\
\hline 238 & Config -1, Courtyard glazing Dbl clr 6mm/13mm air, WWR 20\%, West-Atrium glazing Dble clr, 6mm/13mm air & 26 \\
\hline 239 & Config -1 , Courtyard glazing DbI LoE (e2=.1) Clr 6mm/13mm Air, WWR 36\%, West-Atrium glazing Dble clr, $6 \mathrm{~mm} / 13 \mathrm{~m}$ & 26 \\
\hline 240 & Config -1, Courtyard glazing Trp Clr 3mm/13mm Air, WWR 20\%, West-Atrium glazing Dble clr, 6mm/13mm air & 26 \\
\hline 241 & Config -1 , Courtyard glazing Trp LoE (e2=e5=.1) Clr 3mm/13mm Air, WWR 36\%, South-Atrium glazing Dble clr, $6 \mathrm{~mm} /$ & 25 \\
\hline 242 & Config -1, Courtyard glazing Trp Clr 3mm/13mm Air, WWR 36\%, East-Atrium glazing Dble clr, 6mm/13mm air & 25 \\
\hline 243 & Config -1, Courtyard glazing DbI LoE (e2=.1) Clr $6 \mathrm{~mm} / 13 \mathrm{~mm}$ Air, WWR $20 \%$, South-Atrium glazing Dble clr, $6 \mathrm{~mm} / 13 \mathrm{~m}$ & 25 \\
\hline 244 & Config -1, Courtyard glazing Trp Clr 3mm/13mm Air, WWR 20\%, East-Atrium glazing Dble clr, 6mm/13mm air & 24 \\
\hline 245 & Config -1 , Courtyard glazing DbI LoE (e2=.1) Clr $6 \mathrm{~mm} / 13 \mathrm{~mm}$ Air, WWR $36 \%$, South-Atrium glazing Dble clr, $6 \mathrm{~mm} / 13 \mathrm{~m}$ & 24 \\
\hline 246 & Config -1, Courtyard glazing Dbl clr $6 \mathrm{~mm} / 13 \mathrm{~mm}$ air, WWR 36\%, South-Atrium glazing DbI LoE (e2=.1) Clr $6 \mathrm{~mm} / 13 \mathrm{~mm}$ & 24 \\
\hline 247 & Config -1, Courtyard glazing Trp Clr 3mm/13mm Air, WWR 36\%, North-Atrium glazing Dble clr, 6mm/13mm air & 24 \\
\hline 248 & Config -1, Courtyard glazing Trp Clr 3mm/13mm Air, WWR 20\%, South-Atrium glazing Dble clr, $6 \mathrm{~mm} / 13 \mathrm{~mm}$ air & 23 \\
\hline 249 & Config -1, Courtyard glazing Dbl clr $6 \mathrm{~mm} / 13 \mathrm{~mm}$ air, WWR 20\%, South-Atrium glazing Dble clr, $6 \mathrm{~mm} / 13 \mathrm{~mm}$ air & 23 \\
\hline 250 & Config -1, Courtyard glazing Dbl clr 6mm/13mm air, WWR 36\%, West-Atrium glazing Dble clr, 6mm/13mm air & 23 \\
\hline 251 & Config -1, Courtyard glazing Dbl clr $6 \mathrm{~mm} / 13 \mathrm{~mm}$ air, WWR 36\%, South-Atrium glazing Dble clr, $6 \mathrm{~mm} / 13 \mathrm{~mm}$ air & 21 \\
\hline 252 & Config -1, Courtyard glazing Trp Clr 3mm/13mm Air, WWR 20\%, West-Atrium glazing DbI LoE (e2=.1) Clr $6 \mathrm{~mm} / 13 \mathrm{~mm}$ & 21 \\
\hline 253 & Config -1, Courtyard glazing Trp Clr 3mm/13mm Air, WWR 20\%, East-Atrium glazing Dbl LoE (e2=.1) Clr $6 \mathrm{~mm} / 13 \mathrm{~mm}$ & 21 \\
\hline 254 & Config -1, Courtyard glazing Trp Clr 3mm/13mm Air, WWR 20\%, South-Atrium glazing DbI LoE (e2=.1) Clr $6 \mathrm{~mm} / 13 \mathrm{mr}$ & 19 \\
\hline 255 & Config -1, Courtyard glazing Trp Clr 3mm/13mm Air, WWR 36\%, West-Atrium glazing Dble clr, 6mm/13mm air & 18 \\
\hline 256 & Config -1, Courtyard glazing Trp Clr 3mm/13mm Air, WWR 36\%, South-Atrium glazing Dble clr, $6 \mathrm{~mm} / 13 \mathrm{~mm}$ air & 16 \\
\hline
\end{tabular}

